\title{
Functional characterization and application of 2',5'- branched RNA forming deoxyribozymes using lanthanides as cofactors
}

\author{
PhD Thesis \\ in partial fulfillment of the requirements \\ for the degree "Doctor of Philosophy (Ph.D.)" \\ in the Molecular Biology Program \\ at the Georg August University Göttingen, \\ Faculty of Biology
}

Submitted by

Fatemeh Javadi-Zarnaghi

from Ghom, Iran

Göttingen, August 2013 


\title{
Thesis Committee
}

\author{
Dr. Claudia Höbartner
}

Research Group Nucleic Acid Chemistry,

Max Planck Institute for Biophysical Chemistry,

Göttingen, Germany.

Prof. Dr. Reinhard Lührmann

Department of Cellular Biochemistry,

Max Planck Institute for Biophysical Chemistry,

Göttingen, Germany.

Prof. Dr. Kai Tittmann

Department of Bioanalytics in Albrecht-von-Haller Institute,

Göttingen Center for Molecular Biosciences (GZMB),

Ernst-Caspari-Haus,

Georg-August University Göttingen,

Göttingen, Germany.

Oral examination: October 2013. 


\section{Affidavit}

I hereby declare that my doctoral thesis entitled" Functional characterization and application of 2',5'-branched RNA forming deoxyribozymes using lanthanides as cofactors” has been written independently and with no other sources and aids than quoted. I have indicated the parts which were performed by project collaborators.

Fatemeh Javadi-Zarnaghi

Göttingen, August 2013 


\section{List of Publications}

Javadi-Zarnaghi, F., Höbartner, C., Lanthanide cofactors accelerate DNA-catalyzed synthesis of branched RNA, J. Am. Chem. Soc. 2013, 135, 12839-48.

Wachowius, F., Javadi-Zarnaghi, F., Höbartner, C., Combinatorial mutation interference analysis reveals functional nucleotides required for DNA catalysis, Angew. Chem. Int. Ed. 2010, 49, 8504-8.

Höbartner, C., Büttner, L., Javadi-Zarnaghi, F., Bioorthogonal modifications and cycloaddition reactions for RNA Chemical Biology, in RNA Structure and Folding, (Eds. D. Klostermeier, C. Hammann). DeGruyter 2013 In print (ISBN-10: 3110284596).

Javadi-Zarnaghi, F., Höbartner, C., Functional characterization of a deoxyribozyme that forms biologically related lariat RNAs, manuscript in preparation.

Javadi-Zarnaghi, F.*, Büttner, L.*, Höbartner, C., Terbium assisted synthesis of site-specifically labeled RNAs by DNA catalysts, *these authors contributed equally, manuscript in preparation. 


\section{Table of content}

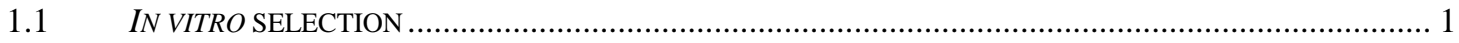

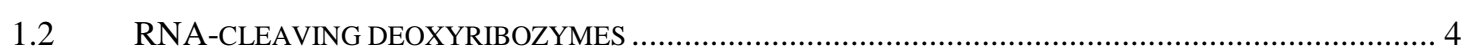

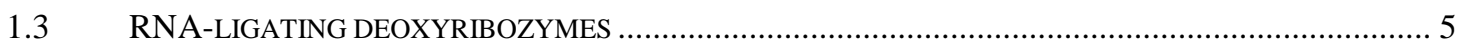

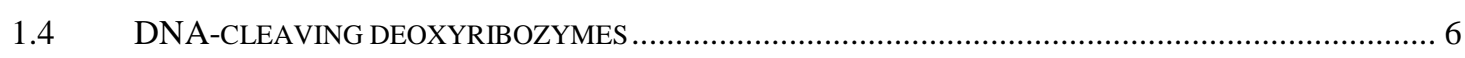

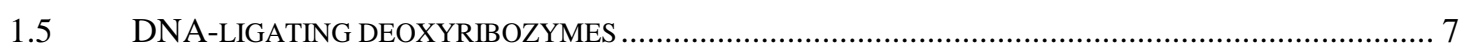

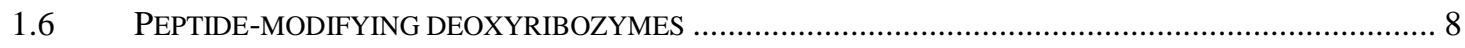

1.7 DEOXYRIBOZYMES WITH SMALL MOLECULES AS SUBSTRATES ................................................... 8

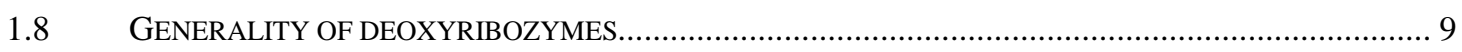

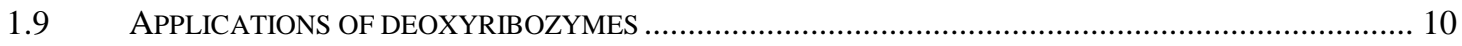

1.10 FUNCTIONAL CHARACTERIZATION OF (DEOXY) RIBOZYMES ......................................................... 13

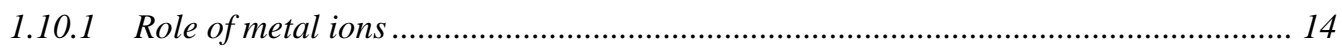

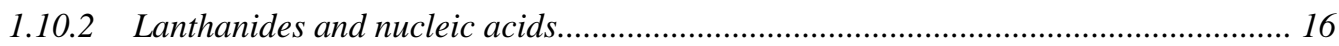

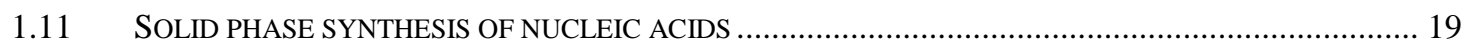

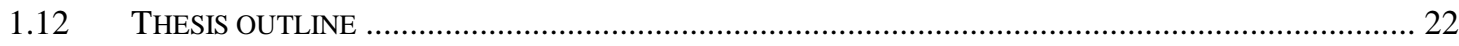

2 FUNCTIONAL CHARACTERIZATION OF DEOXYRIBOZYMES .......................................... 23

2.1 COMBINATORIAL MUTATION INTERFERENCE ANALYSIS (COMA) ............................................. 23

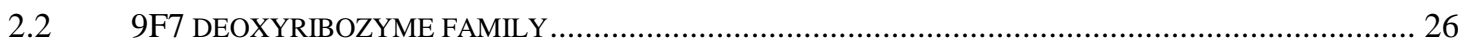

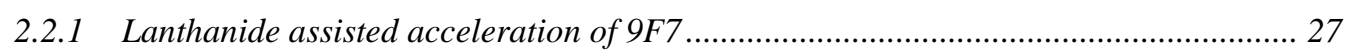

2.2.2 HPLC analysis of the effect of $T^{3+}$ on the reaction components ................................. 31

2.2.3 Combinatorial mutation interference analysis of $9 F 7$................................................... 34

2.2.4 Sensitized luminescence of $\mathrm{Tb}^{3+}$................................................................................ 40

2.2.5 9F7 folding studies by DMS and DNase probing assays........................................... 43

2.2.6 Phosphorothioate rescue experiments ........................................................................... 45

2.2.7 $\quad \mathrm{Tb}^{3+}$ mediated acceleration of other enzymes........................................................... 47

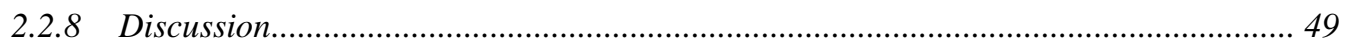

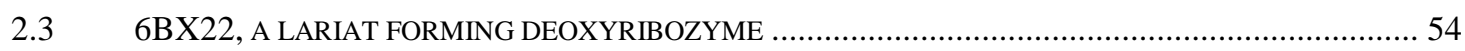

2.3.1 6BX22 substrate requirements................................................................................ 54

2.3.2 Combinatorial mutation interference analysis of $6 \mathrm{BX} 22$.............................................. 56

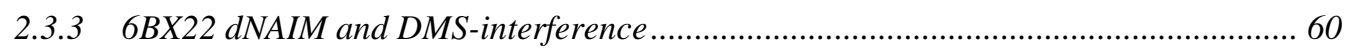

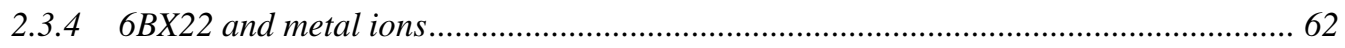

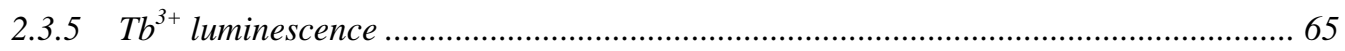

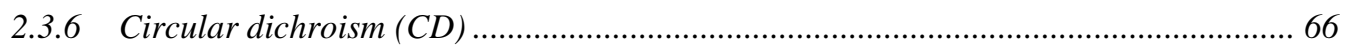

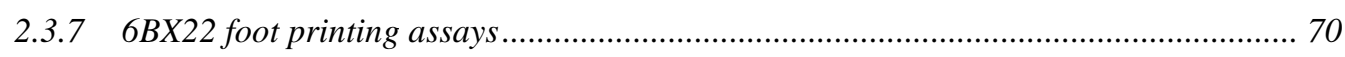

2.3.8 Discussion and outlook.......................................................................................... 73

$3 \quad$ APPLICATION OF LIGATING DEOXYRIBOZYMES ............................................................. 82

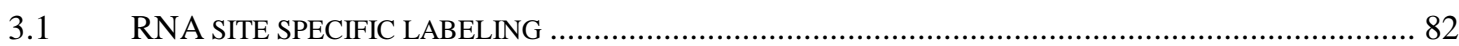


3.1.1 $\mathrm{Tb}^{3+}$ assisted single GTP attachment to RNA scaffold................................................ 84

3.1.2 Reaction scale up for $\mathrm{Tb}^{3+}$ assisted acceleration........................................................ 84

3.1.3 The effect of mutations adjacent to the branch site on GTP attachment ..................... 86

3.1.4 Labeling of RNA with modified NTP in kinetic scale ................................................. 89

3.1.5 Labeling of RNA with modified GTPs in preparative scale ......................................... 90

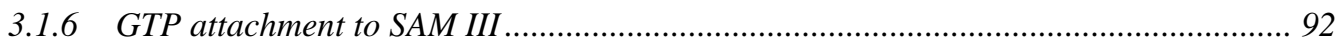

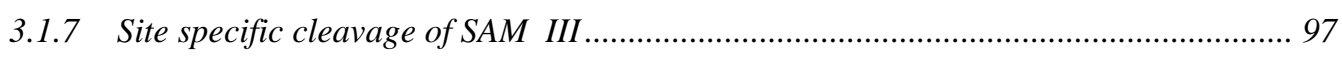

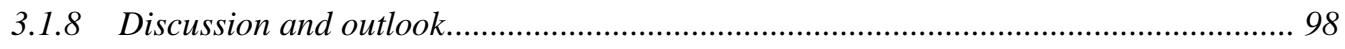

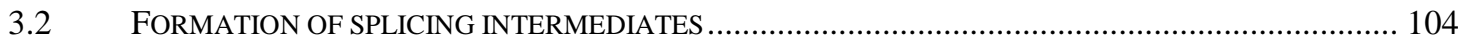

3.2.1 Preparative scale synthesis of 2',5'-branched-RNA.................................................. 105

3.2.2 Binding assays with 2',5'-branched RNAs ............................................................. 105

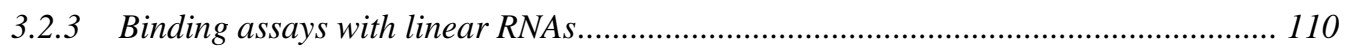

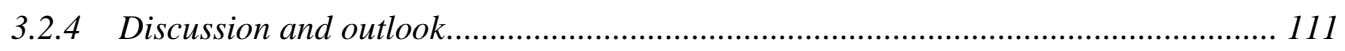

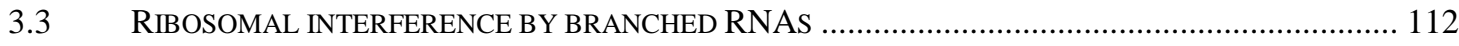

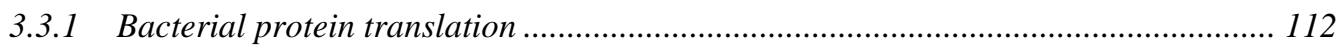

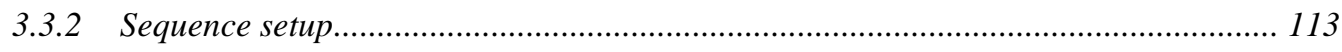

3.3.3 Formation of 70 S initiation complex (70S IC)........................................................ 115

3.3.4 Formation of pre-translocation complex..................................................................... 115

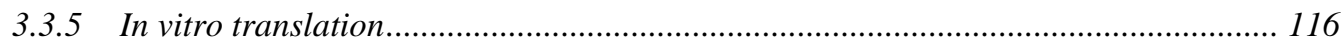

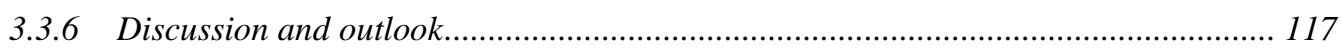

4.1 FUNCTIONAL CHARACTERIZATION OF DEOXYRIBOZYMES

4.1.1 Combinatorial mutation interference analysis revealed necessary nucleotides for catalysis

4.1.2 Nucleotide analogue interference mapping of DNA provided data on functional group demands of catalytic DNAs

4.1.3 Lanthanides have been useful for fundamental studies on deoxyribozymes

4.2 $\mathrm{TB}^{3+}$ MEDIATED ACCELERATION OF RNA-LIGATING DEOXYRIBOZYMES ENHANCED DEOXYRIBOZYMES’ APPLICATION

4.2.1 Site-specific labeling of RNA with modified GTPs was impressively efficient in presence of $\mathrm{Tb}^{3+}$

4.2.2 Preparative synthesis of 2',5'-branched RNA was shown to be useful in molecular biology 124

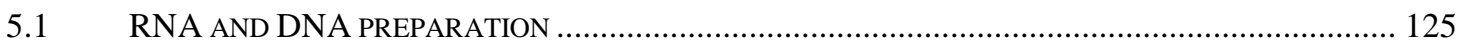

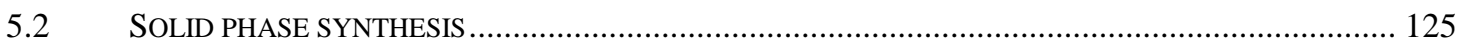

5.2.1 Chemicals and building blocks for solid phase synthesis ...................................... 125

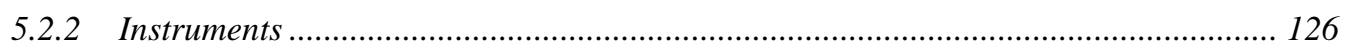

5.2.3 Solutions for solid phase synthesis of oligonucleotides .......................................... 126

5.2.4 Solid phase synthesis and deprotection ............................................................. 126 


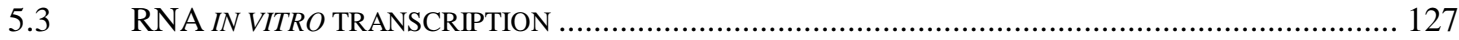

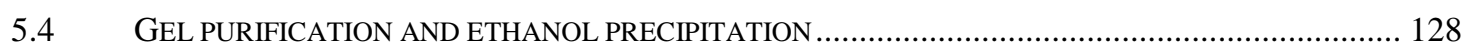

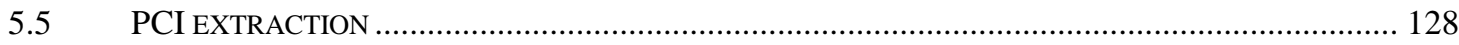

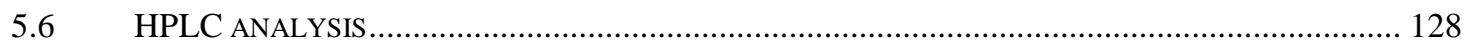

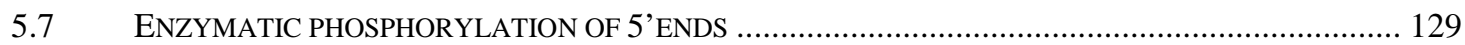

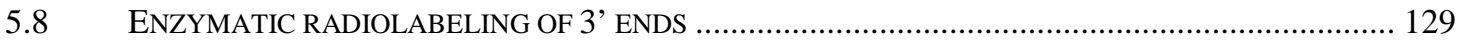

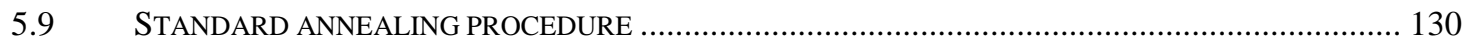

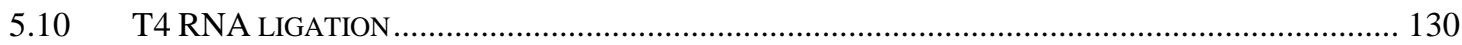

5.11 COMBINATORIAL MUTATION INTERFERENCE ANALYSIS (COMA) ...................................... 130

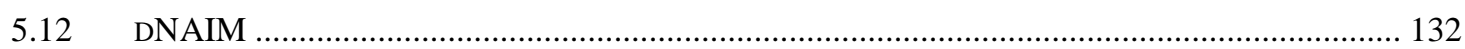

5.13 KINETIC AND PREPARATIVE REACTIONS OF DEOXYRIBOZYMES ........................................... 132

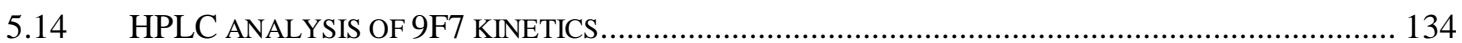

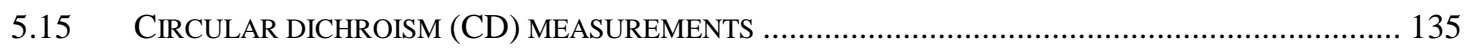

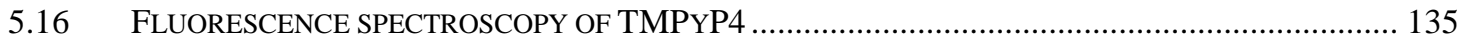

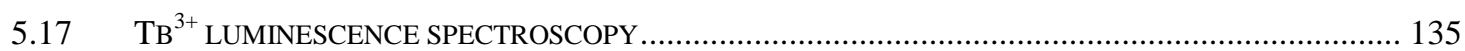

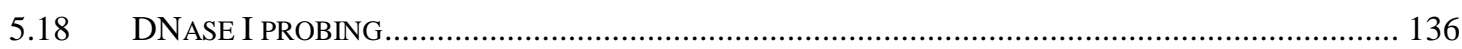

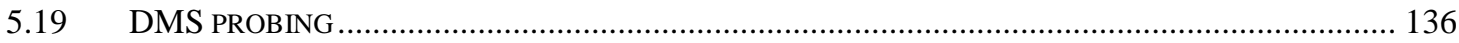

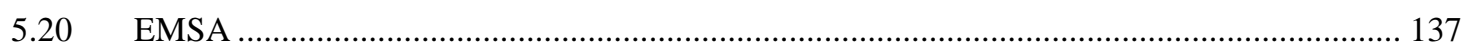

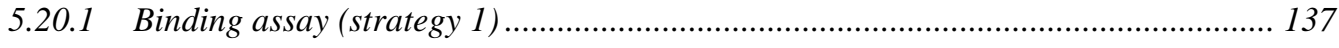

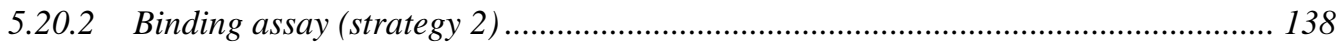

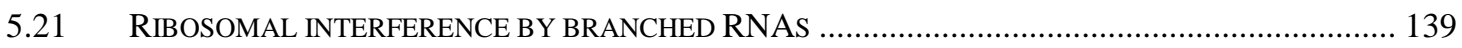

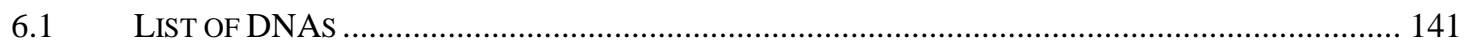

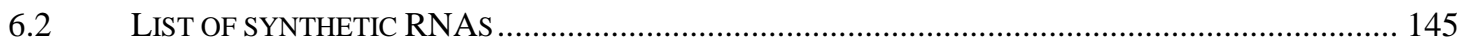

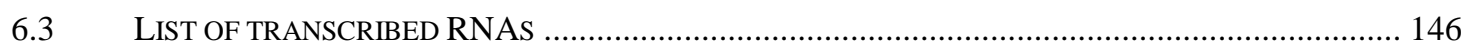

6.4 LIST OF BRANCHED PRODUCTS (PREPARATIVE SCALE) .................................................. 147

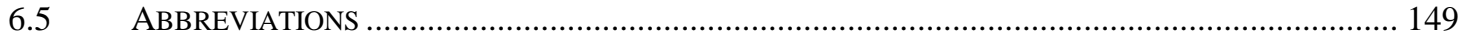

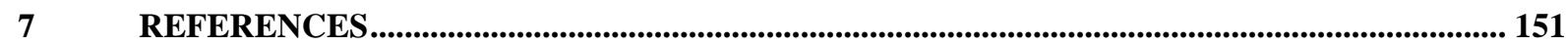

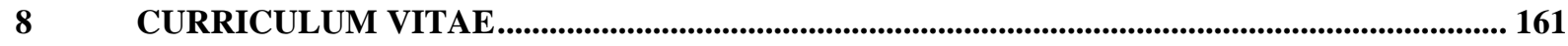




\section{List of Figures}

Fig 1.1-1 General procedure for in vitro selection of RNA ligating deoxyribozymes......................... 3

Fig 1.2-1 Transesterification and hydrolysis reactions of RNA cleaving deoxyribozymes............... 5

Fig 1.3-1 Three helix junction conformation of 7S11 deoxyribozyme family ..................................... 5

Fig 1.5-1 Three step DNA ligation, catalyzed deoxyribozymes. ....................................................... 7

Fig 1.7-1 Schematic of three types of reaction catalyzed by DNA on peptide substrates................... 9

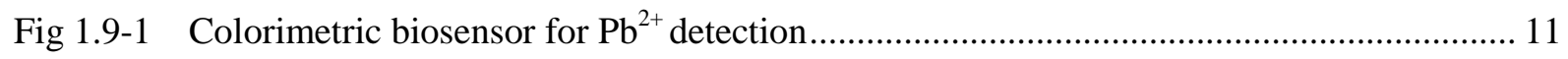

Fig 1.9-2 Deoxyribozymes mediated lariat formation.................................................................. 13

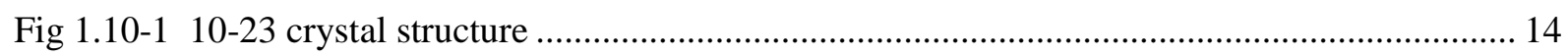

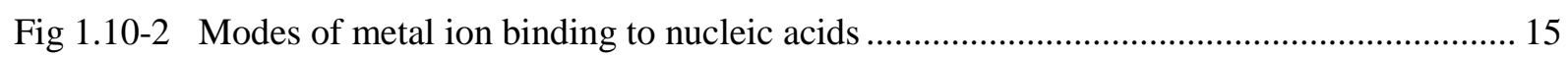

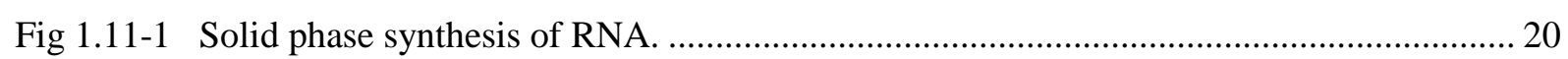

Fig 1.11-2 Activation, coupling and oxidation steps during RNA solid phase synthesis ................... 21

Fig 2.1-1 Combinatorial mutation interference analysis (CoMA) .................................................... 24

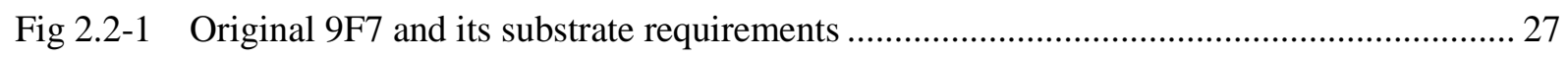

Fig 2.2-2 9 F7 and the effect of $\mathrm{Tb}^{3+}$ on $\mathrm{Mg}^{2+}$ dependent reactions.................................................. 28

Fig 2.2-3 Titration kinetics in presence of $\mathrm{Mg}^{2+}$ for $\mathrm{Eu}^{3+} \mathrm{Gd}^{3+}$ and $\mathrm{Tb}^{3+}$........................................ 28

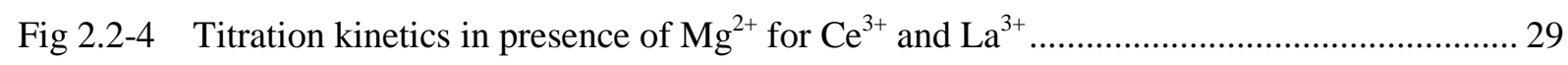

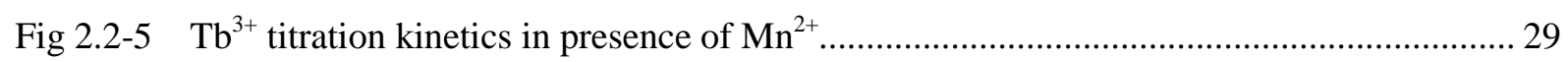

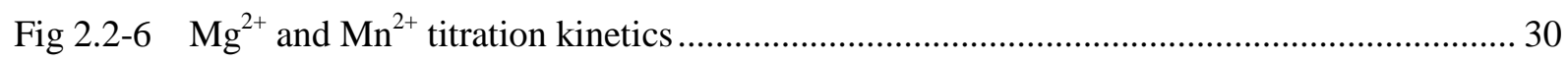

Fig 2.2-7 HPLC analysis of the triphosphorylated donor substrate .................................................. 32

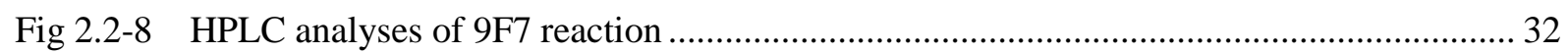

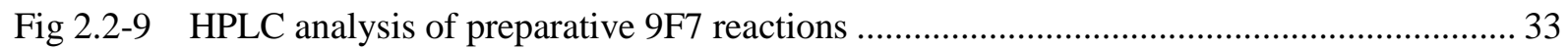

Fig 2.2-10 Stability of branched RNA product of 9F7 .................................................................... 34

Fig 2.2-11 Combinatorial mutation interference analysis of 9F7 ..................................................... 35

Fig 2.2-12 Hydrolysis pattern of 9F7 active mutants at the 5' end of the catalytic core...................... 35

Fig 2.2-13 Interference values for 9F7 mutant libraries at 5 different conditions............................... 36

Fig 2.2-14 Contour graph of interference values of 9F7 combinatorial libraries................................. 37

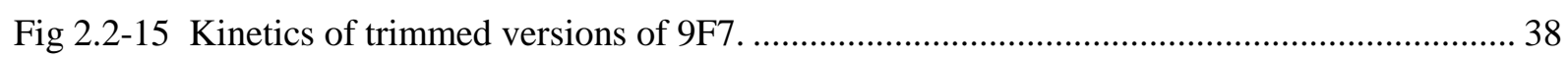

Fig 2.2-16 Interference values of 2‘-OH in the catalytic core of 9F7 ................................................ 39

Fig 2.2-17 Comparison of nucleobases requirements of $9 \mathrm{~F} 7$ for $\mathrm{Mg}^{2+}$ and $\mathrm{Mn}^{2+}$ dependent catalysis. 40

Fig 2.2-18 Sensitized $\mathrm{Tb}^{3+}$ luminescence in competition with $\mathrm{Mn}^{2+}$................................................. 41

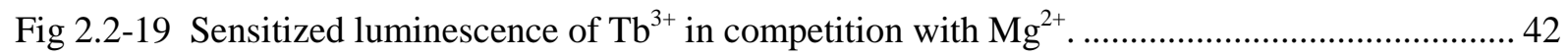

Fig 2.2-20 DMS methylation and piperidine cleavage at guanines.................................................... 43

Fig 2.2-21 DMS and DNase I probing assays of trimmed 9F7 .......................................................... 45

Fig 2.2-22 Phosphorothioate effects and metal ion rescue ............................................................... 47

Fig 2.2-23 $\mathrm{Tb}^{3+}$ mediated acceleration of $\mathrm{Mg}^{2+}$ and $\mathrm{Mn}^{2+}$ dependent reactions of $7 \mathrm{~S} 11$.................... 48 


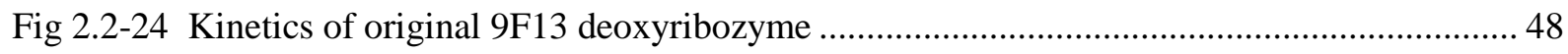

Fig 2.2-25 Combinatorial mutation interference analysis of 9F13 ...................................................... 49

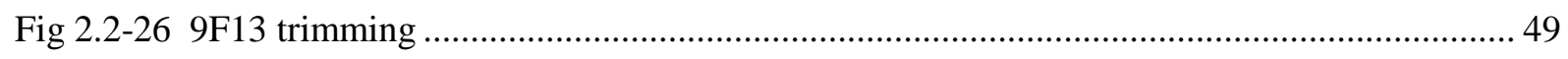

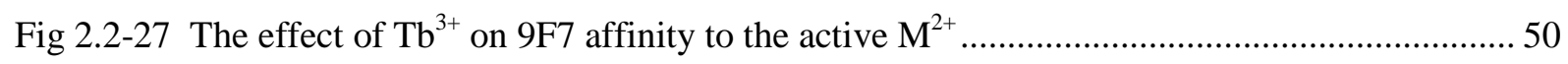

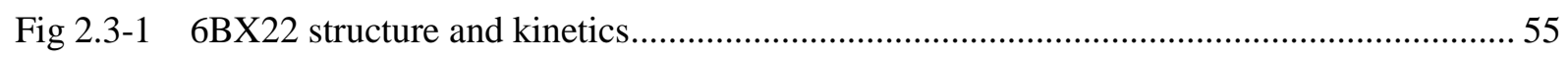

Fig 2.3-2 Kinetic assays for minimization of 6BX22 acceptor RNA............................................... 55

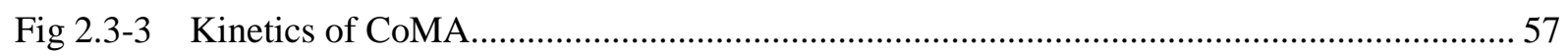

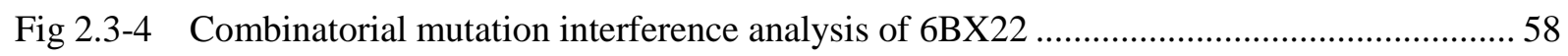

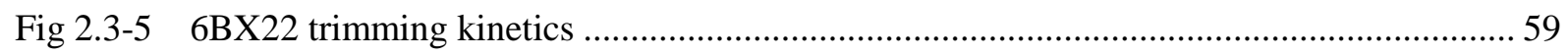

Fig 2.3-6 Point mutant kinetics of trimmed 6BX22 ..................................................................... 59

Fig 2.3-7 Nucleotide analog interference mapping of DNA and DMS-interference of 6BX22......... 61

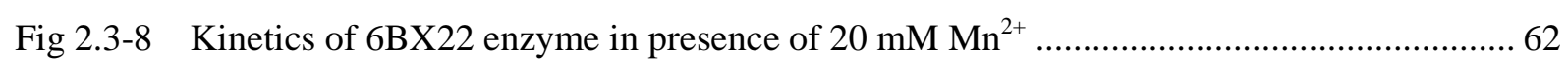

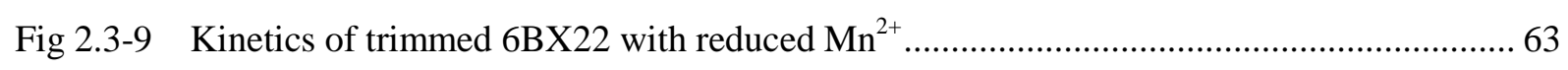

Fig 2.3-10 $\mathrm{Tb}^{3+}$ mediated inhibition of $\mathrm{Mn}^{2+}$ dependent 6BX22 reaction.............................................. 64

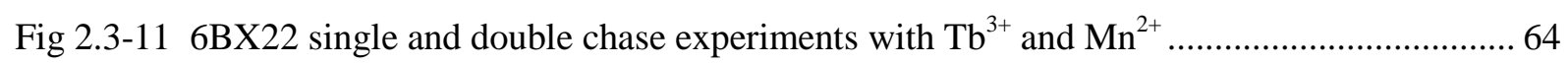

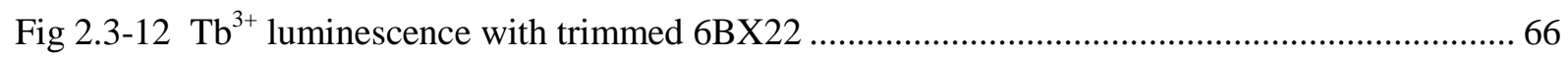

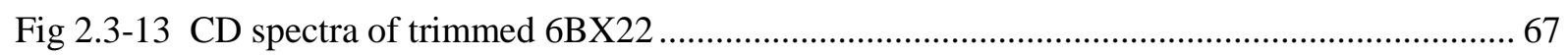

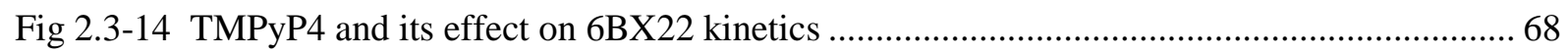

Fig 2.3-15 TMPyP4 fluorescence in presence of 6BX22 DNA and metal ions.................................. 68

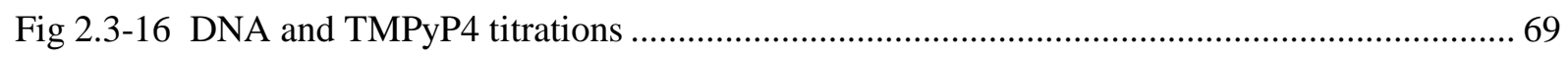

Fig 2.3-17 DMS and DNase I probing of minimized 6BX22 and its inactive point mutant ................ 71

Fig 2.3-18 DMS probing of active 6BX22 and $\mathrm{Tb}^{3+}$ and $\mathrm{Mn}^{2+}$ titrations........................................... 73

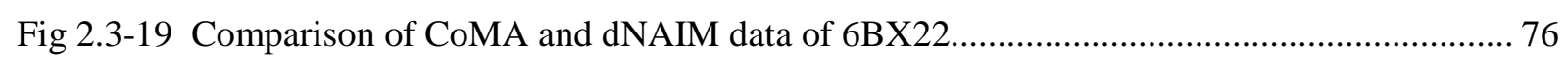

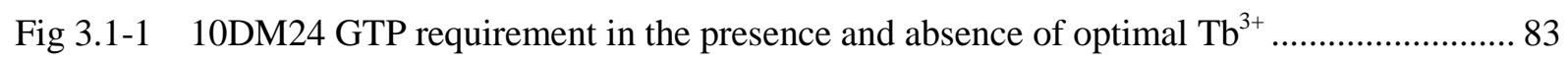

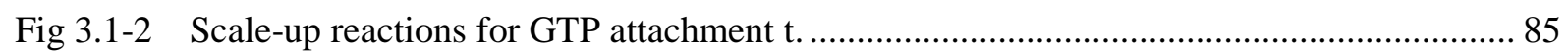

Fig 3.1-3 Kinetic studies for scaffold mutants at the branch site ..................................................... 86

Fig 3.1-4 Ligated fraction for all 16 sequence contexts around branch site...................................... 88

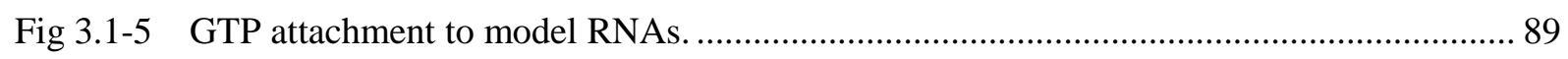

Fig 3.1-6 Attachment of (modified-) NTPs to model RNA ........................................................... 90

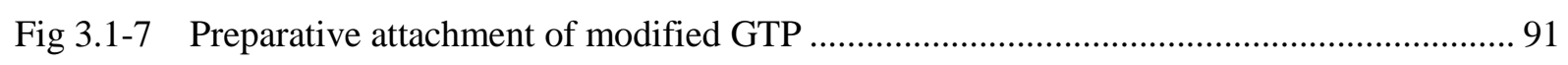

Fig 3.1-8 Confirmation of persistence of the EDA linked labels through 10DM24 reaction............. 92

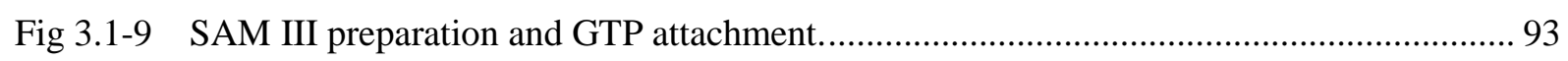

Fig 3.1-10 GTP attachment at reduced ionic strengths .................................................................... 96

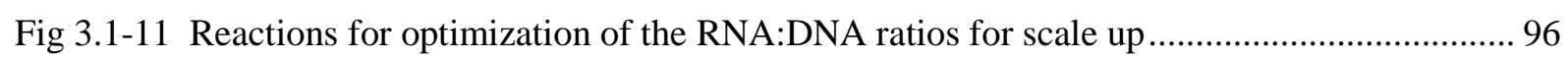

Fig 3.1-12 Cleavage of the dinucleotide junctions AA with E5112 and GU with 10-23 enzyme ....... 98

Fig 3.1-13 Clean-up and analysis strategies for long RNA labeling .............................................. 100

Fig 3.1-14 Schematic plan for labeling, clean up and analysis of SAM III. .................................... 101 
Fig 3.1-15 Schematic plan for labeling, clean up and analysis of U6-snRNA .................................. 102

Fig 3.2-1 Branched RNAs and splicing intermediate complexes................................................... 106

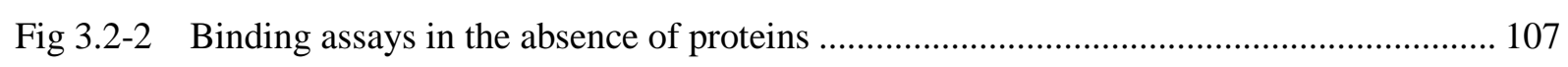

Fig 3.2-3 Titration of Cwc2 and RNase H domain and U6-sh snRNA ........................................ 108

Fig 3.2-4 Comparative complex formation of Cwc2 and RNase H domain and U6-sh snRNA ...... 109

Fig 3.2-5 EMSA in the absence of branched RNA .................................................................. 110

Fig 3.3-1 Hypothetical peptide sequences Hem Kand adaptor RNAs .......................................... 114

Fig 3.3-2 Ligation of adaptor RNAs to the HemK mRNA. ........................................................ 115

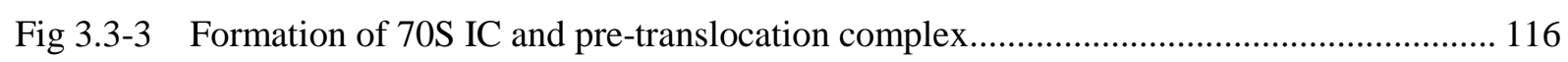

Fig 3.3-4 In vitro translation of branched and non-branched RNAs. .............................................. 117 


\section{List of tables}

Table 2.2-1 Fit values of lanthanide titrations in the presence of $\mathrm{Mg}^{2+}$ or $\mathrm{Mn}^{2+} \ldots \ldots \ldots \ldots \ldots \ldots \ldots \ldots \ldots . . . . . . . . . . . . . . . .30$

Table 2.2-2 Acceleration of 9F7 $\mathrm{Mg}^{2+}$ dependent reactions upon presence of $\mathrm{Tb}^{3+}$......................... 31

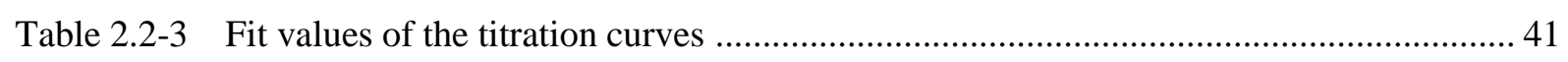

Table 2.2-4 Kinetics of trimmed 9F7 in comparison to G4 mutants............................................... 42

Table 2.3-1 Efficiency of $\mathrm{rN}$ incorporation in a mixture of $\mathrm{rN}: \mathrm{dN}$ phosphoramidites....................... 56

Table 3.1-1 Scale-up conditions for GTP attachment to model RNA............................................... 85

Table 3.1-2 Rate and yield of mutant RNAs for GTP attachment. .................................................. 87

Table 3.1-3 Labeling reactions for full length SAM III in different conditions.................................. 96

Table 5.11-1 Reaction time and conditions for separation step of CoMA ....................................... 131

Table 5.17-1 Preparation of stock solutions for $\mathrm{Tb}^{3+}$ titration........................................................... 136

Table 5.20-1 General preparation of serial dilutions for EMSA ...................................................... 138

Table 5.20-2 Final concentration of RNA and protein components in binding assays ..................... 138 
" الحمد لله الذى هدانا لهذا و ما كنا لنهتدى لولا ان هدانا الله"

$$
\text { تقديم با بوسه بر دستان برمهز بِر و مادرم }
$$

This dissertation would never come to an end without patience and supports of my parents and is dedicated to them. 


\section{Acknowledgments}

I would like to express my sincere gratitude to my supervisor Dr. Claudia Höbartner for her continues intellectual support of my PhD study and research. I will always be indebted to her for introducing me to the field of "DNA catalysts". Her immense knowledge, enthusiasm and energy were "the catalysts" to overcome the activation energy barrier for starting new ideas and projects. Besides her great scientific attitude, I appreciate her patience and her warm and friendly supports and encouragements for academic and non-academic aspects of life.

I thank the thesis committee members Prof. Dr. Reinhard Lührmann and Prof. Dr. Kai Tittmann for patient attendance at thesis committee meetings and reviewing the dissertation.

Special thanks to the PhD program Molecular Biology- International Max Planck Research School for financial supports. I would like to thank Dr. Steffen Burkhardt, Kerstin Grüniger and Ivana Bacakova for the great coordination and supports especially during the first year.

Many thanks to the members of the Research Group Nucleic Acid Chemistry for nice, warm and collaborative environment. Thanks to Dr. Falk Wachowius a former $\mathrm{PhD}$ student of the group for teaching me the initial steps for working with deoxyribozymes and providing precious self-synthesized phosphoramidites for my dNAIM experiments. Thanks to Lea Büttner for her contribution to the comprehensive studies on RNA substrate mutants and additional step by step application of $\mathrm{Tb}^{3+}$ for the RNA labeling project. Thanks to Katarzyna Wawrzyniak-Turek, for scientific and friendly discussions and great coffee times. Thanks to Almudena Ponce-Salvatierra for her positive energies and delicious and unexpected "wish cookies". Thanks to Anne Leonore Ochmann, Sandra Lange, Jan Seikowski, Biswajit Samanta and Johannes Hellwig for friendly and helpful environment in the lab and good language practice starting with the phrase "Hallo Guten Tag!".

Thanks to Prof. Dr. Marina Rodnina and her department for the collaboration on the ribosomal interference project. Many thanks to Riccardo Belardinelli for providing the study material for this project, grateful introduction to the field and excellent discussions.

I consider it an honor to collaborate with the department of Cellular Biochemistry (Professor Dr. Lührmann) and the group of Macromolecular Crystallography (Dr. Vladimir Pena). Thanks to Olexandr Dybkov for providing snRNA plasmid and productive discussions. Thanks to Jana Schmitzova who provided spliceosomal proteins. Thanks to the department of Reinhard Jahn for access to the CD spectrometer. Thanks to the group of Henning Urlaub for measuring mass spectra of synthesized RNA and DNA oligonucleotides. 
I would like to express my gratitude to all my friends with whom I spent happy days. I appreciate their supports and helps during hard times. Being far away from home and family, they never allowed me to feel lonely. I am indebted to Zohreh for being besides me as a supportive sister. I would like to offer my special thanks to Fatemeh, Mahdokht, Atefeh, Tahereh, Farnaz, Asieh, Mahboubeh, Sara, Samane and Azam for special happy moments as well as get togethers full of fun and new life lessons.

\begin{abstract}
$* * * * * *$
My deepest gratitude is to my parents for their blessings and unlimited support through all stages of my life. I would like to thank my Mother for her patience during the years I was away from home for my studies. I am deeply indebted to my Father who always encouraged me to achieve one step further in my academic life. I would like to thank my sisters and my brother for their supports. Zahra, Masoumeh, Reihaneh and Mohammad-Mahdi thank you for making home a warm and nice place for our parents especially when I was not there.
\end{abstract}




\section{Abstract}

Deoxyribozymes or DNA catalysts are synthetic single stranded DNA molecules capable of catalysis for a wide range or reactions. Deoxyribozymes are discovered by in vitro selection and are mostly dependent on bivalent metal ions for their activity. Very little is known about the structure and function of deoxyribozymes. The role of metal ions in the catalytic mechanism of deoxyribozymes is not well understood. The research presented in this thesis describes functional characterization of RNA ligating deoxyribozymes. In particular, the class of deoxyribozymes which form 2',5' branchedRNA has been studied in detail.

To investigate necessary nucleotides and functional groups for DNA catalysis, combinatorial mutation interference analysis and nucleotide analogue interference mapping of DNA have been developed. Additional biochemical and biophysical techniques including DMS interference, kinetic studies in the presence of combinations of monovalent and bivalent metal ions and trivalent lanthanides, phosphorothioate rescue experiments, DMS and DNase footprinting assays and spectroscopic methods (i.e. CD and fluorescence spectroscopy) have been employed to achieve better understanding about the mechanism of DNA catalysis.

Lanthanides and specifically $\mathrm{Tb}^{3+}$ have been shown to be effective accelerators of several 2',5'branched RNA forming deoxyribozymes (e.g. 9F7, 9F13, 7S11 and 10DM24), when present in the appropriate concentrations. For 9F7, $100 \mu \mathrm{M} \mathrm{Tb}^{3+}$ in combination with $7 \mathrm{mM} \mathrm{Mg}^{2+}$ accelerated the enzyme up to 10000 -fold. The acceleratory effect of other lanthanides $\left(\mathrm{La}^{3+}, \mathrm{Ce}^{3+}, \mathrm{Gd}^{3+}, \mathrm{Eu}^{3+}\right.$ and $\mathrm{Yb}^{3+}$ ) was also observed when provided in concentrations ca $100 \mu \mathrm{M}$ in combination with $80 \mathrm{mM}$ $\mathrm{Mg}^{2+}$. The optimal $\mathrm{Tb}^{3+}$ concentration for $\mathrm{Mn}^{2+}$-dependent reactions of 9F7, 9F13 and 7S11 was 10 $\mu \mathrm{M}$. In contrast to the beneficial effect of lanthanides for DNA-catalyzed branching reactions, a deoxyribozyme that synthesizes lariat RNA (6BX22) was inhibited by $\mathrm{Tb}^{3+}$.

The 9F7 deoxyribozyme was studied in most detail. We have shown that presence of optimal concentration of $\mathrm{Tb}^{3+}$ increases the affinity of the enzyme to the active bivalent metal ion. Additional investigations e.g. phosphorothioate rescue experiments, foot printing and $\mathrm{Tb}^{3+}$ binding assays pointed toward activation of the 2'-OH at the branch site. Simultaneous coordination of the active metal ions with the 2'-OH electrophile and oxygen atoms of the nucleophile is conceivable.

The ratio of the metal ions $\left(\mathrm{Tb}^{3+}\right.$ and $\mathrm{M}^{2+}$ ) to nucleic acid components of the reaction was crucial for fast ligation. For 9F7 deoxyribozyme 100-fold excess of $\mathrm{Tb}^{3+}$ to oligonucleotides was required for preparative reactions reaching high yield within a short time. For 10DM24 deoxyribozyme, $\mathrm{Tb}^{3+}$ to DNA ratio was required to be only slightly above 10. 
The results of the fundamental studies on deoxyribozyme mechanism and folding reported in this thesis, expanded practical applications of 2',5' branched-RNA forming deoxyribozymes. Three immediate in vitro applications of branch forming deoxyribozymes have been introduced.

$\mathrm{Tb}^{3+}$ mediated acceleration of 10DM24 was employed for post synthetic RNA labeling. The site specific attachment of a single guanosine to the RNA molecule in presence of $\mathrm{Tb}^{3+}$ was efficient and fast. Compared to the original report on using GTP as substrate for10DM24, $\mathrm{Tb}^{3+}$ accommodated the reaction at more neutral conditions $(\mathrm{pH}$ 7.5). The deoxyribozyme apparent affinity for GTP was significantly increased. The optimum GTP concentration for labeling was decreased from $3 \mathrm{mM}$ to 10 $\mu \mathrm{M}$ in kinetic scale. In preparative scale only 4-fold excess of GTP to RNA substrate was sufficient to reach high yields in less than 1 hour.

$\mathrm{Tb}^{3+}$ mediated acceleration of 10DM24 deoxyribozyme was also employed to ligate adaptor RNAs to an mRNA as scaffold RNA to form a branched mRNA for in vitro translation experiments, in which the ribosome response to the branched mRNA was studied. The results suggested that ribosome is not able to bypass the branch point. The ribosomal interference has potential applications in the field of cellular and molecular biology.

Preparative scale syntheses of branched RNAs with sequence similarities to the yeast actin mRNA have been achieved using 6BX22 deoxyribozyme. Preliminary binding assays have been performed with 2',5' branched RNA products (resembling part of the lariat RNA) and spliceosomal proteins and snRNA fragments. 


\section{Introduction}

Known natural biocatalysts are mostly proteins. However, since 1980s potential abilities of nucleic acids became a notable scientific interest. In 1981, Tomas Cech, reported conservatively on RNA-based catalysis in ciliated protozoan Tetrahymena ${ }^{1}$. In 1984, Sydney Altman and his coworker announced the catalytic activity of RNase P in the absence of any protein component ${ }^{2}$. Since then several natural ribozymes have been discovered which most of them are involved in trans-phosphorylation reactions ${ }^{3}$. Examples include hammerhead ribozyme ${ }^{4}$, hairpin ribozyme ${ }^{5}$ and group II intron ${ }^{6}$. Since discovery of the ribozymes, the catalytic ability of DNA was doubted, however, in vitro selection has been used to discover artificial catalytic DNAs. The first successful discovery of deoxyribozymes was an answer to the fundamental question about the catalytic ability of the DNA in the absence of the 2'-OH functional group.

\subsection{In vitro selection}

Naturally, DNA is responsible for genetic information storage and is double stranded. DNA lacks the properties that proteins use for catalysis such as $\mathrm{p} K_{\mathrm{a}}$ near neutral condition (as in histidines), primary alkyl amines (as in lysines), carboxyl groups (as in aspartates) and sulfhydryl groups (as in cysteines). DNA also lacks the 2'-OH group which is present in ribozymes and is anticipated to be involved in the catalytic mechanism of ribozymes ${ }^{7}$. Despite doubts about possibility of existence of catalytic DNAs, in vitro selections have been performed to discover catalytically active DNA sequences.

In vitro selection was first used to identify artificial RNA catalysts (ribozymes). In 1990, two independent groups reported on strategies to select functional nucleic acids. Systematic evolution of ligands by exponential enrichment (SELEX) was introduced by Tuerk and Gold ${ }^{8}$. They report on a selection procedure to identify specific RNA ligands to bacteriophage T4 DNA polymerase. During the same year, Robertson and Joyce, had reported an in vitro selection strategy to enrich an RNA sequence with DNA cleavage activity within a randomized pool of RNA sequences ${ }^{9}$.

In vitro selection for DNA-catalysts is performed with single stranded DNA libraries. It is anticipated that in a random library of DNA molecules there are sequences which can adapt to a three-dimensional fold that fulfills catalysis. The first in vitro selected deoxyribozyme, was a $\mathrm{Pb}^{2+}$ dependent $\mathrm{RNA}$ cleaving DNA and was discovered in 1994 by Breaker and Joyce ${ }^{10}$. The cleavage reaction obeyed Michaelis-Menten kinetics with a multiple turnover $k_{\text {cat }}$ value of $1 \min ^{-1}$ at $23^{\circ} \mathrm{C}$ and $\mathrm{pH}$ 7.0. The deoxyribozyme discovered by Breaker and Joyce provided a rate enhancement of $10^{5}$-fold over the uncatalyzed reaction. In vitro selection starts with a random DNA library which is prepared by solidphase synthesis. The DNA library contains a random region (usually 40-nucleotide long, $\mathrm{N}_{40}$ ) embedded between two fixed DNA strands that serve as PCR primer binding sites. Permutation of 4 nucleotides at 40 positions forms a sequence space ${ }^{11}$ of about 2 moles $\left(1.2 \times 10^{24}\right)$. The number of molecules by which 
the selection procedure starts is $0.2-1 \mathrm{nmol}$ which covers small portion of the sequence space ( $\sim 1$ out of $\left.10^{10}\right)$. In vitro selection consists of several iterated rounds. The procedure of in vitro selection for discovery of RNA ligating deoxyribozymes is described here (Fig 1.1.-1). The general procedure of in vitro selection for other types of deoxyribozymes is similar with variations at the separation step.

In the first step, DNA library is covalently attached to one RNA substrate. In the next step, by supplying the second RNA substrate, DNA sequences are allowed to catalyze the reaction of interest i.e. ligation of the second RNA to the substrate which has been already covalently bound to the DNA library. Buffer $\mathrm{pH}$, metal ions, temperature and incubation times are adjusted according to the desired stringency. The selection pressure is often increased in later rounds of selection. The next step is separation of active molecules. For RNA ligating deoxyribozymes, separation is trivial and is based on the fact that the molecular weight of the entire RNA-DNA conjugate is larger for the DNA species which actively ligated the two RNAs. Catalytically active DNA sequences can be separated by denaturing polyacrylamide gel electrophoresis (PAGE). The last step of each round is (error-prone) PCR to amplify the active DNAs. Templated polymerase activity (PCR) results in double stranded DNA molecules. To separate the two strands, a DNA overhang is conjugated to the reverse primer with a non-amplifiable linker e.g. polyethylene glycol (PEG) or C3-alkyl linker. The tailed-primer facilitates separation of the active sequence of the PCR product from its complement using denaturing PAGE. The selection is iterated $~ 8-12$ rounds, enriching the population of active DNAs with the desired criteria e.g. high rate or reduced metal ion dependence. Finally, individual deoxyribozyme sequences are cloned, sequenced and characterized $^{11}$.

Here standard procedure was explained for separation of the active molecules based on the size difference and gel retardation. This separation strategy is not always useful. One of the challenges for in vitro selection is to separate only the desired active DNAs but not those sequences which perform an undesired reaction that lead to the same molecular weight. For example historically, attempts to discover deoxyribozymes which catalyze 2'-5' or 3'-5' linear ligation led to discovery of 2',5' branch forming deoxyribozymes. Clever strategies have been designed to overcome such problems. A negative selection pressure was applied after the DNA catalyzed ligation step to eliminate those active DNAs which were catalyzing the undesired reactions. A previously known deoxyribozyme for RNA cleavage at 3'-5' junctions ${ }^{12}$ was used to cleave the ligated RNAs containing the desired 3'-5' linkage. Those RNA-DNA conjugates which were not cleaved by the RNA-cleaving deoxyribozyme were anticipated to have 2',5' linkage and were discarded after a PAGE separation ${ }^{13}$. 


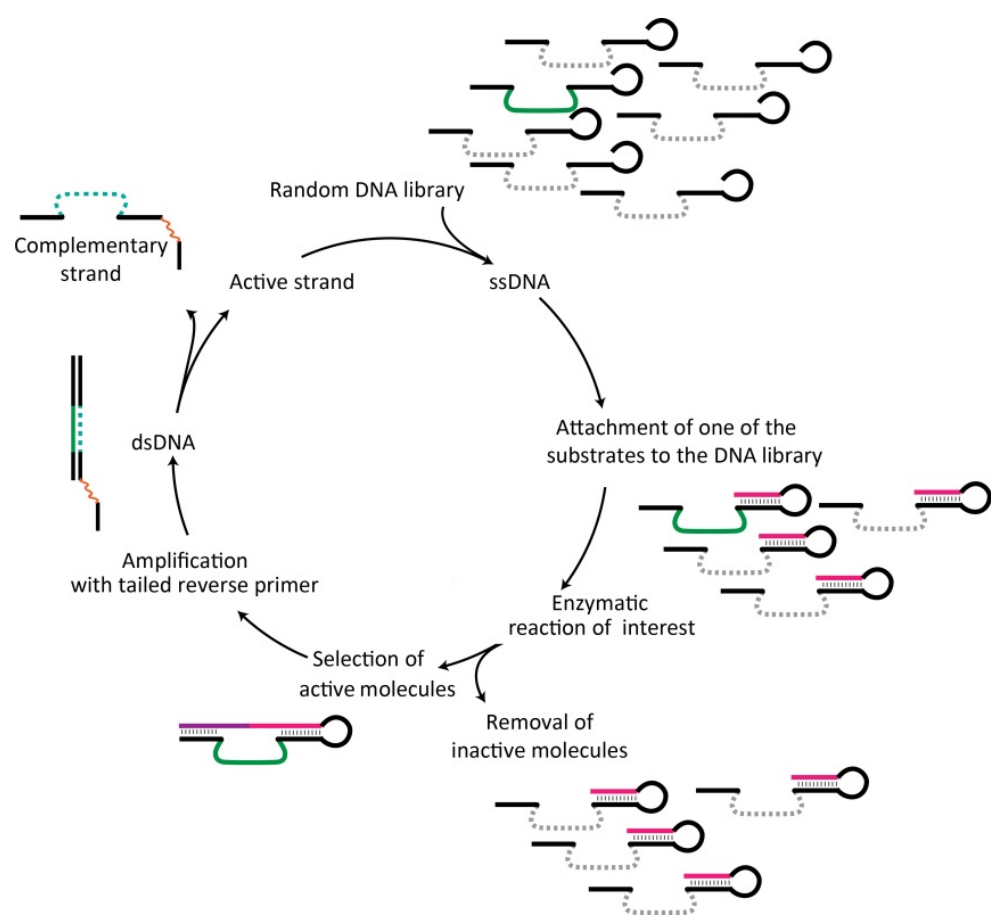

Fig 1.1-1 General procedure for in vitro selection of RNA ligating deoxyribozymes. In vitro selection consists of several rounds of enrichment of the active molecules within a random library. The first round of selection starts with a library of single stranded DNA with random sequences. The length of the random region of each DNA is usually 40 nucleotides. The donor RNA substrate is ligated to the 5' end of the DNA library using T4 RNA ligase. The acceptor RNA substrate and metal ions are provided in the next step to support the enzymatic reaction of interest (here RNA ligation). Active molecules are separated by PAGE and amplified by PCR. For PCR a reverse primer bearing a non-amplifiable region (usually a C3-alkyl linker with a DNA overhang) is used which allows separation of the sense strands (the active sequence) from their complementary strands by denaturing PAGE. Active strands are used for the next rounds of selection.

Employing oligonucleotides as substrates, in vitro selection allowed discovery of deoxyribozymes for catalysis of formation, or cleavage of phosphodiester bonds ${ }^{14}$. However deoxyribozymes are not limited to nucleic acid substrates. Deoxyribozymes catalyze a wide range of reactions ${ }^{15}$. Examples include the catalysis of Diels-Alder reactions, thymine dimer repair and formation of nucleopeptide linkage between amino acid's side chains and RNA molecules. The reactions of deoxyribozymes are not always accommodated with large changes in the molecular weight of the active DNAs. For example in vitro selection for deoxyribozyme capable of dephosphorylation of a substrate is not possible by using standard gel shift at the separation step. The hydrolysis of the phosphate group is not sufficient to cause gel retardation of the DNA-substrate conjugate. In a report by Silverman and coworker on the discovery of phosphatase deoxyribozyme for dephosphorylation of a tyrosine in a peptide chain $^{16}$, a previously selected deoxyribozyme ${ }^{17}$ was used to capture the dephosphorylated peptides-DNA conjugates. The dephosphorylated tyrosines (within the catalytically active DNA-peptide conjugate) were covalently attached to a DNA strand which facilitated their separation by gel shift ${ }^{16}$.

Beneficially deoxyribozymes require only metal ions (usually $\mathrm{Mg}^{2+}, \mathrm{Mn}^{2+}$ or $\mathrm{Zn}^{2+}$ ) as cofactors for their catalytic activity and can easily be isolated and reused for multiple reactions. DNA catalysts can be utilized as tools in molecular biology or as components of biosensors and molecular logic gates ${ }^{18}$. In the 
next few sections several classes of deoxyribozymes are described with a wide range of catalytic abilities on various substrates.

\subsection{RNA-cleaving deoxyribozymes}

10-23 and 8-17 are two well-known RNA cleaving deoxyribozymes ${ }^{12}$. 10-23 has the ability to cleave any purine-pyrimidine junction with robust activity for A-U and G-U sites, and significantly reduced activity for A-C and G-C sites ${ }^{19}$. 8-17 originally was selected for the cleavage at A-G junction. Both 1023 and 8-17 deoxyribozymes have been selected in presence of $\mathrm{MgCl}_{2}$ but addition of $\mathrm{MnCl}_{2}$ improves their reaction rates. There have been several reports to generalize application of RNA cleaving deoxyribozymes for any dinucleotide junctions ${ }^{20,21}$.

A variant of 8-17 deoxyribozyme, 8-17 NG, was selected for DNA substrates with a ribo-junction at the cleavage site. 8-17 NG best catalyzes purine-purine junctions compared to pyrimidine-pyrimidine junctions. ${ }^{20}$ In 2004, Cruz et al. ${ }^{21}$ performed a new in vitro selection with a randomized pool biased to the 8-17 sequence and predicted secondary structure. They also used DNA substrates with only one ribonucleotide at the cleavage junction. The outcome DNA enzymes showed modular similarities to the 8-17. Neither of the enzymes selected by Cruz et al. were able to cleave all the possible junctions. Nevertheless they report efficient cleavage for the enzymes E2112 and E5112 for $\mathrm{NrA}$ and $\mathrm{NrC}$ junctions, E1111 for $\mathrm{NrG}$ and $\mathrm{NrC}$ junctions, E1722 for $\mathrm{NrA}$ and $\mathrm{NrG}$ junctions, E2121 for $\mathrm{NrG}$ junctions when a combination of $\mathrm{Mg}^{2+}$ and $\mathrm{Mn}^{2+}$ ions were provided. In the presence of only $\mathrm{Mg}^{2+}$ the substrate generality of these enzymes was shown to be more limited. One should note that these enzymes were selected with a DNA/RNA chimeric substrate and their activity for RNA substrate was not proved. ${ }^{21}$

Most of the RNA-cleaving deoxyribozymes catalyze 2'-hydroxyl transesterification and form 2',3'cyclic phosphate and 5'-hydroxyl termini. A recent report by Silverman and coworkers introduced a series of new deoxyribozymes for RNA hydrolysis, resulting in 3'-OH and 5'-phosphate groups of the cleaved products $^{22}$. Employing T4 DNA or T4 RNA ligase in the capture step of in vitro selection they positively selected for the desired active sequences. T4 DNA ligase and T4 RNA ligase require 3'-OH and 5'-phosphate termini for ligation reaction. The selection emerged three classes of RNA hydrolyzing enzymes i.e. 9SE, 9SH and 9SK deoxyribozyme families. Figure 1.2-1 depicts the differences of the reaction mechanism and products of transesterification reaction (catalyzed by 10-23, 8-17) and RNA hydrolysis (catalyzed by 9SE, 9SH and 9SK families). 

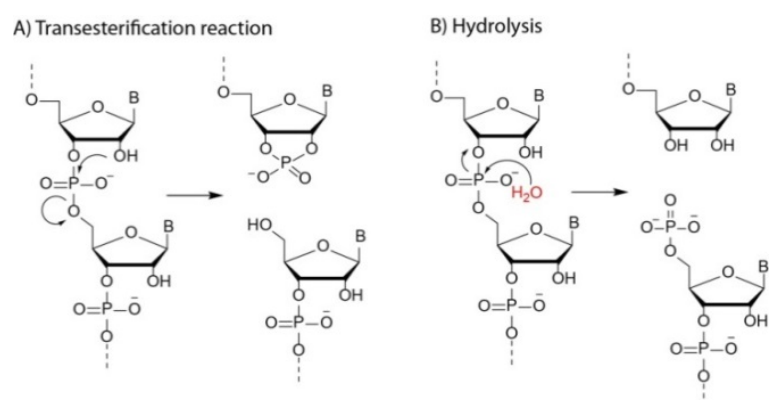

Fig 1.2-1 Transesterification (A) and hydrolysis (B) reactions of RNA cleaving deoxyribozymes. Examples of deoxyribozymes which catalyze transesterification reactions are 10-23 and 8-17. Members of 9SE and 9SH deoxyribozyme family, in the other hand, catalyze cleavage of an RNA substrate by hydrolysis.

\subsection{RNA-ligating deoxyribozymes}

Since 2001, Silverman and coworkers set up several in vitro selections to find RNA ligating deoxyribozymes using 2',3'-cyclic phosphate RNA with 5'-hydroxyl RNA or 3'-hydroxyl RNA with 5'triphosphate RNA. Theoretically, formation of native 3'-5', non-native linear 2'-5' or branched 2',5' phosphodiester bonds are possible with both substrate combinations. However, most of the efforts to discover RNA ligating deoxyribozymes resulted in discovery of 2',5' branch forming deoxyribozymes. Examples include $7 \mathrm{~S} 11^{23}$, 9F7 ${ }^{24,25}, 6 \mathrm{BX} 22^{26}, 6 \mathrm{CE} 8^{27}$ and 10DM24 $4^{28} .7 \mathrm{~S} 11$ family deoxyribozymes adapt a specific structure by forming a three-helix junction (3HJ) that brings the nucleophile i.e. 2'-OH of the branch site in the vicinity to the electrophile i.e. $\alpha$-phosphate of the donor RNA (Fig 1.3-1). The three helix junction fold of 7S11 was taken as a structural model for rational design of new deoxyribozymes.

Most of the discovered 2',5'-branch forming deoxyribozymes prefer adenosine as the branch nucleotide $^{28}$. However, there are exceptions to this general "rule". For example 6CE8 accepts all nucleotides as branch site with preferences of $U>C=A>G^{27}$. $6 B X 22$ accepts $A>U>C$ as branch site. $G$ was accepted by 6BX22 but the rate and yield of the reaction were reduced ${ }^{26}$. Interestingly, members of 9F7 family select different nucleotide as branch site while using the same RNA substrate as the acceptor RNA. The RNA substrate used for in vitro selection of these enzymes contained a $\underline{\text { UAUN }}$ overhang (N stands for any nucleotide). 9F13 and 9F18 chose the first $\underline{\mathbf{U}}$ overhang as branch site while 9F7 and 9F21 take the 2'-OH of the adjacent $\underline{\mathbf{A}}$ as their nucleophile ${ }^{24}$.

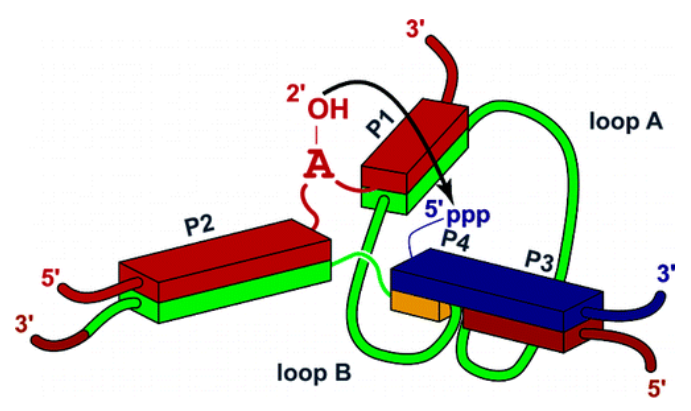

Fig 1.3-1 Three helix junction (3HJ) formed by 7S11 deoxyribozyme family. The sequences of acceptor RNA upstream and downstream of the branch site are involved in formation of $\mathrm{P} 1$ and $\mathrm{P} 2$ paired regions. The length of the $P 1$ is 5 nucleotides long. Additionally first four nucleotide of the donor RNA are involved in formation of the P4 paired region with four nucleotides of the loop $B$ in the catalytic core. P4 is extended by base pairing of the donor RNA with the right binding arm of the DNA. Figure is adapted from ${ }^{29}$. 
On the way to discover 3'-5' ligating deoxyribozymes with high yield and acceptable reaction rate which can be used as general ligase (i.e. capable for $\mathrm{N} \downarrow N$ ligation) two strategies have been implicated to increase the selection pressure in favor of 3'-5' bond formation. In one strategy, the enzyme region was designed offset from the ligation junction, such that the new RNA linkage is formed within a duplex region. The strategy was successful and a set of enzymes were selected using this strategy which all form exclusively native 3'-5' bonds but their reaction rates and generality were suboptimal. 8AY13 was the fastest enzyme with the $k_{\text {obs }} 0.008 \mathrm{~min}^{-1}$ at $37{ }^{\circ} \mathrm{C}$ and $40 \mathrm{mM} \mathrm{Mg}{ }^{2+}$, $\mathrm{pH} 9.0(41 \%$ yield at $3 \mathrm{~h}$ incubation $)^{30}$.

In a second strategy, which is also explained in the sub chapter 1.1, an additional stringent positive selection was introduced to the selection rounds. DNA species which were capable of ligation and were up-shifted in PAGE separation were cleaved with 8-17 which was a previously selected RNA cleaving deoxyribozyme. 8-17 is only capable of cleaving native 3'-5' linear bonds. Therefore, the DNA species which formed the native 3'-5' linear bond were cleaved and thereby their electrophoretic mobility was increased in the next round of PAGE separation. Only the RNA-DNA conjugates which passed both positive selections were enriched by PCR, afterwards ${ }^{13}$. Two deoxyribozymes have been selected by this strategy; 9DB1 and 7DE5 which are capable of ligation for D $\downarrow R A$ and $A \downarrow R$ junctions where $D$ denotes for $A, G$ or $U$ and $R$ stands for purines $A$ or $G^{31}$. The 9DB1 reaction rate in presence of $40 \mathrm{mM} \mathrm{Mg}^{2+}$, $\mathrm{pH} 9.0$ at $37^{\circ} \mathrm{C}$ was $0.036 \mathrm{~min}^{-1}$. The reaction rate for 7DE5 in presence of $1 \mathrm{mM} \mathrm{Zn}{ }^{2+}, \mathrm{pH} 7.5$ at $23^{\circ} \mathrm{C}$ was $0.019 \mathrm{~min}^{-1}$. The two deoxyribozymes were shown to effectively ligate naturally relevant RNA sequences to form Tetrahymena group I intron P4-P6 domain (160 nt) and G-riboswitch (72 nt).

\subsection{DNA-cleaving deoxyribozymes}

The first DNA cleaving deoxyribozyme was selected during an in vitro selection experiment which aimed to discover a deoxyribozyme for amide bond cleavage (10MD5 family). Out of all 44 active DNA species no one was able to cleave any amide bond but rather their cleavage sites were inside the double helical DNA binding arms ${ }^{32}$. 10MD5 was characterized further for its substrate requirements and dependence on metal ions and pH. 10MD5 is active in presence of $\mathrm{Mn}^{2+}$ and narrow range of $\mathrm{Zn}^{2+}$ concentration and $\mathrm{pH}^{32}$. Later on deoxyribozymes were reported with better tolerance regarding $\mathrm{pH}^{33}$ and substrate sequence generality ${ }^{34}$.

More recently two additional classes of $\mathrm{Zn}^{2+}$ dependent DNA hydrolyzing deoxyribozymes were selected by Breaker and coworkers ${ }^{35}$. I-R3 was chosen as the representative of their selection which was shown to be solely active with $\mathrm{Zn}^{2+}$. Other bivalent metal ions such as $\mathrm{Mg}^{2+}, \mathrm{Mn}^{2+}, \mathrm{Cu}^{2+}, \mathrm{Co}^{2+}, \mathrm{Cd}^{2+}$ and $\mathrm{Ni}^{2+}$ did not activate the I-R3 catalysts.

Silverman and coworker attempted in vitro selection for lanthanide assisted DNA hydrolysis in presence of $\mathrm{Zn}^{2+}$. The in vitro selection resulted in numerous deoxyribozymes which were active in the presence 
of $\mathrm{Zn}^{2+}$ and micromolar concentrations of lanthanides e.g. $\mathrm{Ce}^{3+}, \mathrm{Eu}^{3+}$, and $\mathrm{Yb}^{3+}$. Examples include 7YK and $6 \mathrm{YJ}$ families of deoxyribozymes. Individual members of these families were active in the presence of combinations of $\mathrm{Zn}^{2+}$ and various lanthanide ions. Neither of the enzymes were active in the absence of lanthanide ions but $\mathrm{Zn}^{2+}$ could be omitted from the reactions of members of $\mathrm{Yb}^{3+}$ dependent enzymes i.e. 6YL4 and 6YL24 ${ }^{36}$. The two enzymes 6YL4 and 6YL24 are the first examples of DNA hydrolyzing deoxyribozymes which are independent from $\mathrm{Zn}^{2+}$.

\subsection{DNA-ligating deoxyribozymes}

For DNA catalyzed DNA ligation, one of the DNA strands needs to be activated in advance. In 1995, a deoxyribozyme was selected which was able to form the natural 3'-5' phosphodiester bond using an activated 3'-phosphorimidazolide substrate ${ }^{37}$. To ligate two DNAs with 3'- and 5'- hydroxyl groups, a three step strategy is proposed using deoxyribozymes for each step. There has been a report on catalytic DNAs being capable of self-phosphorylation. The source of activated phosphate for these enzymes was any of the standard triphosphorylated ribonucleotides or deoxyribonucleotides ${ }^{38}$. Additionally, several self-capping DNAs were selected and reported. Capping deoxyribozymes catalyze the transfer of the AMP moiety of ATP to their $5^{\prime}$-terminal phosphate group, thereby forming a 5',5'-pyrophosphate linkage $^{39}$. Furthermore, self-ligating deoxyribozymes, which facilitate formation of 3',5' phosphodiester bond by catalyzing nucleophilic attack of their 3'-OH to their 5'-adenylated end and release of AMP was reported in $2004^{40}$. The combination of these three catalytic DNAs would allow ligation of two DNA strands in vitro (Fig 1.5-1)

Formation of a branched DNA by a deoxyribozyme is also reported. As an example, 8LV deoxyribozyme family ${ }^{42}$ use a DNA containing a single ribonucleotide as the acceptor strand and an adenylated DNA as the donor strand to form a 2',5'- branched DNA in presence of $\mathrm{Mn}^{2+}$.

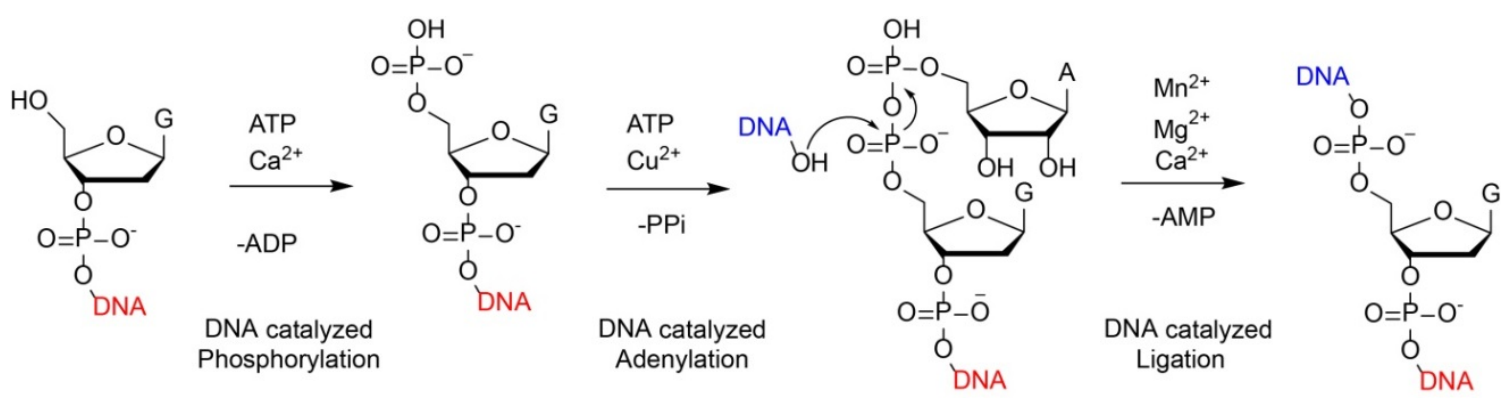

Fig 1.5-1 Three step DNA ligation, using 5'- and 3'-OH bearing DNA strands catalyzed by three different deoxyribozymes. In the first step the 5 ' end of the DNA substrate is phosphorylated in presence of $\mathrm{Ca}^{2+}$ and using ATP. In the second step the monophosphorylated substrate is adenylated and thereby activated using ATP and in presence of $\mathrm{Cu}^{2+}$. The last step is the ligation of two DNA strands upon release of the AMP. Figure adapted from ${ }^{41}$ 


\subsection{Peptide-modifying deoxyribozymes}

Amino acid side chains of peptides are another set of deoxyribozyme substrates. In 2008, several deoxyribozymes have been reported which were able to catalyze formation of a nucleopeptide linkage by activating the $\mathrm{OH}$ group present in amino acid side chains (i.e. in Tyr, Ser) for nucleophilic attack to the $\alpha$-phosphate of a triphosphorylated RNA. The amino acid with the 2'-OH nucleophile was flanked by DNA strands which were designed to hybridize to the DNA binding arms and participate in formation of a three helix junction (3HJ) structure to facilitate catalysis. Tyr1 was selected as the representative of these enzymes ${ }^{43}$. In 2011, a new selection was performed to discover DNA enzymes which are able to perform the same type of reaction but for a nucleophile $-\mathrm{OH}$ of a Tyr which was embedded between amino acids instead of nucleotides. 15MZ36 is a representative of this set of selection (Fig 1.6-1 A) ${ }^{17}$.

Furthermore, in 2012, two enzymes were reported with the ability to distinguish phosphorylated and non-phosphorylated Tyr (and/or Ser) within a peptide chain; 8VM1 and 8VP1. These enzymes catalyze formation of a linkage between phosphorylated Tyr (or Ser) with $\alpha$-phosphate of a triphosphorylated RNA (Fig 1.6-1 B) ${ }^{44}$. More recent, a phosphatase deoxyribozyme has been reported; 14WM9 ${ }^{16}$. 14WM9 catalyzes $\mathrm{Zn}^{2+}$ dependent phosphomonoester hydrolysis of tyrosine and serine side chains (Fig 1.6-1 C). For 14WM9 in vitro selection, the deoxyribozyme $15 \mathrm{MZ36}^{17}$, was applied to ligate a DNA strand to the dephosphorylated products and therefore tag the active molecules by an increase in their molecular weight.

\subsection{Deoxyribozymes with small molecules as substrates}

In vitro selections for deoxyribozymes which catalyze modifications of small molecules are challenging due to difficulties of the capture steps. In the other hand catalysis of organic chemical reactions is of interest for chemists. Strategies have been developed for in vitro selection of known reactions in organic chemistry such as Diels-Alder or Friedel-Crafts.

Diels-Alder reaction forms a carbon- carbon bond between a conjugated diene and a substituted alkene ${ }^{45}$. The deoxyribozyme, DAB2 $2^{46}$ has been selected by Silverman and coworkers to catalyze the reaction in presence of $\mathrm{Ca}^{2+}, \mathrm{Mn}^{2+}$ or $\mathrm{Mg}^{2+}$ at $\mathrm{pH}$ 7.5. Friedel-Crafts reaction attaches substituents to an aromatic ring. There are two main types of Friedel-Crafts reactions: alkylation reactions and acylation reactions. Friedel-Craft alkylation reaction using an indole and acyl imidazole was subjected for an in vitro selection ${ }^{47}$. The selected enzymes were dependent on micromolar concentration of $\mathrm{Cu}^{2+}$ at $\mathrm{pH} 6.5$. 


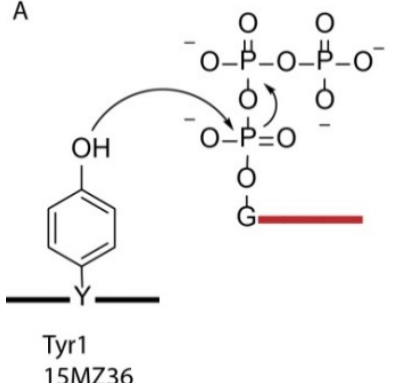

B

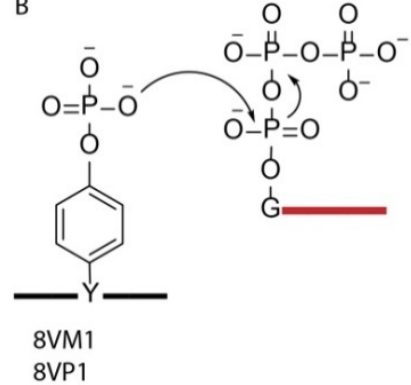

C

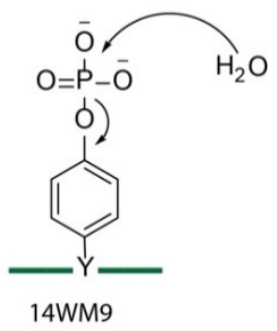

Fig 1.7-1 Schematic depiction of three types of reaction catalyzed by DNA on peptide substrates. A) Nucleopeptide bond formation catalyzed by Tyr1 and 15MZ36. The amino acid Tyr is embedded in DNA strand (Tyr1) or peptide chain (15MZ36). B) phosphor-anhydride bond formation catalyzed by 8VM1 and 8VP1. For 8VP1 a phosphorylated serine is accepted as well. C) Phosphatase reaction catalyzed by 14WM9. The red lines indicate triphosphorylated RNAs. The black lines indicate either DNA or peptide chains. The green lines represent peptide chains.

In addition to Diels-Alder and Friedel-Craft deoxyribozymes, in vitro selection revealed DNA aptamers which bind porphyrins such as $N$-methylmesoporphyrin IX (NMM) and catalyze their metalation with $\mathrm{Cu}^{2+}$ or $\mathrm{Zn}^{2+48}$. It has been discovered that Fe (III)-porphyrin IX (hemin) competitively binds to the same DNA aptamers. Hemin, is a cofactor of variety of enzymes e.g. peroxidases and monooxygenases and has intrinsic oxidation activity ${ }^{49}$. The oxidative activity of hemin was enhanced upon binding to these DNA aptamers which encouraged scientists to apply the deoxyribozyme peroxidase ${ }^{50}$ in biosensors.

\subsection{Generality of deoxyribozymes}

Most of deoxyribozymes are involved in formation or breaking of a P-O bond. Examples include all RNA ligating or cleaving deoxyribozymes, phosphorylating ${ }^{38}$, adenylating ${ }^{39}$ and DNA ligating ${ }^{40}$ deoxyribozymes and also all the deoxyribozymes reported for peptide modification. DNA enzymes are not limited to catalysis of formation and hydrolysis of P-O bonds. For example Diels-Alder deoxyribozyme (DAB22) catalyzes formation of a C-C bond ${ }^{46}$. UV1A and UV1C deoxyribozymes which catalyze thymine dimer photo reversion, break a C-C bond ${ }^{51,52}$. Although in vitro selection is reported to be challenging when an $\mathrm{N}$ atom is the nucleophile or the leaving group ${ }^{53}$ there are reports for successful reductive amination (formation of C-N bond) ${ }^{54}$ or breaking an N-glycosidic bond of a 5' terminal guanosine of a DNA substrate ( C-N bond, $10-28{ }^{55}$ and $10 \mathrm{FN} 10^{56}$, catalyzing deglycosylation).

One common question about catalytic nucleic acids is when a specific sequence of RNA (a ribozyme) can catalyze one type of reaction, is the same sequence of DNA (possibly with a couple of mutations) capable of catalyzing the same reaction. Another common question is what are the rate differences between a deoxyribozyme and a ribozyme that both catalyze the same reaction type. The reports on the nucleic acid enzymes performing Diels-Alder reaction addressed such questions. The first report for Diels-Alder reaction by nucleic acid catalysis was on a ribozyme (39M49) ${ }^{57,58}$. Based on the 39M49 sequence Chandra and Silverman started new in vitro selections with a library of random sequence which was biased to 39M49 sequence. Additionally they performed an un-biased selection for the Diels- 
Alder reaction. The best outcome of the selection, DAB22 (introduced in section 1.7), was from the biased pool and showed similar activity to the ribozyme 39M49 but it had 13 mutations relative to it. The mfold-predicted secondary structures of the two enzymes were quite dissimilar. Therefore, it was concluded that although DAB22 has been selected from the biased pool, it is a new catalytic sequence ${ }^{46}$.

\subsection{Applications of deoxyribozymes}

Currently, most of the applications of deoxyribozymes e.g. DNA computing and molecular logic gate designs $^{18}$ or in vivo applications ${ }^{59}$ in the direction of therapeutics ${ }^{60,61}$ are based on RNA cleaving deoxyribozymes. For applications of deoxyribozymes in biological context, the main challenge is delivery and stability of the deoxyribozymes. In situ production of single stranded DNAs has been reported using an expression vector containing Moloney mouse leukemia virus reverse transcriptase (MMLV-RT) and the deoxyribozyme sequence under a T7 promoter. The deoxyribozyme sequence was transcribed to RNA and subsequently reverted to the single stranded stage by MMLV-RT ${ }^{62}$. Alternatively, in situ synthesis of deoxyribozymes was achieved by insertion of the deoxyribozyme sequence inside M13 bacteriophage DNA which provided amplification of the deoxyribozyme sequence using rolling circle amplification (RCA) ${ }^{63}$.

Aptamers are single stranded DNA or RNA molecules which form a unique conformation with specific recognition abilities to target molecules. Aptamers are in analogy to protein antibodies and are applied in research and therapeutics ${ }^{64}$ with the same logic but have advantageous aspects to protein antibodies. Aptamer and deoxyribozymes are easily synthesized compared to protein enzymes. They can adapt the active fold even after denaturation by heat or absence of the required metal ions, when the amenable temperature and metal ions are present again. Additionally, aptamers can respond to presence of small targets e.g. non-chelated metal ions such as $\mathrm{Pb}^{2+}$ or $\mathrm{Mg}^{2+}$, while in the other hand protein antibodies are limited when the target molecule is not immunogenic. In vitro synthesis is another advantage of nucleic acid aptamers and deoxyribozymes since production of protein antibodies gets limited when the target molecules are toxic.

Aptamers and deoxyribozymes are employed as part of biosensors. Biosensors consist of recognition and signal transduction modules which are connected to a proper readout system. Aptamers only serve as the recognition motif while deoxyribozymes can perform both tasks (recognition and signal transduction). When a deoxyribozymes is used, the target molecules must either accelerate or inhibit the enzyme $^{65}$. Until now a vast number of aptamers, ribozymes and deoxyribozymes have been selected in vitro for a wide range of target molecules with different target specificity and sensitivity. Ellington and co-workers have created a searchable online aptamer database. The database was started with 239 published aptamers and unnatural ribozymes discovered until $2004^{66}$. More recently a new database for DNA and RNA aptamers has been created with currently (July 2013) 1800 entries ${ }^{67}$. 
There are three types of biosensors based on their readout systems i.e. fluorescent, colorimetric or electrochemical readout systems. Peroxidase deoxyribozyme ${ }^{68}$ is an example which is combined with a fluorescent detector. Several reports are available which use peroxidase deoxyribozyme e.g. for detection of $\mathrm{Pb}^{2+}$ in blood samples ${ }^{69}$.

The peroxidase deoxyribozyme catalyzes oxidation of non fluorescent substrate to highly fluorescent substrates by $\mathrm{H}_{2} \mathrm{O}_{2}{ }^{70,71}$. It is employed in the same way that protein peroxidase HRP (horseradish peroxidase) is applied in ELISA ${ }^{68}$. 2,2'-azino-bis(3-ethylbenzothiazoline-6-sulfonic acid) (ABTS) ${ }^{72,73}$, 3,3',5,5'-tetramethylbenzidine sulfate $(\mathrm{TMB})^{74}$ or Amplex UltraRed (AUR) ${ }^{69}$ are examples of the substrates used for peroxidase activity in biosensors. The enzyme performs multi-turnover reaction which allows signal amplification.

First generations of colorimetric biosensors were based on $\mathrm{Pb}^{2+}$ dependent $\mathrm{RNA}$ cleaving deoxyribozymes and metallic nanoparticles. Metallic nanoparticles have high extinction coefficient and distance-dependent optical properties. Therefore their aggregation level can be monitored by UV-Vis spectroscopic methods. In an 8-17 based biosensor reported by Liu et al. in 2003, single stranded DNAs were immobilized on gold nanoparticles (AuNPT). RNA substrates of 8-17 deoxyribozymes had an overhang complementary to immobilized DNAs (Fig 1.9-1). Before RNA cleavage formation of aggregates of AuNPs was possible because the RNA-deoxyribozyme complexes bridged the AuNPs. Nanomolar concentrations of $\mathrm{Pb}^{2+}$ resulted in cleavage of the RNA substrates and dispersion of the AuNPT $^{75}$.

Using 8-17 for detection of $\mathrm{Pb}^{2+}$ requires that the enzymes is exclusively active in the presence of $\mathrm{Pb}^{2+}$ but no other metal ions. Although, it has been shown that the enzyme 8-17 is active in the presence of absence $4 \mathrm{M} \mathrm{Li}^{+}$when no $\mathrm{Pb}^{2+}$ is present, the reaction rate was $\sim 200$ '000 fold slower for $4 \mathrm{M} \mathrm{Li}^{+}$. This is considered as an excellent selectivity of $\mathrm{Pb}^{2+}$ over the large excess of monovalent metal ions ${ }^{76}$.

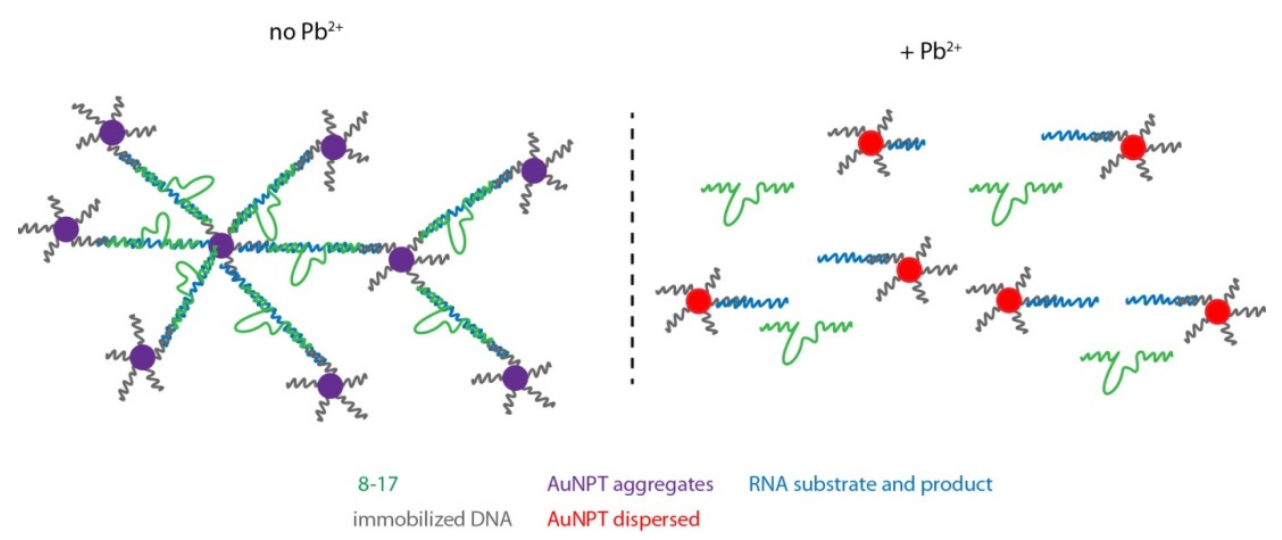

Fig 1.9-1 Colorimetric biosensor for $\mathrm{Pb}^{2+}$ detection. Single stranded DNAs (gray) were immobilized on the surface of gold nanoparticles (AuNPTs). In the absence of $\mathrm{Pb}^{2+}$ the 8-17 deoxyribozymes (green) forms bridges between AuNPTs and causes aggregation of AuNPTs. The DNA-RNA complex bridges are destabilized upon presence of $\mathrm{Pb}^{2+}$ and cleavage of the RNA substrates. Dispersion of the AuNPTs is detectable by colorimetric methods. Figure is adapter from ${ }^{75}$. 
Electrochemical sensing has advantages over fluorescent or colorimetric methods. The instrumental setup for electrochemical sensors cost less and easy signal amplification results in higher sensitivity. In a case report, $\mathrm{Pb}^{2+}$ dependent 8-17 deoxyribozyme was immobilized onto a gold electrode via its 5' end. A methylene blue (MB) group was attached to the 3' end of the enzyme. While the RNA substrate was hybridized to the enzyme, the stiff RNA-DNA duplex of the substrate-enzyme binding arms kept the MB group away from the surface and therefore electron transfer between the surface and MB was reduced. Upon presence of nanomolar concentration of $\mathrm{Pb}^{2+}$ the $\mathrm{RNA}$ substrate was cleaved and released and the electron transfer rate between the MB and the surface was increased ${ }^{77}$.

Most of the applications described above were based on RNA-cleaving deoxyribozymes or the peroxidase deoxyribozyme. However, RNA-ligating deoxyribozymes have the potential for future applications. Deoxyribozymes application can be expanded for RNA labeling and RNA functionalization based on RNA-ligating deoxyribozymes ${ }^{78}$. Labeled RNAs are used to study functional and regulatory RNAs. Current RNA labeling methods directly incorporate modifications via solid-phase synthesis and therefore are limited in the length of the synthesized labeled RNA fragment. Usually, for RNA molecules larger than 50 nucleotides, several smaller labeled RNA fragments are prepared and ligated via T4 RNA or T4 DNA ligases. In a new approach by our group the 3'-5' ligating deoxyribozyme 9DB1 was applied to ligate labeled fragments of SAM III riboswitch ${ }^{79}$.

During the last decade, lots of regulatory RNAs have been discovered and extensively studied. siRNAs and miRNAs in eukaryotic cells function in transcriptional or post-transcriptional level, based on complete or partial complementarity to the target mRNA ${ }^{80,81}$. Studies have also shown small RNA dependent regulation in prokaryotes and archaea ${ }^{82}$. Small RNAs (sRNAs) interfere with transcription or translation of mRNAs. sRNAs are responsible for several aspects of bacterial life e.g. environmental adaptation, virulence and sensation of metabolites ${ }^{83}$. Another class of RNA regulators that are involved in sensation of metabolites is riboswitches. Binding of the riboswitches to their ligands stabilizes conformations which trigger transcriptional or translational initiation or termination, causing on or off switches for the downstream genes ${ }^{84}$. Riboswitches are also involved in RNA stabilization e.g. glucosamine-6-phosphate activated riboswitch (glmS) $)^{85}$ and eukaryotic cellular processes such as alternative splicing e.g. thiamin pyrophosphate (TPP) riboswitch ${ }^{86,87}$.

An additional potential applications for deoxyribozymes which are able to form 2',5' branched RNA is synthesis of lariat RNAs. Lariat RNAs are intermediate products of splicing reactions by spliceosome ${ }^{88}$ or Group II introns ${ }^{89}$. Structurally, a lariat RNA is a 2',5' branched RNA in which the strand extended from 2' of the branch site loops around to be the same RNA strand which provides the branch site (Fig 1.9-2). For in vitro synthesis of lariat RNAs two strategies are available: 1) two-step strategy which consists of formation of an inter-molecular 2',5' branched RNA and subsequent closure of the loop by T4 RNA ligase. $^{90}$ 2) one-step strategy, using a deoxyribozyme which tolerates the loop closure. For the 
latter, to form a lariat RNA, the provided RNA substrate need to serve as both donor and acceptor and binds to both binding arms of the deoxyribozyme.
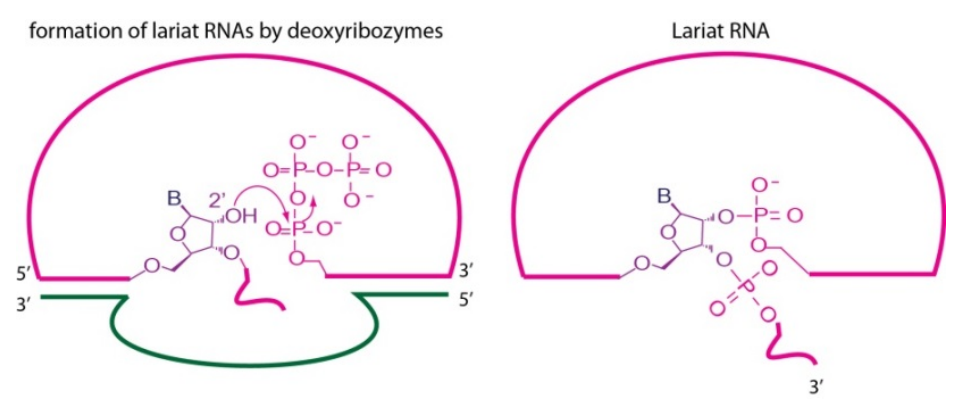

Fig 1.9-2 Deoxyribozymes mediated lariat formation. For lariat formation, the donor RNA which is normally hybridized to the right binding arm of the deoxyribozyme, loops around and hybridizes to the left binding arm of the deoxyribozyme to provide the nucleophile and fulfill the task of the acceptor RNA.

Lariat RNA formation is intrinsically more difficult compared to simple 2',5' branched RNA. The loop closure may constrain the appropriate hybridization of regions of the RNA which must bind to deoxyribozyme. Secondly, the loop present in the lariat RNA may evolve secondary and tertiary structures interfering with the deoxyribozyme activity. Thirdly, partial complementarity between the loop sequence and the catalytic core could interrupt formation of the active fold. A large number of 2', 5' branch forming deoxyribozymes are discovered, with the most effective one being 7S11, raising the hope for in vitro formation of lariat RNAs ${ }^{24}$ but only one enzyme, 6BX22, is reported being capable of formation of lariat RNAs using biologically related RNA substrates ${ }^{26}$.

In summary, the considerably emerging field of nanobiotechnology can benefit from advances in the field of DNA-catalysts. Understanding the basics of a system (here DNA catalysis) promotes further development of the application of that system. Functional characterization of deoxyribozymes would open new routes to improve deoxyribozyme applications.

\subsection{Functional characterization of (deoxy) ribozymes}

Functional studies and understanding the mechanism and sequence requirements of deoxyribozymes promotes engineering and expanded application of deoxyribozymes in molecular biology and nanotechnology. Little is known about the mechanism of deoxyribozymes from chemical point of view and our understanding about three dimensional folding of deoxyribozymes are limited. There are programs e.g. "mfold" to predict possible secondary structures. "mfold" is based on Michael Zuker algorithm which was proposed in $1981^{91}$ to calculate minimum free energy (MFE) structure of a given DNA or RNA sequence. MFE algorithm is used by several other secondary structure prediction programs such as "RNAfold"92. Mathematical MFE algorithm in combination to constraints from biochemical data such as DNase /RNase cleavage patterns or selective 2'-hydroxyl acylation analyzed by primer extension (SHAPE) lead to more accurate prediction of secondary structures, implicated in "RNAstructure" program and web server ${ }^{93}$. Most of the available methods are only useful for RNA and 
are not directly applicable for functional DNAs. Software, methods and strategies should be developed for investigation of the structure and function of catalytic DNAs.

Crystal structure of the RNA cleaving enzyme 10-23 in complex with its inactivated substrate (2'-OMe modification at the cleavage site) was reported in 1999 by Joyce and coworkers ${ }^{94}$. The reported structure consisted of two 10-23 DNA and two RNA substrates forming four stems of DNA binding arms and RNA substrates. The catalytic cores of the two 10-23 DNAs were hybridized together with two four way junction on both sides of the hybridized region forming the fifth stem (Fig 1.10-1). 10-23 deoxyribozyme contains the sequence GCTAGCT in its catalytic core. This sequence is partly palindromic. In the reported structure the two four-way junctions provided an additional extra $\mathrm{A}$ in the 5 ' of the sequence and resulted in formation of an 8-nucleotides long stem in the catalytic core of the 2:2 DNA-RNA complex. Such structure was not in agreement with the biochemical data. The rate constant for the enzyme 10-23 was reported to be independent from the enzyme concentration. Therefore, the crystalized specimen which required two DNA could not resemble the active structure.
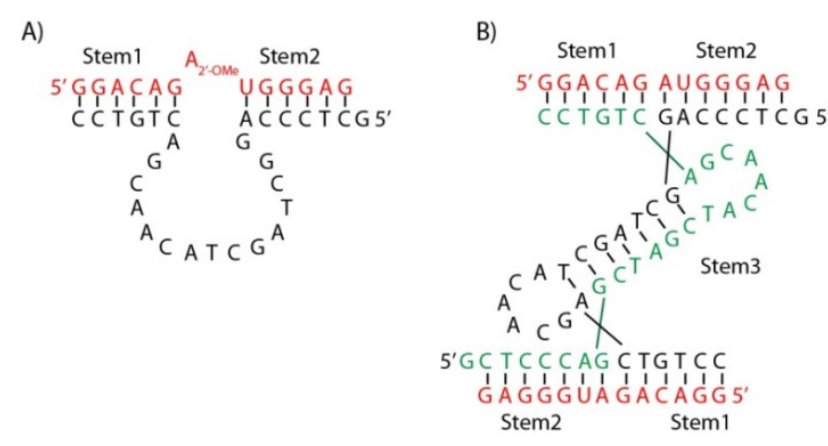

Fig 1.10-1 Crystal structure of 10-23 deoxyribozyme A) The RNA cleaving deoxyribozyme 10-23 and the inactive substrate used for crystallization. The adenosine at the cleavage site was inactivated with replacement of its 2'$\mathrm{OH}$ by 2'-OMe. B) The schematic depiction of crystal structure of 10-23. Two 10-23 DNA and two RNA substrates participated in formation of extended stems. Each substrate was hybridized to both DNA binding arms upstream and downstream of the cleavage site. In this scheme, substrates are shown in red. DNAs are shown in green or black.

\subsubsection{Role of metal ions}

Ribozymes and deoxyribozymes are highly dependent on metal ions for folding ${ }^{95,96}$ and catalysis ${ }^{97,98}$. Most of the natural ribozymes are dependent on both $\mathrm{K}^{+}$and $\mathrm{Mg}^{2+}$ as the two abundant metal ions in contact to nucleic acids in cellular environment. Cytosolic concentrations of free $\mathrm{Mg}^{2+}$ and $\mathrm{K}^{+}$are $0.2-$ $1 \mathrm{mM}$ and $140 \mathrm{mM}$ respectively ${ }^{99,100}$. In vitro selected (deoxy) ribozymes in the other hand are normally selected in presence of sodium chloride and a combination of bivalent or trivalent metal ions and are mostly dependent on bivalent metal ions such as $\mathrm{Mg}^{2+}, \mathrm{Mn}^{2+}$ and $\mathrm{Zn}^{2+}$. Specific types of deoxyribozymes are also reported to strictly being active in presence of trivalent lanthanide ions ${ }^{36}$.

To neutralize the negatively charged backbone of nucleic acids a "metal ion atmosphere" must surround (deoxy) ribozymes. Individual metal ions have different commitment. Some metal ions are only transiently present and are exchanged with the metal ions in the bulk solution. Diffusely bound ${ }^{101}$ metal ions are normally not detectable in X-ray crystallographic data. The charge-screening metal ions decrease the electrostatic repulsion between the phosphate groups and allow compact folding of the nucleic acid to its active structure. 
In the first hydration shell of charged molecules (metal ions or phosphate groups of DNA), the water molecules are strongly arranged with pronounced orientations towards or away from the ion ${ }^{102}$. For example $\mathrm{Mg}^{2+}$ predominately binds to six water molecules in an octahedral geometry. For diffusely bound metal ions water shell maintains intact and the anionic and cationic charges interact via Columbic interactions through solvent ${ }^{103}$. There are two types of site bound metal ions. Outer sphere coordination is defined when the water shells of a metal ion and a phosphate group or any other electronegative functional group of nucleic acids share one or more water molecule. Inner sphere binding is defined when the two charges are in direct contact without an intervening water molecule (Fig 1.10-2). Dehydration of the two charges causes an enthalpic burden on the system which is compensated by consequent direct interaction of the two charges ${ }^{104}$.

A) Diffuse binding

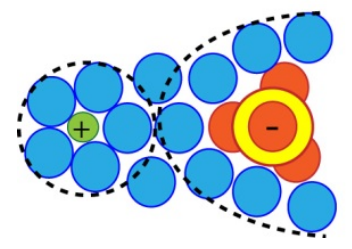

B) Outer sphere binding

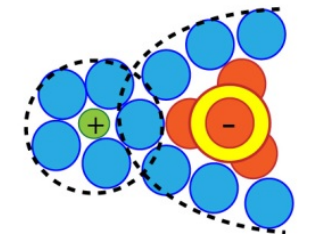

C) Inner sphere binding

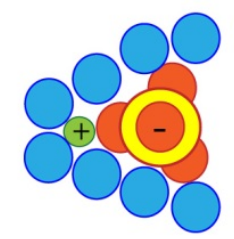

Fig 1.10-2 Modes of metal ion binding to nucleic acids A) diffuse binding in which the solvation shell of the ion and its phosphate ligand remain intact $B$ ) outer sphere complex in which the ion and its ligand share their solvation shells $\mathrm{C}$ ) inner sphere complex in which the ion and its ligand make direct contact without any water bridge. $\mathrm{Mg}^{2+}$ ions are green; water molecules are blue, phosphorous atoms are yellow and oxygen atoms are red. Water shells are defined by dotted lines. The figure is adapter from ${ }^{104}$.

Strong dependence of (deoxy) ribozymes to metal ions raised ideas that suggest (deoxy) ribozymes are metaloenzymes. However, it is possible that the metal ions are only important for the formation of the active fold and are not present in the catalytic center and the reaction is facilitated by general acid base catalysis, destabilization of the substrates or stabilization of the charge or shape of the transition state ${ }^{105}$. Several investigations on the dependence of (deoxy) ribozyme on metal ions proved that catalytic DNAs or RNAs are not necessarily metaloenzymes.

Despite general dependence of (deoxy) ribozymes on bivalent metal ions, there are examples of (deoxy) ribozymes which their activity is solely dependent on monovalent metal ions. Deoxyribozymes which have a G-quadruplex in their active structure are only dependent on monovalent metal ions. Thymine dimer repair deoxyribozymes UV1A and UV1C are two examples which their exclusive dependence on $\mathrm{K}^{+}$and $\mathrm{Na}^{+}$but not on $\mathrm{Li}^{+}$and bivalent metal ions allowed prediction of presence of a G-quadruplex in their structure ${ }^{106}$. Additional supporting data such as DMS probing of the folded UV1 $\mathrm{A}^{51}$ proved this prediction. Involvement of a G-quadruplex in UV1C was further confirmed by 6-methylisoxanthopterin $(6 \mathrm{MI})^{107}$ substitution for single guanine residues within UV1C. Additionally, 6MI substitution improved the UV light sensitivity of the modified UV1C to violet region $(345 \mathrm{~nm})^{52}$.

Additionally, Geyer and Sen performed in vitro selection of RNA cleaving ribozymes which were active only in the presence of monovalent metal ions. The separation steps for in vitro selection were 
performed in the presence of $1 \mathrm{M} \mathrm{Na}^{+}$at $\mathrm{pH} 7.0$ but the best activity was observed with $0.5 \mathrm{M} \mathrm{Na}^{+}$. They have shown addition of $\mathrm{Zn}^{2+}, \mathrm{Pb}^{2+}, \mathrm{Mg}^{2+}$ and $\mathrm{Ca}^{2+}$ does not significantly improve the reaction ${ }^{105}$.

Hammerhead and hairpin ribozymes are shown to be active in presence of high concentrations of monovalent metal ions $\left(4 \mathrm{M} \mathrm{Li}^{+}\right)$or even non-metallic cations $\left(4 \mathrm{M} \mathrm{NH}_{4}^{+} \text {or }\left[\mathrm{Co}\left(\mathrm{NH}_{3}\right)_{6}\right]^{+3}\right)^{108,109}$. Hairpin ribozyme was 10 -fold slower in presence of $4 \mathrm{M} \mathrm{Li}^{+}$when compared to its activity in presence of $10 \mathrm{mM} \mathrm{Mg}^{2+}$. Grasby and coworkers ${ }^{109}$ have proved enzymatic activity of hairpin ribozyme in presence of Cobalt (III) hexamine and proposed that metal ions play a passive role in the hairpin ribozyme catalysis and are probably required only for structural purposes.

The in vitro selected RNA cleaving deoxyribozyme 8-17 was also shown to be active in presence of 4 $\mathrm{M} \mathrm{Li}^{+}$or $\mathrm{NH}_{4}^{+}$but not with any other monovalent metal ion or cobalt hexamine ${ }^{76}$. However its activity was 1000 -fold slower in presence of $4 \mathrm{M} \mathrm{Li}^{+}$compared to its activity in presence of $10 \mathrm{mM} \mathrm{Mg}{ }^{2+}$. The 8-17 enzymatic activity in presence of $4 \mathrm{M} \mathrm{Li}^{+}$was $\sim 200$ ’000-fold slower than its activity in presence of $100 \mu \mathrm{M} \mathrm{Pb}^{2+}$, making the 8-17 deoxyribozyme a great choice as a lead sensor.

In the absence of three dimensional structures, spectroscopic techniques are helpful to investigate metal ion binding sites and their role in catalysis. Many of the metal ions involved in deoxyribozyme folding and catalysis such as $\mathrm{Na}^{+}, \mathrm{K}^{+}, \mathrm{Zn}^{2+}, \mathrm{Pb}^{2+}$ and $\mathrm{Mg}^{2+}$ do not have rich spectroscopic properties. Although $\mathrm{Mn}^{2+}$ is paramagnetic and can be applied as a probe in EPR studies ${ }^{110}$, it is required in mM ranges for deoxyribozyme activities, and high levels of free $\mathrm{Mn}^{2+}$ in solution can bury the signal of bound $\mathrm{Mn}^{2+}$. Lanthanides are spectroscopically rich elements and can be used for such studies.

\subsubsection{Lanthanides and nucleic acids}

The fifteen lanthanide elements, [Xe] $4 \mathrm{f}^{\mathrm{n}} 5 \mathrm{~s}^{2} 5 \mathrm{p}^{6}$, are known as rare-earth elements. Lanthanides are commonly denoted as Ln and possess atomic numbers from 57 to 71 . Lanthanides consist of Lanthanum (La), Cerium (Ce), Praseodymium (Pr), Neodymium (Nd), Promethium (Pm), Samarium(Sm), Europium (Eu), Gadolinium (Gd), Terbium (Tb), Dysprosium (Dy), Holmium (Ho), Erbium (Er), Thulium (Tm), Ytterbium (Yb), Lutetium (Lu).

The filling of the $4 \mathrm{f}$ orbitals from La to $\mathrm{Lu}$ is accompanied by a smooth decrease in ionic radii and $\mathrm{pKa}$ of the bound water to the metal ions of the series ${ }^{111}$. The decrease in lanthanides' ionic radii is known as "lanthanide contraction". Considering the effective ionic radii reported by Shannon and Prewitt in $1976^{112}$, in the beginning of the lanthanide series eight-coordinate ionic radii of $\mathrm{La}^{3+}$ and $\mathrm{Ce}^{3+}$ are 1.160 and $1.143 \AA$. The ionic radii of the last two members of the lanthanide series, $\mathrm{Yb}^{3+}$ and $\mathrm{Lu}^{3+}$, are 0.985 and $0.977 \AA$ respectively. The Shannon ionic radius of biologically abundant $\mathrm{Mg}^{2+}$ ion with coordination number of eight is $0.89 \AA$. Because of similarities of the size of lanthanides and magnesium ions, lanthanides are considered as useful probes of metal binding sites for biomolecules with $\mathrm{Mg}^{2+}$ binding sites ${ }^{113}$. 
For lanthanides, the geometry of the first coordination shell is not determined by the type of the lanthanide ions but mainly by the electrostatic attraction of charged ions of solvent molecules or by electrostatic and steric repulsion between the solvent molecules ${ }^{36}$. The coordination number of the aquatic trivalent lanthanides changes from nine for the lighter ions $\left(\mathrm{La}^{3+}\right.$ to $\left.\mathrm{Nd}^{3+}\right)$ to eight for the heavier ions $\left(\mathrm{Gd}^{3+} \text { to } \mathrm{Lu}^{3+}\right)^{114}$.

As a consequence of $4 \mathrm{f}$ orbitals shielding by filled $5 \mathrm{~s}$ and $6 \mathrm{p}$ orbitals, direct photoexcitation of lanthanides give rise to extremely weak luminescence upon electric dipole transitions between states with configuration of $4 \mathrm{f}^{\mathrm{n}}$. In aqueous solution, the weak signal is further suppressed upon non-radiative relaxation through $\mathrm{OH}$ oscillators of water ${ }^{115}$. Therefore, when free in water, due to the low molar absorptions of lanthanides they can only be effectively excited by lasers. Lanthanide luminescence can be significantly enhanced by chelating ligands in a process called "sensitization".

In sensitized luminescence of lanthanides a ligand acts as antenna or sensitizer. The ligand is excited and passes its energy efficiently and non-radiatively to the lanthanide ion. The hard Lewis acidity of the lanthanide ions results a specific preference for ligands with atoms that can act as hard Lewis bases. Therefore, combinations of amines and carboxylic groups ${ }^{116}$ are commonly used in lanthanide ligands ${ }^{117}$ such as $1,4,7,10$ - tetrakiscarboxymethyl-1,4,7,10-tetraazacyclododecane (DOTA) ${ }^{118}$.

Sensitized lanthanide luminescence was employed in wide range of applications. As an example, a short hairpin RNA was studied using sensitized luminescence of $\mathrm{Tb}^{3+}$ in complex with DOTA ${ }^{119}$. Ligand mediated $\mathrm{Tb}^{3+}$ luminescence was also used for double stranded DNA detection ${ }^{120}$. Terbium-complex with difloxacin (DFX) was shown to specifically enhance luminescence intensities with double stranded DNA while single stranded DNA increased the luminescence less strongly.

Additionally, lanthanides can be sensitized directly by nucleic acids. To this end, lanthanides must be bound to nucleic acids by some degrees of inner sphere coordination. For diffuse lanthanides or the ones with outer sphere coordination, luminescence is non-radiatively quenched by the first layer of water shell ${ }^{121}$. In a study by Feig et al. it was proposed that in a DNA sequence the keto tautomer of guanines can coordinate $\mathrm{Eu}^{3+}$ via $\mathrm{N}^{7}$ and $\mathrm{O}^{6}$ sites. The enol tautomer of guanines were proposed to be able to coordinate to both $\mathrm{Tb}^{3+}$ and $\mathrm{Eu}^{3+}$ via $\mathrm{N}^{1}$ and $\mathrm{O}^{6}$ sites. ${ }^{113}$

Sensitized luminescence of $\mathrm{Tb}^{3+}$ and $\mathrm{Eu}^{3+}$ have been used for detection of G-quadruplex forming DNAs ${ }^{122}{ }^{123}$. Sensitized luminescence of $\mathrm{Tb}^{3+}$ upon binding to G-quadruplex forming DNAs suggested that $\mathrm{Tb}^{3+}$ occupies the central cavity of G-tetrads of a quadruplex. The hypothesis was further proved by CD spectroscopy and FRET analysis of two covalently bound fluorophors upon $\mathrm{Tb}^{3+}$ titration ${ }^{124}$. Binding of lanthanide ions to biologically relevant RNAs e.g. RNNA $^{125}$ and hairpin ribozyme ${ }^{126}$ had great impact on understanding the properties of metal ion binding sites of these natural sequences. In addition to the direct sensitized luminescence studies, luminescence resonance energy transfer $(\mathrm{LRET})^{127}$ between a site-bound $\mathrm{Tb}^{3+}$ ion and a covalently attached Cy3 dye was employed to study 
natural systems such as U2-U6 snRNA complex. Based on LRET and in combination with biochemical and mutational studies three metal ion binding sites were proposed for U2-U6 snRNA complex, in consensus sequence ACAGAGA, AGC triad and internal stem loop (ISL) of U6 snRNA ${ }^{121}$. More recently the activity of G-quadruplex hemin deoxyribozyme was promoted upon presence of $\mathrm{Tb}^{3+}$ as a stabilizer of the G-quadruplex ${ }^{74}$.

The application of lanthanides in molecular biology is not limited to luminescence studies. $\mathrm{Tb}^{3+}$ footprinting assays have been used to study natural folding of RNA molecules such as tRNA ${ }^{128}$ or Hepatitis Delta Virus (HDV) ribozyme ${ }^{129}$. Micromolar concentration of $\mathrm{Tb}^{3+}$ can probe metal ion binding sites via site-specific cleavage while millimolar concentrations of lanthanides are normally used for $\mathrm{Tb}^{3+}$ footprinting assays based on the accessibility of the RNA backbone to the solution ${ }^{126}$. The ability of lanthanide ions for phosphodiester bond cleavage was shown to be enhanced when coordinated with synthetic complexes consistent of pyridyl, hydroxyl-benzyl and nitro functional groups ${ }^{130}$. Enhanced RNA strand scission of lanthanides complexes were further developed toward designing artificial sequence- specific ribonucleases ${ }^{131}$.

Lanthanides also have been used in biochemical assays for functional studies of ribozymes. For hammerhead ribozyme or the leadzyme a bell-shaped titration curve was observed upon titration of lanthanide in the presence of standard bivalent metal ions i.e. $\mathrm{La}^{3+}$ titration for $\mathrm{Mg}^{2+}$ dependent reaction of hammerhead ${ }^{132}$ and $\mathrm{Nd}^{3+}$ titration for $\mathrm{Pb}^{2+}$ dependent reaction of leadzyme ${ }^{133}$. These observations led to the hypothesis of a two-metal ion mechanism for catalysis of RNA cleavage ${ }^{134}$. Two-metal ion mechanism was proposed as a general mechanism for RNA cleaving activities of other catalytic RNAs e.g. RNase P hydrolysis of tRNA precursor or group I and group II intron self-splicing ${ }^{97}$. For two-metal ion mechanism, one of the metal ions activates the attacking water or sugar hydroxyl at the cleavage site, while the other metal ion coordinates and stabilizes the oxyanion leaving group. Both ions act as Lewis acids and stabilize the expected pentavalent transition state.

The RNA cleaving deoxyribozyme 8-17 was inhibited upon presence of micromolar concentrations of $\mathrm{Tb}^{3+} .{ }^{135}$ In contrast, lead dependent deoxyribozyme accepted $\mathrm{Lu}^{3+}, \mathrm{Tm}^{3+}$ and $\mathrm{Tb}^{3+}$ as cofactor for RNA cleavage catalysis ${ }^{111}$. The lead dependent deoxyribozyme was active in presence of $60 \mu \mathrm{M}$ of lanthanide and $10 \mathrm{mM} \mathrm{Mg}^{2+}$ when $\mathrm{Zn}^{2+}$ was absent. Recently, new deoxyribozymes were selected in vitro which strictly require lanthanides in addition with $\mathrm{Zn}^{2+}$.

Altogether, lanthanides have been proven to be useful as a probing agent for biophysical and biochemical studies of metal ion binding sites of (deoxy)ribozymes. The $\mathrm{Mg}^{2+}$ binding sites of several ribozymes have been investigated by lanthanides. 


\subsection{Solid phase synthesis of nucleic acids}

In parts of this thesis, combinatorial libraries of DNA/RNA chimera have been synthesized using the standard protocol for solid phase synthesis of RNA which is described in this section. Solid phase synthesis of RNA is usually performed on controlled-pore glass (CPG) or polystyrene solid supports. Phosphoramidite oligonucleotide synthesis proceeds in the 3'- to 5'-direction (opposite to the 5'- to 3'direction of DNA and RNA biosynthesis). RNA synthesis consists of iterated rounds of a cycle with four steps; 1) coupling of the new phosphoramidite to the immobilized growing RNA strand, 2) Capping 3) oxidation of the phosphorus atom in the backbone, and 3) detritylation and formation of a free 5'-OH of the growing RNA strands (Fig 1.11-1).

The building blocks of RNA synthesis (phosphoramidites) bear several orthogonal protection groups. The 5'-OH is protected by 4,4'-dimethoxytrityl (DMT). The 2' position bears a [(triisopropylsilyl)oxy]methyl (TOM) protection group. The phosphoramidite moiety bears a ßcyanoethyl protection group and contains a diisopropylamino group. Before each round, mild acidic conditions are used for removal of the 5'-DMT group of the growing strand. DMT cation has absorbtion at $498 \mathrm{~nm}^{136}$ which is usually monitored as the efficiency of the last coupling step. After removal of the DMT group and washing steps, the new phosphoramidite is incubated in presence of an activator and water-free acetonitrile. The activator is usually tetrazole or its derivative e.g. 5-Benzylthio-1H-tetrazole (BTT). 5'-hydroxyl group of the growing RNA strand attacks the phosphorus atom of the phosphoramidite moiety. Diisopropylamino group is released and a phosphite triester bond is formed (Fig 1.11-2) 


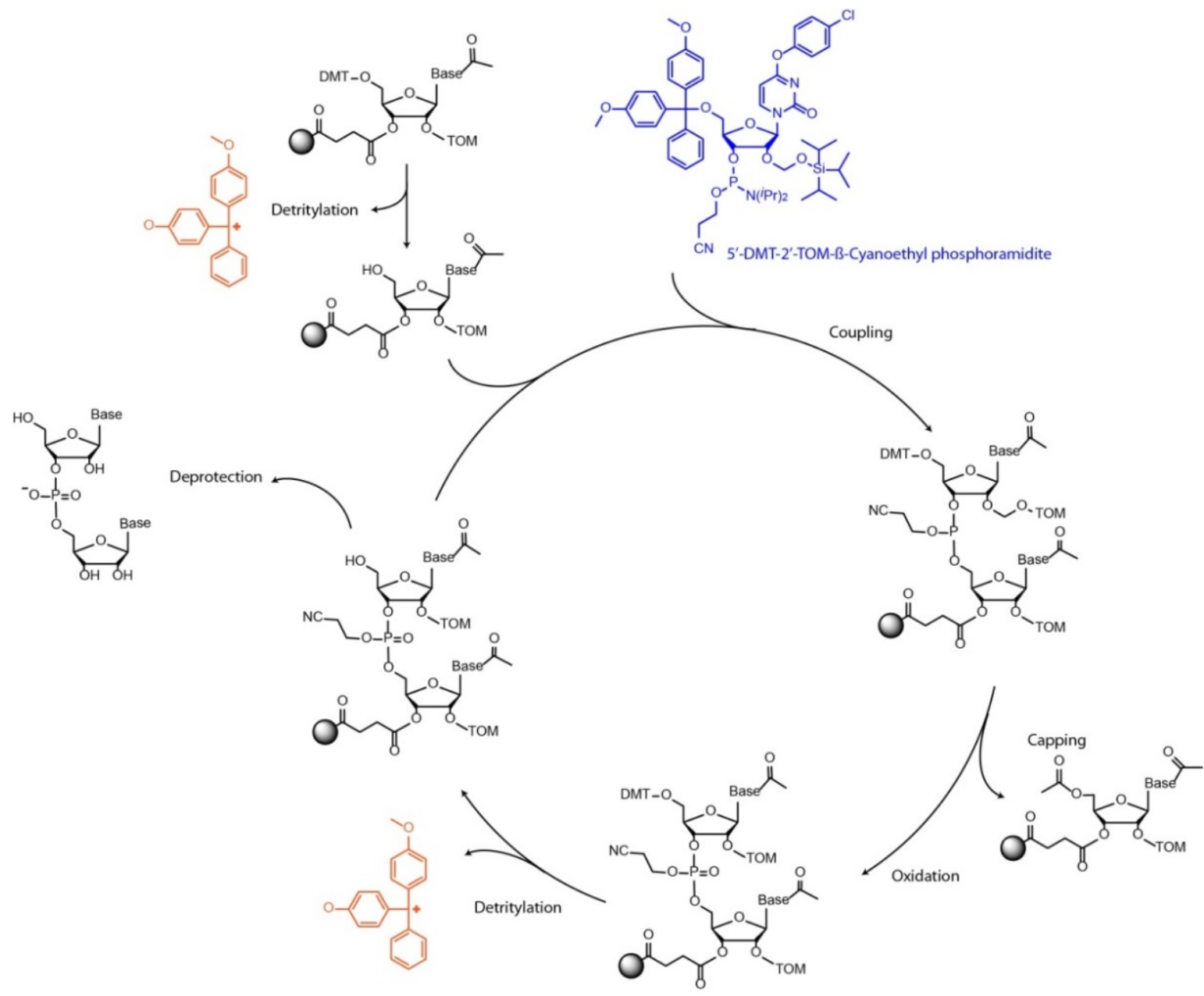

Fig 1.11-1 Solid phase synthesis of RNA. Synthesis starts with detritylation of the first phosphoramidite which is immobilized on solid support by an ester linkage. In each round, one phosphoramidite is coupled. The synthesis is continued by two capping steps before and after oxidation step. Final step of each round is deprotection of the 5'-DMT group. After final round, the synthesized RNA strands are cleaved off the support and get deprotected.

The unreacted strands are capped with an acetyl group in capping step using acetic anhydride. The phosphite triester formed in the coupling step is unstable to acid and must be converted to a stable pentavalent phosphate tri-ester prior to the next acidic detritylation step. Oxidation is performed in presence of iodine, water and pyridine. The phosphate group remains protected by the cyanoethyl group. The cyanoethyl group prevents undesirable reactions at phosphorus during subsequent synthesis cycles. Before the next coupling step, an additional capping step is necessary to remove any remained water molecule from oxidation step. The synthesis is continued by detritylation of the incorporated phosphoramidite.

After synthesis and final detritylation, one hour room temperature incubation with concentrated ammonium hydroxide efficiently hydrolyses the ester linkage of the synthesized strands and the solid support. The incubation is prolonged to $16 \mathrm{~h}$ at $55^{\circ} \mathrm{C}$ to deprotect the nucleobases from the acetyl and benzyl protection groups. The silyl moiety of the 2 ' protection group (TOM) is fluoride-labile ${ }^{137}$. The 2'-O-TOM protection group is removed by a fluoride solution e.g. tetrabutylamonium fluoride in tetrahydrofurane ${ }^{137}$. 
DNA synthesis is similar to RNA synthesis with slight differences. The 2' deoxy position does not require any protection group therefore DNA phosphoramidites are synthesized in fewer steps compared to RNA phosphoramidites ${ }^{138}$. The coupling efficiencies of DNA phosphoramidites are higher than RNA phosphoramidites due to less steric hindrance. The coupling times for DNA phosphoramidites are therefore shorter than RNA phosphoramidites. Finally, the last deprotection step of RNA (removal of TOM group) is not required for DNA oligos.
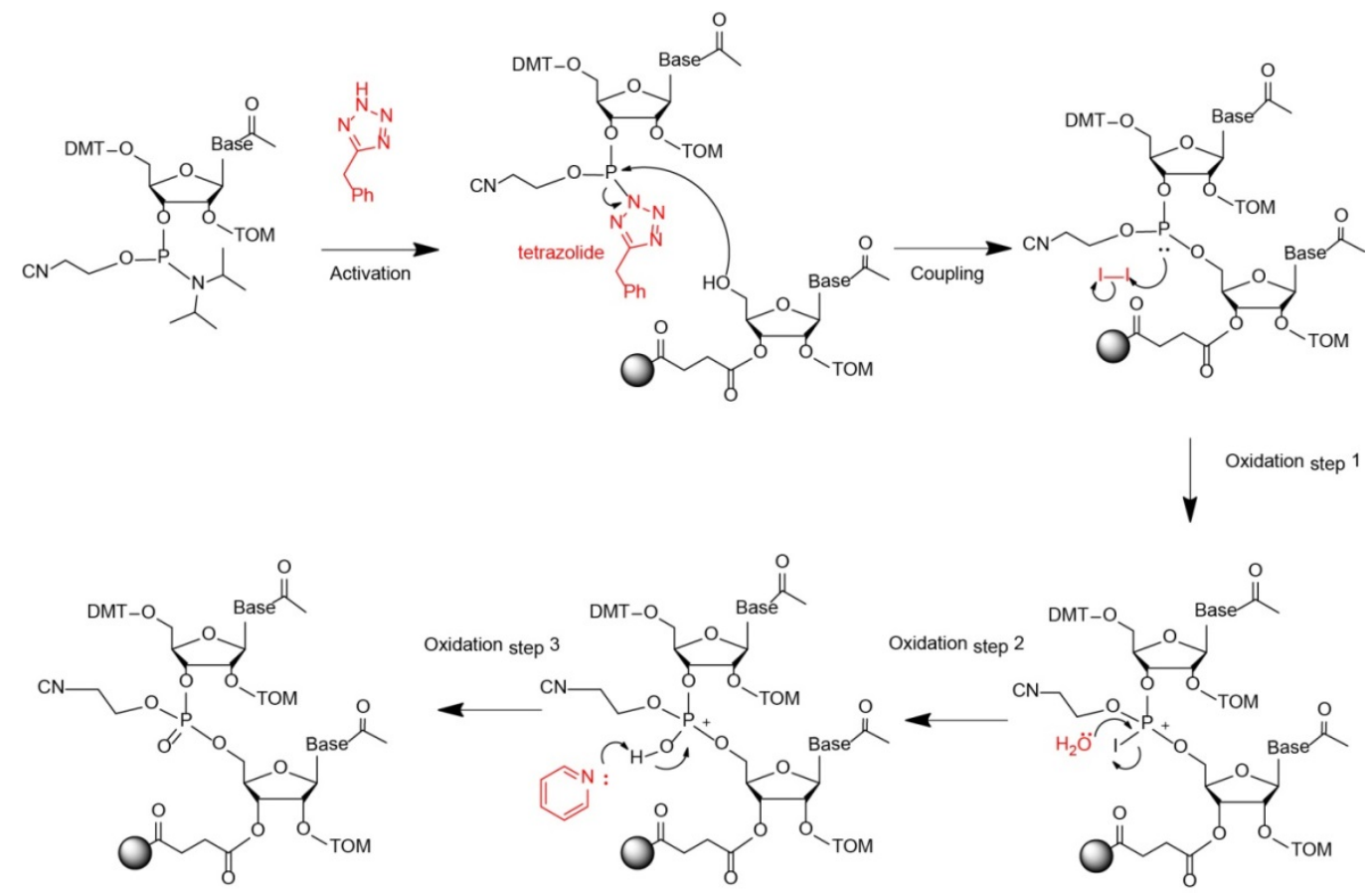

Fig 1.11-2 Activation, coupling and oxidation steps during RNA solid phase synthesis. In the first step, tetrazole or a tetrazole derivative protonates the nitrogen atom of the phosphoramidite moiety. After coupling and formation of a trivalent phosphite, the phosphorus atom is oxidized by iodine in presence of water and pyridine to a pentavalent phosphate. 


\subsection{Thesis outline}

Fundamental mechanistic study of deoxyribozymes is the main focus of this scientific report. Functional characterization of deoxyribozymes is necessary to expand their applications in molecular biology. The 2',5'-branched RNA forming deoxyribozymes which are a specific type of ligating deoxyribozymes were chosen for analysis and investigations. In this thesis, the research is presented in two main chapters.

Chapter 2 describes functional characterization of 2',5'-branched RNA forming deoxyribozymes. An efficient method for combinatorial mutation interference analysis (CoMA) is introduced to investigate nucleobase requirements of deoxyribozymes. Identifications of the essential nucleotides of 2',5'branched RNA forming deoxyribozymes is described in this chapter. The results of the CoMA were further validated using biochemical and biophysical approaches. The effects of lanthanide ions on the ligation rates of deoxyribozymes have been extensively studied. The kinetic studies in presence of lanthanide revealed exciting acceleration effect upon presence of lanthanides which was beneficially utilized for the application of deoxyribozymes.

The focus of chapter 3 is on application of RNA ligating deoxyribozymes. Preparative synthesis of 2',5'-branched RNAs was the first immediate application of this type of deoxyribozymes. $\mathrm{Tb}^{3+}$ mediated acceleration reduced the reaction times also in preparative scale. Experiments have been setup to build up platforms of using branched RNAs to answer questions of molecular biology. Preliminary data on RNA-RNA and RNA-protein interactions of components of yeast spliceosome in vitro with 2',5'-branched RNAs and in vitro translation using branched RNAs as the template are described shortly in chapter 3 . The binding assays have been in collaboration with the group of Dr. Vladimir Pena, Research Group Macromolecular Crystallography at Max Planck Institute for Biophysical Chemistry (MPI-BPC), Göttingen, Germany and the group of Professor Reinhard Lührmann, department of Cellular Biochemistry at MPI-BPC. The in vitro translation studies were in collaboration with the group of Professor Marina Rodnina, the department of Physical Biochemistry at MPI-BPC.

The main application described in chapter 3 is based on the benefits of $\mathrm{Tb}^{3+}$ for post-synthetic attachment of (labeled-) GTPs to RNA molecules by deoxyribozymes. Conditions for preparative GTP attachment to model RNA and biologically relevant RNAs in presence of $\mathrm{Tb}^{3+}$ have been optimized and reported. Additionally, strategies have been proposed for purification of (labeled-)GTP-attached long RNA molecules. The removal of non-GTP-attached RNAs is especially crucial when double labeling of a long RNA is aimed. The chapter 4 of the thesis is a general discussion on the characterization and application of deoxyribozymes. Material and methods are described in chapter 5. Chapter 6 includes lists of the oligonucleotides used in this thesis and list of abbreviations. 


\section{Functional characterization of deoxyribozymes}

At the time of start of this project, traditional approaches e.g. deletion or point mutations were available for characterization of functional DNAs e.g. deoxyribozymes. Analysis of single point mutants is laborious and does not provide comprehensive insights. We developed a new approach for combinatorial and reliable simultaneous investigation of individual nucleotides of functional DNA i.e. combinatorial mutation interference analysis (CoMA $)^{139}$. CoMA revealed functionally important nucleotides in two deoxyribozymes, 9DB1 $1^{31}$ and $7 \mathrm{~S} 11^{23}$ and led to engineer the enzyme 9DB1 to a minimized version. Knowing necessary nucleotides for catalysis, the next step was investigation of the functional groups of those necessary nucleotides. To this end, nucleotide analogue interference mapping of DNA (dNAIM) was developed in our lab $^{140}$. In this study CoMA and dNAIM have been used to investigate 2',5'-branched RNA forming deoxyribozymes. The two methods are described in the next section.

\subsection{Combinatorial mutation interference analysis (CoMA)}

CoMA consists of four steps, 1) combinatorial solid phase synthesis of mutant libraries, 2) separation of active mutants, 3) Site-specific cleavage at the mutation sites and 4) analysis of interference pattern by denaturing PAGE. The first step is preparation of mutant DNA libraries. In each library, the mutation sites are encoded by a 2'-hydroxyl group as a chemical tag. Solid phase synthesis of such DNA-RNA chimeras demands precautions and procedures of RNA synthesis.

Solid phase synthesis is an orthogonal chemical procedure which employs protected (deoxy) ribonucleoside phosphoramidites as building blocks. Single stranded nucleic acids are synthesized in 3' to 5' direction. CoMA employs standard procedure of RNA solid phase synthesis (described in section 1.10). The pohsphoramidite solutions used for CoMA are mixtures of 2'-O-TOM-protected ribonucleotide phosphoramidites $(\mathrm{rN})$ and deoxyribonucleotide phosphoramidites $(\mathrm{dN})$ rather than only $\mathrm{rN}$ phosphoramidites. For preparation of the mixtures, the $\mathrm{rN}$ and $\mathrm{dN}$ phosphoramidites are mixed in a ratio that statistically would result in one ribonucleotide mutation per DNA molecule for most of the synthesized strands. Four mutant libraries need to be synthesized i.e. one library for each rN mutation. Synthesis of each mutant library requires four mixtures i.e. four parent $\mathrm{dN}$ phosphoramidites individually mixed with one of the $\mathrm{rN}$ phosphoramidites. Therefore, in total 16 different mixtures need to be prepared.

To prepare a mutant library with maximum population of single mutants in a 40 nucleotide long catalytic core, the probability of $\mathrm{rN}$ incorporation at each independent event of phosphoramidite coupling must be $5 \%$. This value is dependent on the length of the DNA of interest and is easily calculated based on the binomial distribution. 


$$
P\left(i, n, r N_{e}\right)=\left(\begin{array}{c}
n \\
i
\end{array}\right)\left(r N_{e}\right)^{i}\left(1-r N_{e}\right)^{(n-i)}
$$

In the formula above, $i$ is the number of mutations per molecule (here preferred to be 1 ), $r N_{e}$ is the effective ribonucleotide incorporation rate, $n$ is the number of coupling events for which rN/dN mixtures are used (which is for deoxyribozymes catalytic core normally 40), and $\left(\begin{array}{l}n \\ i\end{array}\right)$ is the combinatorial function. The relative coupling efficiencies of ribonucleotides are normally less than deoxyribonucleotides. In practice, the $\mathrm{rN}$ : $\mathrm{dN}$ ratio of mixtures must be 10:90 to efficiently incorporate $5 \%$ ribonucleotide at each coupling step. This ratio was determined by experimental calibration curves for mixtures of each ribonucleotides in deoxyribonucleotides (supplementary information of reference $\left.^{139}\right)$.
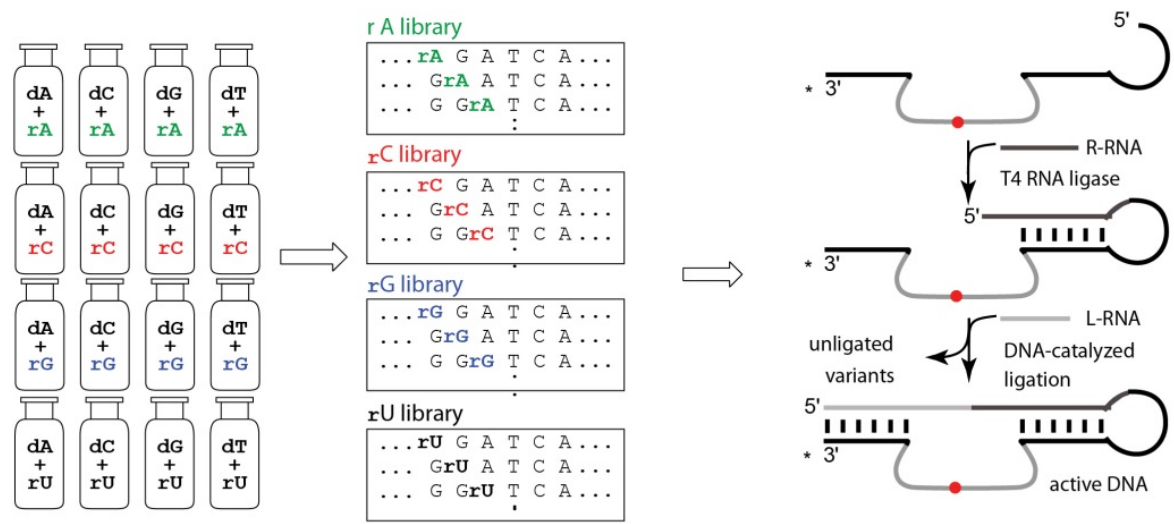

1) Combinatorial solid-phase synthesis

2) Separation of active mutants
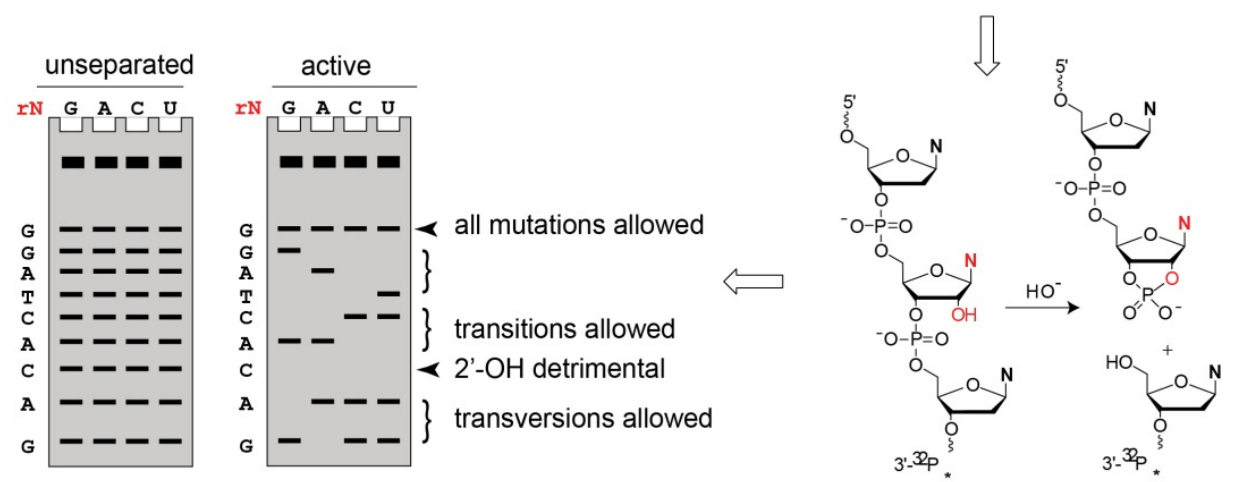

4) PAGE analysis of interference pattern

3) Alkaline hydrolysis

Fig 2.1-1 Combinatorial mutation interference analysis (CoMA). CoMA consists of four steps. The first step is statistic incorporation of 2'-OH tag encoded mutations within the functional DNA sequence. Afterwards, the active mutants are separated and the mutation sites are visualized upon site specific hydrolysis at mutation sites (step 3) and subsequent PAGE analysis using denaturing conditions (step 4).

The second step of CoMA is separation of active mutants. To this end, DNA mutant libraries are ligated to one of the RNA substrates by T4 RNA ligase. Providing the second substrate and the metal ions required for catalysis, the mutants in which the mutation is tolerated will catalyze the branching reaction of the two RNA strands. As one of the RNA strands is already ligated to the DNA library, the molecular 
weight of the active mutants is increased which allows separation of active mutants of the library from inactive ones.

The third step benefits from the 2'-OH tag which was introduced to the mutation sites by solid phase synthesis in the first step. The active and inactive fractions as well as unseparated libraries are radiolabeled at 3' end. Then, alkaline hydrolysis cleaves the DNA-RNA chimera backbone at the sites of RNA mutations and results in a ladder indicating the present mutations in each fraction. In the fourth step, the samples are loaded on a denaturing gel and band intensities are analyzed after quantification. After subtracting the background counts, interference values are defined for each position of the catalytic core and for each mutation by the ratio of the unselected band to the corresponding band in the active fraction.

$$
\text { Interference value }{ }^{\text {pos. } n}=\text { intensity }^{\text {pos. } n}{ }_{\text {unseparated library } / \text { intensity }}^{\text {pos. } n}{ }_{\text {active fraction }}
$$

Interference value of 1 means that the mutation had no effect on the reaction. We emphasize on interference values higher than 2 which means that the band intensity of the mutation at the active fraction was less than $50 \%$ of the band intensity at unselected library. Interference values higher than 10 are assigned as 10 since the error range increases by increase in the interference values.

CoMA is a powerful method to achieve comprehensive data about all three possible mutations of all 40 nucleotides of the deoxyribozymes catalytic core. Achieving the same data with traditional strategies i.e. synthesizing single point mutants and kinetic studies of individual mutants would be a lot more labor intensive and time consuming. However, CoMA is unable to reveal information in cases that the 2'-OH chemical tag itself is detrimental. Those positions are distinguishable in the PAGE analysis. In cases that a ribonucleotide with the parent nucleobases is not accepted (i.e. the hydrolyzed band is absent in the active fraction of parent library as well as all three mutant libraries), the 2'-OH is detrimental.

Nucleotide analogue interference mapping of DNA (dNAIM) ${ }^{140}$ has conceptual similarities to CoMA. dNAIM has four steps. In the first step libraries of modified nucleosides are synthesized. Modified nucleosides are distributed in the catalytic core and are tagged with a 2'-OH. Separation of the active modified DNAs, alkaline hydrolysis and PAGE separation is similarly performed as in CoMA. dNAIM supports complementary data on CoMA and pinpoints essential functional groups of essential nucleotides.

In this study, CoMA was applied to investigate nucleotide requirements of several 2',5' branch forming deoxyribozymes including 6BX22, 9F13 and 9F7. dNAIM was employed for further analysis of essential guanosines of 6BX22 core. 


\subsection{F7 deoxyribozyme family}

For chemical investigations of the catalytic mechanism of deoxyribozymes, we decided to study members of 9F7 deoxyribozyme family. 9F7 deoxyribozyme family was reported in 2003 by Silverman and coworker. They designed an in vitro selection experiment with the aim of achieving deoxyribozymes that catalyze the attack of a terminal 2'(3')-hydroxyl of an RNA onto a 5'-triphosphate of another RNA. They expected to select deoxyribozymes with linear 2'-5' or 3'-5' RNA products. The selection was performed with 40 nucleotide randomized region embedded in two binding arms for the RNA substrates. The acceptor RNA substrate contained 4 nucleotide long overhang $\underline{\text { UAUC. The final }}$ nucleotide was either A, C, dA or dC. dA and dC were used to promote 3'-5' linear ligation. Despite the original goal of that selection, all the active sequences catalyzed formation of branched RNAs using 2'$\mathrm{OH}$ of an internal nucleotide. The enzymes 9F13 and 9F18 used the 2'-OH of the first $\underline{\mathbf{U}}$ in the acceptor overhang to attack the alpha phosphate of the donor RNA whereas 9F21 and 9F7 employed the 2'-OH of the adjacent $\underline{\mathbf{A}}$ in the acceptor overhang. ${ }^{24}$

9F7 and 9F21 have been studied further for their substrate requirements. The result for 9F7 is summarized in Fig 2.2-1. For 9F7, the RNA overhang after the branch site adenosine could be removed completely. RNA ligation with no RNA overhang after the adenosine results in formation of a linear RNA with a 2'-5' junction. Formation of linear 2'-5' RNA by 9F7 was shown to be successful but the reaction rate was 100-fold slower compared to branched RNA formation. For fast ligation the overhang after the branch point needed to be at least one nucleotide long.

Although, selection rounds were performed in the presence of $\mathrm{Mg}^{2+}$, these enzymes showed faster reaction kinetics in the presence of $\mathrm{Mn}^{2+}$ e.g. for $9 \mathrm{~F} 7$ the $k_{\mathrm{obs}}$ was reported to be $\sim 0.3 \mathrm{~min}^{-1}$ with $20 \mathrm{mM}$ $\mathrm{Mn}^{2+}$ and $0.006 \mathrm{~min}^{-1}$ with $100 \mathrm{mM} \mathrm{Mg}{ }^{2+} \cdot{ }^{25}$ The two enzymes did not support any catalysis in the presence of $\mathrm{Zn}^{2+}, \mathrm{Ni}^{2+}$ or $\mathrm{Cd}^{2+}(10 \mu \mathrm{M}-10 \mathrm{mM}) .10 \mathrm{mM} \mathrm{Co}^{2+}$ resulted in a very low yield ligation with the $k_{\text {obs }}$ of $10^{5}$ fold slower than the reaction in the presence of $20 \mathrm{mM} \mathrm{Mn}^{2+}\left(0.000\right.$ '004 vs $\left.0.3 \mathrm{~min}^{-1}\right)$. The two enzymes showed no significant dependence on monovalent metal ions.

Originally, the enzyme 9F7 contained a deleted nucleotide in the left binding arm, resulting in a bulge loop in the acceptor RNA (five nucleotides upstream of the $\underline{\mathbf{A}}$ branch site). A complete base paired left binding arm of the enzyme showed to reduce the activity of the enzyme by 2-fold for $\mathrm{Mn}^{2+}$ dependent reaction and by 10 -fold for $\mathrm{Mg}^{2+}$ dependent reaction. Functional investigations by Silverman and coworker had used the complete base paired left hand binding arm. We used the "G-deletion” version of 9F7 as it was originally discovered and was shown to be faster.

The interaction of metal ions or deoxyribozyme's nucleobases with the functional groups of the substrate for the in-line attack of the 2'-OH to the alpha phosphate and release of the pyrophosphate is not well understood for these deoxyribozymes. Several methods are available to study metal ion 
interactions and to delineate critical nucleobase contacts in nucleic acid enzymes. Lanthanide ions have proven to be useful for probing metal ion binding in (deoxy) ribozymes. We focused on $\mathrm{Tb}^{3+}$ due to its particular properties for spectroscopic investigations. We performed combinatorial mutation interference analyses (CoMA) under various conditions to identify functional nucleotides of the 9F7 deoxyribozyme. The folding and interactions of 9F7 with metal ions were further investigated by luminescence and footprinting experiments.

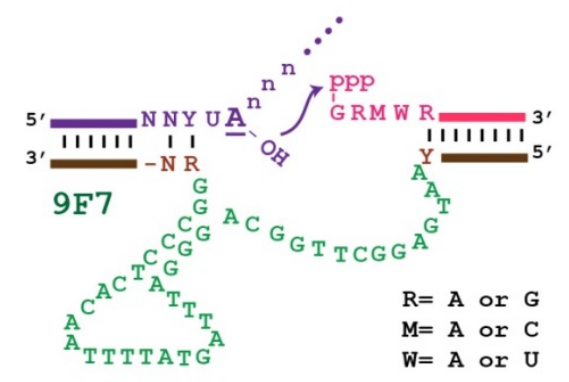

Fig 2.2-1 Original 9F7 and its substrate requirements. The branch site adenosine of the substrate is marked bold and underlined. 9F7 has 40 nucleotide in its single stranded catalytic core. Original report on the 9F7 had a point deletion in the left-binding arm of the enzyme. The enzyme was well studied according to its tolerance to substrate mutation. The substrate requirements of the 9F7 are marked in the figure. The figure is adapted from ${ }^{25}$.

\subsubsection{Lanthanide assisted acceleration of 9F7}

To study metal ion interactions of the 9F7 deoxyribozyme, we benefited from spectroscopic properties of rare earth elements i.e. lanthanides and in particular $\mathrm{Tb}^{3+}$. Firstly, we proved that the 9F7 deoxyribozyme interact competently with lanthanide ions. Impressive rate enhancement of 9F7 deoxyribozyme was observed upon addition of micromolar concentrations of $\mathrm{Tb}^{3+}$ when the reaction was performed in presence of millimolar concentrations of $\mathrm{M}^{2+}$. Slow $\mathrm{Mg}^{2+}$ dependent reaction of the enzyme ( $k_{\text {obs }} 0.003 \mathrm{~min}^{-1}$ in the presence of $80 \mathrm{mM} \mathrm{Mg}^{2+}$ ) was accelerated ca 120-fold upon addition of $\mathrm{Tb}^{3+}\left(k_{\mathrm{obs}} 0.35 \mathrm{~min}^{-1}\right.$ in the presence of $80 \mathrm{mM} \mathrm{Mg}^{2+}$ and $\left.100 \mu \mathrm{M} \mathrm{Tb}^{3+}\right)$. In a chase experiment, addition of $\mathrm{Tb}^{3+}$ one hour after start of the reaction had the same effect, indicating that the acceleration is particularly due to the presence of $\mathrm{Tb}^{3+}$ (Fig 2.2-2 A).

The $\mathrm{Tb}^{3+}$ mediated acceleration was dependent on $\mathrm{Tb}^{3+}$ concentration. Fig 2.2-2 $\mathrm{B}$ shows $\mathrm{Mg}^{2+}$ dependent catalysis of 9F7 in the presence of three representative concentrations of $\mathrm{Tb}^{3+}$. Addition of $\mathrm{Tb}^{3+}$ below a critical concentration had no significant effect. The reactions proceeded to completion in $>$ $8 \mathrm{~h}$ with the $k_{\mathrm{obs}}<0.01 \mathrm{~min}^{-1}$. At optimum $\mathrm{Tb}^{3+}$ concentration, the reaction was accelerated and reached $>80 \%$ yield in 5-10 min. Higher concentrations than the optimum resulted in fast reaction but low final yield.

Similar effects were observed by other lanthanides i.e. $\mathrm{La}^{3+}, \mathrm{Ce}^{3+}, \mathrm{Eu}^{3+}, \mathrm{Gd}^{3+}$ and $\mathrm{Yb}^{3+}$. Lanthanides were titrated to 9F7 reactions in the presence of $80 \mathrm{mM} \mathrm{Mg}^{2+}\left(\left[\mathrm{Ln}^{3+}\right]: 0-200 \mu \mathrm{M}\right)$. Plotting the yield of ligated RNA product as a function of lanthanide concentration revealed the optimum concentrations of lanthanides are in the same range. 

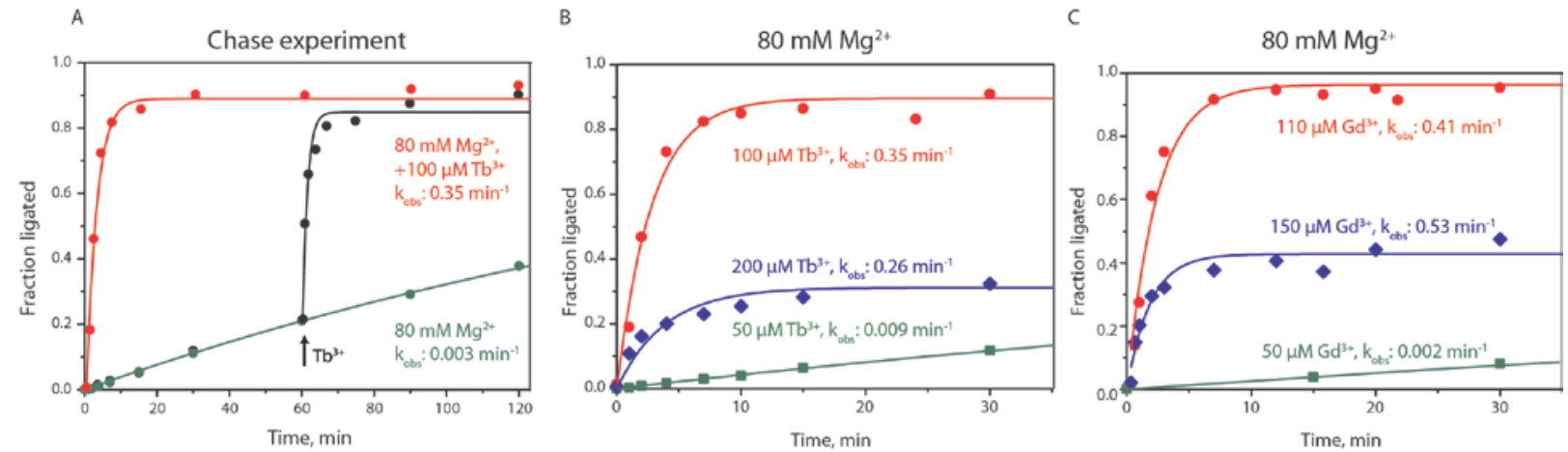

Fig 2.2-2 9F7 and the effect of $\mathrm{Tb}^{3+}$ on $\mathrm{Mg}^{2+}$ dependent reactions. A) Acceleration of 9F7 reaction. The $\boldsymbol{k}_{\mathrm{obs}}$ of $9 \mathrm{~F} 7$ reaction in the presence of $80 \mathrm{mM} \mathrm{Mg}{ }^{2+}$ is $0.003 \mathrm{~min}^{-1}$ (green). Presence of additional $100 \mu \mathrm{M} \mathrm{Tb} \mathrm{Tb}^{3+}$ increased the reaction rate to $0.35 \mathrm{~min}^{-1}$ (red). Addition of $\mathrm{Tb}^{3+}$ at $60 \mathrm{~min}$ after start of the reaction had the same effect (black). B) $\mathrm{Tb}^{3+}$ effect on 9F7 reaction is dependent on the concentration of $\mathrm{Tb}^{3+}$. $50 \mu \mathrm{M} \mathrm{Tb}^{3+}$ had no effect on the kinetic behavior of the enzyme and the reaction proceeded to reach $>80 \%$ yield after $8 \mathrm{~h} .100 \mu \mathrm{M} \mathrm{Tb^{3+ }}$ increased the reaction rate. The reaction yield reached $>80 \%$ within $10 \mathrm{~min} .200 \mu \mathrm{M} \mathrm{Tb}^{3+}$ increased the reaction rate but decreased the maximum yield. C) $\mathrm{Gd}^{3+}$ effect on $9 F 7$ reactions. The effect of $\mathrm{Gd}^{3+}$ was similar to $\mathrm{Tb}^{3+}$.

Addition of $\mathrm{Ln}^{3+}$ had no effect up to a "critical" concentration of ca. 50-70 $\mu \mathrm{M}$. The activity of the deoxyribozyme strongly increased up to the optimal concentration of 100-125 $\mu \mathrm{M} \mathrm{Ln}^{3+}$, at which $~ 80 \%$ yield was achieved within $5 \mathrm{~min}$. Further increase in $\left[\mathrm{Ln}^{3+}\right]$ up to $200 \mu \mathrm{M}$ caused reduced ligation yields. The maximum yield dropped significantly below those achievable in the absence of $\mathrm{Ln}^{3+}$ (Fig 2.2-3 for $\mathrm{Eu}^{3+}, \mathrm{Tb}^{3+}$ and $\mathrm{Gd}^{3+}$ titration and Fig 2.2-4 for $\mathrm{La}^{3+}$ and $\mathrm{Ce}^{3+}$ titration).
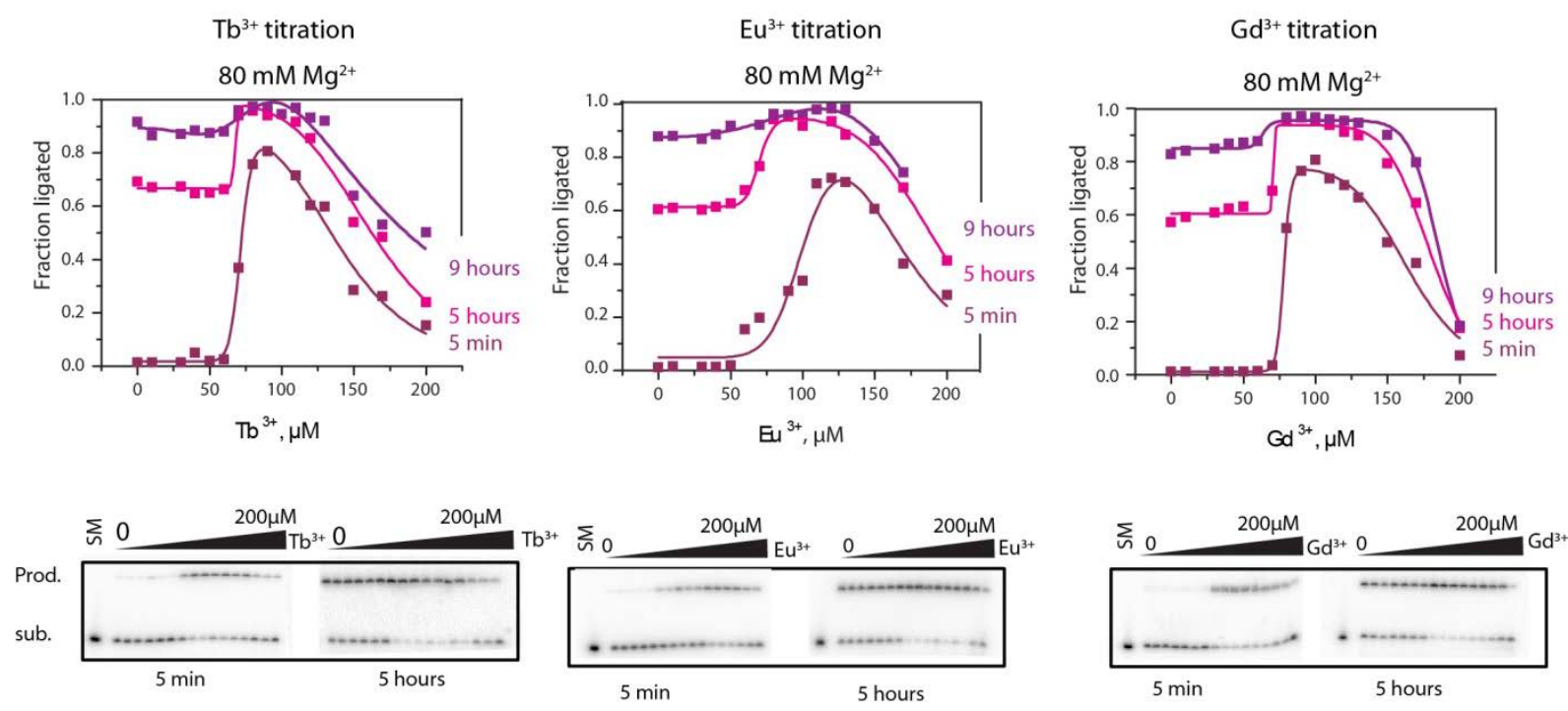

Fig 2.2-3 Titration kinetics of 9F7 in presence of $\mathrm{Mg}^{2+}$ for $\mathrm{Eu}^{3+}, \mathrm{Gd}^{3+}$ and $\mathrm{Tb}^{3+}$. Ligation yield of 9F7 in the presence of $80 \mathrm{mM} \mathrm{Mg}{ }^{2+}$ and increasing concentrations of the three lanthanides are plotted against time. The time points were taken at $5 \mathrm{~min}, 5 \mathrm{~h}$ and $9 \mathrm{~h}$. The curves were fitted to the equation: fraction ligated $=\left(\mathrm{Y}_{0}+\mathbf{Y}_{\mathrm{a}} *\left(\left[\operatorname{Ln}^{3+}\right]^{\mathrm{n}} /\left(\left[\mathrm{Ln}^{3+}\right]_{1 / 2, a}{ }^{\mathrm{n}}\right.\right.\right.$ $\left.\left.\left.+\left[\mathrm{Ln}^{3+}\right]^{\mathrm{n}}\right)\right)\right)^{*}\left(1-\left[\mathrm{Ln}^{3+}\right]^{\mathrm{m}} /\left(\left[\mathrm{Ln}^{3+}\right]_{1 / 2, \mathrm{~b}} \mathrm{~m}^{\mathrm{m}}+\left[\mathrm{Tb}^{3+}\right]^{\mathrm{m}}\right)\right)$. Fit values are summarized in table $2.2-1$. 

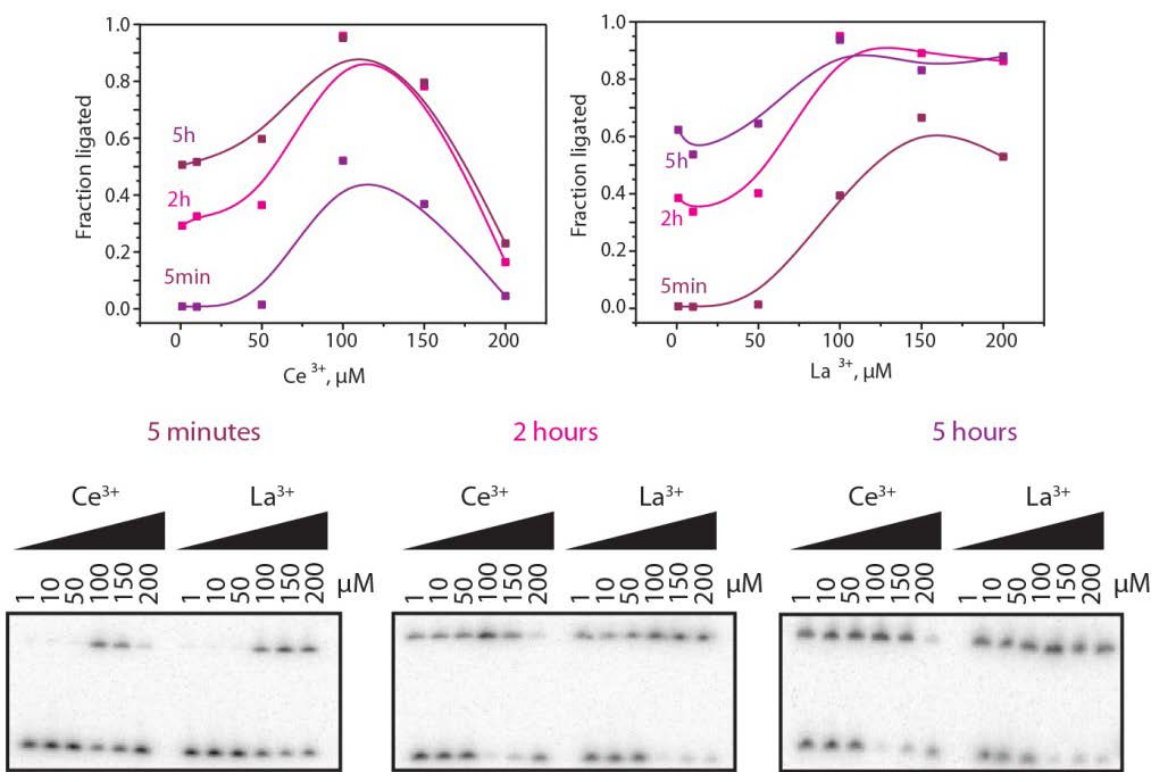

Fig 2.2-4 Titration kinetics of 9F7 in presence of $\mathrm{Mg}^{2+}$ for $\mathrm{Ce}^{3+}$ and $\mathrm{La}^{3+}$. Ligation yield of 9F7 in the presence of $80 \mathrm{mM}$ $\mathrm{Mg}^{2+}$ and increasing concentrations of lanthanides. The time points were taken at $5 \mathrm{~min}, 2 \mathrm{~h}$ and $5 \mathrm{~h}$. The connecting lines are B-spline interpolation of data (no fit values are available).

In the presence of $20 \mathrm{mM} \mathrm{Mn}{ }^{2+}$, the optimal $\left[\mathrm{Tb}^{3+}\right]$ was ca. 10 -fold lower than with $\mathrm{Mg}^{2+}$, which was best visible at early time points (below $2 \mathrm{~min}$ ). The optimum $\mathrm{Tb}^{3+}$ concentration was maintained below $50 \mu \mathrm{M}$ in the presence of $5 \mathrm{mM} \mathrm{Mn^{2+ }}$. The optimum range was broader at lower $\mathrm{Mn}^{2+}$ concentration (Fig 2.2-5).
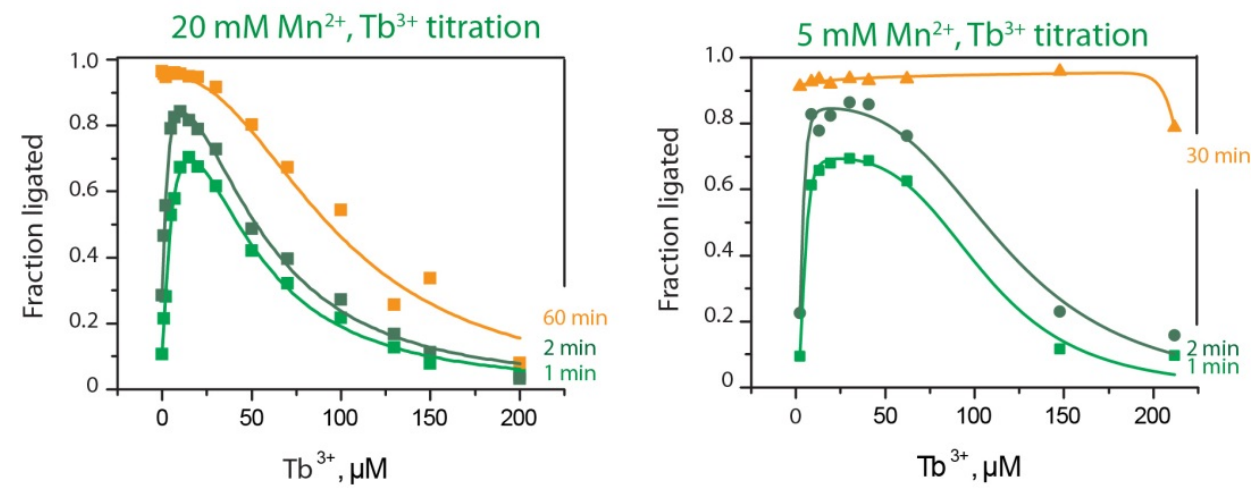

$20 \mathrm{mM} \mathrm{Mn}^{2+}, \mathrm{Tb}^{3+}$ titration

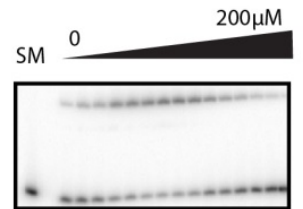

$1 \mathrm{~min}$

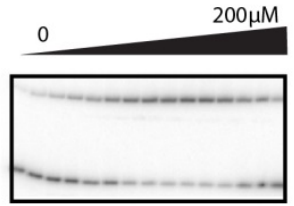

$2 \mathrm{~min}$

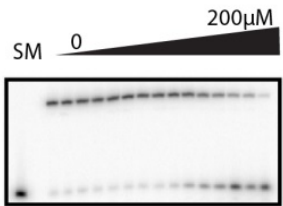

1 hour

Fig 2.2-5 $\mathrm{Tb}^{3+}$ titration kinetics of 9F7 in presence of $\mathrm{Mn}^{2+}$. Ligation yield of $9 \mathrm{~F} 7$ reaction in the presence of $20 \mathrm{or} 5 \mathrm{mM}$ $\mathrm{Mn}^{2+}$ and increasing concentrations of $\mathrm{Tb}^{3+}$. Gel images are only shown for $20 \mathrm{mM} \mathrm{Mn}{ }^{2+}$. 
Table 2.2-1 Fit values of the 9F7 activity and lanthanide titrations in the presence of $\mathrm{Mg}^{2+}$ or $\mathrm{Mn}^{2+}$. The ligation yields were plotted to the equation: fraction ligated $=\left(Y_{0}+Y_{a} *\left(\left[\operatorname{Ln}^{3+}\right]^{n} /\left(\left[\operatorname{Ln}^{3+}\right]_{1 / 2, a}{ }^{n}+\left[\operatorname{Ln}^{3+}\right]^{n}\right)\right)\right) *\left(1-\left[\operatorname{Ln}^{3+}\right]^{m} /\left(\left[\operatorname{Ln}^{3+}\right]_{1 / 2, b}{ }^{m}+\right.\right.$ $\left.\left.\left[\mathrm{Tb}^{3+}\right]^{\mathrm{m}}\right)\right)$.

\begin{tabular}{llllllll}
\hline Titration & reaction & $\mathrm{Y}_{0}$ & $\mathrm{Y}_{\mathrm{a}}$ & $\begin{array}{l}{\left[\mathrm{Ln}^{3+}\right]_{1 / 2, \mathrm{a}}} \\
\mathrm{\mu M}\end{array}$ & $\mathrm{n}$ & $\begin{array}{l}{\left[\mathrm{Ln}^{3+}\right]_{1 / 2, \mathrm{~b}}} \\
\mu \mathrm{M}\end{array}$ & $\mathrm{m}$ \\
\hline \multirow{2}{*}{$\mathrm{Tb}^{3+}$ with $80 \mathrm{mM} \mathrm{Mg}^{2+}$} & $5 \mathrm{~min}$ & 0.02 & 0.91 & 71 & 18 & 139 & 5 \\
& $5 \mathrm{~h}$ & 0.66 & 0.31 & 67 & 60 & 162 & 5 \\
$\mathrm{~Tb}^{3+}$ with $20 \mathrm{mM} \mathrm{Mn}^{2+}$ & $1 \mathrm{~min}$ & - & 0.9 & 4.6 & 1.3 & 50 & 1.8 \\
& $60 \mathrm{~min}$ & - & 0.95 & - & - & 97 & 2.2 \\
$\mathrm{Gd}^{3+}$ with $80 \mathrm{mM} \mathrm{Mg}^{2+}$ & $5 \mathrm{~min}$ & 0.01 & 0.76 & 77 & 32 & 164 & 8 \\
& $5 \mathrm{~h}$ & 0.60 & 0.33 & 70 & 118 & 178 & 11 \\
$\mathrm{Eu}^{3+}$ with $80 \mathrm{mM} \mathrm{Mg}^{2+}$ & $5 \mathrm{~min}$ & 0.008 & 0.76 & 81 & 7 & 171 & 5 \\
& $5 \mathrm{~h}$ & 0.61 & 0.33 & 69 & 14 & 192 & 7
\end{tabular}

As the optimal concentration of $\mathrm{Tb}^{3+}$ was shown to be only dependent of the type of bivalent metal ion, the kinetic activity of the enzyme was further investigated in the presence of increasing concentrations of $\mathrm{Mg}^{2+}$ and $\mathrm{Mn}^{2+}$ and in the presence or absence of optimum concentrations of $\mathrm{Tb}^{3+}$ i.e. $100 \mu \mathrm{M} \mathrm{Tb}^{3+}$ for $\mathrm{Mg}^{2+}$ dependent reactions and $10 \mu \mathrm{M} \mathrm{Tb}^{3+}$ for $\mathrm{Mn}^{2+}$ dependent reactions. Result showed that the presence of optimal concentrations of $\mathrm{Tb}^{3+}$ increases the affinity for the divalent metal ions (Fig 2.2-6). The $K_{\mathrm{d}, \text { app }}$ for $\mathrm{Mg}^{2+}$ decreased from $>200 \mathrm{mM}$ to $57 \mathrm{mM}$, and $K_{\mathrm{d} \text {,app }}$ for $\mathrm{Mn}^{2+}$ decreased ca. 3-fold from $7.6 \mathrm{mM}$ to $2.6 \mathrm{mM}$.
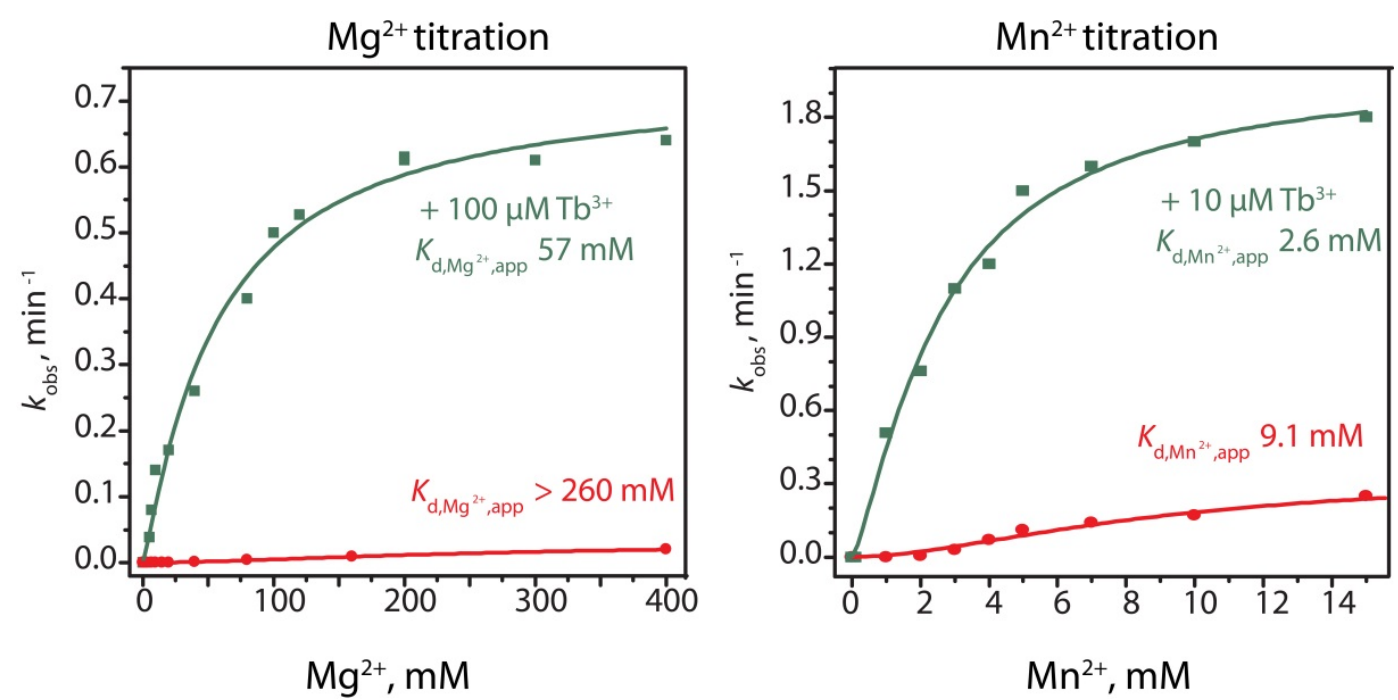

Fig 2.2-6 $\mathrm{Mg}^{2+}$ and $\mathrm{Mn}^{2+}$ titration kinetics of 9F7. Reaction rates of 9F7 in presence or absence of optimal concentrations of $\mathrm{Tb}^{3+}$ are plotted against $\left[\mathrm{Mg}^{2+}\right]$ or $\left[\mathrm{Mn}^{2+}\right]$. Optimum concentrations of $\mathrm{Tb}^{3+}$ in $\mathrm{Mn}^{2+}$ and $\mathrm{Mg}^{2+}$ dependent reactions were 10 and $100 \mu \mathrm{M}$ respectively. Titration curves were fitted to the equation $k_{\mathrm{obs}}=$ $k_{\max } *\left[\mathrm{M}^{2+}\right] /\left(K_{\mathrm{d}, \mathrm{app}}+\left[\mathrm{M}^{2+}\right]\right)$.

The impressive effect of $\mathrm{Tb}^{3+}$ was especially notable at low divalent metal ion concentrations. At $2 \mathrm{mM}$

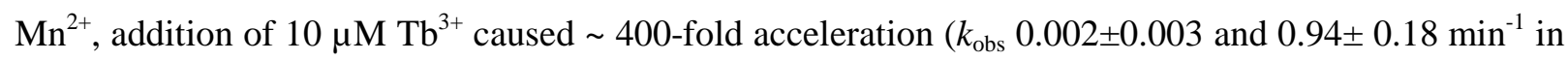
the absence and presence of $\mathrm{Tb}^{3+}$, respectively). For $\mathrm{Mg}^{2+}$ dependent reactions, the lowest $\mathrm{Mg}^{2+}$ 
concentration which led to detectable ligation product was $7 \mathrm{mM} \mathrm{Mg}^{2+}$. Addition of $100 \mu \mathrm{M} \mathrm{Tb}^{3+}$ caused $10^{4}$-fold faster ligation $\left(k_{\mathrm{obs}} 7 \times 10^{-6}\right.$ and $0.08 \mathrm{~min}^{-1}$ in the absence and presence of $\mathrm{Tb}^{3+}$, respectively). For the reaction in the presence of $7 \mathrm{mM} \mathrm{Mg}^{2+}$, in the absence of $\mathrm{Tb}^{3+}$, the ligation yield was only $1 \%$ after $24 \mathrm{~h}$. in the presence of $\mathrm{Tb}^{3+}$, the reaction yielded $>95 \%$ product in one hour, thereby $\mathrm{t}_{1 / 2}$ was reduced from $1600 \mathrm{~h}$ to $10 \mathrm{~min}$.

Table 2.2-2 Acceleration of $\mathrm{Mg}^{2+}$ dependent reactions of $9 \mathrm{F7}$ upon presence of $\mathrm{Tb}^{3+}$. $\boldsymbol{k}_{\mathrm{obs}}$ and $\mathrm{t}_{1 / 2}$ were calculated according to the fit equation: $Y=Y_{\max }\left(1-e^{-k_{o b s} \cdot t}\right), t_{1 / 2}=\ln 2 / k_{o b s}$.

\begin{tabular}{llllll}
\hline$\left[\mathrm{Mg}^{2+}\right]\left[\mathrm{Tb}^{3+}\right]$ & ligation & $k_{\text {obs }}$ & $\mathrm{t}_{1 / 2}$ & acceleration by $\mathrm{Tb}^{3+}$ \\
$\mathrm{mM}$ & $\mu \mathrm{M}$ & yield & $\mathrm{min}^{-1}$ & & \\
\hline 7 & - & $1 \%$ in $24 \mathrm{~h}$ & $7 \times 10^{-6}$ & $\sim 1600 \mathrm{~h}$ & \\
7 & 100 & $>95 \%$ in $1 \mathrm{~h}$ & 0.08 & $\sim 10 \mathrm{~min}$ & $\sim 10000$-fold \\
\hline 10 & - & $1 \%$ in $5.5 \mathrm{~h}$ & $3 \times 10^{-5}$ & $\sim 400 \mathrm{~h}$ & \\
10 & 100 & $>95 \%$ in $0.5 \mathrm{~h}$ & 0.14 & $\sim 5 \mathrm{~min}$ & $\sim 5000$-fold \\
\hline 80 & - & $>95 \%$ in $16 \mathrm{~h}$ & 0.003 & $\sim 4 \mathrm{~h}$ & \\
80 & 100 & $>95 \%$ in $0.15 \mathrm{~h}$ & 0.35 & $\sim 2$ min & $\sim 120$-fold \\
\hline
\end{tabular}

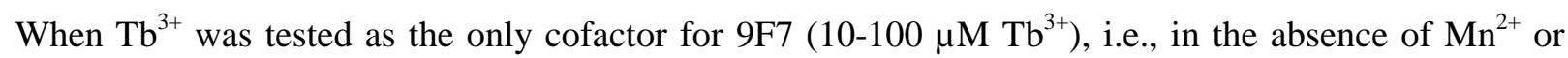
$\mathrm{Mg}^{2+}$, no ligation was observed up to 24 hours even when $3 \mathrm{M} \mathrm{NaCl}$ or $2 \mathrm{M} \mathrm{KCl}$ were present. In the presence of $80 \mathrm{mM} \mathrm{Mg}{ }^{2+}, \mathrm{Fe}^{3+}$ and $\mathrm{Al}^{3+}(1-100 \mu \mathrm{M})$ as trivalent non-lanthanide metal ions neither accelerated nor inhibited the $9 \mathrm{~F} 7$ reaction. Higher concentrations of $\mathrm{Fe}^{3+}$ and $\mathrm{Al}^{3+}$ caused degradation of the RNA substrates.

\subsubsection{HPLC analysis of the effect of $\mathrm{Tb}^{3+}$ on the reaction components}

The reduced ligation amplitude at high concentrations of lanthanides could potentially be rationalized by accelerated hydrolysis of the triphosphate at higher $\mathrm{Tb}^{3+}$ concentrations. This hypothesis was tested by HPLC analysis of the fate of donor RNA upon incubation under different metal ion combinations. Donor RNA (Tr12) was hybridized to a complementary DNA oligonucleotide (D064) resembling the deoxyribozyme binding arm. The retention times of the complementary DNA and tri- and monophosphorylated donor RNA are shown in Fig 2.2-7 A. The RNA-DNA complex was incubated under various conditions. More than $80 \%$ of triphosphate was still intact after $1 \mathrm{~h}$ in presence of $100 \mu \mathrm{M} \mathrm{Tb}^{3+}$ and $80 \mathrm{mM} \mathrm{Mg}^{2+}$. The triphosphate content decreased only slightly with $200 \mu \mathrm{M} \mathrm{Tb}^{3+}$ (Fig 2.2-7 B).

In 9F7 kinetic experiments the acceptor: DNA: donor ratio was 1:3:6. Scaled up reactions (50-fold) in had similar results compared to kinetic experiments when the reaction volume was increased 10-fold (100 $\mu$ l reaction). The ligation yield with $100 \mu \mathrm{M} \mathrm{Tb}^{3+}$ was $81 \%$ in 15 min while the ligation yield for $200 \mu \mathrm{M} \mathrm{Tb}^{3+}$ was only $30 \%$ after $1 \mathrm{~h}$. Ligation yield in presence of $80 \mathrm{mM} \mathrm{Mg}^{2+}$ (in the absence of $\mathrm{Tb}^{3+}$ ) was $68 \%$ in $8 \mathrm{~h}$ (Fig 2.2-8). 


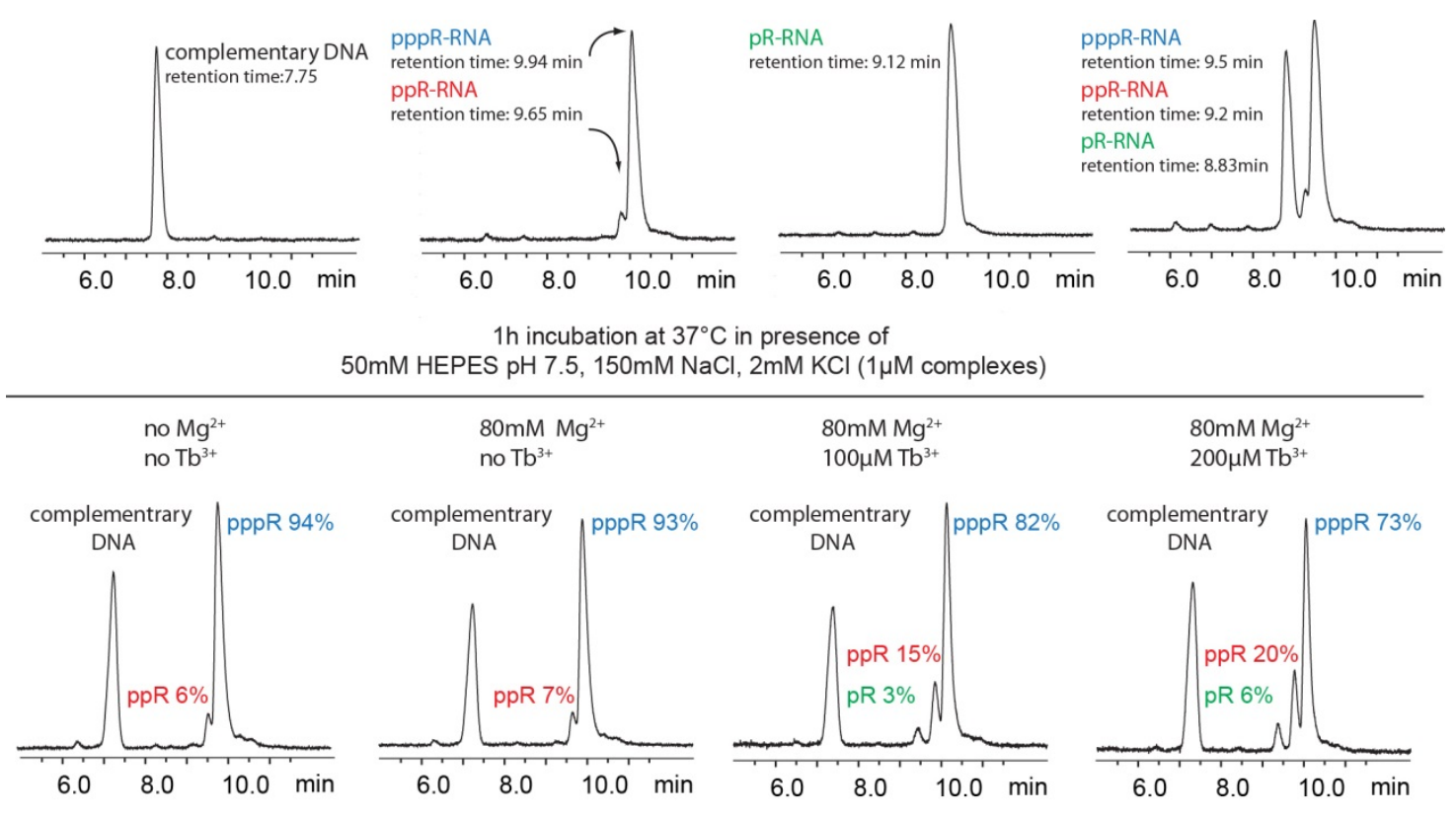

Fig 2.2-7 HPLC analysis of the triphosphorylated donor substrate. A) Validation of individual peaks of the HPLC chromatogram of the donor RNA. B) HPLC analyses of the fate of triphosphate donor RNA in presence of $\mathrm{Tb}^{3+}$. Donor RNA (Tr12) was hybridized to its complementary DNA (D064) to resemble the deoxyribozyme binding arm.

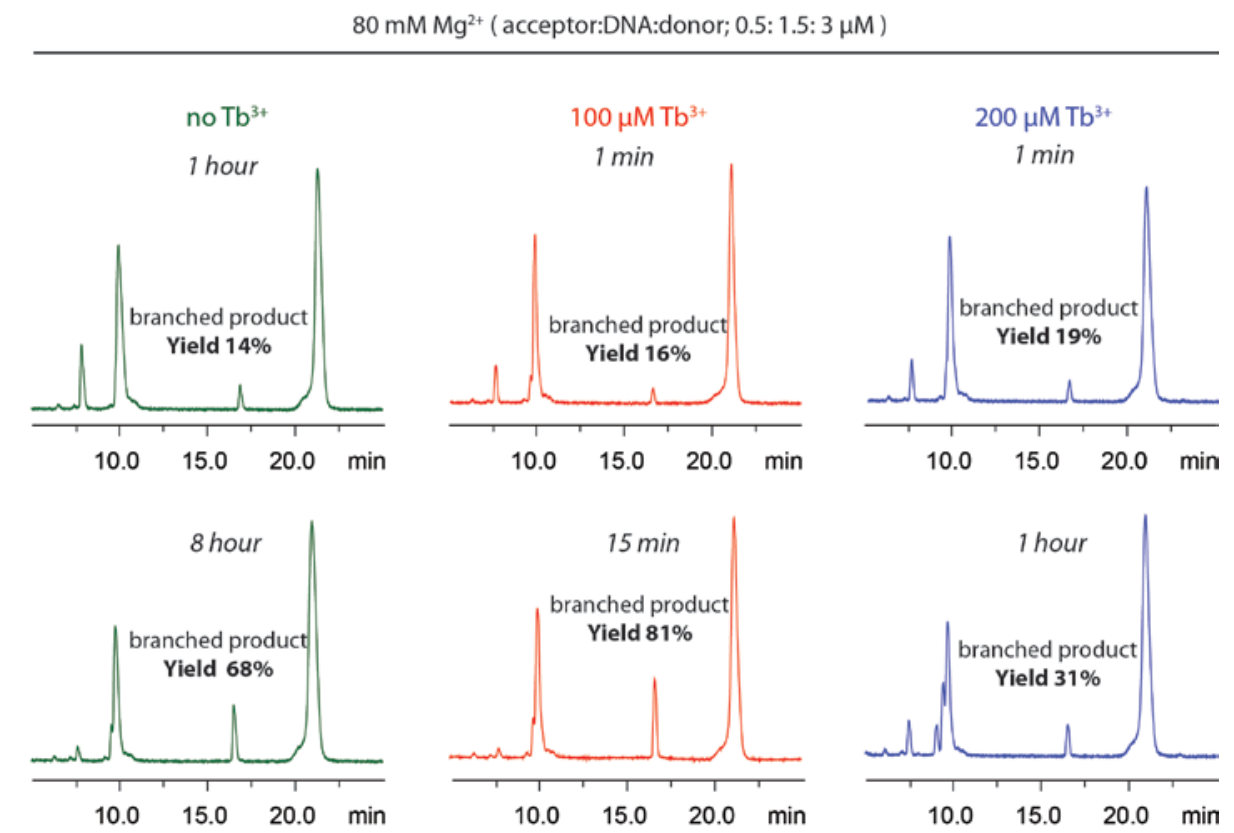

Fig 2.2-8 HPLC analyses of 9F7 reaction. The acceptor: DNA: donor RNA ratios were 50:150:300 pmol. The reactions have been performed in $100 \mu \mathrm{l}$ volume at $37^{\circ} \mathrm{C}$. Yields were calculated according to the equation: Yield $=\left(b r P / b r P_{\varepsilon}\right) /\left[\left(b r P / b r P_{\varepsilon}\right)+\left(a c . / a c c_{\varepsilon}\right)\right]$, when brP and ac. are the intensities under the $U V^{260 n m}$ trace for the branched product and acceptor RNA substrates and $\varepsilon$ is their extinction coefficient.

When the donor, acceptor, and deoxyribozyme were used in equimolar ratios for preparative purposes, the DNA-catalyzed RNA ligation reaction was also accelerated by $\mathrm{Tb}^{3+}$. To maintain good yield after short incubation times, the substrate-DNA concentration was $1 \mu \mathrm{M}$, resulting in 100 equivalents of $\mathrm{Tb}^{3+}$ in respect to pre-ligation complex. At higher substrate-DNA concentrations (lower $\mathrm{Tb}^{3+}$ to nucleic acid 
ratio), the ligation reaction was slower, likely due to increased unspecific binding of $\mathrm{Tb}^{3+}$ to the nucleic acids, causing reduced availability of “active” $\mathrm{Tb}^{3+}$ ions (Fig 2.2-9).

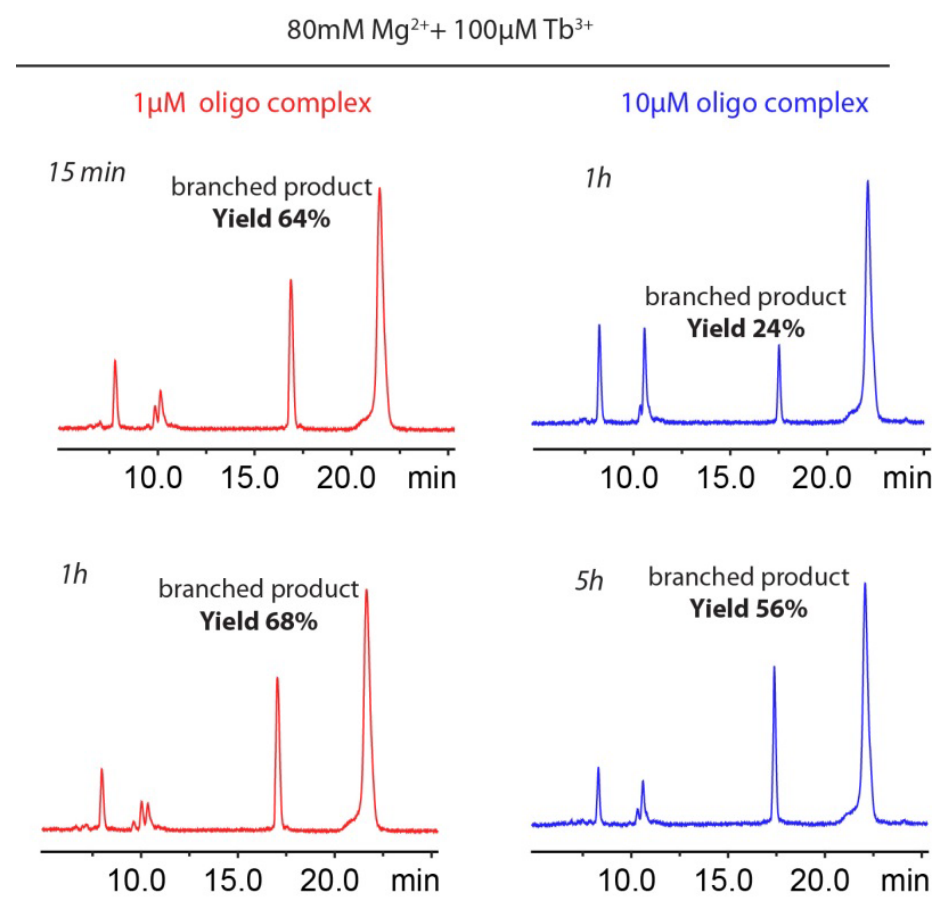

Fig 2.2-9 HPLC analyses of preparative 9F7 reactions. Acceptor: DNA: donor ratios are 1:1:1. The reactions were performed with $100 \mathrm{pmol}$ of each component in either 100 or $10 \mu \mathrm{l}$. The concentrations of the metal ions were constantly $80 \mathrm{mM} \mathrm{Mg}^{2+}$ plus $100 \mu \mathrm{M} \mathrm{Tb}^{3+}$, therefore the $\left[\mathrm{Tb}^{3+}\right] /[$ oligonucleotide complex] were 100 and 10 fold respectively for 100 and $10 \mu \mathrm{l}$ reaction volumes.

In summary the HPLC analysis of the nucleic acid components of the 9F7 reaction revealed that although decomposition of the triphosphorylated donor RNA to mono and dephosphorylated state is increased upon long incubation with $200 \mu \mathrm{M} \mathrm{Tb}^{3+}(>1 \mathrm{~h}$ ) but the extent of decomposition is much less than the inhibitory effects at high $\mathrm{Tb}^{3+}$ concentrations. Additionally, HPLC analysis showed that for preparative applications of $\mathrm{Tb}^{3+}$ acceleration, the ratio of $\mathrm{Tb}^{3+}$ metal ion to the RNA-DNA complexes should be maintained higher than 10 for fast ligation.

Another possible hypothesis for reduced ligation yield at high $\mathrm{Tb}^{3+}$ concentrations could be that $\mathrm{Tb}^{3+}$ promotes the reverse reaction. To test this hypothesis branched RNA product of the 9F7 was 5' radiolabeled and annealed with 9F7. Samples were incubated in the presence of increasing concentrations of $\mathrm{Tb}^{3+}$ and in the presence of bivalent metal ions and absence or presence of 5-50 $\mu \mathrm{M}$ inorganic pyrophosphate (PPi). The hypothesis was rejected as no specific hydrolysis of the branched product was observed up to $48 \mathrm{~h}$. These data also proved stability of the branched product at various $\mathrm{Tb}^{3+}$ concentrations. Fig 2.2-10 shows the results in the presence of $5 \mu \mathrm{M}$ PPi (equimolar to branched product). 


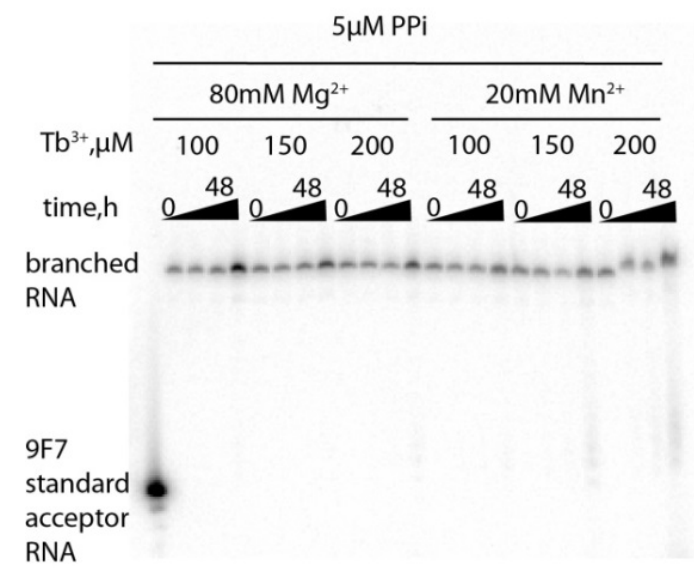

Fig 2.2-10 Stability of branched RNA product of 9F7. Incubation of branched RNA product of 9F7 with bivalent metal ions and high concentrations of $\mathrm{Tb}^{3+}$ in the presence of inorganic pyrophosphate (PPi).

In summary, functional characterizations of 9F7 confirmed necessity of the bivalent metal ions i.e. $\mathrm{Mn}^{2+}$ or $\mathrm{Mg}^{2+}$ for the catalysis. $\mathrm{Tb}^{3+}$ and other rare earth elements accelerated the reaction rate of the enzyme when provided at an optimal concentration. At higher concentrations the ligation reactions of 9F7 were fast but the maximum yields were reduced. HPLC analysis of the fate of the RNA substrates excluded possibility of the degradation of the RNA substrates or decompositions of the triphosphate of the donor. In addition, we did not observe promotion of the reverse reaction at high $\mathrm{Tb}^{3+}$ concentrations. We continued further studies on 9F7 by performing CoMA in presence of different metal ions.

\subsubsection{Combinatorial mutation interference analysis of 9F7}

Combinatorial mutation interference analysis (CoMA) provides comprehensive data about the nucleobase requirement of deoxyribozymes for catalysis in different conditions e.g. metal ions, $\mathrm{pH}$, time and temperature. In the case of 9F7, different nucleotides might be involved in formation of the catalytic core for $\mathrm{Mg}^{2+}$ and $\mathrm{Mn}^{2+}$ dependent reactions of 9F7. It is also conceivable that optimal concentrations of $\mathrm{Tb}^{3+}$ alter the nucleobase requirements of the enzyme. To compare necessary nucleotides of 9F7 at low and high bivalent metal ion concentrations, we decided to perform the separation step of CoMA at 2 $\mathrm{mM}$ and $20 \mathrm{mM} \mathrm{Mn}^{2+}$. To compare the effect of different metal ions we additionally performed the separation step of CoMA in the presence of $80 \mathrm{mM} \mathrm{Mg}{ }^{2+}$. To examine the influence of $\mathrm{Tb}^{3+}$, we included the CoMA in the presence of optimal concentrations of $\mathrm{Tb}^{3+}\left(10 \mu \mathrm{M} \mathrm{Tb}^{3+}\right.$ with $2 \mathrm{mM} \mathrm{Mn}^{2+}$ and $100 \mu \mathrm{M} \mathrm{Tb}^{3+}$ with $80 \mathrm{mM} \mathrm{Mg}^{2+}$ ). 


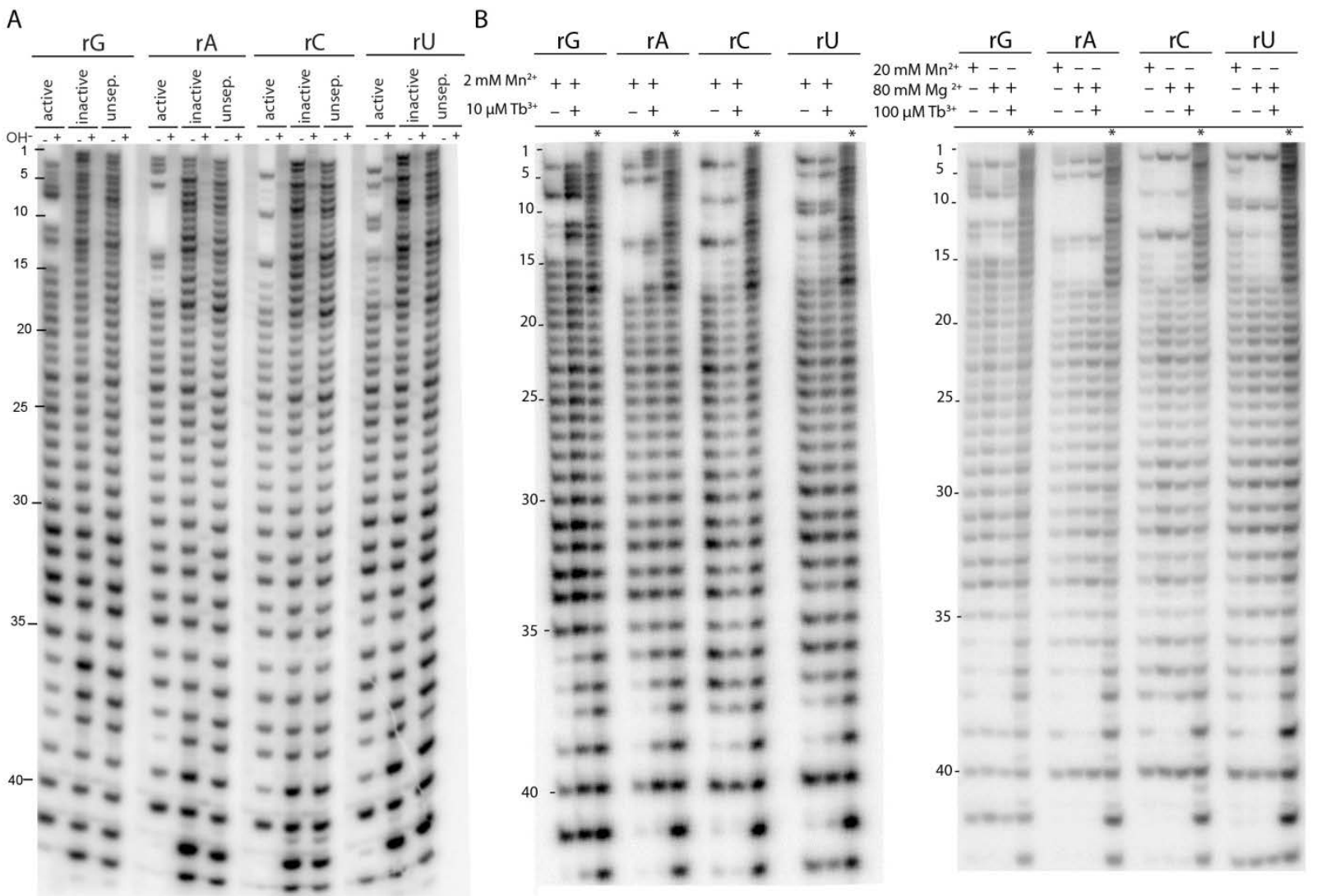

Fig 2.2-11 Autoradiogram of denaturing gels reflecting hydrolysis pattern of 9F7 combinatorial mutation interference analysis. CoMA was performed in 5 different conditions. Four mutant libraries were subjected for separation of active

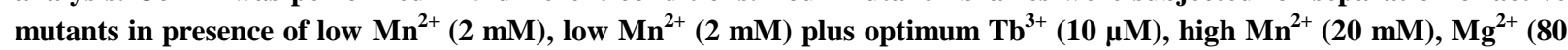
$\mathrm{mM})$ and $\mathrm{Mg}^{2+}(80 \mathrm{mM})$ plus optimum $\mathrm{Tb}^{3+}(100 \mu \mathrm{M})$. A) The gel for high $\mathrm{Mn}^{2+} \mathrm{CoMA}$ is shown as a representative gel. The separation of active mutant was performed in the presence of $150 \mathrm{mM} \mathrm{NaCl}, 2 \mathrm{mM} \mathrm{KCl}$ and $20 \mathrm{mM} \mathrm{MnCl}_{2}$ at $\mathbf{p H}$ 7.5. Active, inactive fractions and unselected libraries were hydrolyzed in presence of $10 \mathrm{mM} \mathrm{NaOH}$ for $10 \mathrm{~min}$ at $95^{\circ} \mathrm{C}$. For each lane a control water lane is loaded when the samples were incubated for $10 \mathrm{~min}$ at $95^{\circ} \mathrm{C}$ in the absence of

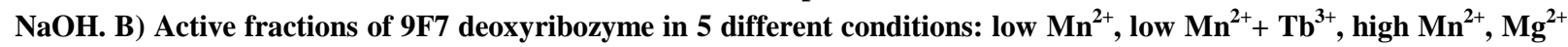
and $\mathrm{Mg}^{2+}+\mathrm{Tb}^{3+}$. Unselected libraries are loaded on the side as control. The upper parts of the gels are zoomed in in Fig 2.2-12.

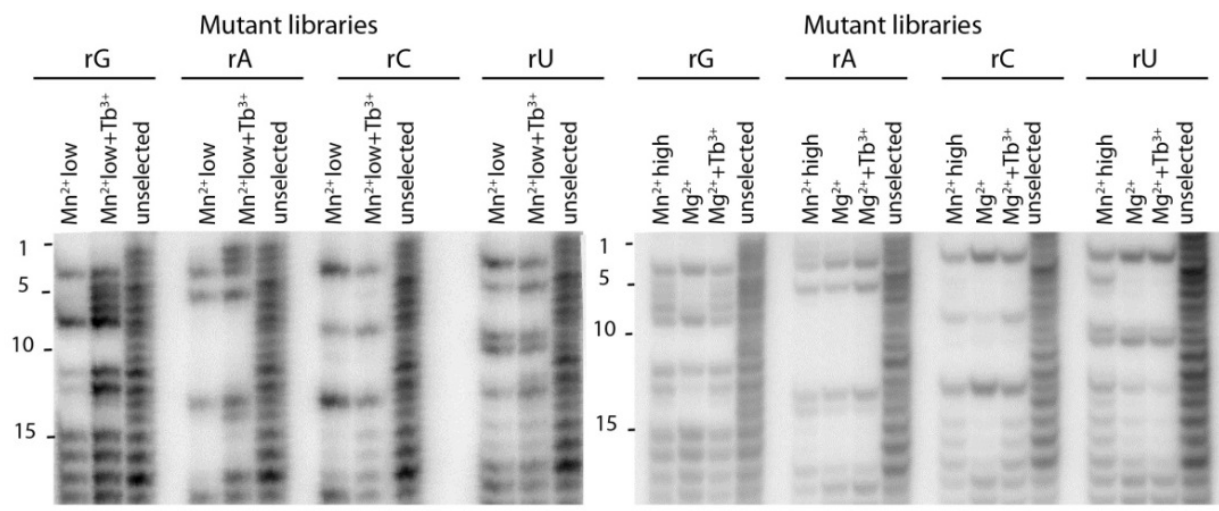

Fig 2.2-12 Autoradiogram of denaturing PAGE reflecting hydrolysis pattern of 9F7 active mutants at the 5' end of the catalytic core under 5 different conditions i.e. in presence of low $\mathrm{Mn}^{2+}(2 \mathrm{mM})$, low $\mathrm{Mn}^{2+}(2 \mathrm{mM})$ plus optimum $\mathrm{Tb}^{3+}(10$ $\mu M)$, high $\mathrm{Mn}^{2+}(20 \mathrm{mM}), \mathrm{Mg}^{2+}(80 \mathrm{mM})$ and $\mathrm{Mg}^{2+}(80 \mathrm{mM})$ plus optimum $\mathrm{Tb}^{3+}(100 \mu \mathrm{M})$. The hydrolysis was performed in presence of $10 \mathrm{mM} \mathrm{NaOH}$. This figure is a zoomed view of the top of the gels that are shown in Fig 2..2-11 B. 


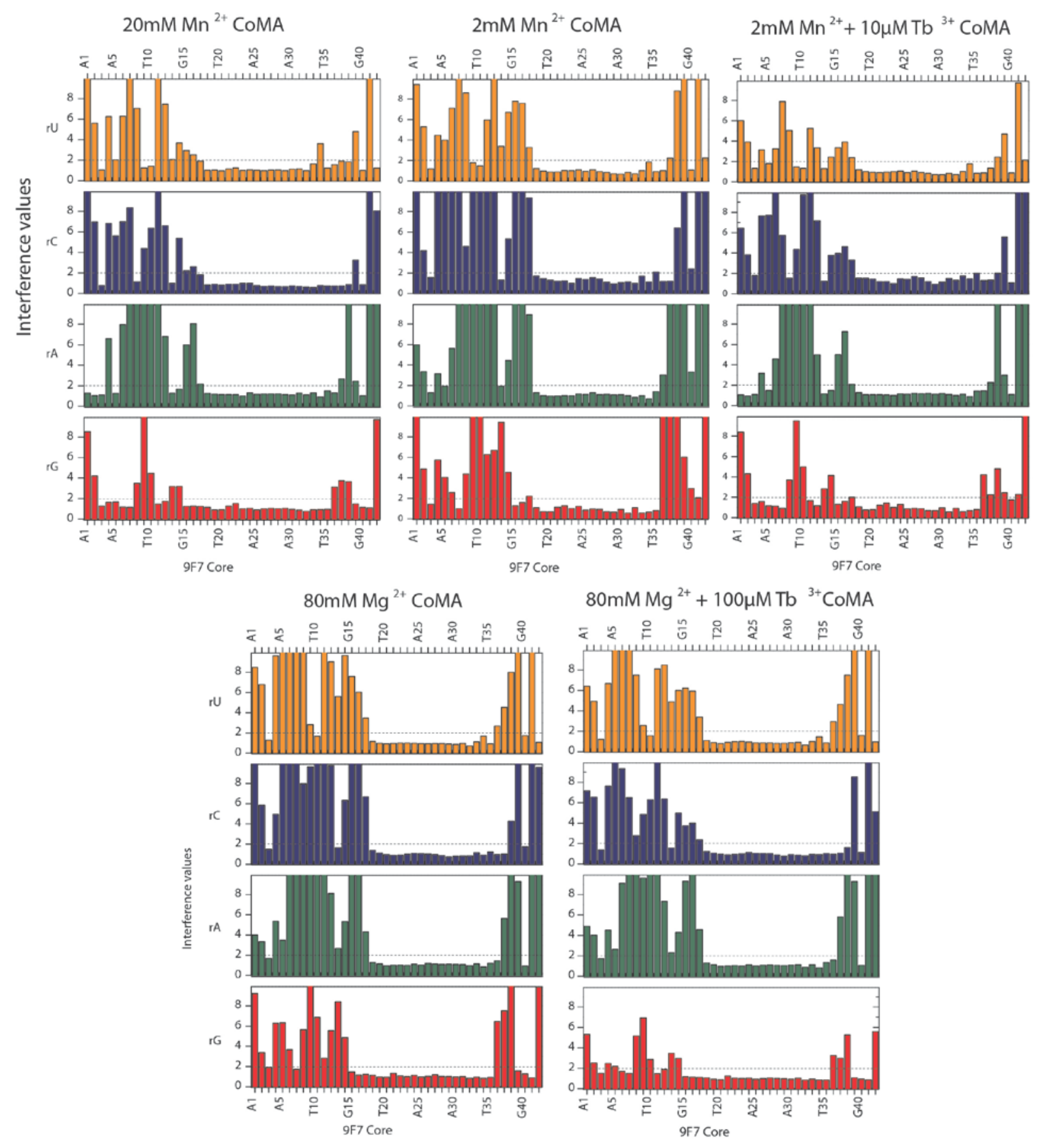

Fig 2.2-13 Interference values for 9F7 mutant libraries at 5 different conditions shown as bargraphs. Interference values were calculated based on the quantifications of the intensities of the bands of gel images in 2.2-11. Interference value for each position is defined as: Interference value ${ }^{\text {pos. } n}=$ intensity $^{\text {pos. } n}{ }_{\text {unseparated library }} /$ intensity $^{\text {pos. } n}$ active fraction . The interference values below 2.0 were considered as negligible. The interference values higher than 10.0 were assigned as 10.0. The interference values are grouped based on the mutations types. The $x$-axes of all plots are the sequence of the catalytic core of the enzyme in the direction of 5' to 3'. The conditions at which the 9F7 ligation reactions were performed are marked on top of each set of plots.

The ligation reaction time at separation step of CoMA affects presence of non-efficient mutants in the active fraction. For each of the 5 conditions, the 9F7 reaction times were selected according to the kinetic of the enzyme in the presence of individual conditions. The time in which the three-molecular format kinetics of the enzyme reached more than $90 \%$ of the maximum yield was chosen to stop the 9F7 
reactions for that specific metal ion condition. The reaction times were $10 \mathrm{~h}$ with $2 \mathrm{mM} \mathrm{Mn^{2+ }}, 8 \mathrm{~h}$ with $80 \mathrm{mM} \mathrm{Mg}^{2+}$, 2 h with $100 \mu \mathrm{M} \mathrm{Tb}^{3+}+80 \mathrm{mM} \mathrm{Mg}^{2+}, 1$ h with $10 \mu \mathrm{M} \mathrm{Tb}^{3+}+2 \mathrm{mM} \mathrm{Mn}^{2+}$, and $20 \mathrm{~min}$ with $20 \mathrm{mM} \mathrm{Mn}^{2+}$. For all five reactions, these incubation times reproducibly resulted in formation of ca $30 \%$ ligation product in all four mutant libraries. After separation and alkaline hydrolysis the samples were loaded on denaturing PAGE. Fig 2.2-11 A shows a representative gel for full set of CoMA. Fig 2.2-11 B shows the active fractions of the 5 tested conditions. Fig 2.2-12 is a zoomed in view of the active fractions in the 5' end of the catalytic core.

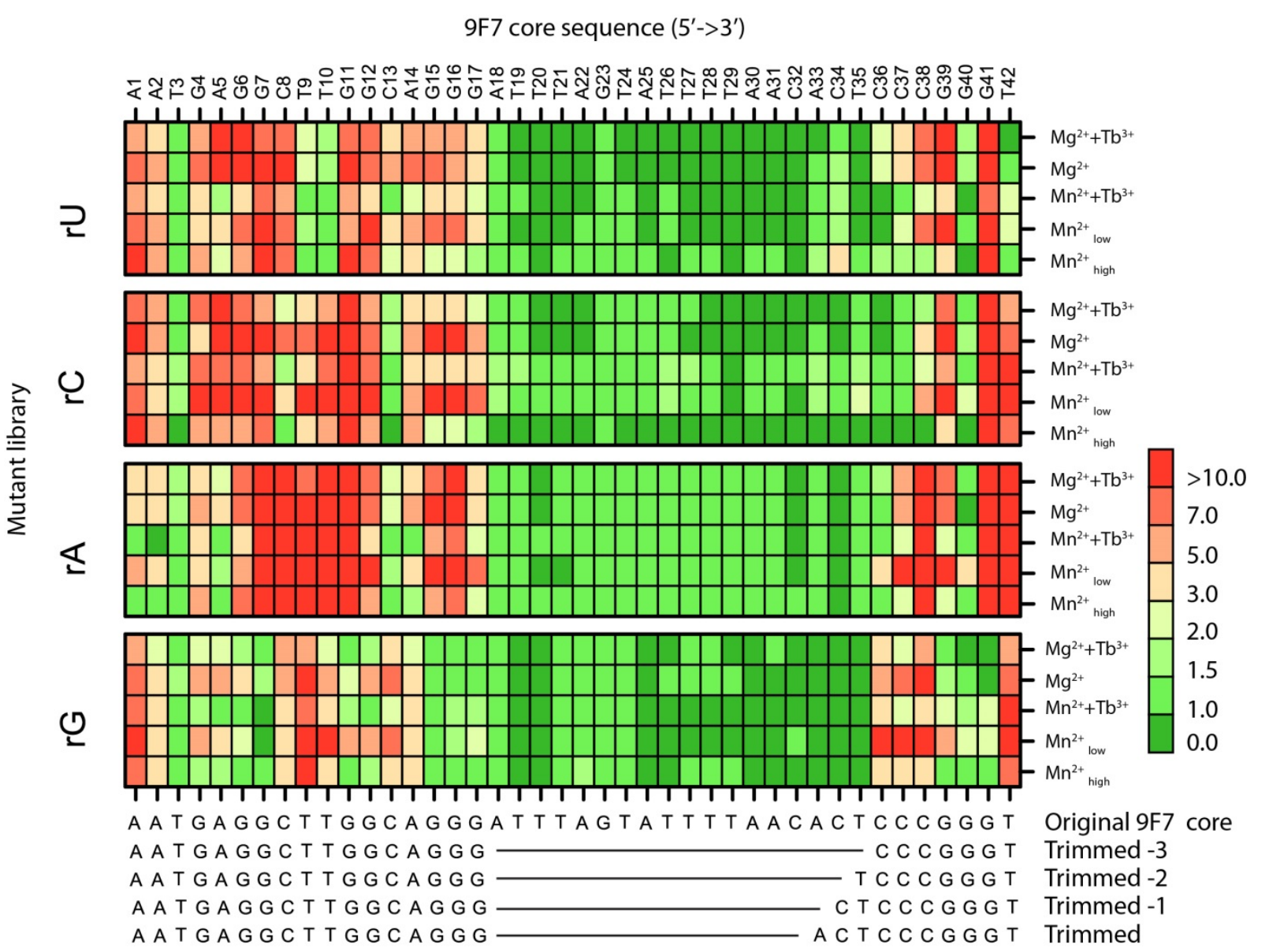

Fig 2.2-14 Contour graph of interference values of $9 \mathrm{F7}$ combinatorial libraries for the 5 tested conditions i.e. low $\mathrm{Mn}^{2+}$ (2 mM), low $\mathrm{Mn}^{2+}(2 \mathrm{mM})$ plus optimum $\mathrm{Tb}^{3+}(10 \mu \mathrm{M})$, high $\mathrm{Mn}^{2+}(20 \mathrm{mM}), \mathrm{Mg}^{2+}(80 \mathrm{mM})$ and $\mathrm{Mg}^{2+}(80 \mathrm{mM}) \mathrm{plus}^{2}$ optimum $\mathrm{Tb}^{3+}(100 \mu \mathrm{M})$. The high interference values which reflect detrimental effects of a mutation are assigned with red colors. Low interference values which are refection of tolerance to the mutations are assigned with green colors. The interference The sequence of the catalytic core is shown in the 5' to 3' direction. The contour graph represents the same data as in Fig 2.2-13.

Fig 2-2-13 and 2.2-14 show the interference values of all four libraries of 9F7 under all 5 conditions in bar graphs or a contour plot. The region in between A18 and T35 showed tolerance to all mutations in all the tested conditions. It was conceivable that the two regions (A1-G17 and C36-G40) are required to be connected via a linker. All 18 nucleotides were dispensable. Complete removal of the mutable region resulted in 2-fold decrease in the reaction $k_{\mathrm{obs}}$. When one, two or three nucleotides of the mutable region were retained the reaction rates improved and reached $80 \%$ of the reaction rate of the original 9F7. The reaction rates of the trimmed $9 \mathrm{~F} 7$ in the presence of $2 \mathrm{mM} \mathrm{Mn}^{2+}$ and $80 \mathrm{mM} \mathrm{Mg}^{2+}$ were $70 \%$ and $86 \%$ 
of the original enzyme. Importantly, $\mathrm{Tb}^{3+}$ accelerated the trimmed variant as effectively as the original 9F7 deoxyribozyme (Fig 2.2-15).

For 9F7 CoMA, the 2'-OH chemical tag had an inhibitory effect at several positions, when the ligation reaction was performed with $2 \mathrm{mM} \mathrm{Mn}^{2+}$ or $80 \mathrm{mM} \mathrm{Mg}^{2+}$. At low $\mathrm{Mn}^{2+}$, ribonucleotides were not well tolerated at A1, A2, G4, C8, G12, A14, C38 and G39. Addition of $10 \mu \mathrm{M} \mathrm{Tb}^{3+}$ to $2 \mathrm{mM} \mathrm{Mn}^{2+}$ relieved the $2^{\prime}-\mathrm{OH}$ interference at all positions. A similar result was obtained when $\mathrm{Tb}^{3+}$ was used in the presence of $\mathrm{Mg}^{2+}$ with two differences. Firstly, 2'-OH was tolerated at G39 even in the absence of $\mathrm{Tb}^{3+}$ and secondly, the detrimental effect of 2'-OH persisted at A1, A2 and A14. At $20 \mathrm{mM} \mathrm{Mn}^{2+}$, the 2'-OH group was tolerated at all positions.
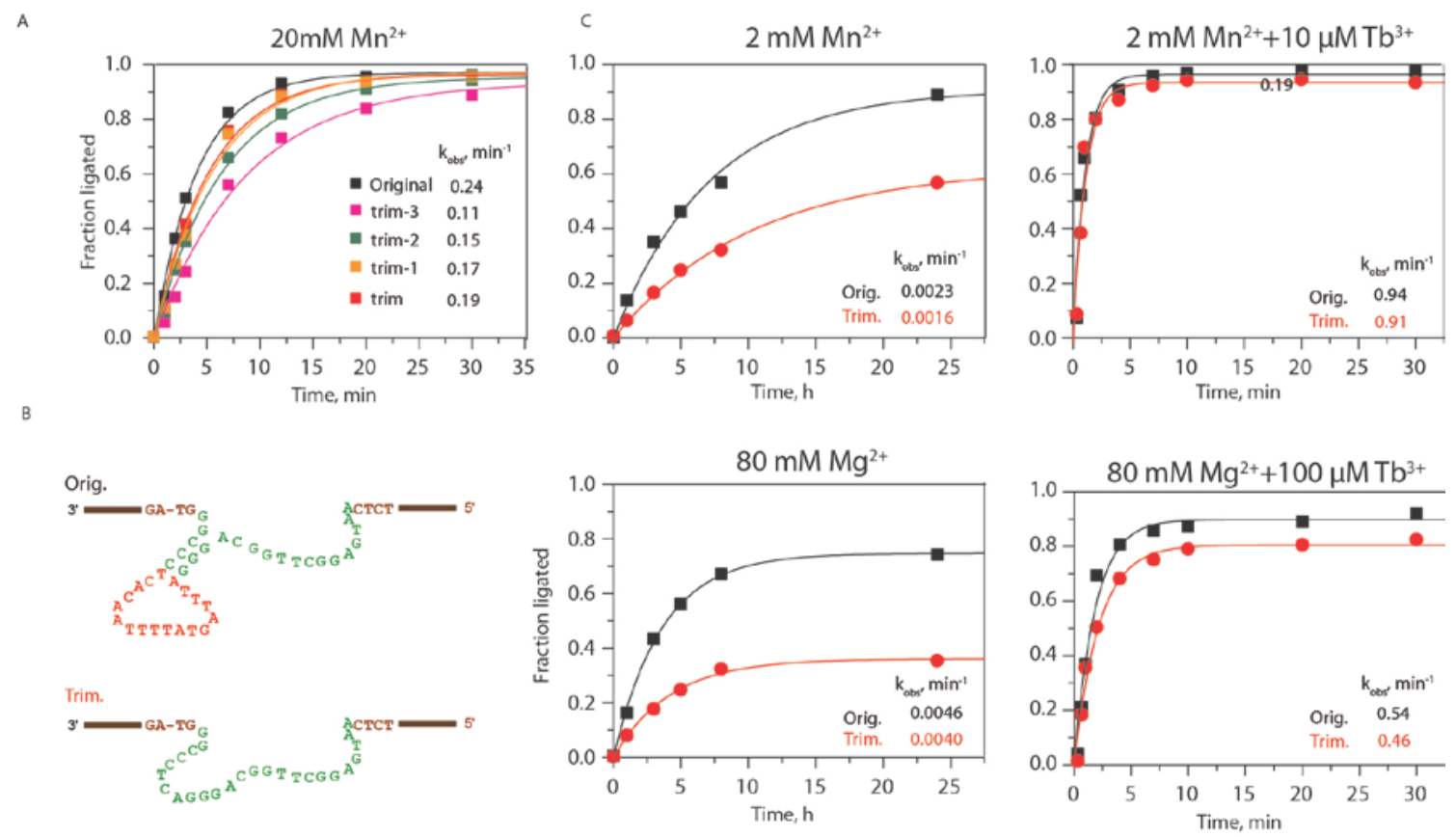

Fig 2.2-15 Kinetics of trimmed versions of $9 F 7$ A) kinetics in the presence of $20 \mathrm{mM} \mathrm{Mn}^{2+}$. All the tested trimmed versions of 9F7 were active. The sequence of each trimmed version is marked in Fig 2.2-14. B) The sequence of original and trimmed 9F7. The linker region (the region with toleration to all mutations) is marked with red color in the original sequence. Three nucleotides of the linker region (ACT) were remained in the chosen trimmed version of the enzyme for high activity. C) The kinetic activity of the original and trimmed $9 F 7$ in the presence of $2 \mathrm{mM} \mathrm{Mn}^{2+}, 80 \mathrm{mM} \mathrm{Mg}^{2+}$ in presence or absence of optimum $\mathrm{Tb}^{3+}$. 


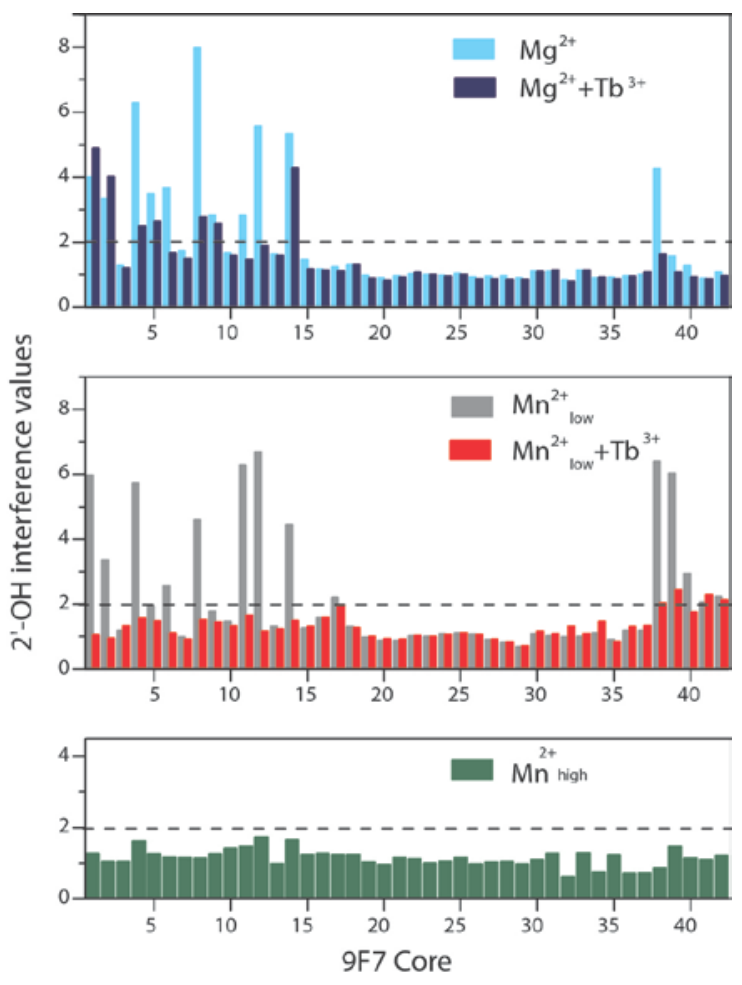

Fig 2.2-16 Interference values of the 2'-OH at individual positions of the catalytic core of 9F7 in the presence of $2 \mathrm{mM}$ $\mathrm{Mn}^{2+} \pm 10 \mu \mathrm{M} \mathrm{Tb} b^{3+}, 80 \mathrm{mM} \mathrm{Mg}^{2+} \pm 100 \mu \mathrm{M} \mathrm{Tb^{3+ }}$ and $20 \mathrm{mM} \mathrm{Mn}^{2+}$. Presence of high $\mathrm{Mn}^{2+}$ or optimum concentrations of $\mathrm{Tb}^{3+}$ relieved the detrimental effects of 2'-OH and reduced the interference values below 2.0 .

Several mutations were detrimental or reduced the activity of the enzyme when $\mathrm{Mn}^{2+}$ concentration was low (2 mM). Addition of $10 \mu \mathrm{M} \mathrm{Tb}^{3+}$ to the $2 \mathrm{mM} \mathrm{Mn}^{2+}$ or increase in the $\mathrm{Mn}^{2+}$ concentration (up to 20 $\mathrm{mM}$ ) relieved some of the mutation effects. Examples include: T9C, A5G, G15C, G16C, C38U, C13G, C13U, C36G, C37G, C38G and A14U. Presence of optimum $\mathrm{Tb}^{3+}$ concentration to the $\mathrm{Mg}^{2+}$ dependent reaction also relieved mutation effects at A2G, A5G, T9G, T10G, C36G, C37G, C38G T9C, A14C, G15C, G16C, G17C, A2U and C13U. In summary, high concentration of bivalent metal ion or presence of optimum $\mathrm{Tb}^{3+}$ increased the reaction rates and allowed slow mutants being present in the active fraction.

Several positions tolerated mutations only in $\mathrm{Mg}^{2+}$ or $\mathrm{Mn}^{2+}$ dependent reactions. Fig 2.2-17 is rearranged plot of Fig 2.2-14 to emphasize the similarities and differences of 9F7 nucleobase requirements for $\mathrm{Mg}^{2+}$ and $\mathrm{Mn}^{2+}$ dependent reactions. Individual mutations of any nucleotides in the region C13-G39 to $\mathrm{U}$ were better tolerated in the presence of $\mathrm{Mn}^{2+}$. Also, $\mathrm{Mn}^{2+}$ resulted in better tolerance of the region G15-C38 for C point mutations. C36-C38 mutations to G were better accommodated in the presence of $\mathrm{Mn}^{2+}$ as well. The only case that the interference value of the mutation for the $\mathrm{Mg}^{2+}$ dependent reaction was lower than $\mathrm{Mn}^{2+}$ dependent reaction was G4C. In general nucleobases requirement of the enzyme for the $\mathrm{Mg}^{2+}$ dependent reaction $\left(80 \mathrm{mM}\right.$ ) was higher than $\mathrm{Mn}^{2+}$ dependent reaction (when performed with $20 \mathrm{mM})$. 


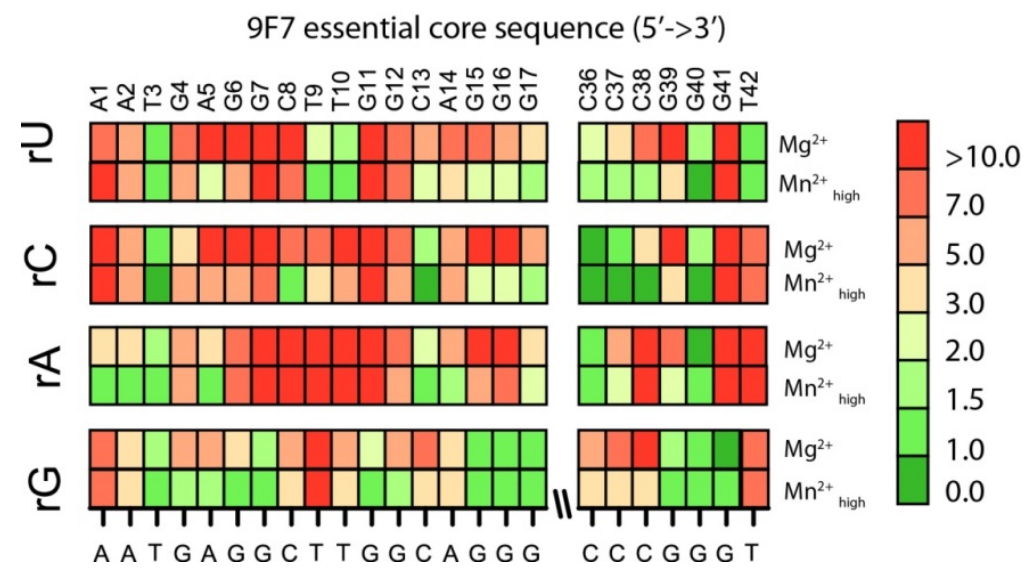

Fig 2.2-17 Interference values for of the necessary nucleobases of $9 F 7$ for $\mathrm{Mg}^{2+}(80 \mathrm{mM})$ and $\mathrm{Mn}^{2+}(20 \mathrm{mM})$ dependent catalysis. The site of the deleted linker is marked by two dashes. The plot is an excerpt of the plot shown in 2.2-14 which is rearranged for better comparison between $\mathrm{Mg}^{2+}$ and $\mathrm{Mn}^{2+}$ dependent reactions.

In summary, CoMA revealed nucleotide positions which are necessary for catalysis in all conditions. Position G4 was an example of those nucleotides. In addition, CoMA provided insights about nucleobases at which the mutations are only tolerated under certain conditions e.g. in presence of $\mathrm{Tb}^{3+}$ or high concentration of $\mathrm{Mn}^{2+}$. To further explore the interactions of 9F7 catalytic core with metal ions we used the spectroscopic properties of $\mathrm{Tb}^{3+}$. Inner-sphere coordination of $\mathrm{Tb}^{3+}$ to the trimmed version of the 9F7 catalytic core was quantitatively analyzed using sensitized luminescence of $\mathrm{Tb}^{3+}$.

\subsubsection{Sensitized luminescence of $\mathrm{Tb}^{3+}$}

We explored sensitized luminescence of $\mathrm{Tb}^{3+}$ to probe $\mathrm{Tb}^{3+}$ interactions with trimmed 9F7 in complex with its RNA substrates. We chose a pre-ligation complex formed with standard active donor substrate and inactivated acceptor substrate, containing 2'-deoxyadenosine at the branch-site (R013). Addition of $\mathrm{Tb}^{3+}$ to annealed complexes under various divalent metal ion conditions resulted in an increase in $\mathrm{Tb}^{3+}$ luminescence upon excitation at $284 \mathrm{~nm}$. Quantitative information on $\mathrm{Tb}^{3+}$-binding was obtained by titration experiments up to $200 \mu \mathrm{M} \mathrm{Tb}^{3+}$, in the presence of $1-100 \mathrm{mM} \mathrm{Mg}^{2+}$ or $1-10 \mathrm{mM} \mathrm{Mn}^{2+}$. The intensity changes of $\mathrm{Tb}^{3+}$ luminescence signal at $543 \mathrm{~nm}$ was measured over time.

It is noteworthy that different binding kinetics of $\mathrm{Tb}^{3+}$ was observed depending on the type of the divalent metal ions and concentration of $\mathrm{Tb}^{3+}$. In the presence of $\mathrm{Mn}^{2+}$, a fast increase in emission was followed by a fast and further slow decrease of characteristic signature within 10 min (Fig 2.2-18 B). The emission intensities after an equilibration time of $10 \mathrm{~min}$ for each data point are plotted against $\mathrm{Tb}^{3+}$ concentration in Fig 2.2-18 C. 
A
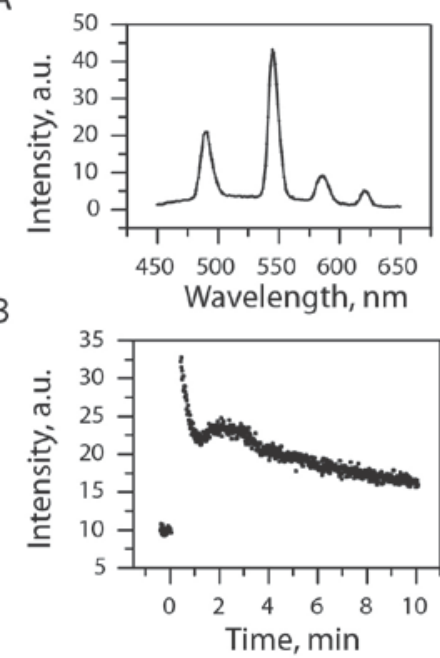

C

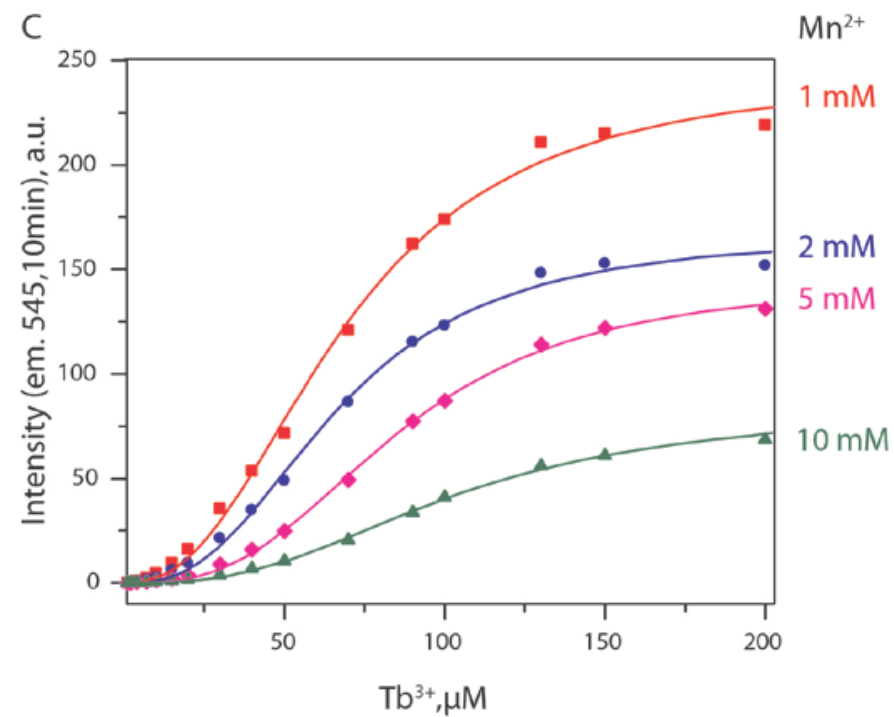

Fig 2.2-18 Sensitized $\mathrm{Tb}^{3+}$ luminescence in competition with $\mathrm{Mn}^{2+}$. A) A typical emission spectrum of sensitized $\mathrm{Tb}^{3+}$ in the presence of $2 \mu M$ DNA. The excitation wavelength was set at $284 \mathrm{~nm}$. B) Kinetics of luminescence intensity at $545 \mathrm{~nm}$ upon excitation at 284. Upon addition of each $\mathrm{Tb}^{3+}$ aliquots the initial increase in the luminescence was rapidly decreased within the first $2 \mathrm{~min}$. The decrease in the luminescence was further continued with slower rate. C) $\mathbf{T b}^{3+}$ titration in the presence of $\mathrm{Mn}^{2+}$. For consistency the luminescence intensities are reported after 10 min equilibration time for each $\mathbf{T b}^{3+}$ addition events.

In the presence of $\mathrm{Mg}^{2+}$, three different binding modes were observed. At low concentrations of $\mathrm{Tb}^{3+}$ $(<20 \mu \mathrm{M})$, an initial increase was followed by an exponential decay to almost the initial value within 1-3 min. At slightly elevated $\mathrm{Tb}^{3+}$ concentrations (between 20 and $60 \mu \mathrm{M}$ ), the signal intensity increased rapidly and stayed at the elevated level for at least $10 \mathrm{~min}$. Higher $\mathrm{Tb}^{3+}$ concentrations $(>60 \mu \mathrm{M})$, resulted in a slower response of the emission intensity.

As anticipated for both $\mathrm{Mg}^{2+}$ and $\mathrm{Mn}^{2+}$, competitive $\mathrm{M}^{2+}$ and $\mathrm{Tb}^{3+}$ binding, the maximal emission intensities and the apparent affinity for $\mathrm{Tb}^{3+}$ i.e. $\left[\mathrm{Tb}^{3+}\right]_{1 / 2}$, decreased with rising divalent metal ion concentrations. Table 2.2-3 summarizes the fit values for $\mathrm{Tb}^{3+}$ titration in the presence of increasing concentrations of bivalent metal ions.

Table 2.2-3 Fit values of the titration curves from Fig 2.2-18 and 2.2-19. The curves were fit to the equation $I=I_{\max } \bullet$ $\left[\mathbf{T b}^{3+}\right]^{\mathrm{n}} /\left(\left[\mathbf{T b}^{3+}\right]_{1 / 2}{ }^{\mathrm{n}}+\left[\mathbf{T b}^{3+}\right]^{\mathrm{n}}\right)$.

\begin{tabular}{lllll}
\multicolumn{2}{l}{ divalent metal ion } & Imax & {$\left[\mathrm{Tb}^{3+}\right]_{1 / 2}(\mu \mathrm{M})$} & $\mathrm{n}$ \\
\hline $\mathrm{Mn}^{2+}$ & $1 \mathrm{mM}$ & 245 & 69 & 2.4 \\
& $2 \mathrm{mM}$ & 167 & 67 & 2.6 \\
& $5 \mathrm{mM}$ & 145 & 86 & 2.8 \\
& $10 \mathrm{mM}$ & 82 & 101 & 2.7 \\
\hline $\mathrm{Mg}^{2+}$ & $1 \mathrm{mM}$ & 508 & 93 & 2.9 \\
& $10 \mathrm{mM}$ & 493 & 100 & 2.8 \\
& $20 \mathrm{mM}$ & 436 & 114 & 2.6 \\
& $100 \mathrm{mM}$ & 373 & 139 & 2.6
\end{tabular}



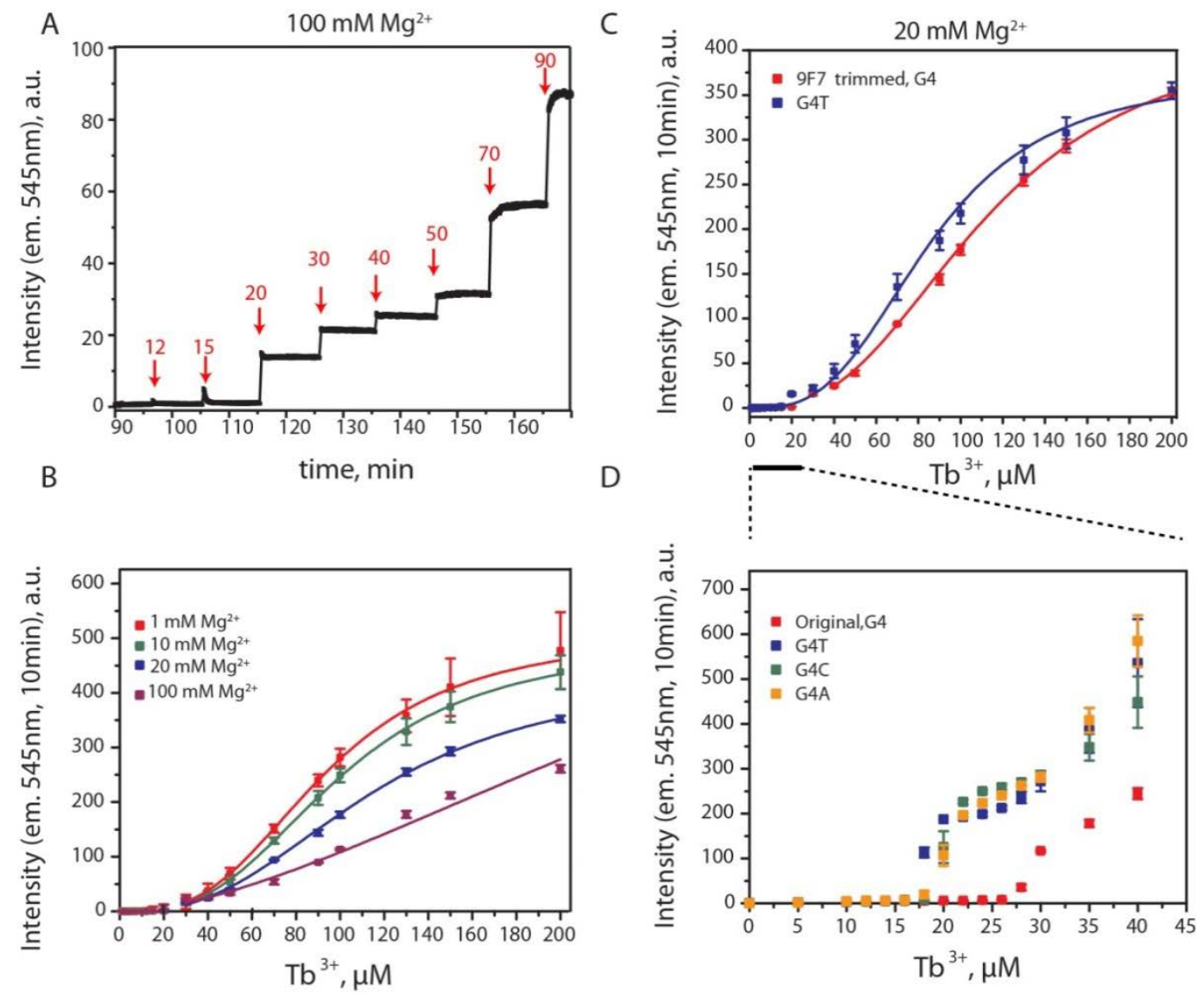

Fig 2.2-19 Sensitized luminescence of $\mathrm{Tb}^{3+}$ in competition with $\mathrm{Mg}^{2+}$. A) Typical kinetic of $\mathrm{Tb}^{3+}$ luminescence at various $\left[\mathrm{Tb}^{3+}\right]$ when the enzyme is folded in the presence of $\mathrm{Mg}^{2+}$. The final concentration of $\mathrm{Tb}^{3+}$ after each addition is indicated in $\mu \mathrm{M}$. B) $\mathrm{Tb}^{3+}$ titration in the presence of 1-10 $\mathrm{mM} \mathrm{Mg}^{2+}$. C) $\mathrm{Tb}^{3+}$ titration in the presence of $20 \mathrm{mM} \mathrm{Mg}^{2+}$ to the active trimmed 9F7 and an inactive mutant G4T. The detector voltage was set at $600 \mathrm{~V}$. D) $\mathrm{Tb}^{3+}$ titration to the active trimmed 9F7 and inactive G4 mutants in the presence of $20 \mathrm{mM} \mathrm{Mg}^{2+}$. The detector voltage was set at $800 \mathrm{~V}$.

Table 2.2-4 Kinetics of trimmed 9F7 in comparison to G4 mutants.

\begin{tabular}{llllll}
\multicolumn{5}{l}{ Position 4 of the 9F7 core } \\
\hline Conditions & $\mathrm{G}$ & $\mathrm{A}$ & $\mathrm{C}$ & $\mathrm{U}$ & \\
\hline $\mathrm{Mn}^{2+}{ }_{\text {high }}$ & 0.88 & 0.12 & 0 & 0 & \% in 1h \\
& $\mathbf{0 . 9 5}$ & $\mathbf{0 . 6 6}$ & $\mathbf{0}$ & $\mathbf{0}$ & \%in 24h \\
$\mathrm{Mn}^{2+}{ }_{\text {low }}+\mathrm{Tb}^{3+}$ & 0.91 & 0.23 & 0 & 0 & \\
& $\mathbf{0 . 9 5}$ & $\mathbf{0 . 7 5}$ & $\mathbf{0}$ & $\mathbf{0}$ & \\
$\mathrm{Mg}^{2+}$ & 0.23 & 0 & 0 & 0 & \\
$\mathrm{Mg}^{2+}+\mathrm{Tb}^{3+}$ & 0.88 & 0 & 0 & 0 & \\
& $\mathbf{0 . 9 1}$ & $\mathbf{0}$ & $\mathbf{0}$ & $\mathbf{0}$ &
\end{tabular}

Based on CoMA, G4 was potential candidate nucleotide for coordination of metal ions; the sensitivity to the 2'-OH ribose modification was relieved with $\mathrm{Tb}^{3+}$, no mutations were allowed with $\mathrm{Mg}^{2+}$, and a purine nucleotide must be retained for activity with $\mathrm{Mn}^{2+}$. Kinetic experiments on point mutants of G4 
proved CoMA data (Table 2.2-4). We further analyzed G4 mutants according to their $\mathrm{Tb}^{3+}$-binding properties. Luminescence response upon $\mathrm{Tb}^{3+}$ titration (up to $200 \mu \mathrm{M}$ ) for inactive G4T mutant had a slight left-shift titration curve in comparison to the trimmed $9 F 7$ (G4) in the presence of $20 \mathrm{mM} \mathrm{Mg}^{2+}$. Significant differences were observed at low $\mathrm{Tb}^{3+}$ concentrations. Active trimmed 9F7 (G4) showed persistence luminescence increase only above $25 \mu \mathrm{M} \mathrm{Tb}^{3+}$ but all three G4 inactive mutants showed this transition at $\mathrm{Tb}^{3+}$ concentration below $20 \mu \mathrm{M}$ (Fig 2.2-19).

To summarize, sensitized luminescence of $\mathrm{Tb}^{3+}$ confirmed presence of specific binding sites for $\mathrm{Tb}^{3+}$ in the catalytic core of the enzyme. Additionally, it was shown that bivalent metal ions and $\mathrm{Tb}^{3+}$ compete for the peripheral metal ion binding sites. Finally, the CoMA data for involvement of position G4 in formation of the catalytically important bivalent metal ion binding site was supported with the luminescence data.

\subsubsection{F7 folding studies by DMS and DNase probing assays}

To further investigate the deoxyribozyme folding and interactions with metal ions we performed footprinting assays in presence or absence of metal ions. DNase I probing is based on the accessibility of DNA backbone for cleavage by the protein enzyme DNase I. DMS probing is one of the four reactions of Maxam- Gilbert sequencing ${ }^{141}$. Dimethylsulfate (DMS) alkylates the $\mathrm{N}^{7}$ of guanines, $\mathrm{N}^{1}$ of adenines and $\mathrm{N}^{3}$ of cytosines. Additionally, DMS is reactive with $\mathrm{N}^{3}$ and $\mathrm{N}^{1}$ of the enol-tautomers of uracils and guanines ${ }^{142}$. The alkylated positions are identified by primer extension ${ }^{142}$ or site specific cleavage by aniline or piperidine treatment. The latter is specific for the methylations at $\mathrm{N}^{7}$ of guanines and is used in this study. Methylation at $\mathrm{N}^{7}$ of guanosines enhances formation of apurinic sites by weakening the glycosidic bond. Upon piperidine treatment, $\beta$-elimination of the ribose is catalysed and the open ring of the apurinic ribose is released ${ }^{143}$. The cleavage products are 3' phosphorylated DNA strand 5' of the displaced guanosine and 5' phosphorylated DNA strand 3' of the guanosine (Fig 2.2$20)^{144}$.

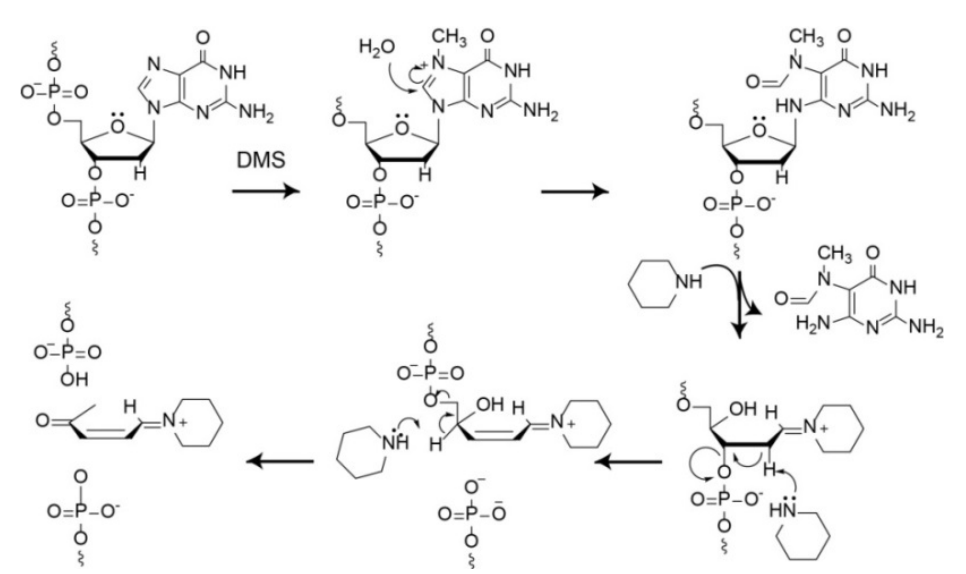

Fig 2.2-20 DMS-mediated methylation of $\mathrm{N}^{7}$ of guanines in a DNA strand and piperidine cleavage at the site of methylation. 
Three different sample compositions were used to compare different folding states of the DNA. First, the DNA enzyme was analyzed as the only nucleic acid component in the sample (9F7min alone, $D^{t}$ ), second in complex with inactivated substrates (standard acceptor RNA and 5'-monophsphorylated donor RNA, iS), and third in complex with the 2',5'-branched RNA product (brP). The accessibility of nucleotides in the catalytic region of trimmed 9F7 was probed in buffer without divalent metal ions and in the presence of $20 \mathrm{mM} \mathrm{Mn}^{2+}$ (Fig 2.2-21).

DMS mediated methylation at $\mathrm{N}^{7}$ of guanosines of 9F7 was reduced G4, G6, G7, G11, G12 and G15 in the folded brP sample in the presence of $\mathrm{Mn}^{2+}$. The protection was less pronounced in iS complex. G16 and G17 remained accessible in all samples, suggesting that these nucleotides are not actively participating in formation of the active structure.

T20 in the trimmed catalytic core of 9F7 corresponds to T35 in the original length 9F7. Superscript " $t$ " stands for trimmed when the number of the nucleotide position is changed after trimming. DNase I resulted in a strong cleavage band at $\mathrm{T} 20^{\mathrm{t}}$. High accessibility of this position was consistent with the CoMA data that suggested that this nucleotide is not involved in essential interactions or catalysis. Digestion at G7 was blocked in both post-hybridization states i.e. iS and brP, even in the absence of $\mathrm{Mn}^{2+}$, while cleavage at position T9 was unaffected. The DNase-mediated strand break at G17 was enhanced in the brP complex, while reduced intensity was observed at C21. Strikingly, cleavage at most of the positions was strongly diminished in the presence of $\mathrm{Mn}^{2+}$, especially for brP complex although $\mathrm{Mn}^{2+}$ is known to increase the activity of DNase $\mathrm{I}^{145}$.

Altogether, DMS and DNase probing assays provided further supports to the CoMA data. Structural changes upon presence of buffer and metal ions were reflected on the DNase I digestion or piperidine cleavage patterns. Investigations of the deoxyribozyme 9F7 was further continued by phosphorothioate rescue experiments. 


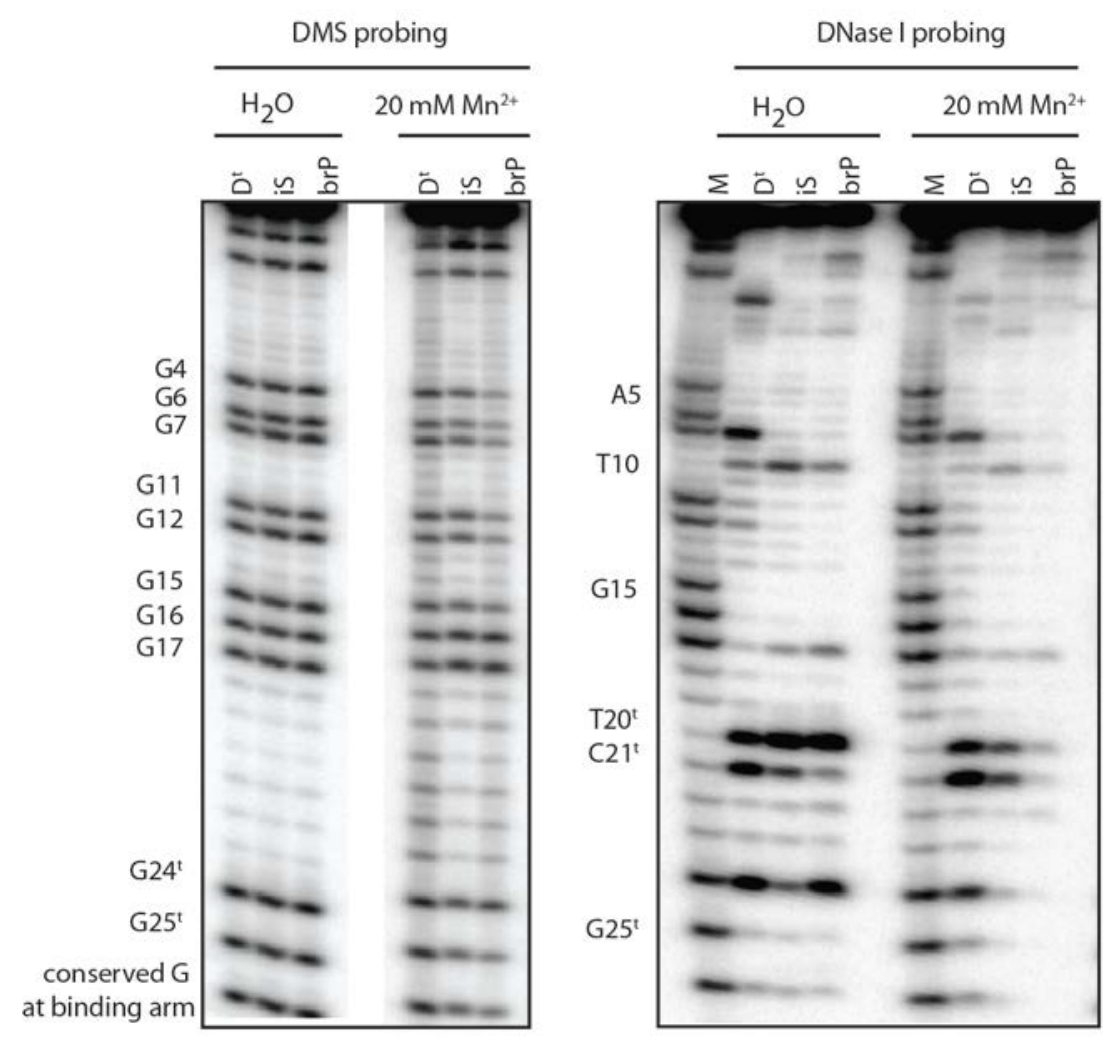

Fig 2.2-21 DMS and DNase I probing assays of trimmed 9F7 in presence and absence of buffer and metal ions. Three samples have been subjected for probing assays. 1) $D^{t}$ is the trimmed 9F7 DNA as the only nucleic acid component of the sample. 2) is is the complex of $D^{t}$ with inactivated substrates. 3 ) brP is the complex of the $D^{t}$ with branched product of 9F7. Numberings are based on trimmed 9F7. The numbers which are different before and after trimming are marked with superscript letter " $t$ ".

\subsubsection{Phosphorothioate rescue experiments}

To further explore the specific synergistic effects of bivalent metal ions and trivalent rare earth elements on activation of the RNA substrates, we investigated the catalytic activity of the 9F7 using phosphorothioate-modified donor substrates. One oxygen atom at either the $\alpha$ or $\gamma$ phosphate was replaced by sulfur in the donor RNA. Phosphorothioate (PS) interference and metal ion rescue experiments have the potential to reveal crucial information about metal ion interactions in the active site. ${ }^{146}$ Installation of $\gamma$-PS can be easily achieved by in vitro transcription using $\gamma$-S-GTP. In contrast, transcription with $\alpha$-S-GTP results in incorporation of PS at the 5'-terminal $\alpha$-phosphate and at all other guanosines in the strand. To avoid cumulative effects of more than one PS in the substrate, we designed an alternative donor substrate ( $\operatorname{Tr} 14)$ that contained only one guanosine at the 5'-end, and otherwise consisted of only A, C and U.

Replacement of the $\gamma$-phosphate by PS in donor RNA (Tr12) caused a considerably reduced ligation rate compared to standard tirphosphorylated $\operatorname{Tr} 12\left(k_{\mathrm{obs}} 3 \times 10^{-5}\right.$ vs $0.003 \mathrm{~min}^{-1}$ in presence of $80 \mathrm{mM}$ $\mathrm{Mg}^{2+} ; k_{\text {obs }} 0.02$ vs $0.30 \mathrm{~min}^{-1}$ in presence of $20 \mathrm{mM} \mathrm{Mn}^{2+}$ ). Addition of $\mathrm{Tb}^{3+}$ did not change the outcome of the reaction. Addition of $1 \mathrm{mM} \mathrm{Cd}^{2+}$ to the reaction with $80 \mathrm{mM} \mathrm{Mg}^{2+}$ did not significantly improve the ligation rate. In the presence of $\mathrm{Cd}^{2+}$ and $\mathrm{Mg}^{2+}$ the reaction rates were $2 \times 10^{-4} \mathrm{~min}^{-1}$ for donor 
substrate bearing $\gamma$-phosphate (PS) and $0.005 \mathrm{~min}^{-1}$ for triphosphorylated substrate (PO). Addition of 2 $\mathrm{mM} \mathrm{Mn}^{2+}$ to the reaction with $80 \mathrm{mM} \mathrm{Mg}^{2+}$ accelerated ligations of both PO and PS RNAs by a factor of ca 10-20, which indicated no specific rescue effect for the $\gamma$ PS $\left(k_{\mathrm{obs}} 0.028\right.$ for PO and $4 \times 10^{-4}$ for PS in the presence of $80 \mathrm{mM} \mathrm{Mg}^{2+}$ and $2 \mathrm{mM} \mathrm{Mn}^{2+}$ ). In the presence of higher $\mathrm{Mn}^{2+}$ concentration (20 mM $\mathrm{Mn}^{2+}$ with $80 \mathrm{mM} \mathrm{Mg}^{2+}$ ), the ligation of the PS substrate was accelerated 250-fold, while the reaction of the PO substrate was only ca 70-fold faster than with $\mathrm{Mg}^{2+}$ alone (Fig 2.2-22).

The alternative donor substrate with one guanine in the sequence, " $1 G$ ", was ligated less efficiently than standard donor substrate under the standard reaction conditions ( $k_{\mathrm{obs}} 0.03 \mathrm{vs} 0.3 \mathrm{~min}^{-1}$ in the presence of $20 \mathrm{mM} \mathrm{Mn}{ }^{2+}$ ). Replacement of the $\alpha$ phosphate with PS resulted in comparable ligation rates using $\mathrm{Mn}^{2+}$ as cofactor $\left(k_{\mathrm{obs}} 0.05 \mathrm{~min}^{-1}\right)$, while PS at the $\gamma$ position again severely inhibited the reaction $\left(k_{\mathrm{obs}}\right.$ $0.0004 \mathrm{~min}^{-1}$ ). Upon addition of $1 \mathrm{mM} \mathrm{Cd}^{2+}$, the $k_{\mathrm{obs}}$ for the $\alpha$-PS analog of " $1 \mathrm{G}$ " substrate increased ca 3-fold ( $\left.k_{\mathrm{obs}} 0.13 \mathrm{~min}^{-1}\right)$, while the ligation rate of the unmodified substrate remained unaffected $\left(k_{\mathrm{obs}} 0.03\right.$ $\mathrm{min}^{-1}$ ). Presence of $10 \mu \mathrm{M} \mathrm{Tb}{ }^{3+}$ had a comparable 3-fold effect for the $\alpha$-PS substrate, while ligation of the unmodified $\operatorname{Tr} 14$ was ca 10 -fold faster than in the absence of $\mathrm{Tb}^{3+}$. Collectively, these results point toward important interactions at the $\alpha$ - and $\gamma$-phosphates of the donor RNA, although the PS and rescue effects were less pronounced than for other metalloribozymes ${ }^{147}$. 

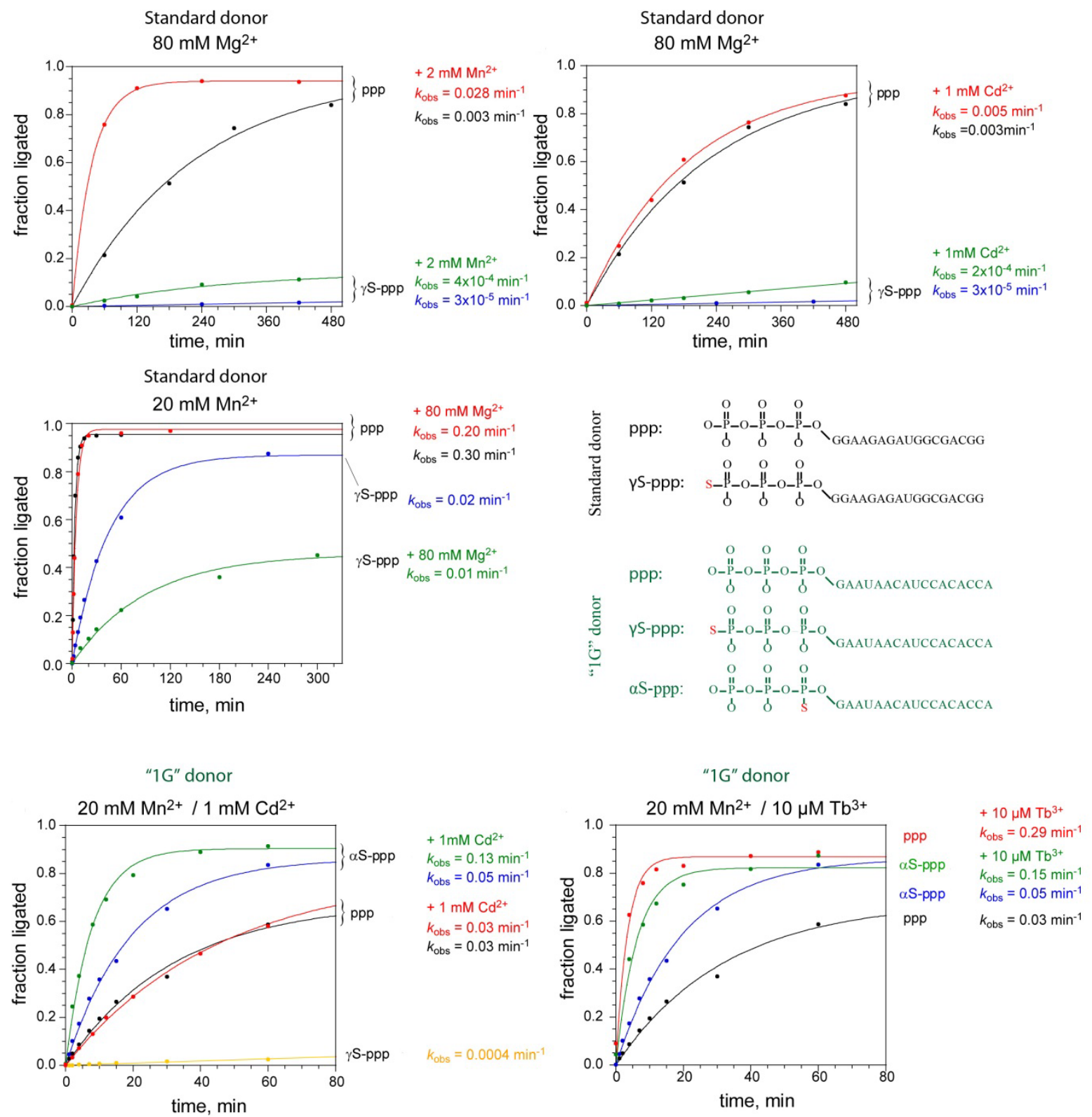

Fig 2.2-22 Phosphorothioate effects and metal ion rescue attempts for 9F7-catalyzed ligation of standard acceptor RNA with standard or alternative " $1 G$ " donor RNA containing $\gamma$ - and $\alpha$-phosphorothioate.

\subsection{7 $\quad \mathrm{Tb}^{3+}$ mediated acceleration of other enzymes}

In addition to 9F7, 9F13 (from the same deoxyribozyme family), 6CE8 ${ }^{27}$ (the deoxyribozyme capable of branch formation with any nucleotide at branch site) and three-helix Junction (3HJ) forming deoxyribozymes, $7 \mathrm{~S} 11^{23}$ and $10 \mathrm{DM} 24^{28}$, have been investigated for their response to $\mathrm{Tb}^{3+}$. In presence of $\mathrm{Mg}^{2+}$ 7S11 and 10DM24 were accelerated upon presence of $100 \mu \mathrm{M} \mathrm{Tb}{ }^{3+}$ (Fig 2.2-23 A shows the data for 7S11). Additionally, $\mathrm{Mn}^{2+}$ dependent reaction of 7S11 and 9F13 were best accelerated in the presence of 5-20 $\mu \mathrm{M} \mathrm{Tb}^{3+}$ (Fig 2.2-23 B and 2.2-24). Addition of $150 \mu \mathrm{M} \mathrm{Tb}{ }^{3+}$ to $\mathrm{Mn}^{2+}$ dependent reaction of 6CE8 inhibited the reaction. Further investigation of the enzyme 6CE8 is required to confirm if $\mathrm{Tb}^{3+}$ only inhibits the enzyme. High concentrations of $\mathrm{Tb}^{3+}(\sim 150 \mu \mathrm{M})$ were inhibitory for $\mathrm{Mn}^{2+}$ dependent reaction of the other three enzymes i.e. 9F7, 9F13, and 7S11. It can be predicted that in 
the case of 6CE8 not only the enzyme is not inhibited at low concentrations of $\mathrm{Tb}^{3+}$ (ca. $10 \mu \mathrm{M}$ ) but also is accelerated. $\mathrm{Tb}^{3+}$ acceleration of 10DM24 enzymatic reaction was further studied and employed for single GTP attachment to RNA molecules (Section 3.1).

A

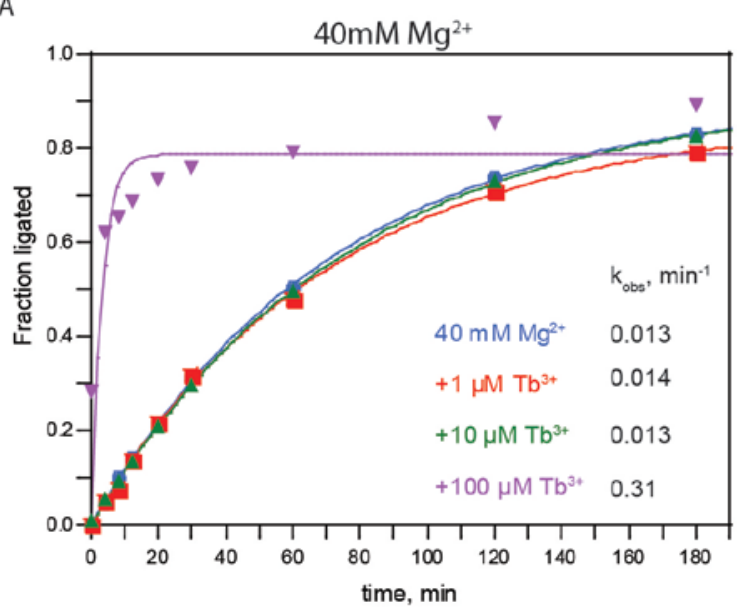

B

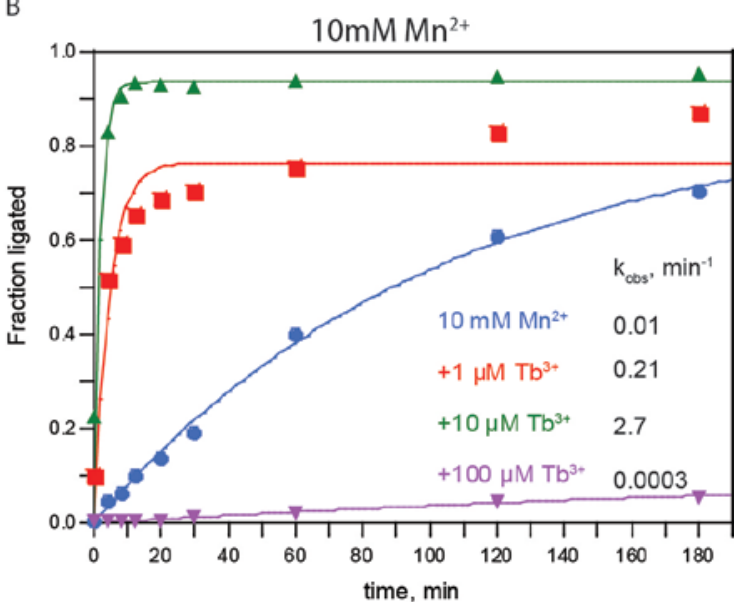

Fig 2.2-23 $\mathrm{Tb}^{3+}$ mediated acceleration of $\mathrm{Mg}^{2+}$ and $\mathrm{Mn}^{2+}$ dependent reactions of $7 \mathrm{~S} 11 . \mathrm{Mg}^{2+}$ dependent reaction was accelerated upon presence of $100 \mu \mathrm{M} \mathrm{Tb}^{3+}$. $\mathrm{Mn}^{2+}$ dependent reaction was accelerated upon presence of 1-10 $\mu \mathrm{M} \mathrm{Tb}^{3+}$.

Further investigations have been performed on 9F13 deoxyribozyme employing combinatorial mutation interference analysis in the presence of $20 \mathrm{mM} \mathrm{Mn}^{2+}$ (Fig 2.2-25). 9F13 CoMA revealed complete mutation tolerance for G11-T15 and G20-T33. A couple of deletion mutants (appendix I) have been tested for trimming 9F13. When the region G20-T33 was completely removed the enzymatic activity of the enzyme reduced significantly ( $10 \%$ product in 8 h). In contrast, complete removal of T13 and T15 had slight acceleration effect ( $k_{\text {obs }} 0.41$ vs $0.24 \mathrm{~min}^{-1}$ trimmed and original $9 \mathrm{~F} 13$ at $25^{\circ} \mathrm{C}$ ). Removal of T13-T15 and replacement of the region G20-T33 with two thymidines (9F13 trim) improved the reaction rate by 4 -fold ( $k_{\mathrm{obs}} 0.99$ vs $0.24 \mathrm{~min}^{-1}$ for trimmed and original $9 \mathrm{~F} 13$ at $25^{\circ} \mathrm{C}$ and in the presence of $20 \mathrm{mM} \mathrm{Mn}^{2+}$ ). The trimmed 9F13 had only 23 nucleotides and contained 12 guanines in the catalytic core (Fig 2.2-26).

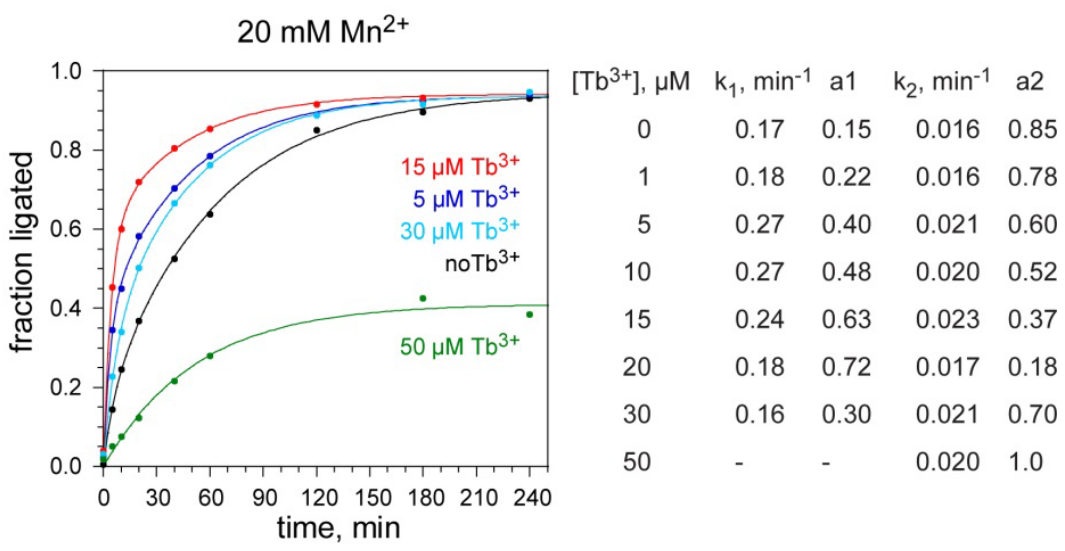

Fig 2.2-24 Kinetics of original 9F13 deoxyribozyme in the presence of $20 \mathrm{mM} \mathrm{Mn^{2+ }}$ and micromolar concentrations of

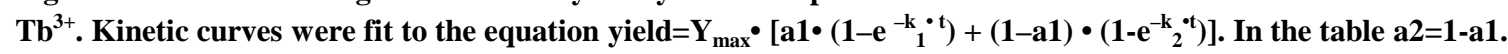



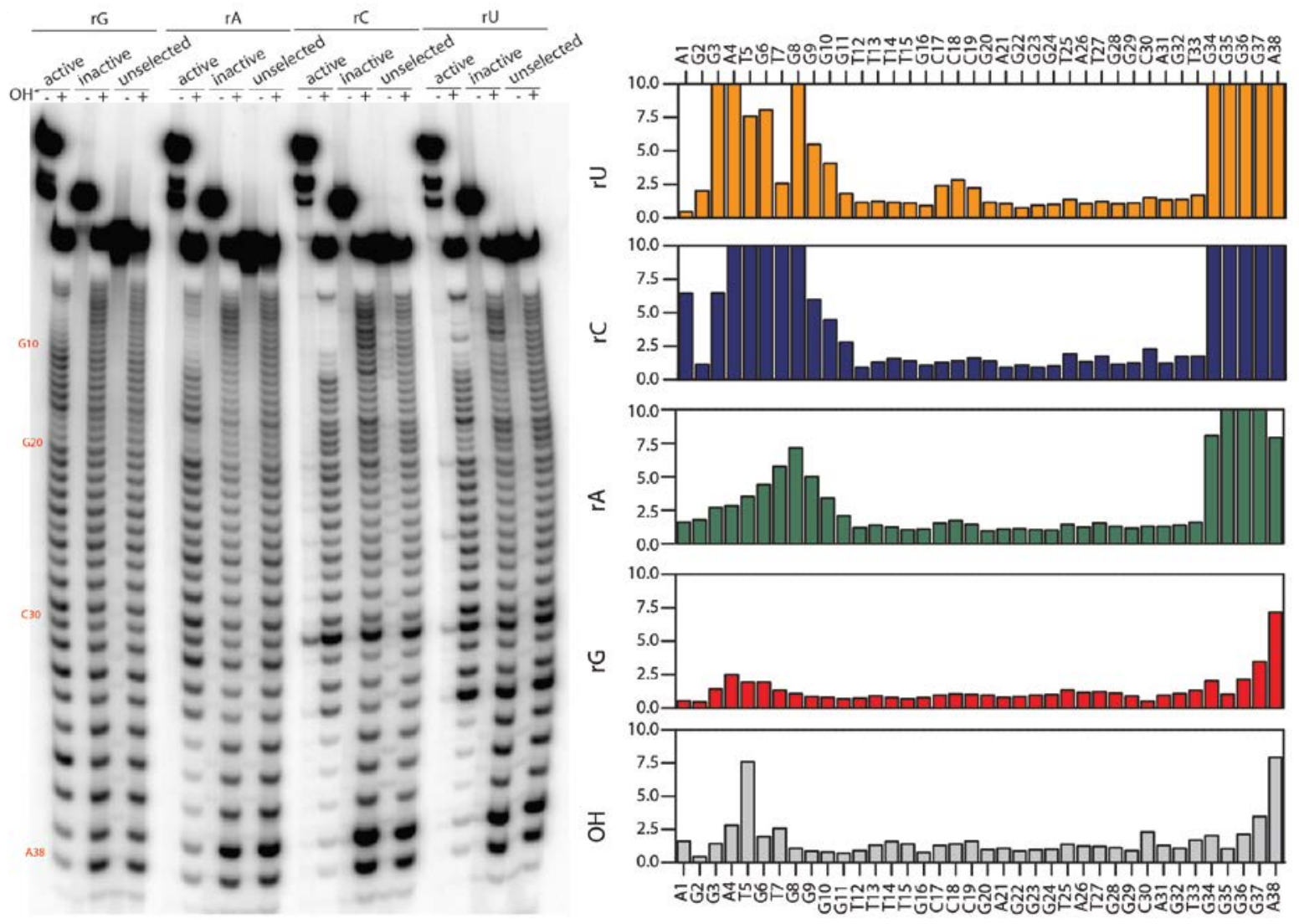

Fig 2.2-25 Combinatorial mutation interference analysis of $9 \mathrm{F13}$ in the presence of $20 \mathrm{mM} \mathrm{Mn}^{2+}$. $\mathrm{Left}^{\text {is }}$ the autoradiogram of the hydrolysis pattern of active, inactive and unselected mutant libraries of 9F13. Right is the interference values of each mutant library for individual positions of 9F13 catalytic core.

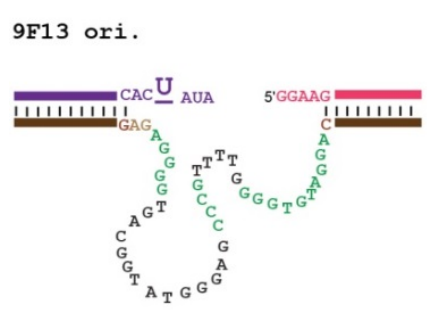

$9 \mathrm{~F} 13$ trim.
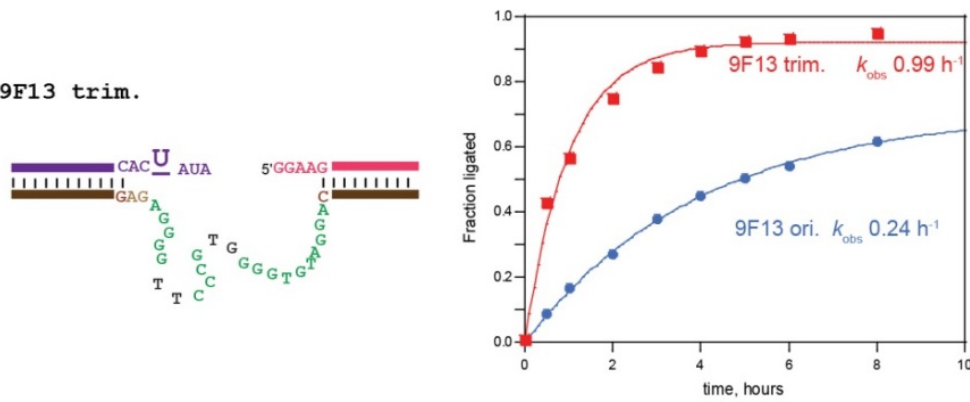

Fig 2.2-26 9F13 trimming kinetics. The original sequence and trimmed sequence of 9F13 are shown in the left. The uridine branch site is marked bold and underlined. Kinetic experiments have been performed in the presence of $20 \mathrm{mM}$ $\mathrm{Mn}^{2+}$ at $25^{\circ} \mathrm{C}$. The kinetic data shows 4 -fold rate acceleration upon removal of the mutable region.

In summary, our investigations demonstrated that the $\mathrm{Tb}^{3+}$ dependent acceleration of 2',5'- branched RNA formation is not a specific case for 9F7 but also is valid for another member of 9F7 family (9F13) and also for three-helix junction forming deoxyribozymes i.e. 7S11 and 10DM24.

\subsubsection{Discussion}

$\mathrm{Tb}^{3+}$ was shown to be an effective accelerator for catalysis of 2',5' phosphodiester bond formation with 9F7 and 9F13, 7S11 and 10DM24. $\mathrm{Tb}^{3+}$ mediated acceleration was investigated in details for 9F7. The kinetics of the enzyme was enhanced up to 10000-fold at low concentrations of bivalent metal ions. The 
affinity of the enzyme to the bivalent metal ions was increased upon presence of optimum concentrations of $\mathrm{Tb}^{3+}$. Bivalent metal ions were shown to be necessary for 9F7 catalysis suggesting their involvement in the catalytic center of the enzyme (Fig 2.2-27).
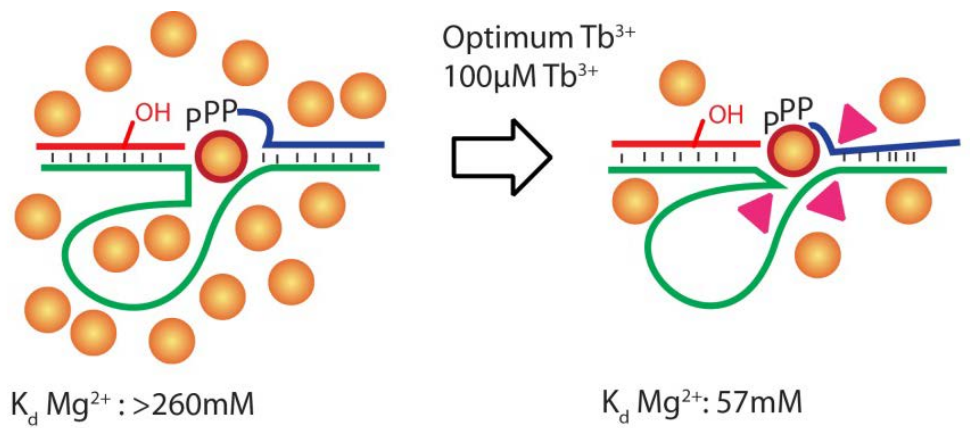

Fig 2.2-27 The effect of $\mathrm{Tb}^{3+}$ on $9 F 7$ affinity to the active $\mathrm{M}^{2+}$.Optimum $\mathrm{Tb}^{3+}$ increases the affinity of the enzyme to its active metal ion. $\mathrm{Mg}^{2+}$ and $\mathrm{Tb}^{3+}$ ions are shown in orange and pink. The $\mathrm{Mg}^{2+}$ ion involved in the formation of the catalytic center is marked with red border. Presence of optimum $\mathrm{Tb}^{3+}$ facilitates formation of the catalytic center at lower concentrations of $\mathrm{Mg}^{2+}$. At the same time, the increased affinity of the catalytically important metal ion binding site to the bivalent metal ion accelerates the reaction.

The significance of the $\mathrm{Tb}^{3+}$-mediated acceleration of RNA ligation reaction is clearer when compared to previous reports of the $\mathrm{Tb}^{3+}$ and other lanthanides effect on ribozymes and deoxyribozymes. The RNA cleaving deoxyribozyme 8-17 which was reported to be active in the presence of $\mathrm{Mg}^{2+}, \mathrm{Zn}^{2+}, \mathrm{Pb}^{2+}$ or $\mathrm{Co}^{2+}$ was inhibited upon addition of micromolar $\mathrm{Tb}^{3+}$ concentrations ${ }^{135}$. Magnesium dependent reaction of hammerhead ribozyme was reversibly inhibited upon presence of $\mathrm{Tb}^{3+}$ in micromolar concentrations $^{148}$. For the nucleolytic ribozymes, under special conditions small acceleration effects were reported. For example, when tested in the presence of high concentrations of $\mathrm{Na}^{+}$or spermidine (> $1 \mathrm{M})$, low amounts of $\mathrm{Tb}^{3+}(<25 \mu \mathrm{M})$ accelerated the hairpin ribozyme-catalyzed RNA cleavage up to 2.5-fold, while higher $\mathrm{Tb}^{3+}(>30 \mu \mathrm{M})$ inhibited the ribozyme ${ }^{126}$. Upto 30-fold increase in the leadzyme activity was reported by Ohmichi et $\mathrm{al}^{133}$ with an optimal combination of $\mathrm{Nd}^{3+}: \mathrm{Pb}^{2+}(1: 3)$.

The 9F7 and 9F13 lanthanide mediated acceleration was observed in a narrow range of lanthanide concentrations. Interestingly, the optimum range was dependent on the identity of the active bivalent metal ion, disrespect to $\mathrm{M}^{2+}$ concentration. Although the ratio of $\left[\mathrm{Tb}^{3+}\right]$ : $\left[\mathrm{M}^{2+}\right]$ was shown not to be important, the ratio of [Metal ions]: [nucleic acid components] was crucial for fast ligation. As a rule of thumb, our data suggests $\left[\mathrm{Tb}^{3+}\right]$ : [DNA] must be ca. 10:1 for fast ligation reaction. It is likely that notenough $\mathrm{Tb}^{3+}$ ion is available for binding to specific binding sites of the DNA-RNA complex when the $\left[\mathrm{Tb}^{3+}\right]$ : [DNA] ratio is below 10 .

Different hypotheses could be reasoned for reduced ligation yield at inhibitory $\mathrm{Tb}^{3+}$ concentrations. We showed hydrolysis and inactivation of the triphosphate of the donor RNA is not the cause of the inhibition. Although decomposition of the triphosphorylated RNA to mono- and diphosphorylated RNA was slightly enhanced in the presence of $200 \mu \mathrm{M} \mathrm{Tb}^{3+}$, more than 70 percent of donor RNA remained intact and triphosphorylated but not ligated. Hydrolysis, site specific degradation of the branched 
product or any evidence for increased reverse reaction was not observed in the presence of $\mathrm{Tb}^{3+}$ and in the presence or absence of inorganic pyrophosphate. Two other yet possible hypotheses for inhibition at high lanthanide concentrations are: 1) Misfolding of DNA to an inactivated form in the presence of high concentrations of $\mathrm{Ln}^{3+}$. 2) Displacement of the active bivalent metal ion from (a) catalytically important position(s) by $\mathrm{Ln}^{3+}$.

Combinatorial mutation interference analyses led to minimization of 9F7 and 9F13 catalytic cores to $70 \%$ and $57 \%$ of original lengths. The trimmed 9F7 contained 27 nucleotide in the catalytic core from which 11 nucleotides were guanines. Trimmed 9F13 was 23 nucleotide long with 12 guanines in the catalytic core. Trimmed 9F7 was slightly slower compared to the original length 9F7 when performing the reaction in the presence of $2 \mathrm{mM} \mathrm{Mn}^{2+}$ or $80 \mathrm{mM} \mathrm{Mg}^{2+}$. But importantly the trimmed and original 9F7 were equally fast in the presence of optimum $\mathrm{Tb}^{3+}$. Trimmed version of 9F13 was 4 fold faster than original length enzyme. It can be deduced that the full length 9F13 had inactive folds for which the mutable region was participating. The removal of this region eliminates possibility of formation of inactive folds of the enzyme.

For CoMA of 9F7, several 2'-OH sensitive positions were observed. Presence of high concentration of bivalent metal ion or optimum concentrations of terbium had relieved detrimental effects of 2'-OH. The relief of the 2'-OH effect was a significant observation especially when considering the reaction times of the CoMA separation steps. The $9 \mathrm{~F} 7$ reaction at low $\mathrm{Mn}^{2+}$ and $\mathrm{Mg}^{2+}$ alone were stopped after 8-10 h, while at high $\mathrm{Mn}^{2+}$ and low $\mathrm{Mn}^{2+}+\mathrm{Tb}^{3+}$ and $\mathrm{Mg}^{2+}+\mathrm{Tb}^{3+}$ the reaction times were $20 \mathrm{~min}, 1 \mathrm{~h}$ and $2 \mathrm{~h}$.

Performing CoMA at various conditions allowed comparison of the nucleobases requirement of $\mathrm{Mg}^{2+}$ and $\mathrm{Mn}^{2+}$ dependent reactions of 9F7. In general nucleobase requirement of 9F7 was higher for $\mathrm{Mg}^{2+}$ dependent reaction. Mutations were better tolerated at elevated concentrations of metal ions or in the presence of optimum terbium. Additionally, nucleotides G41 and T42 were included in the mutation analysis as positive controls, for which the nucleotide requirements were known from previous studies. ${ }^{149}$ These two nucleotides interact with the acceptor substrate upstream of the branch-site nucleotide. Disruption of the DNA-RNA Watson-Crick base-pairs inhibited the reaction. ${ }^{149}$ The CoMA results confirmed these interactions, since only the parent (ribo) nucleotides were tolerated, but all mutations showed large interference effects under all assayed conditions.

$\mathrm{Tb}^{3+}$ luminescence of folded 9F7 in pre-catalytic state in presence of $\mathrm{Mg}^{2+}$ or $\mathrm{Mn}^{2+}$ was studied. As it was expected in the presence of elevated concentrations of bivalent metal ions the $\left[\mathrm{Tb}^{3+}\right]_{1 / 2}$ was increased. Comparison of luminescence binding curves of an inactive mutant of the enzyme (G4T) and the original enzyme showed a left-shift, indicating facilitated binding of $\mathrm{Tb}^{3+}$ when the active site is not intact. This observation was more pronounced at low concentrations i.e. for transition from state-1 to state-2, in $\mathrm{Tb}^{3+}$ titration to the folded enzyme in presence of $\mathrm{Mg}^{2+}$. These results underline the finding that coordination of a divalent metal ion in the active site is essential for activity, and further support the 
interpretation that $\mathrm{Tb}^{3+}$ first binds to peripheral sites before displacing catalytic metal ions, and causing inactive conformations. However, since the luminescence data were all recorded with inactivated substrates and reported on the sum of specific and unspecific binding of $\mathrm{Tb}^{3+}$ to the nucleic acid complex, the luminescence binding curves cannot be directly correlated to the kinetic results.

Although the luminescence data are not sufficient to fully describe the details of $\mathrm{Tb}^{3+}$ binding, the results support our kinetic data which suggested that the 9F7 deoxyribozyme provides specific binding sites for $\mathrm{Tb}^{3+}$ ions. We hypothesize that acceleration of the ligation activity results from $\mathrm{Tb}^{3+}$ binding to structurally important sites which causes an increased affinity for divalent metal ions at catalytically critical $\mathrm{M}^{2+}$ binding sites.

DMS Footprinting assay of 9F7 were in agreement with DNase I probing of the enzyme. Accessibility of the $\mathrm{N}^{7}$ of guanosines for methylation with DMS and the DNA backbone for cleavage with DNase I were generally reduced in post-hybridization states compared to pre-hybridization state as well as in post catalytic state compared to the pre-catalytic state. Presence of $\mathrm{Mn}^{2+}$ in post catalytic state of the enzyme reduced the DNase I and DMS based cleavage. The effect was more significant in DNase I probing assay.

Replacement of the $\gamma$-phosphate by PS in standard donor RNA caused a considerably reduced ligation rate. $\mathrm{Tb}^{3+}$ did not improve the reaction rate in this case. These results indicated that the larger and softer sulfur atom was less well accommodated than oxygen at the $\gamma$ position, and lent support to the hypothesis of preferred interaction of the terminal phosphate with hard lanthanides. Softer and more thiophilic metal ions such as $\mathrm{Cd}^{2+}$ and $\mathrm{Mn}^{2+}$ did not significantly rescue the PS effect.

The alternative donor substrate, "1G" donor, was less efficiently ligated when triphosphorylated. Ligation was inhibited with $\gamma$ - phosphorothioate, as in standard donor. In contrast, with $\alpha$ phosphorothioate, the reaction rate was improved. Addition of $1 \mathrm{mM} \mathrm{Cd}^{2+}$ improved the reaction rate further when the reaction was performed in the presence of $20 \mathrm{mM} \mathrm{Mn}^{2+}$ while $\mathrm{Cd}^{2+}$ had no effect on tirphosphorylated "1G" substrate. Addition of $10 \mu \mathrm{M} \mathrm{Tb}^{3+}$ accelerated the PO substrate more than $\alpha$-PS substrate. Collectively, these results point towards important interactions at the $\alpha$ and $\gamma$-phosphates of the donor RNA, although the PS and rescue effects were less pronounced than for other metalloribozymes. ${ }^{150}$

The acceleration effect was observed for other deoxyribozymes which form 2',5' branched-RNA. This observation points toward favorable activation of the 2'-OH nucleophiles. It is conceivable that activating metal ions interact simultaneously with nucleophile and electrophile. Impressive rate enhancement and Increased affinity of the enzyme to bivalent metal ions allowed branch formation at $\mathrm{Mg}^{2+}$ concentrations nearer to the $\mathrm{Mg}^{2+}$ concentrations in biological systems ${ }^{99}$. In summary, these 
finding shifted the reaction rates and metal ion requirement of the enzyme into a region that makes it practical for applications in vivo and in vitro e.g. RNA engineering. 


\subsection{BX22, a lariat forming deoxyribozyme}

In addition to 9F7 deoxyribozyme family and three-helix junction forming deoxyribozymes, we were interested to investigate the catalytic mechanism and nucleotide requirements of another type of 2',5'branched RNA forming deoxyribozymes which is capable of formation of biological lariat RNAs. 6BX22 catalyzes the nucleophilic attack of the 2'-OH of a specified branch site at the acceptor RNA to the alpha phosphate of a triphosphorylated donor RNA. Pyrophosphate is released and the 2',5' phosphodiester bond is formed. $6 \mathrm{BX} 22^{13}$ was selected in vitro, to ligate RNA sequences with natural sequence of spliceosome substrates. The in vitro selection started with a DNA library containing a 40 nucleotide random region embedded with two constant binding arms to bind to the donor RNA (5' of the random region) and acceptor RNA (3’ of the random region).

The outcome of the selection, 6BX22 had 39 nucleotide in the catalytic core ${ }^{13}$ and was predicted to form a stem loop at the 3 ' end of the catalytic core ${ }^{26}$. The in vitro selection was performed in presence of 20 $\mathrm{mM} \mathrm{Mn}^{2+}$. It was shown that the enzyme is not active with any of the followed metal ions: $\mathrm{Mg}^{2+}, \mathrm{Ca}^{2+}$, $\mathrm{Zn}^{2+}, \mathrm{Fe}^{2+}, \mathrm{Co}^{2+}, \mathrm{Ni}^{2+}, \mathrm{Cd}^{2+},\left[\mathrm{Co}\left(\mathrm{NH}_{3}\right)_{6}\right]^{3+}$ when provided as chloride salts in concentrations ranging between $10 \mu \mathrm{M}-10 \mathrm{mM}^{26}$. 6BX22 was selected with A as branch site nucleotide but it was shown that the branch site can be mutated to $\mathrm{C}$ or $\mathrm{U}$ with almost similar activity. Branch site $\mathrm{G}$ was accepted with dramatically reduced yield. Capability of lariat formation was maintained upon mutation to $\mathrm{C}$ and $\mathrm{U}$.

Formation of lariat RNAs with two yeast RNAs, YBL059W (69 nucleotides) and ACT1 (309 nucleotides) and one human related RNA fragment, ß-globin IVS1 (130 nucleotides) with 6BX22 enzymes was proved to be efficient. In all three cases, the triphosphorylated RNAs were prepared by in vitro transcription with T7 RNA polymerase. The loop size of the lariat products of YBL059W, ACT1 and ß-globin were 51, 266 and 94 nucleotides respectively. Preparative (nanomole) scale synthesis of ACT1 lariat RNA was shown to be successful, however disruptor DNAs were required to prevent RNA degradation $^{26}$.

\subsubsection{BX22 substrate requirements}

Our initial step for functional analysis of 6BX22 was combinatorial mutation interference analysis (CoMA). One of the requirements of CoMA is ligation of one of the substrates to the mutant DNA libraries which topologically cannot be accommodated by a lariat RNA. Therefore, in this study, intermolecular 2',5' branch formation, employing distinct acceptor and donor RNA substrates were studied rather than lariat RNA formation. Firstly, we optimized the length of the donor RNA for the bimolecular format reaction which is required for the second step of CoMA (Fig 2.2-1 B). 

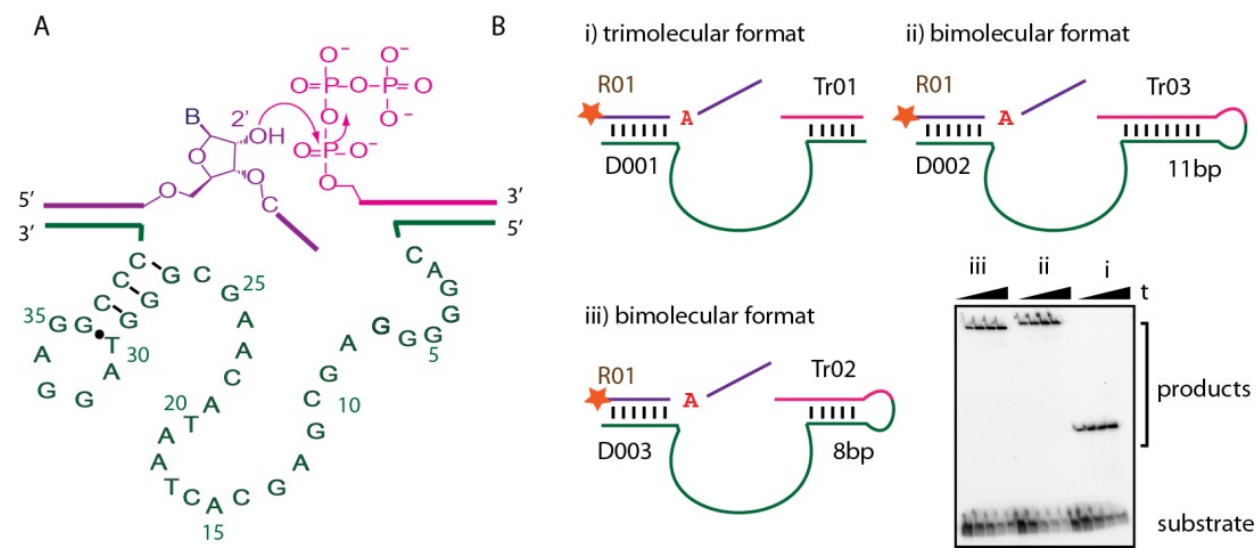

Fig 2.3-1 6BX22 predicted secondary structure and kinetics. A) mfold predicted secondary structure of 6BX22 and the catalyzed reaction by 6BX22. B) Optimization of 6BX22 binding arms length for trimolecular and bimolecular format

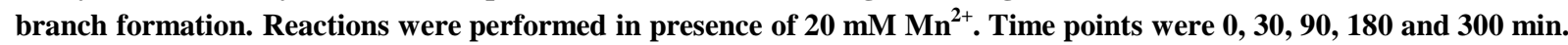

To perform bimolecular format reaction we chose the deoxyribozyme D003 with a binding arm with 8 nucleotides for base pairing with Tr02. This length of base pairing is not sufficient to perform the reaction in trimolecular format. For trimolecular format kinetic experiments performed for 6BX22 Tr03 was employed as donor RNA, forming 11 nucleotide long base paired region with the enzyme right binding arm, unless otherwise mentioned.

Additionally, we explored 6BX22 substrate requirement, regarding the acceptor RNA and the length of the RNA overhang after the branch site. 6BX22 was shown to accept up to 40 nucleotide overhang (ACT1 lariat formation) ${ }^{26}$. Here the minimal length of the overhang required for the catalysis was investigated. The standard acceptor RNA which is used in this study is R01 with 9 nucleotide overhang after the branch site. Three minimized RNAs which form either none or 1 or 3 nucleotide overhangs have been tested. 6BX22 was not capable of ligation when the adenosine branch site is the 3' end of the acceptor RNA i.e. at least one nucleotide overhang was shown to be required. Presence of 3 nucleotide overhang increased the reaction rate even higher than the original substrate which has 9 nucleotides overhang. (Fig 2.3-2) The results suggested that the first nucleotide downstream of adenosine branch site (in this study, a cytosine) is required for catalysis.

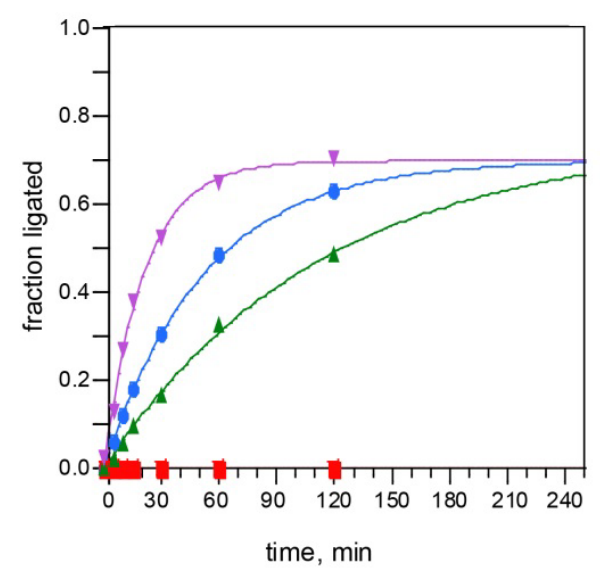

Fig 2.3-2 Kinetic assays for minimization of 6BX22 acceptor RNA. The four acceptor RNAs used here have identical sequences 5' of the branch site, therefore one deoxyribozyme (D002) was appropriate for all four acceptor RNAs. R02 did not have any nucleotide overhang after the branch site adenosine. R03 and R04 beared one or three nucleotides after the branch site. R01 was the original acceptor substrate for 6BX22. The sequences 3' of the branch site are shown on the right. The adenosine branch site is underlined. 


\subsubsection{Combinatorial mutation interference analysis of 6BX22}

To identify the catalytically important nucleotides of the 6BX22 enzymatic core, combinatorial mutation interference analysis (CoMA, section 2.1) was performed. For the synthesis of the mutant libraries, the $\mathrm{rN} / \mathrm{dN}$ mixtures were prepared by mixing of $100 \mathrm{mM} \mathrm{rN}$ and $\mathrm{dN}$ phosphoramidite solutions with the ratio of 10:90. Before synthesis of the mutant libraries the quality of the mixtures and the efficiency of the $\mathrm{rN}$ incorporation were analyzed by synthesis of analytical pentamers with the sequence of $\operatorname{dTdT}(\mathrm{rN} / \mathrm{dN}) \mathrm{dTdT}$. After synthesis and basic deprotection, the analytical samples were injected on anion exchange HPLC. Pentamers which contained the mutant rN had the 2'-O-TOM group and therefore their retention times were increased compared to the pentamers which contained the parent $\mathrm{dN}$. The integrated area under both $\mathrm{UV}^{260}$ peaks for dTdTdNdTdT and dTdTrNdTdT were extracted and the efficiency of $\mathrm{rN}$ incorporation was calculated. The result is summarized in table 2.3-1.

Table 2.3-1 Efficiency of $\mathrm{rN}$ incorporation in a mixture of $\mathrm{rN}: \mathrm{dN}$ phosphoramidites 10:90. Data were obtained experimentally for TT(rN/dN)TT pentamers. For details on synthesis and analysis procedure see text.

\begin{tabular}{lllll}
$\begin{array}{c}\text { Parent } \\
(\mathrm{dN})\end{array}$ & $\begin{array}{l}\mathrm{rA} \\
(\%)\end{array}$ & $\begin{array}{l}\mathrm{rC} \\
(\%)\end{array}$ & $\begin{array}{l}\mathrm{rG} \\
(\%)\end{array}$ & $\begin{array}{l}\mathrm{rU} \\
(\%)\end{array}$ \\
\hline $\mathrm{A}$ & 7.7 & 4.3 & 7.7 & 5.5 \\
$\mathrm{C}$ & 8.8 & 5.9 & 7.1 & 9.1 \\
$\mathrm{G}$ & 8.1 & 6.5 & 8.4 & 6.7 \\
$\mathrm{~T}$ & 8.5 & 6.3 & 6.6 & 6.9
\end{tabular}

After synthesis, deprotection and PAGE purification of the four mutant DNA libraries, donor RNA (Tr02) was ligated to the mutant libraries using T4 RNA ligase. The donor RNA-DNA libraries were radiolabeled at 3' end. The radiolabeled donor RNA-DNA libraries were subjected for the 6BX22 mediated branch formation with slight excess of acceptor RNA (R01). Active fractions were separated by denaturing PAGE. Alkaline hydrolysis was performed and PAGE analysis revealed the present mutants in each fraction of each library.

Separation of active mutants and the conditions in which this separation takes place is a crucial step in CoMA. Short incubation times exclude the slow mutants being present in the active fraction, whereas too long incubation times allow presence of very slow mutants. For one of the 6BX22 libraries, rA mutant library, separation of active mutants was performed after different ligation times in presence of $20 \mathrm{mM} \mathrm{Mn}^{2+}$ (Fig 2.3-3) The hydrolysis patterns for 0.5, 2, 3 and 5h were quite similar except for the band A31 which only appeared after 2h. Presence of the 2'-OH chemical tag in position A31 caused an interference value above 2.0 when $6 \mathrm{BX} 22$ reaction was stopped at $30 \mathrm{~min}$ which was reduced at longer time points. Therefore, we concluded that presence of 2'OH tag at position A31 reduces the rate of 6BX22 reaction but is not detrimental. For the rest of the libraries, we chose to perform separation step of CoMA by $3 \mathrm{~h}$ of incubation. 


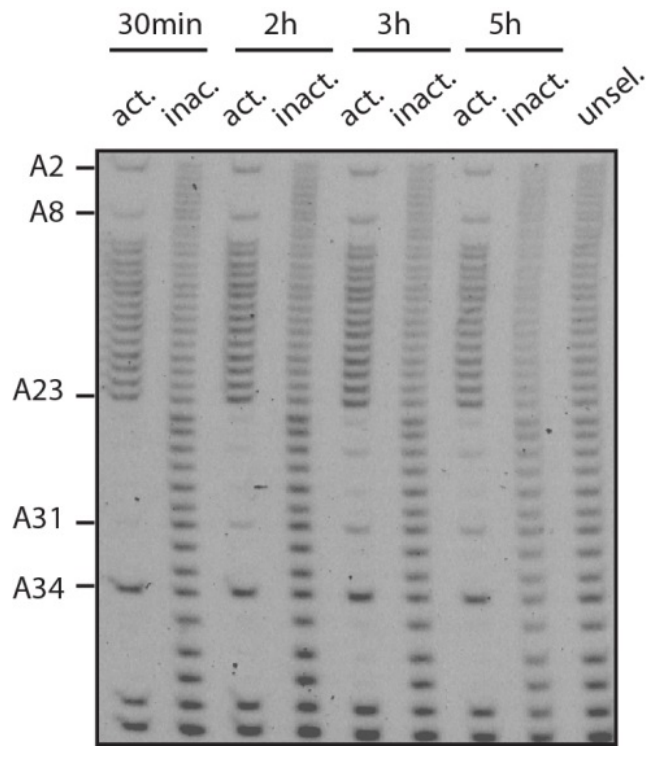

Fig 2.3-3 Kinetics of CoMA. rA mutant library of 6BX22 was subjected for CoMA with different ligation times. The reaction time for 6BX22 catalysis in step 2 of CoMA was limited to 0.5, 2, 3 and 5 hs. The hydrolysis patterns were almost similar for most bands except for A31. Data suggests that presence of the 2'-OH at A31 reduces the reaction rate but is not detrimental.

Fig 2.3-4 shows the CoMA of 6BX22 for all four libraries. The interference values are depicted for each library and each position. In CoMA, interference values for positions in which the 2'-OH chemical tag itself is detrimental are not informative. It is conceivable that those positions are sensitive to the sugar and DNA backbone conformation. In 6BX22 catalytic core, there are six examples of such positions; G3, G5, G6, C26, G28, G32 (numbers are based on original 6BX22 catalytic core).

The nucleotides in between G11-A24 accepted all the three possible mutations under the experimental condition, suggesting that this region is not involved in formation of the catalytic core. We hypothesized that the catalytic core of 6BX22 consists of two subdomains, upstream and downstream of the mutable region and the mutable region is responsible only as a linker.

This hypothesis was proven by kinetic experiments on minimized versions of 6BX22 enzyme. Minimization kinetics showed that although the identity of the linker sequence was not important, the length of the linker played a crucial role and must be longer than one nucleotide. The optimum length for the linker was observed to be 2 nucleotides. The trimmed version in which the linker region was totally exchanged by two thymidines was chosen as the trimmed 6BX22 for further experiments of this sub chapter. Formation of branched product with the trimmed 6BX22 in standard condition has the $k_{\text {obs }}$ of $0.01-0.03 \mathrm{~min}^{-1}$ and reaches ca $80 \%$ in $3 \mathrm{~h}$. The capability of the enzyme for lariat formation was maintained to the same extent after minimization. (Fig 2.3-5). For further experiments the nucleotide numbers refer to the number of that specific nucleotide in the trimmed version (except if mentioned otherwise). The nucleobases having different numbers before and after trimming are marked with superscript " $t$ " as an abbreviation for the word "trimmed". 


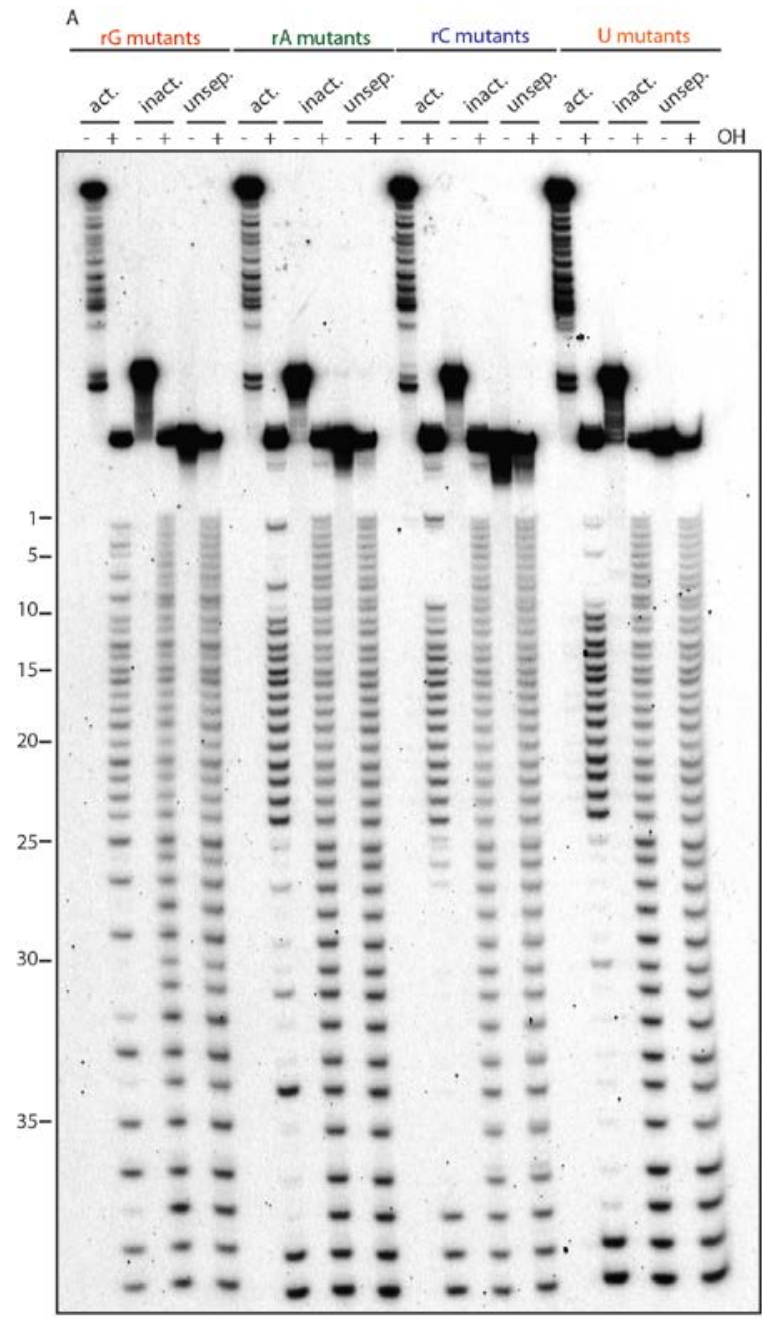

B
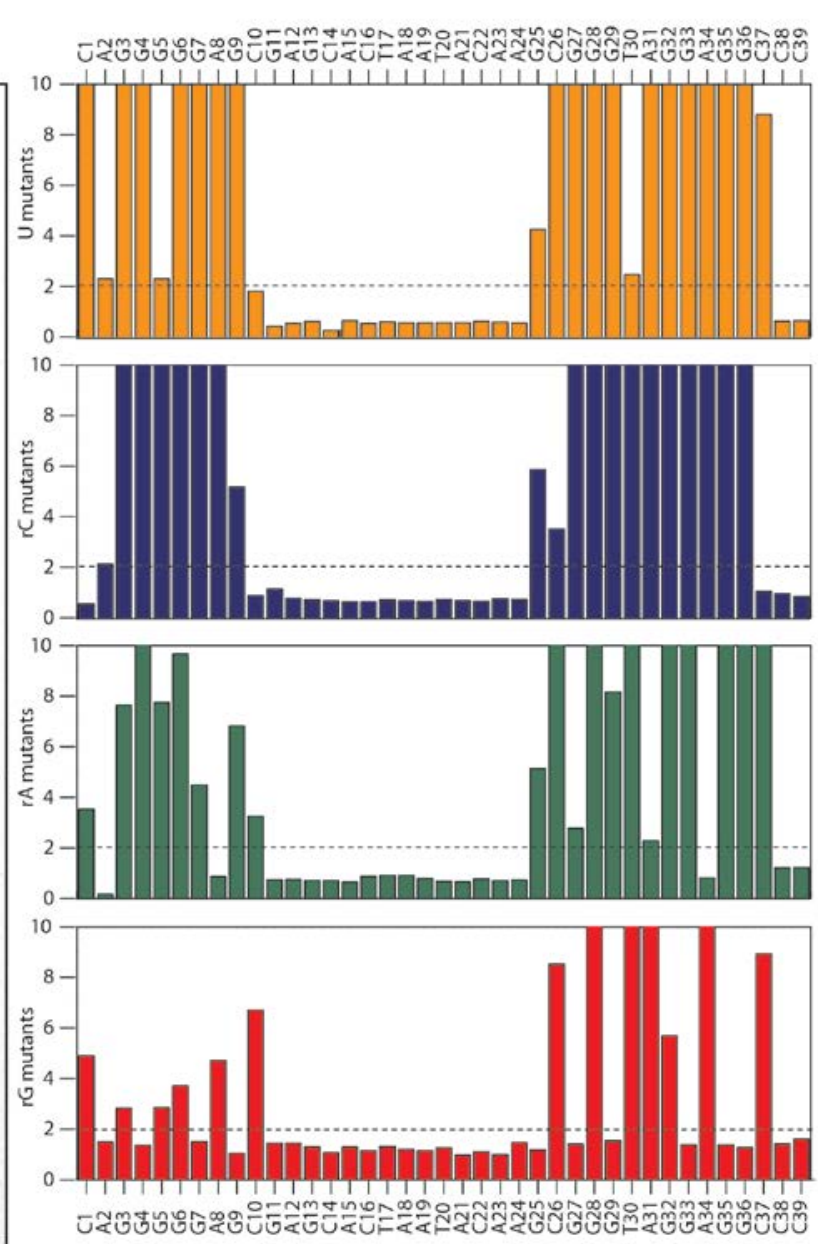

$6 \mathrm{~B} \times 22$ Core

Fig 2.3-4 Combinatorial mutation interference analysis (CoMA) for 6BX22. The separation step of CoMA (the second step) was performed after 6BX22 ligation in presence of $50 \mathrm{mM}$ HEPES pH 7.5, $150 \mathrm{mM} \mathrm{NaCl}, 2 \mathrm{mM} \mathrm{KCl,} 20 \mathrm{mM}$ $\mathrm{MnCl}_{2}$ at $37^{\circ} \mathrm{C}$. A) Autoradiogram of the hydrolysis gel of active, inactive and unselected libraries of $6 \mathrm{BX} 22$. B) Interference values of 6BX22 mutant libraries. Interference values were calculated by the ratio of intensity of a band in the unseparated library over the intensity of the corresponding band in the active fraction. Nucleotide numbers are based on original 6BX22 core.

For some of the positions in which the 2'-OH tag was detrimental, we performed kinetic experiments with point mutants of trimmed 6BX22, to get insights about mutation interference (Fig 2.3-6). Mutation of G3, G6, G16 and G20 to A (the last two positions, G16 ${ }^{t}$ and G20 ${ }^{t}$, are numbered as G28 and G32 in original catalytic core of the enzyme), resulted in either inactivation or reduced activity of the mutant. Mutations of $\mathrm{G}^{\mathrm{t}}{ }^{\mathrm{t}}$ and $\mathrm{G} 20^{\mathrm{t}}$ to $\mathrm{C}$ and $\mathrm{T}$ totally aborted the enzymatic activity of the enzyme. Altogether, these results suggest that at positions in which the DNA backbone conformation was a determining factor, the identity of the nucleobases is also crucial for catalysis. The only active point mutant was G20 mutated to A with reduced activity i.e. 20\% yield after $3 \mathrm{~h}$. 
A

6BX22 ori. CAGGGGGAGCGAGCACTAATACAAGCGGGTAGGAGGCCC

6BX22 trim. CAGGGGGAGCTTGCGGGTAGGAGGCCC

6BX22 trim+1 CAGGGGGAGCGGCGGGTAGGAGGCCC

6BX22 trim+8 CAGGGGGAGCGAGCAAGCGGGTAGGAGGCCC
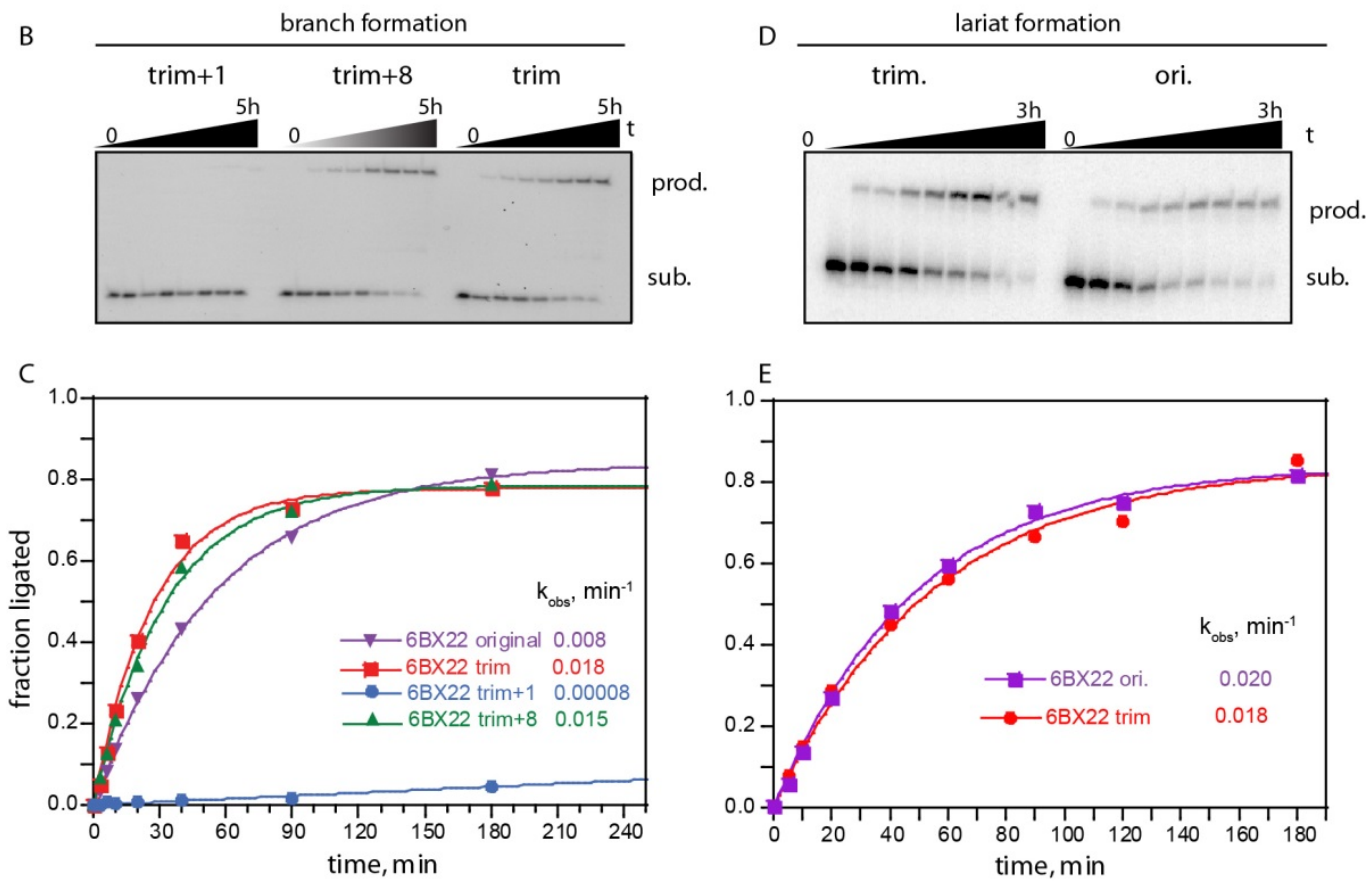

Fig 2.3-5 6BX22 trimming kinetics. A) 6BX22 original and trimmed enzymatic cores. The region which accepts all mutations and apparently does not participate in formation of catalytic core is marked green and underlined. The replacement TT in trimmed version of 6BX22 (marked red) was not originally present in the sequence. B) Branch formation catalyzed by trimmed 6BX22 with standard substrates. Time points were:0,1, 5, 10, 20, 40, 90, 180 min. C) Kinetic curves related to B. D) Lariat formation with original and minimized 6BX22. YLB059W mRNA (Tr04) was used as substrate. Time points were the same as B with an additional time pint at $120 \mathrm{~min}$. E) kinetic curves related to D.

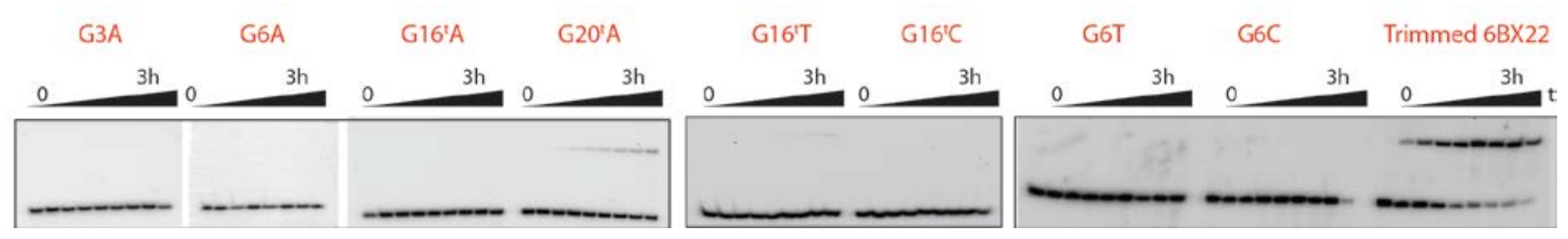

Fig 2.3-6 Point mutant kinetics of trimmed 6BX22. Point mutants were directing positions at which the 2'-OH was detrimental. Kinetic experiments were performed with point mutation on trimmed 6BX22, except for G6A which is performed with a mutation on original $6 \mathrm{BX} 22$. Time points were $0,5,10,20,40,60,90,120,180$. All the mutants were inactive except G20A with $20 \%$ yield at $3 \mathrm{~h}$. The numbers are based on the position of the specific nucleotides in the trimmed 6BX22 catalytic core. The positions which have different numbers after trimming are marked with superscript “t”.

The essential sequence of 6BX22 was shown to be 27 nucleotide long and contain 14 guanosines. High G-content of the catalytic core of the 6BX22 enzyme suggested presence of a G-quadruplex. The minimal requirement for formation of a G-quadruplex is four times 2 guanosines in a row, which is present in the minimized 6BX22 catalytic core. In this study, this hypothesis was investigated by studies on metal ion dependence of the enzyme, foot printing assays, spectroscopic methods and DNA nucleotide analog interference mapping $\left(\mathrm{dNAIM}^{140}\right)$ which are described in the next sections. 


\subsubsection{BX22 dNAIM and DMS-interference}

The high G-content of 6BX22 core suggested presence of a G-quadruplex in its structure. Minimally two stacking G-quartets are required for formation of a G-quadruplex. The guanines participating in formation of a G-quartet employ functional groups at both Watson-Crick and Hoogsteen sides to form hydrogen bonds. Here we aim to study the functional group requirements of the guanines of the trimmed 6BX22 catalytic core by DNA nucleotide analog interference mapping (dNAIM) ${ }^{140}$ and DMSinterference (DMSi) $)^{39}$.

dNAIM was developed in our research group and has conceptual similarities to CoMA. dNAIM consists of four steps; 1) synthesis of DNA libraries with the desired nucleobase modifications, 2) separation of active modified enzymes, 3) alkaline hydrolysis and 4) PAGE analysis. As in CoMA, the nucleobase modifications are statistically dispersed in the catalytic sequence and are encoded by a 2'-OH chemical tag.

For dNAIM of 6BX22, trimmed DNAs with G modifications were synthesized by solid phase synthesis using mixtures of modified ribo-G phosphoramidites with non-modified deoxyribo- $G$ phosphoramidite $\left(\mathrm{rG}^{\mathrm{mod}} / \mathrm{dG}\right)$. Mixture of $\mathrm{rG} / \mathrm{dG}$ was used to synthesize rG library as a control for the effect of 2'-OH chemical tag. The mixture $r-c^{7} G / d G$ was used to synthesis $c^{7} G$ (7deaza) library, revealing the importance of $\mathrm{N}^{7}$ of guanines. The two mixtures $\mathrm{r}$-2-aminopurine (2AP)/dG and r-m ${ }^{1} \mathrm{G} / \mathrm{dG}$ were used to synthesize libraries with modifications at Watson-Crick side of the guanines. The four modified libraries underwent the next steps of dNAIM including separation of active fractions, alkaline hydrolysis, PAGE and quantification.

For DMSi, non-modified trimmed 6BX22 DNA was subjected for methylation by DMS. DMS methylates $\mathrm{N}^{7}$ of guanosines. In addition to steric effects, methylated guanosines are charged positively at $\mathrm{N}^{7}$. The post synthetic DMS methylated library $\left(\mathrm{m}^{7} \mathrm{G}\right)$ was used in parallel to dNAIM libraries for separation of active species. Positions with detrimental effect of methylation were identifiable by piperidine cleavage and PAGE analysis. As DMSi was not dependent on alkaline hydrolysis and did not require the 2'-OH chemical tag, it provided data about guanines in which the 2'-OH tag was detrimental.

For dNAIM, 2'-OH tag resulted in interference values higher than threshold 2.0, at four positions G3, G6, G16 ${ }^{\mathrm{t}}$ and G20 ${ }^{\mathrm{t}}$, consistent with CoMA data. For these positions only the data from DMSi revealed information about the $\mathrm{N}^{7}$ of guanines. Interference of 2'-OH at G5 was at the border of being significant (interference values of 2.8 and 1.7 respectively for CoMA and dNAIM).

$c^{7} G$ dNAIM and DMSi reflect the necessity of the N7 position for formation of hydrogen bonds. The two libraries reveal complementary information. When a band is absent in both libraries one can deduce that the $\mathrm{N}^{7}$ is involved in direct metal ion coordination or formation of a hydrogen bond. DMS based $\mathrm{N}^{7}$ 
methylation resulted in interference values higher than 2 at G4, G5, G17 ${ }^{t}, G 20^{t}, G 21^{t}, G 23^{t}, G 24^{t}$ and at all the 2'-OH sensitive positions i.e. G3, G6, G16 ${ }^{t}$ and G20 ${ }^{t}$. For positions G4, G20 ${ }^{t}, \mathrm{G}^{\mathrm{t}}{ }^{\mathrm{t}}$ and $\mathrm{G} 24^{\mathrm{t}}$ the $\mathrm{N}^{7}$ methylation was not detrimental but reduced the band intensity of the active fraction and caused interference values between 2 and 5. Excluding the 2'-OH sensitive positions, $c^{7} G$ library revealed involvement of G4, G5 and G21 ${ }^{\mathrm{t}}$ for the catalysis. G9 showed interference values slightly above threshold. For most of the positions $c^{7} G$ data and DMSi data were similar except at four positions. G4 and $G 20^{t}$ had high interference value at $c^{7} G$ library but had less interference at DMSi analysis. Methylated $G 17^{t}$ and $G 23^{t}$ were detrimental while the absence of $\mathrm{N}^{7}$ in the $\mathrm{c}^{7} \mathrm{G}$ library was accepted.

Both $\mathrm{m}^{1} \mathrm{G}$ and 2AP libraries give insights about the Watson-Crick side of guanines. In the $\mathrm{m}^{1} \mathrm{G}$ library formation of Watson-Crick base pair is disturbed by steric effects. The library with 2aminopurin lacks the keto functional group at the position 6 of the guanine and therefore is able to form maximum two hydrogen bonds instead of three. Both libraries resulted high interference by modifications at all guanines except for G5 in $\mathrm{m}^{1} \mathrm{G}$ library, G7 in 2AP library and $\mathrm{G}^{\mathrm{t}}$ and $\mathrm{G} 15^{\mathrm{t}}$ in both libraries (Fig 2.37). In summary, dNAIM and DMSi, revealed which functional groups of the necessary guanosines are modifiable and are not involved in formation of any hydrogen bond or coordination to metal ions.
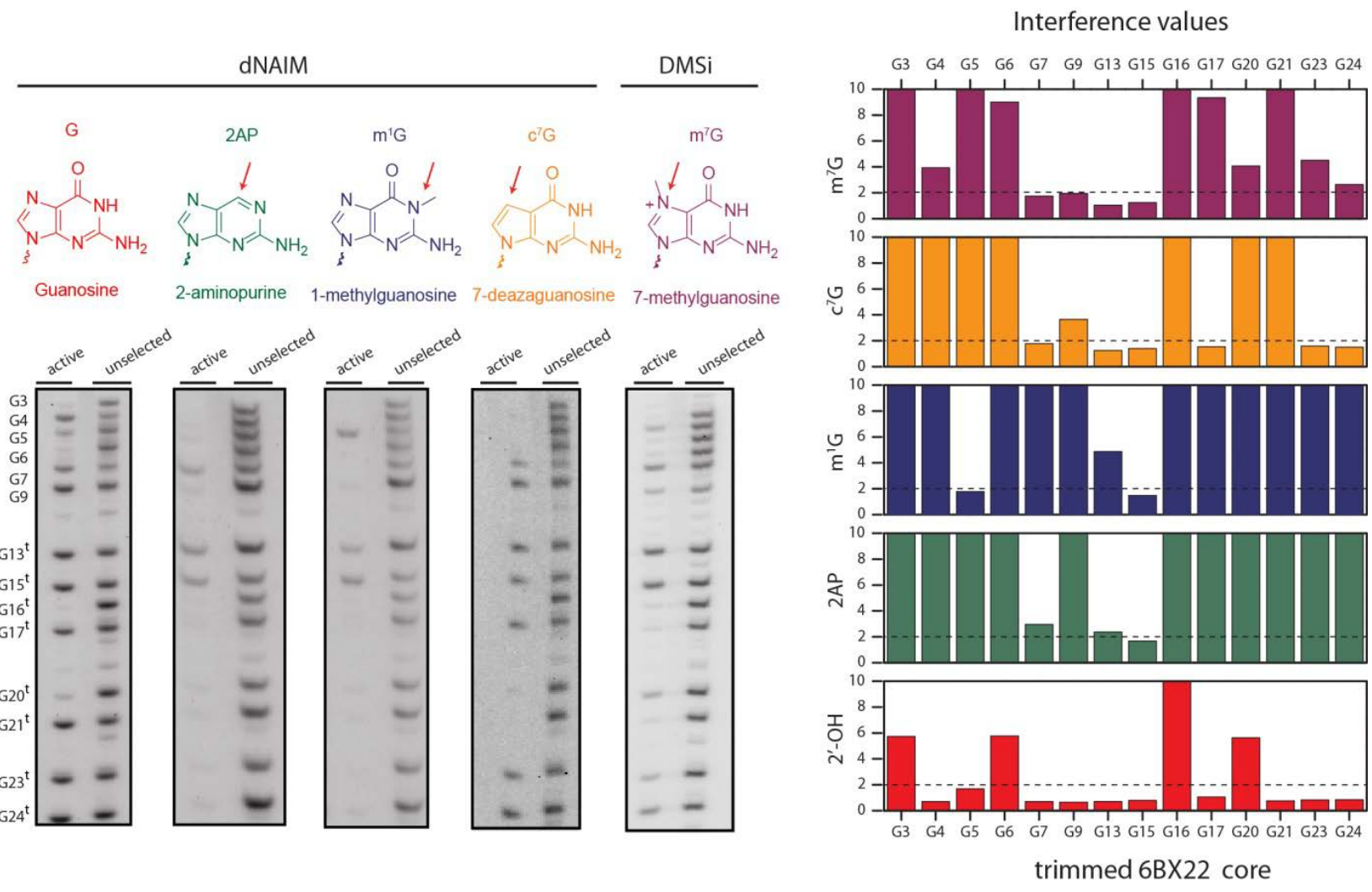

Fig 2.3-7 Nucleotide analog interference mapping of DNA (dNAIM) and DMS-interference (DMSi) of guanines of the trimmed 6BX22. Modifications of dNAIM libraries were incorporated to the 6BX22 sequence during solid phase synthesis. Modification of the DMSi library was post synthetic using DMS. 6BX22 reactions for separation of active modified DNAs were in presence of $50 \mathrm{mM}$ HEPES pH 7.5, $150 \mathrm{mM} \mathrm{NaCl}, 2 \mathrm{mM} \mathrm{KCl}$ and $20 \mathrm{mM} \mathrm{Mn}^{2+}$ at $37^{\circ} \mathrm{C}$ for $3 \mathrm{~h}$. Interference values were defined as in CoMA by the ratio of a band in the unselected library to the corresponding band in the active fraction. 


\subsubsection{BX22 and metal ions}

To further explore possibility of presence of G-quadruplex in the catalytic core of 6BX22 we were interested to study the dependence of the 6BX22 deoxyribozyme on metal ions. G-rich sequences are dependent on monovalent metal ions such as $\mathrm{Na}^{+}$or $\mathrm{K}^{+}$to form G-quadruplexes ${ }^{106}$. The quadruplex formation rate increases with increase in the concentration of these monovalent salts. ${ }^{151}$ Standard reaction buffer for $6 \mathrm{BX} 22$ contains $150 \mathrm{mM} \mathrm{NaCl}, 2 \mathrm{mM} \mathrm{KCl}$ and $20 \mathrm{mM} \mathrm{MnCl}_{2}$. If the catalytic activity of the enzyme is dependent on formation of a G-quadruplex, it is expected that it is reduced at lower monovalent salt concentrations. We performed kinetic experiments in the absence of $\mathrm{K}^{+}$ion or reduced amount or absence of $\mathrm{Na}^{+}$ion (in all cases in presence of $20 \mathrm{mM} \mathrm{Mn}^{2+}$ at $\mathrm{pH}$ 7.5). Although, the kinetics of the enzyme was reduced at lower monovalent concentrations but still the enzyme was active in the absence of both monovalent metal ions and reached almost $50 \%$ of the yield that it could achieve in the presence of standard concentration of monovalent metal ions (Fig 2.3-8). Taken together, these kinetic data did not support presence of G-quadruplex in the catalytic core of the enzyme.
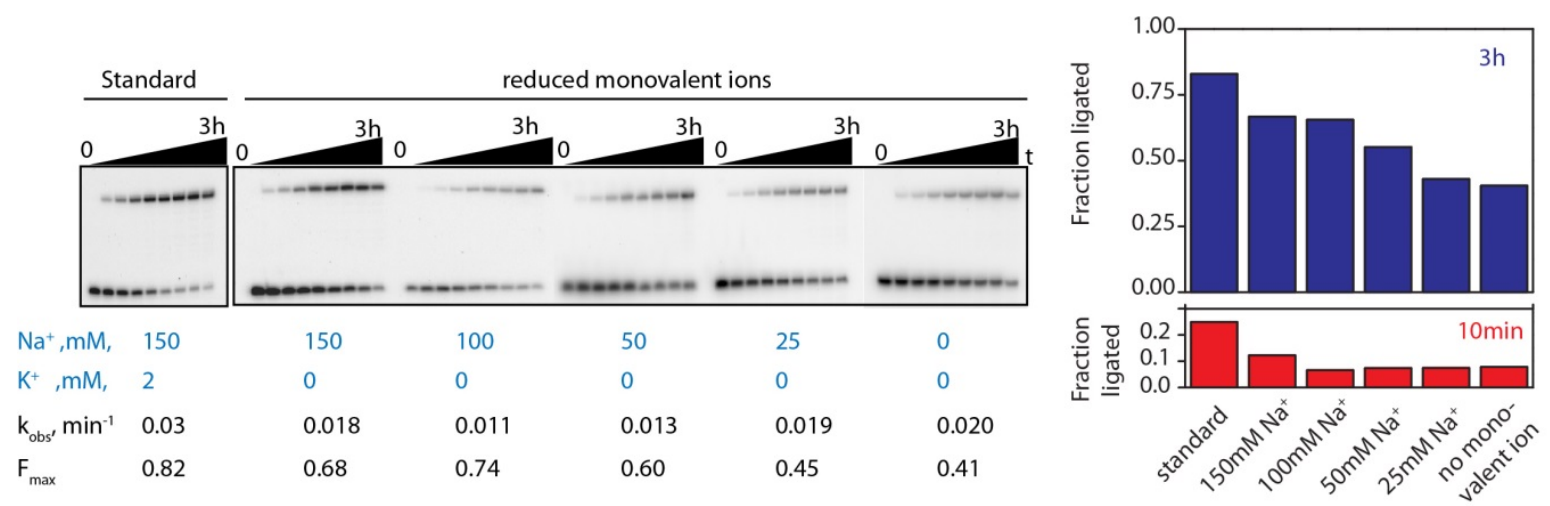

Fig 2.3-8 Kinetics of 6BX22 enzyme in presence of $20 \mathrm{mM} \mathrm{Mn}^{2+}$ and reduced concentrations of monovalent salts

Additionally, kinetic experiments have been performed to study the dependence of the enzyme on bivalent metal ions. In one experiment, the concentration of $\mathrm{Mn}^{2+}$ was reduced from $20 \mathrm{mM}$ to $0 \mathrm{mM}$. The total ionic strength was kept constant by addition of appropriate concentrations of $\mathrm{Mg}^{2+}$ to compensate reduced concentration of $\mathrm{Mn}^{2+}$. The gel in Fig 2.3-9 A shows the ligation efficiency after 3h for combinations of $\mathrm{Mg}^{2+}$ and $\mathrm{Mn}^{2+}$ concentrations. The results are plotted in the same figure $\mathrm{B}$. Additionally, product formation in the course of reaction was monitored in the presence of $5 \mathrm{mM} \mathrm{Mn}^{2+}$ and $15 \mathrm{mM} \mathrm{Mg}^{2+}$. The reaction rate was reduced 6-fold and reached $40 \%$ yield after $10 \mathrm{~h}$ incubation at $37^{\circ} \mathrm{C}$. 
A

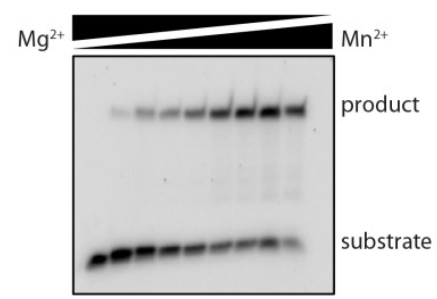

B

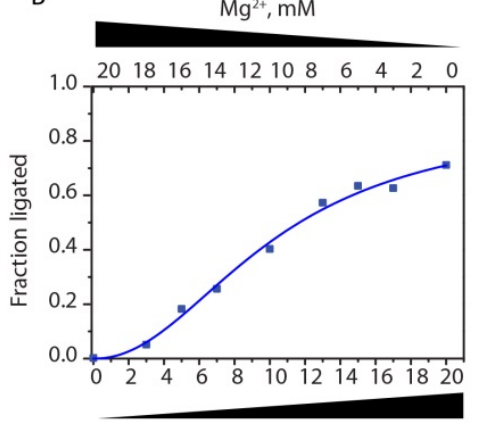

C

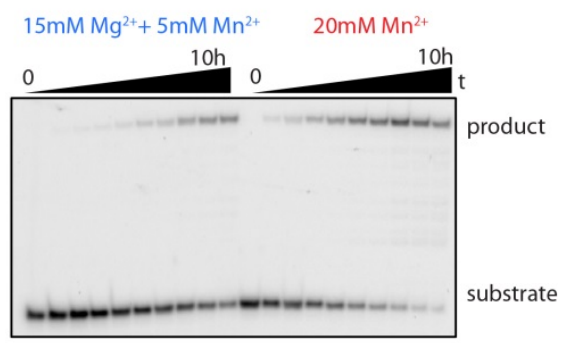

D

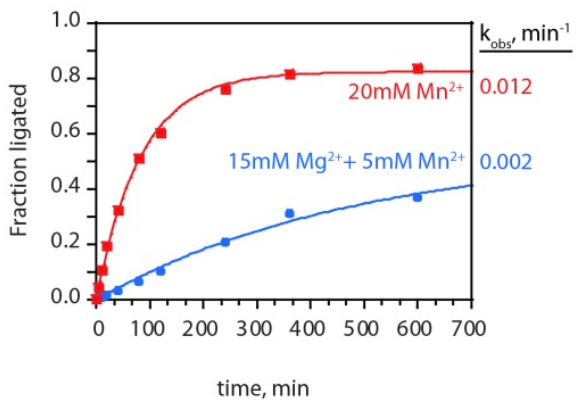

Fig 2.3-9 Kinetics of trimmed 6BX22 with reduced $\mathrm{Mn}^{2+}$. $\mathrm{A}$ and $\mathrm{B}$ ) ligated fraction after $3 \mathrm{~h}$ incubation at different combinations of $\mathrm{Mg}^{2+}$ and $\mathrm{Mn}^{2+}$. C and D) individual kinetic data in presence of $20 \mathrm{mM} \mathrm{Mn^{2+ }}$ and $15 \mathrm{mM} \mathrm{Mg}^{2+}$ plus 5 $\mathrm{mM} \mathrm{Mn}{ }^{2+}$. Time points were $0,5,10,20,40,80 \mathrm{~min}, 2,4,6$ and $10 \mathrm{~h}$.

The trivalent cation, $\mathrm{Tb}^{3+}$, was shown to interact with G-quadruplex forming oligonucleotides derived from Tetrahymena ${ }^{122}$ or human telomeric sequences ${ }^{152}$. Here we investigated the activity of the enzyme 6BX22 in the presence of $\mathrm{Tb}^{3+}$. In general, addition of $\mathrm{Tb}^{3+}$ to the $\mathrm{Mn}^{2+}$ dependent reactions of 6BX22 reduced the rate and maximum yield of the reactions. Fig 2.3-10 shows the titration of $\mathrm{Tb}^{3+}$ in presence of different concentrations of $\mathrm{Mn}^{2+}$. $\mathrm{Tb}^{3+}$ concentration below $50 \mu \mathrm{M}$ had slight effects on kinetics of the enzyme while concentrations above $100 \mu \mathrm{M}$ reduced the yield dramatically. Incubation of the samples in presence of $\mathrm{Tb}^{3+}$ concentrations higher than $300 \mu \mathrm{M}$ caused degradation of RNA substrate. The rate constant determined by exponential fit to the kinetic curves are marked beside each panel. One should note that comparison of $k_{\text {obs }}$ is only meaningful when the extrapolated maximum yields are in the same range. In the case of $\mathrm{Tb}^{3+}$ titration, the maximum yields are reduced and therefore, $k_{\mathrm{obs}}$ values are not directly comparable.

To further investigate, the $\mathrm{Tb}^{3+}$ mediated inhibition of 6BX22, single and double chase experiments have been performed (Fig 2.3-11). Single chase experiment started with $10 \mathrm{mM} \mathrm{Mn}^{2+}$. After $20 \mathrm{~min}$ of starting the reaction, $\mathrm{Tb}^{3+}(100 \mu \mathrm{M})$ was added to the reaction mixture. As it was expected the rate of product formation and the final yield were reduced upon addition of $\mathrm{Tb}^{3+}$ (50\% vs $75 \%$ yield at $5 \mathrm{~h}$, compared to $10 \mathrm{mM} \mathrm{Mn}^{2+}$ ). For the double chase experiment, extra $\mathrm{Mn}^{2+}$ was added to the reaction mixture to reach final concentrations of $20 \mathrm{mM} \mathrm{Mn}^{2+}$ and $100 \mu \mathrm{M} \mathrm{Tb}^{3+}$. The reaction which was decelerated by addition of $\mathrm{Tb}^{3+}$ in the first chase was retrieved and reaction reached to $75 \%$ yield within 3h after addition of extra $\mathrm{Mn}^{2+}$. These data shows that inhibition of $6 \mathrm{BX} 22$ by $\mathrm{Tb}^{3+}$ is a reversible inhibition and suggests that both $\mathrm{Mn}^{2+}$ and $\mathrm{Tb}^{3+}$ compete for the same position in the enzyme which is crucial for catalysis. 

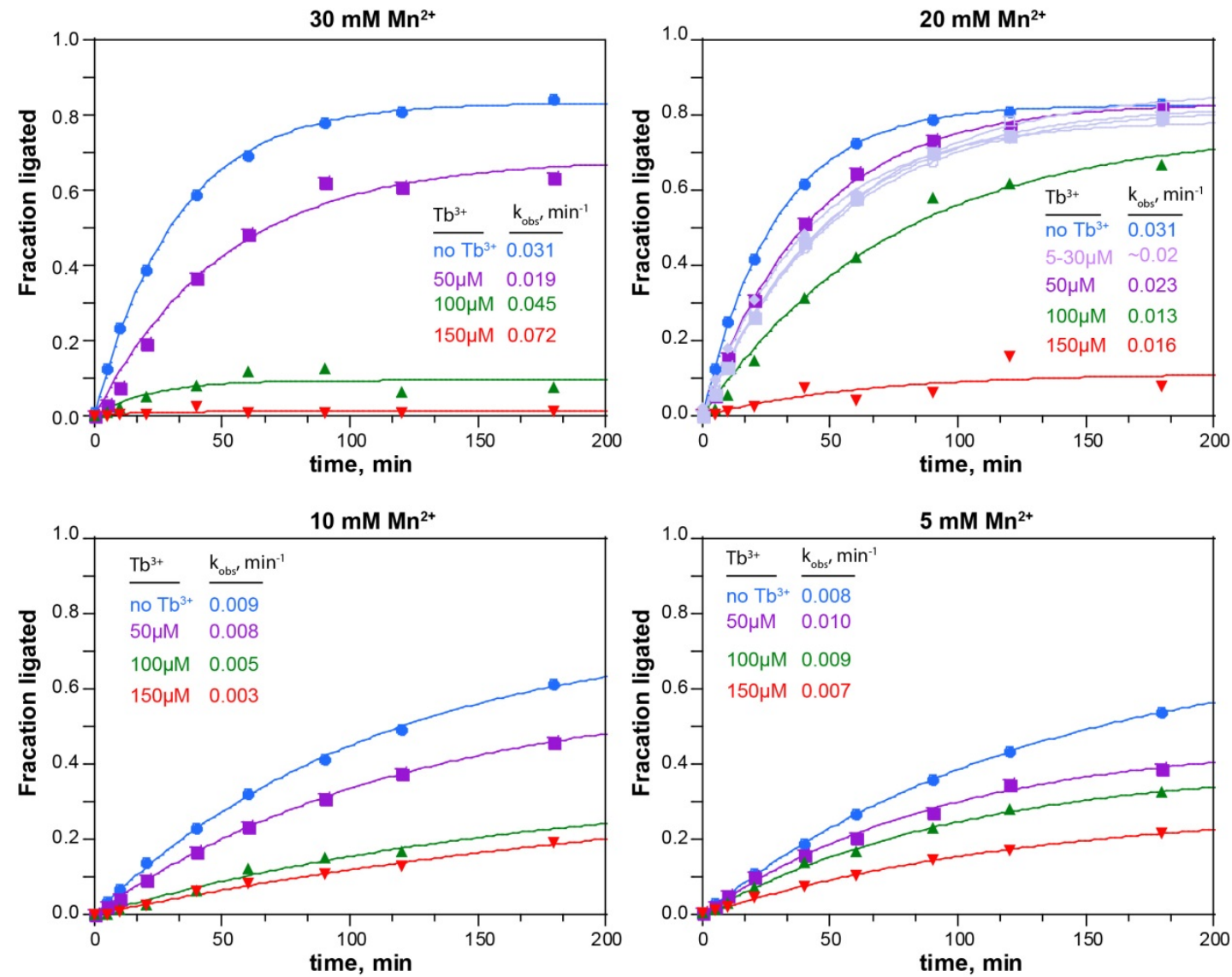

Fig 2.3-10 $\mathrm{Tb}^{3+}$ mediated inhibition of $\mathrm{Mn}^{2+}$ dependent $6 \mathrm{BX} 22$ reaction. The $\boldsymbol{k}_{\mathrm{obs}}$ values are not directly comparable as the extrapolated maximum yields are reduced upon presence of $\mathbf{T b}^{3+}$.

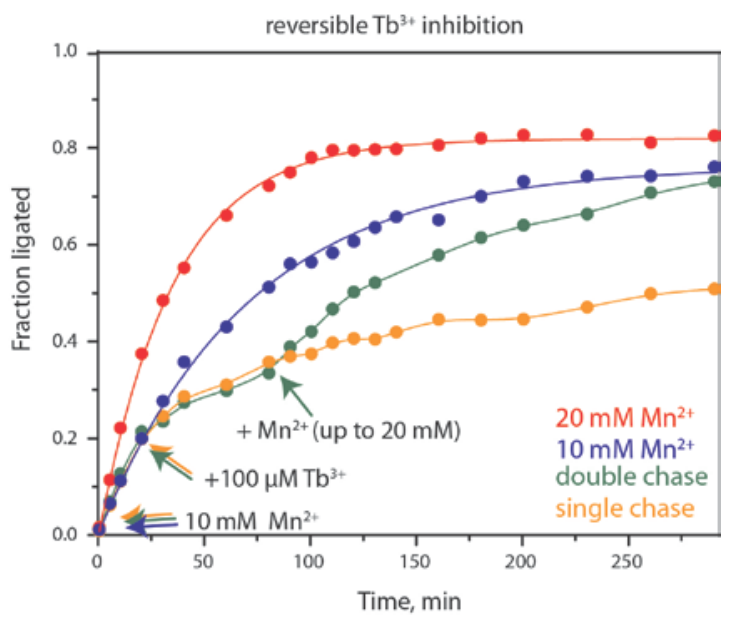

Fig 2.3-11 6BX22 single and double chase experiments with $\mathrm{Tb}^{3+}$ and $\mathrm{Mn}^{2+}$. For both chase experiments, addition of $\mathrm{TbCl}_{3}$ to the chase samples was $20 \mathrm{~min}$ after initiation of the reaction. For the double chase reaction, $\mathrm{MnCl}_{2}$ added to

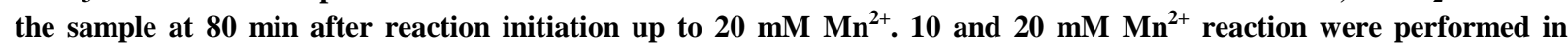
parallel as control experiments.

In summary, independence of the catalytic activity of 6BX22 from monovalent ions was in disagreement with presence of a G-quadruplex in its active structure. And reversible inhibition of the 
deoxyribozyme with $\mathrm{Tb}^{3+}$, showed there is (are) specific binding site(s) for $\mathrm{Tb}^{3+}$. Presence of such binding sites allowed luminescence studies of 6BX22.

\subsection{5 $\quad \mathrm{Tb}^{3+}$ luminescence}

Energy transfer from nucleic acids to inner-sphere coordinated $\mathrm{Tb}^{3+}$ ions is the basis of $\mathrm{Tb}^{3+}$ sensitized luminescence. This property makes $\mathrm{Tb}^{3+}$ an applicable probe for studying metal ion binding sites in DNA. $\mathrm{Tb}^{3+}$ luminescence was employed to study $\mathrm{Mg}^{2+}$ binding sites in ribozymes ${ }^{113}$ for which $\mathrm{Tb}^{3+}$ was an inhibitory metal ion ${ }^{148}$. In the case of $6 \mathrm{BX} 22$, the kinetic data suggested that $\mathrm{Tb}^{3+}$ binds to the same position of $\mathrm{Mn}^{2+}$ and causes reversible inhibition. Therefore, studying $\mathrm{Tb}^{3+}$ binding sites in $6 \mathrm{BX} 22$ would shed light on the hypothetical binding sites for $\mathrm{Mn}^{2+}$ as well.

Fig 2.3-12 A depicts excitation and emission spectra of $\mathrm{Tb}^{3+}$. For the excitation spectra the emission wavelength was set at $545 \mathrm{~nm}$ and excitation spectra of $150 \mu \mathrm{M} \mathrm{Tb}{ }^{3+}$ alone or in the presence of $2 \mu \mathrm{M}$ 6BX22 DNA (D010) was recorded. For the emission spectrum, $150 \mu \mathrm{M} \mathrm{Tb^{3+ }}$ in the presence of $2 \mu \mathrm{M}$ DNA (D010) was excited at $284 \mathrm{~nm}$. The four peaks, signature of $\mathrm{Tb}^{3+}$ sensitized luminescence, were detectable at 488, 543, 584 and $620 \mathrm{~nm}$. For $\mathrm{Tb}^{3+}$ titrations, the intensity of the peak at 543 was plotted against $\left[\mathrm{Tb}^{3+}\right]$.

$\mathrm{Tb}^{3+}$ titrations were performed with pre-annealed RNA-DNA complexes in the presence of $150 \mathrm{mM}$ $\mathrm{NaCl}, 2 \mathrm{mM} \mathrm{KCl}$ and 0 or $20 \mathrm{mM} \mathrm{MnCl}_{2}$. We were interested in the $\mathrm{Tb}^{3+}$ binding sites at the catalytic core of the enzyme. Hybridization of the enzyme binding arms to the RNA substrates or product leads to formation of stiff binding arms that can affect the three dimensional structure of the catalytic core of the enzyme. Therefore the DNAs used for $\mathrm{Tb}^{3+}$ titration were pre annealed with (inactivated) RNA substrates, “6BX22 hyb”. The acceptor substrate was the active substrate (R03) but the donor substrate was the inactive analog of Tr03 (R05) containing 5'-OH rather than 5'-triphosphate.

Fig 2.3-12 B shows the complexes that have been used in $\mathrm{Tb}^{3+}$ luminescence study of 6BX22. In addition to $\mathrm{Tb}^{3+}$ titration to $6 \mathrm{BX} 22 \mathrm{hyb}, \mathrm{Tb}^{3+}$ was titrated to left and right binding arms of the enzyme separately. This setup was aimed to specify how much each of the binding arms and the catalytic core contribute into the total intensity observed in $\mathrm{Tb}^{3+}$ titration to the $6 \mathrm{BX} 22$ hyb.

$\left[\mathrm{Tb}^{3+}\right]_{1 / 2}$ was observed to be $36 \mu \mathrm{M}$ with maximum intensity of ca 30 a.u. for the left binding arm, in the absence of $\mathrm{Mn}^{2+}$. $\left[\mathrm{Tb}^{3+}\right]_{1 / 2}$ was $139 \mu \mathrm{M}$ with maximum intensity of ca 50 a.u. for the right binding arm at the same conditions (also the same detector voltage). $\left[\mathrm{Tb}^{3+}\right]_{1 / 2}$ for $6 B X 22$ hyb was $110 \mu \mathrm{M}$ and reached maximum intensity of ca 100 a.u. Notably, the observed intensity for the left binding arm at concentrations below $100 \mu \mathrm{M} \mathrm{Tb^{3+ }}$ was higher in comparison to $6 B X 22$ hyb which consists of both binding arms and the catalytic core. This experimental setup showed that the $\mathrm{Tb}^{3+}$ luminescence data are not simply additive data and suggested that $\mathrm{Tb}^{3+}$ binding sites of the binding arms are affected when present in the context of the 6BX22 deoxyribozyme. 
In the presence of $20 \mathrm{mM} \mathrm{Mn}^{2+}$, as expected, the maximum luminescence intensity was decreased and $\left[\mathrm{Tb}^{3+}\right]_{1 / 2}$ was increased for the same construct; $\left[\mathrm{Tb}^{3+}\right]_{1 / 2}$ of $333 \mu \mathrm{M}$ and max intensity 25 a.u. for the active trimmed $6 B X 22$ hyb (at the same detector voltage as the previous experiment). Using an inactive form of trimmed 6BX22, containing a G6C mutation (D021), led to $\left[\mathrm{Tb}^{3+}\right]_{1 / 2}$ of $301 \mu \mathrm{M}$ and max intensity 19 a.u. Although the inactive mutant had shown lower $\left[\mathrm{Tb}^{3+}\right]_{1 / 2}$, one should consider that the maximum intensity of the same sample is reduced as well. In summary, $\mathrm{Tb}^{3+}$ luminescence data confirmed that the inactive G6C mutant provides less binding sites in comparison to the active trimmed 6BX22.

A

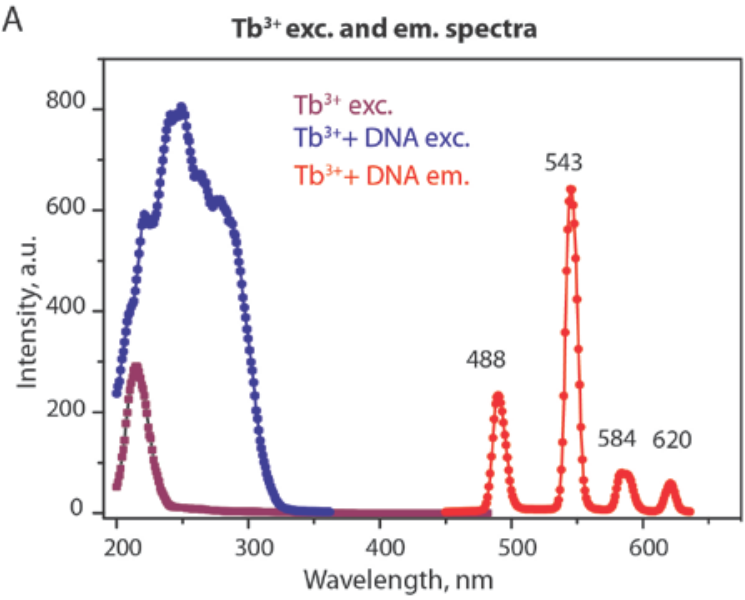

C

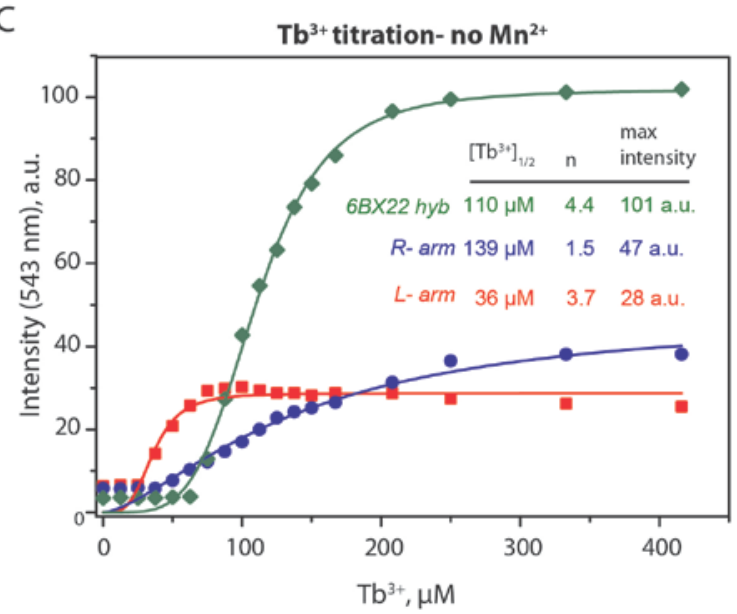

B

complexes used for this study
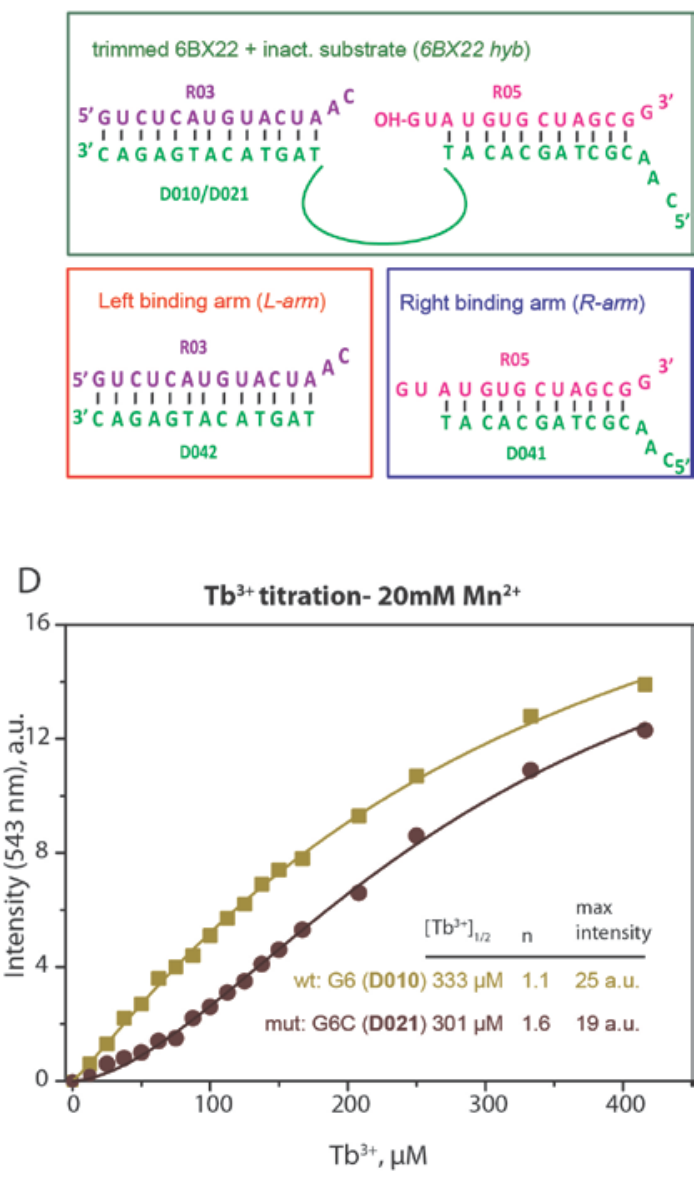

Fig 2.3-12 $\mathrm{Tb}^{3+}$ luminescence with trimmed 6BX22. A) Typical $\mathrm{Tb}^{3+}$ luminescence excitation and emission spectra. Excitation spectra are shown in the absence and presence of DNA. Emission spectrum is shown only in presence of DNA ( $2 \mu \mathrm{M}$ D010). B) shows the three DNA-RNA complexes that have been used in $\mathrm{C}$. Investigation of $\mathrm{Tb}^{3+} \mathrm{binding}$ to the catalytic core of $6 B X 22$ hyb was aimed here. Double stranded left and right binding arms are control samples for $\mathrm{Tb}^{3+}$ binding to DNA out of the catalytic core. C) $\mathrm{Tb}^{3+}$ titration to $6 B X 22$ hyb with active enzyme (D010) and the two binding arms, in presence of $50 \mathrm{mM}$ HEPES $\mathrm{pH} 7.5,150 \mathrm{mM} \mathrm{NaCl}$ and $2 \mathrm{mM} \mathrm{KCl}$. D) $\mathrm{Tb}^{3+}$ titration to $6 \mathrm{BX} 22$ hyb complexes with active trimmed 6BX22 (D010) or G6C inactive trimmed 6BX22 (D021) in the presence of $20 \mathrm{mM} \mathrm{MnCl}_{2}$. In C and $\mathrm{D}$ data were fit to the equation: $I=I_{\max } \cdot\left[\mathrm{Tb}^{3+}\right]^{\mathrm{n}} /\left(\left[\mathrm{Tb}^{3+}\right]^{\mathrm{n}}+\left[\mathrm{Tb}^{3+}\right]_{1 / 2}{ }^{\mathrm{n}}\right)$

\subsubsection{Circular dichroism (CD)}


Circular dichroism (CD) spectroscopy is an ideal method for tracing conformational transitions of large macromolecules. For DNA molecules, the phosphate-sugar backbone and global helical arrangement define their CD spectra. Different types of DNA helices i.e. A, B or Z, parallel or antiparallel Gquadruplex DNAs and I-motifs have specific known signatures in their CD spectra ${ }^{153}$. B-form of DNA has the signature with four peaks at $211 \mathrm{~nm}$ (negative), $221 \mathrm{~nm}$ (positive), $245 \mathrm{~nm}$ (negative) and $275 \mathrm{~nm}$ (positive) ${ }^{154}$. The characteristic signatures of B DNA are broadened in RNA-DNA hybrid ${ }^{155}$. A parallel intermolecular G-quadruplex exhibits a positive ellipticity band at $\sim 260 \mathrm{~nm}$ and a smaller negative band at $\sim 240 \mathrm{~nm}^{156,157}$, whereas an antiparallel G-quadruplex has a typical signature with a positive band at $295 \mathrm{~nm}$ and a negative band at $260 \mathrm{~nm}^{152}$.

CD spectra of trimmed 6BX22 showed a positive band at $270 \mathrm{~nm}$ and a smaller negative band at 240 $\mathrm{nm}$. While the negative band remained unchanged, the positive band was enhanced and shifted to 265 $\mathrm{nm}$ upon hybridization of the 6BX22 binding arms to the (inactivated) substrates. Standard active acceptor RNA (R01) and 5'-OH inactive donor RNA (R05) were used for binding arm hybridization. No additional changes were observed when the branched product was hybridized to the binding arms. Addition of $\mathrm{Tb}^{3+}$ (up to $150 \mu \mathrm{M}$ ), slightly decreased the positive band but left the negative band almost unaffected (Fig 2.3-13). In summary, CD data did not support any proof for presence of an anti-parallel G-quadruplex but was in agreement with presence of the RNA-DNA hybrid (binding arms) and/or parallel G-quadruplex in the 6BX22 enzyme.

A
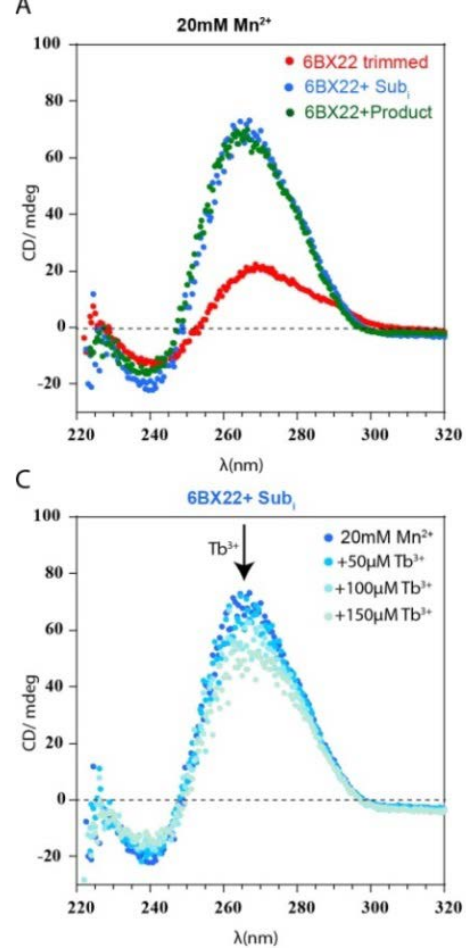

B

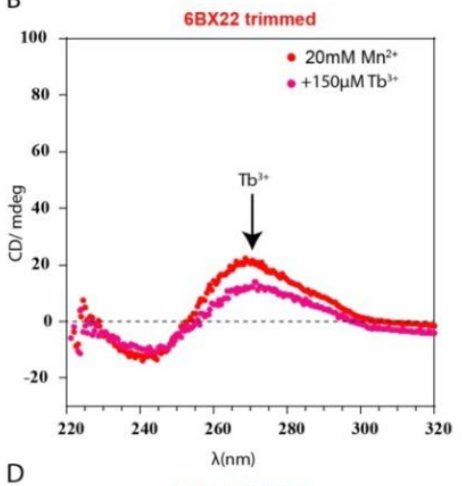

D

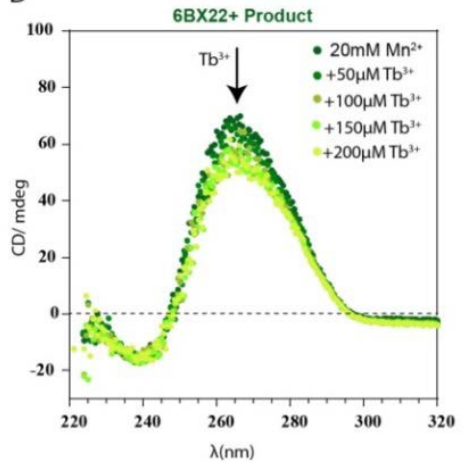

Fig 2.3-13 CD spectra of trimmed 6BX22 (3 $\mu M)$ in complex with inactivated substrates or branched product, in the presence of $50 \mathrm{mM}$ HEPES, $150 \mathrm{mM} \mathrm{NaCl}, 2 \mathrm{mM} \mathrm{KCl}, 20 \mathrm{mM}$ $\mathrm{MnCl}_{2}$ and increasing concentrations of $\mathrm{TbCl}_{3}$. 6BX22 shows a negative band at $240 \mathrm{~nm}$, which stays the same in presence of RNA substrates or branched RNA product. 6BX22 DNA also shows a positive band at $270 \mathrm{~nm}$ which shifts to $265 \mathrm{~nm}$ and is increased by hybridization of its arms to substrates or product (br2). Presence of increasing concentrations of $\mathrm{Tb}^{3+}$ ion reduced the intensity of the CD signal at $265 \mathbf{~ n m}$.

\subsubsection{Stabilization by TMPyP4 porphyrine}


Further investigation on presence of a G-quadruplex in 6BX22 was performed with biochemical and biophysical analyses of a G-quadruplex binding porphyrine. It had been shown that modified porphyrins with cationic side arms e.g. tetra-(N-methyl-4-pyridyl) porphyrin (TMPyP4) can stabilize Gquadruplexes ${ }^{158}$. TMPyP4 has been shown to stabilize telomeric G-quadruplexes and be an effective inhibitor of human telomerase. ${ }^{159}$ If a G-quadruplex is present in the $6 \mathrm{BX} 22$ catalytic core, it is conceivable that TMPyP4 mediated stabilization can decrease the dependence of the enzyme on metal ions. We have tested the activity of the enzyme in the presence or absence of $\mathrm{Mn}^{2+}$ and TMPyP4. (Fig 2.3-14) The dependence of the catalytic activity of the enzyme on $\mathrm{Mn}^{2+}$ was still remained and no acceleration of the enzyme was observed upon presence of 1- or 10-fold excess of TMPyP4.

Additionally, TMPyP4 is a fluorescent dye. Fluorescence spectra reflect information about the changes of the local environment of the fluorophors. It was reported that the shape and intensity of TMPyP4 emission spectra are altered upon interaction with G-quadruplexes. Excitation of intercalated TMPyP4 into DNA (at $433 \mathrm{~nm}$ ) results in an emission spectrum with two peaks at 660 and $720 \mathrm{~nm} .{ }^{160}$

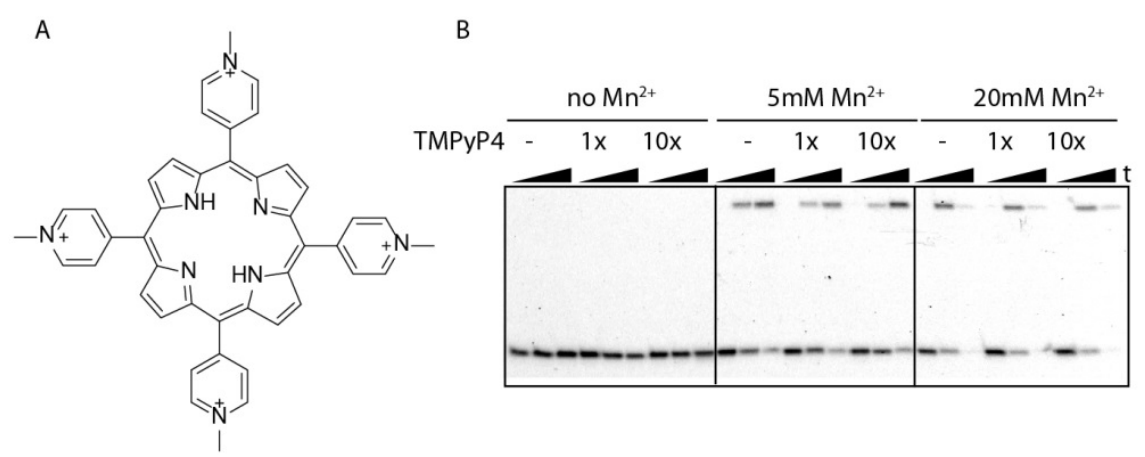

Fig 2.3-14 TMPyP4 structure and its effect on 6BX22 catalytic activity A) TMPyP4 structure. B) 6BX22 kinetics in presence or absence of TMPyP4 and $\mathrm{Mn}^{2+}$. Time points are 0,2 and $8 \mathrm{~h}$.
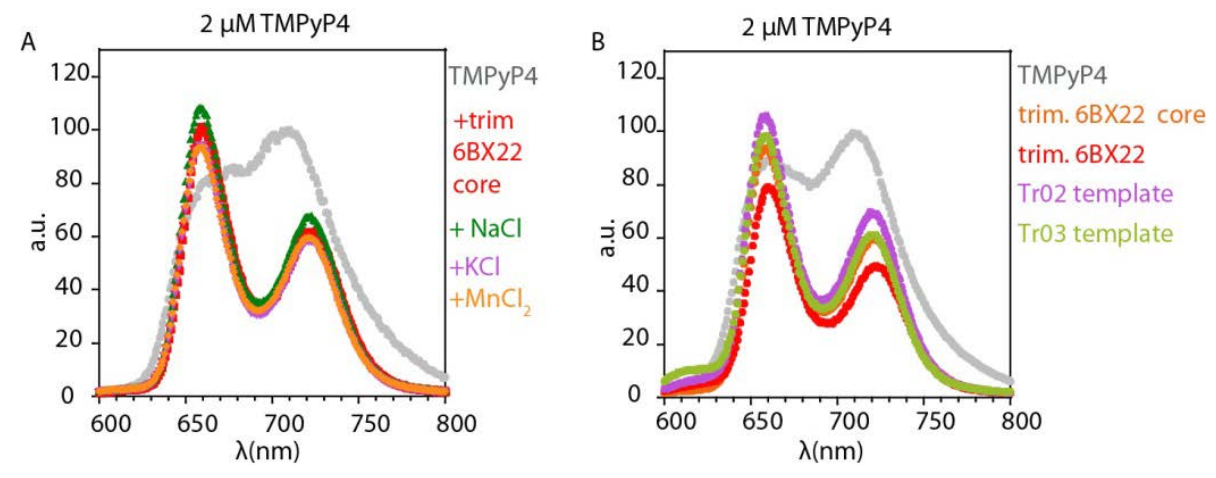

Fig 2.3-15 TMPyP4 fluorescence in presence of 6BX22 DNA and metal ions. A) Emission spectra of $2 \mu \mathrm{\mu M}$ TMPyP4 by stepwise addition of buffer (50 mM HEPES pH 7.5) plus DNA, minimized catalytic core (4 $\mu \mathrm{M}), \mathrm{NaCl}(150 \mathrm{mM}), \mathrm{KCl}(2$ $\mathrm{mM}$ ) and $\mathrm{MnCl}_{2}(20 \mathrm{mM})$. B) Emission spectra of $2 \mu \mathrm{MMpyP} 4$ in presence of $150 \mathrm{mM} \mathrm{NaCl}, 2 \mathrm{mM} \mathrm{KCl,} 20 \mathrm{mM}$ $\mathrm{MnCl}_{2}$ and $50 \mathrm{mM}$ HEPES $\mathrm{pH} 7.5$ and $4 \mu \mathrm{M}$ of 6BX22 related and unrelated DNAs.

Addition of metal ions (especially monovalents) to a DNA which forms a G-quadruplex increases the population of formed G-quadruplexes. To observe such effect, in the first experiments the emission spectra of $2 \mu \mathrm{M}$ TMPyP4 was monitored by stepwise addition of DNA, $\mathrm{NaCl}, \mathrm{KCl}$ and $\mathrm{MnCl}_{2}$. In this experiment, the trimmed 6BX22 catalytic core (without binding arms, D040) was used. Addition of 4 
$\mu \mathrm{M}$ DNA when $50 \mathrm{mM}$ HEPES pH 7.5 was present resulted in formation of the expected two peaks at 660 and $720 \mathrm{~nm}^{160}$ with ratio of 2:1 for the two peaks. Addition of metal ions did not lead to further significant changes in the emission spectra (Fig 2.3-15).

6BX22 minimized core with and without binding arms (D040 and D009) and also two DNAs unrelated to 6BX22 catalytic core i.e. DNA templates for transcription of Tr02 and Tr03 were added to TMPyP4. Formation of a G-quadruplex was not predictable according to the sequences of the two unrelated DNAs. Notably, the emission spectra of the 6BX22 related and unrelated DNAs were almost similar and even slightly higher intensities were observed for unrelated DNAs suggesting that the observed effect is nothing specific to the 6BX22 catalytic core and its hypothetical G-quadruplex (Fig 2.3-15 B). Increasing concentrations of DNAs up to $16 \mu \mathrm{M}$ (in presence of $2 \mu \mathrm{M}$ TMPyP4) did not cause additional significant increase in the fluorescence intensity for 6BX22 enzyme or unrelated DNAs (Fig 2.3-16 A). In a new experiment, TMPyP4 was titrated in presence of $20 \mu \mathrm{M} 6 \mathrm{BX} 22$ DNA or unrelated DNA. The observed intensity increased upon addition of the ligand. The fluorescence increase was more significant for the 6BX22 unrelated DNA (Fig 2.3-16 C).
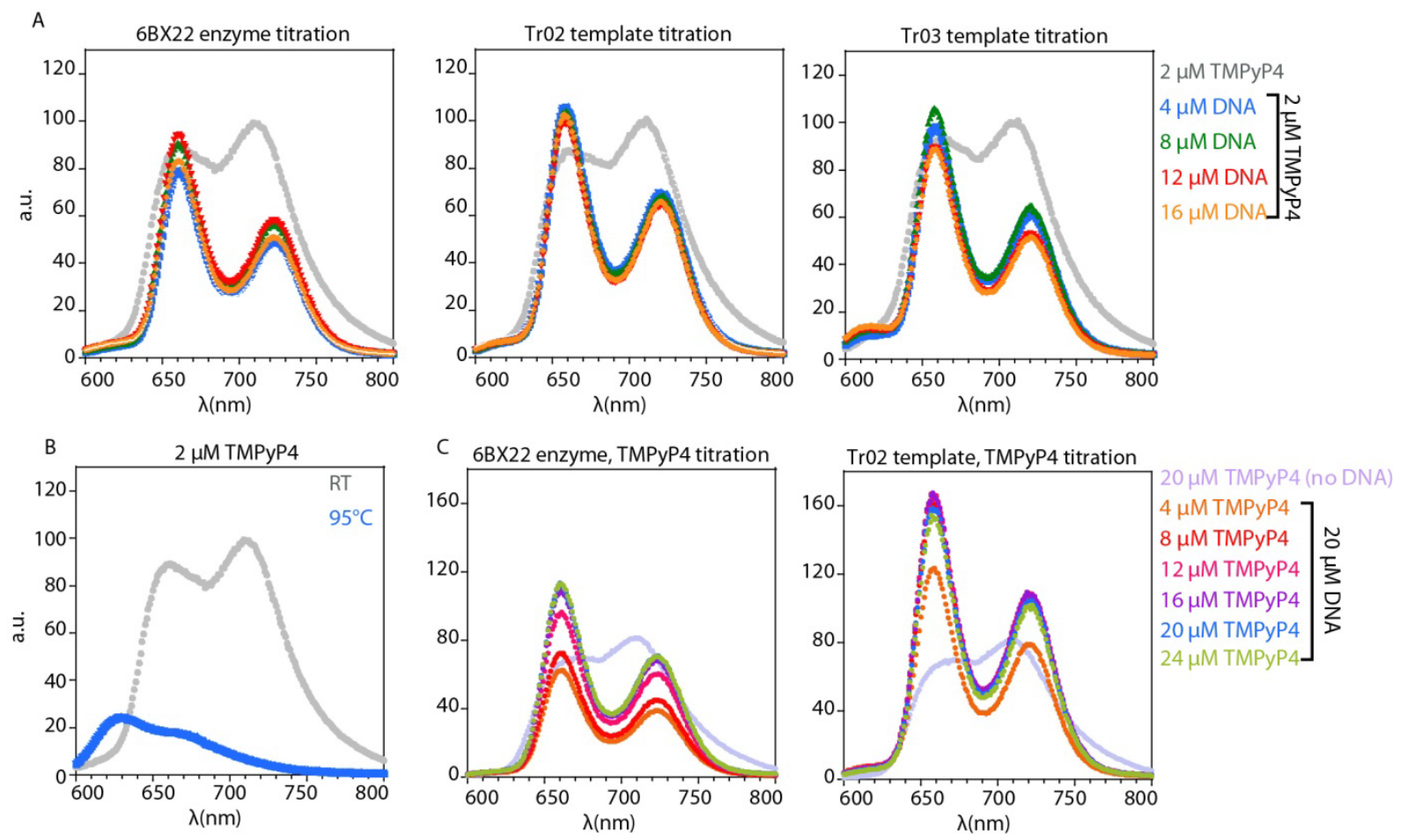

Fig 2.3-16 DNA and TMPyP4 titrations. A) emission spectra of $2 \mu \mathrm{M}$ TMPyP4 with increasing concentrations of 6BX22 enzyme (D009), or unrelated DNAs i.e. Tr02 template (D029) and Tr03 template (D030) in the presence of $50 \mathrm{mM}$ HEPES pH 7.5, $150 \mathrm{mM} \mathrm{NaCl}, 2 \mathrm{mM} \mathrm{KCl}$ and $20 \mathrm{mM} \mathrm{MnCl}_{2}$. B) TMPyP4 spectra before and after 1 min incubation at $95^{\circ} \mathrm{C}$. C) TMPyP4 titration in presence of $20 \mu \mathrm{M}$ DNA (D009 and D030). Increase in TMPyP4 concentration resulted in higher intensities. The effect is stronger for Tr02 DNA template. Additionally, emission spectrum of $20 \mu \mathrm{MM}$ TMPyP4 in the absence of DNA is plotted on the graphs in $\mathrm{C}$.

TMPyP4 was reported to stabilize G-quadruplexes. Stability of DNA structures are usually investigated by $\mathrm{UV}^{260 \mathrm{~nm}}$ or $\mathrm{CD}$ thermal melting analysis. Determination of melting temperature $\left(\mathrm{T}_{\mathrm{m}}\right)$ for $6 \mathrm{BX} 22$ in 
presence of TMPyP4 was not possible because the TMPyP4 was not stable at high temperature. Fig 2.3$16 \mathrm{~B}$ shows the emission spectra of TMPyP4 before and after 1 min incubation at $95^{\circ} \mathrm{C}$. In summary, biochemical and luminescence studies with TMPyP4 did not provide any proof in favor of presence of a G-quadruplex in 6BX22 catalytic core.

\subsubsection{BX22 foot printing assays}

Chemical DMS probing and enzymatic DNase I digestion have been used here to study 6BX22 folding. Footprinting assays such as dimethyl sulfate (DMS) probing or DNase probing reveal information on the accessibility of the $\mathrm{N}^{7}$ position of DNA guanines for methylation by DMS or phosphate-sugar backbone digestion by DNase. Conceivably, deoxyribozymes undergo structural changes upon hybridization to substrates, in presence of metal ions and in the course of reaction.

Here, three sample compositions have been used for foot printing assays; $\mathrm{D}^{\mathrm{t}}$, iS and brP. $\mathrm{D}^{\mathrm{t}}$ contained trimmed version of 6BX22 as sole nucleic acid component and resembled the pre-hybridization state. Complex iS had three components i.e. trimmed 6BX22, inactive acceptor substrate (R02) and inactive donor substrate (R05). Complex iS was representative of the pre-catalytic state. Complex brP contained trimmed 6BX22 hybridized to 6BX22 branched product and represented post-catalytic state of the enzyme.

For most of the positions in the catalytic core of the enzyme, DNase I accessibility of the backbone was diminished upon hybridization to the substrate ( $D^{t}$ vs iS). The protection was even further increased upon presence of branched product (brP). Examples for the described stepwise protection include $\mathrm{T} 11^{\mathrm{t}}$, $\mathrm{T} 12^{\mathrm{t}}, \mathrm{G} 13^{\mathrm{t}}, \mathrm{G} 16^{\mathrm{t}}, \mathrm{G} 24^{\mathrm{t}}, \mathrm{C} 26^{\mathrm{t}}$, and C27 ${ }^{\mathrm{t}}$. There are individual cases in which the protection increased in iS but reduced in brP complex. Examples for such positions include $\mathrm{C} 14^{\mathrm{t}}, \mathrm{A} 22^{\mathrm{t}}$ and $\mathrm{G} 23^{\mathrm{t}}$ (Fig 2.3-17 B).

Addition of buffer to the samples further decreased accessibility of DNA backbone for DNase I digestion. The protection level increased to the extent that almost no cleavage was observed for any of the complexes in presence of $150 \mathrm{mM} \mathrm{NaCl}, 2 \mathrm{mM} \mathrm{KCl}$ and $20 \mathrm{mM} \mathrm{MnCl}_{2}$. This is noteworthy since $\mathrm{MnCl}_{2}$ is an activator of DNase $\mathrm{I}^{145}$. For the DNase I digestion shown in Fig 2.3-17 B, $\mathrm{MnCl}_{2}$ was excluded from the buffer. In presence of buffer ( $\mathrm{Mn}^{2+}$ excluded) and in post catalytic state only $\mathrm{C}_{14}{ }^{\mathrm{t}}$ and $\mathrm{G}^{\mathrm{t}}{ }^{\mathrm{t}}$ were accessible for DNase I digestion. Comparing individual complexes of $\mathrm{D}^{\mathrm{t}}$, iS and brP in presence and absence of buffer shows that the buffer dependent protection occurs for each composition and is not dependent on the hybridization state and catalytic state of the DNA. For example $\mathrm{C} 27^{\mathrm{t}}$ was strongly cleaved in the absence of buffer but was protected in presence of buffer in all three sample compositions.

Global folding of the inactive DNA mutant G6A showed almost the same pattern as the active DNA with specific local differences (Fig 2.3-17 B, lanes 7-12). In the absence of buffer, the protection levels of $\mathrm{T} 11^{\mathrm{t}}, \mathrm{T} 12^{\mathrm{t}}$ and $\mathrm{G} 16^{\mathrm{t}}$ in brP complex with G6A were weaker compared to the active fold. The same 
positions and in the presence of buffer showed less protection in iS complex with inactive mutant G6A in comparison to the iS of the active enzyme. Additionally, The observed effect at C14 ${ }^{t}$ in active DNA (protection at iS but cleavage at brP) was not evident in the mutant.

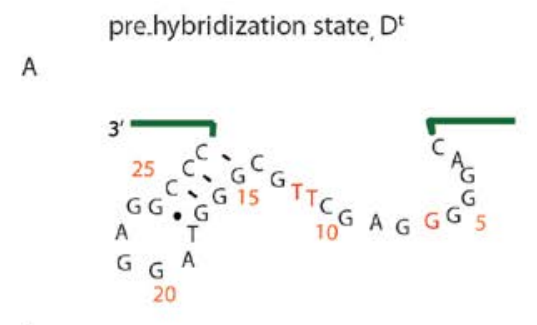

B

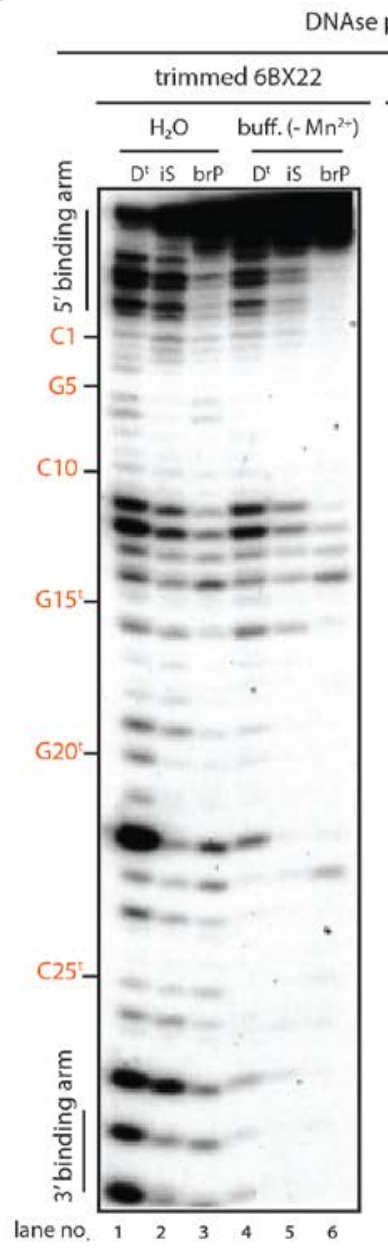

Pre.catalytic state, is

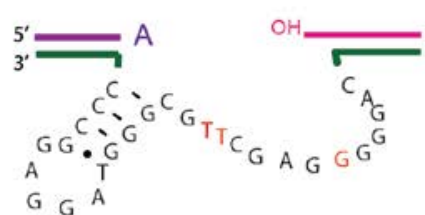

$G G^{A}$
Post.catalytic state brP

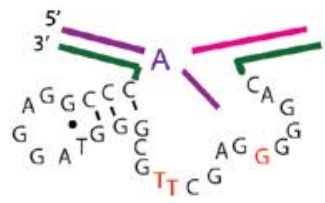

DMSprobing

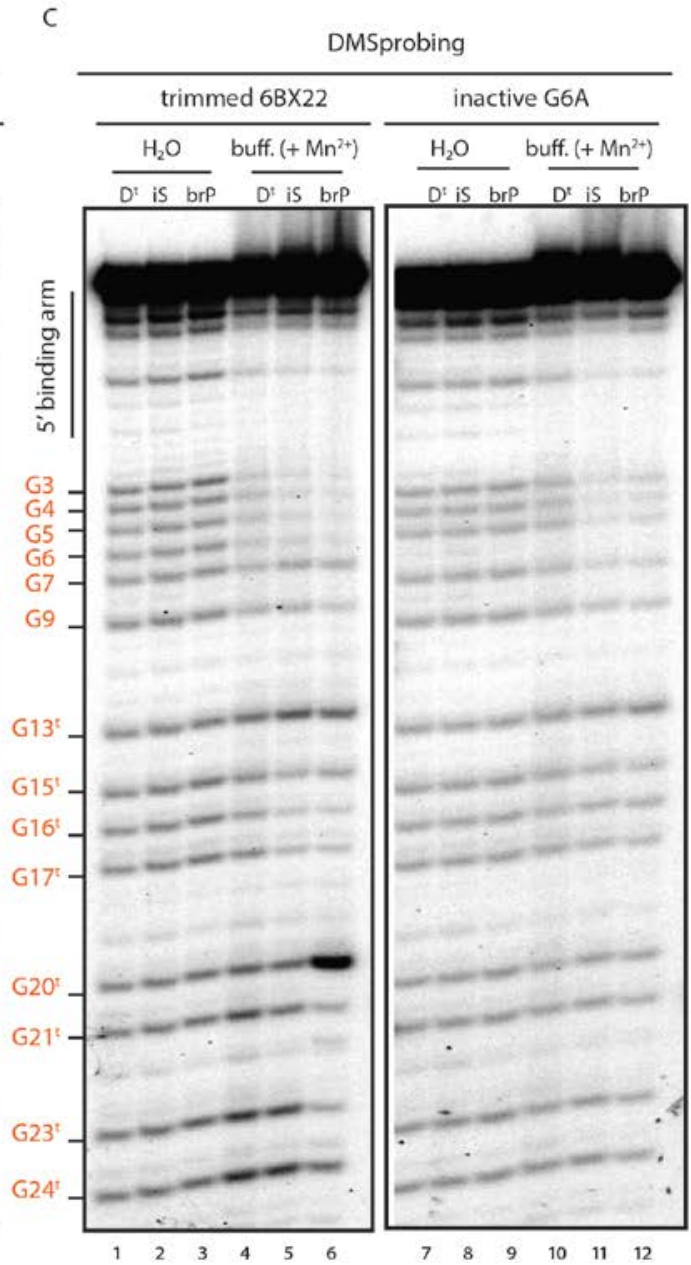

Fig 2.3-17 DMS and DNase I probing of minimized 6BX22 (D010) and its inactive point mutant; G6A (D015). DNAs were radiolabeled at 3' end. A) The three complexes used for probing assays. $D^{t}$ : DNA as sole component, iS: DNA in complex with inactive acceptor RNA (R002) and inactive donor RNA (R005), brP: DNA with branched product. The G6 position was mutated to A for samples marked with inactive DNA. B) DNase I probing. The buffer contains $50 \mathrm{mM}$ HEPES pH 7.5, $150 \mathrm{mM} \mathrm{NaCl,} 2 \mathrm{mM} \mathrm{KCl}$. $\mathrm{MnCl}_{2}$ is not present for DNase I probing. C) DMS probing, sample composition, buffer and metal ions are as in $\mathrm{B}$ except that $20 \mathrm{mM} \mathrm{MnCl}$ is additionally present in buffer lanes. (The stem loop is predicted by $\mathrm{m}-\mathrm{fold})$.

While the DNase I digestion reflects accessibility of the DNA backbone, DMS probing assay determines accessibility of the $\mathrm{N}^{7}$ positions of the guanines. For 6BX22 catalytic core and in the absence of buffer and $\mathrm{Mn}^{2+}$, there was no difference in methylation pattern of the enzyme comparing pre-hybridization and post-hybridization states. Presence of buffer and $\mathrm{Mn}^{2+}$ resulted in strong 
protection at positions G3-G6 in all three complexes, $\mathrm{D}^{\mathrm{t}}$, iS and brP. The protection level was increased from pre-hybridization state to pre- and post-catalytic states for these positions. G16, G17 ${ }^{\mathrm{t}}$ were only protected in post hybridization states (iS and brP). G21 ${ }^{t}$ and $G 23^{t}$ were only protected in post catalytic state (brP). G13 ${ }^{\mathrm{t}}$ remained unprotected in all cases (Fig 2.3-17 C, lanes 1-6).

Interestingly, accessibility of $\mathrm{G} 20^{t}$ was increased in post catalytic state when metal ions were present, despite the overall trend of increasing protection upon presence of metal ions or hybridization to the binding arms. Increased cleavage at G20 ${ }^{t}$ was not observed in the G6A inactive mutant (Fig 2.3-17 C, lane 6 and 12). We hypothesized that the product formation causes structural changes in the 6BX22 in which the position $\mathrm{G}^{\mathrm{t}} \mathrm{t}^{\mathrm{t}}$ protrudes from the core and becomes accessible for methylation by DMS.

To confirm this hypothesis, 6BX22 was annealed with its active substrates, R001 and Tr03 and incubated in the presence of $20 \mathrm{mM} \mathrm{Mn}^{2+}$. After indicated incubation times, samples were subjected to 10 min DMS methylation and further piperidine cleavage. The result showed that G3-G6 and G15 - G17 became protected upon presence of $\mathrm{Mn}^{2+}$. The protection maintained in the course of the reaction. Position G20 became more accessible for methylation over time as it was expected (Fig 2.3-18 A).

Kinetic data suggested that $\mathrm{Mn}^{2+}$ and $\mathrm{Tb}^{3+}$ compete for the same binding site in the 6BX22 catalytic core. Here, we investigated the effect of $\mathrm{Tb}^{3+}$ on the 6BX22 conformations which are formed in the presence or absence of $\mathrm{Mn}^{2+}$. In the presence of $20 \mathrm{mM} \mathrm{Mn}^{2+}$, increasing concentrations of $\mathrm{Tb}^{3+}$ up to $150 \mu \mathrm{M}$ did not perturb DMS pattern for pre-catalytic state. For post-catalytic state, the increased cleavage at $\mathrm{G} 20^{t}$ was unaffected but protection at G3-G6 was reduced by increasing concentrations of $\mathrm{Tb}^{3+}$ (Fig 2.3-18 B). DMS foot printing in presence of $150 \mu \mathrm{M} \mathrm{Tb}^{3+}$ and increasing concentrations of $\mathrm{Mn}^{2+}$ revealed the same methylation pattern in pre catalytic states but different pattern in post catalytic states (Fig 2.3-18 C). These data shows that, to form the specific signature of active enzyme with brP (G3-G6 protection and G20 protrusion) $\mathrm{Mn}^{2+}$ was required.

The inactivated complex used in DMS and DNase I (iS) consisted of inactive acceptor as well as inactive donor. Fig 2.3-18 D shows the DMS pattern of inactivated complex when the active donor is used (5'-ppp donor, Tr03). The only significant difference is at position $\mathrm{G} 17^{t}$ that is less protected in the presence of active donor. Altogether, foot printing assays revealed snapshots of structural changes of $6 \mathrm{BX} 22$ upon binding to its substrates, presence of metal ions especially $\mathrm{Mn}^{2+}$ and in the course of reaction. 


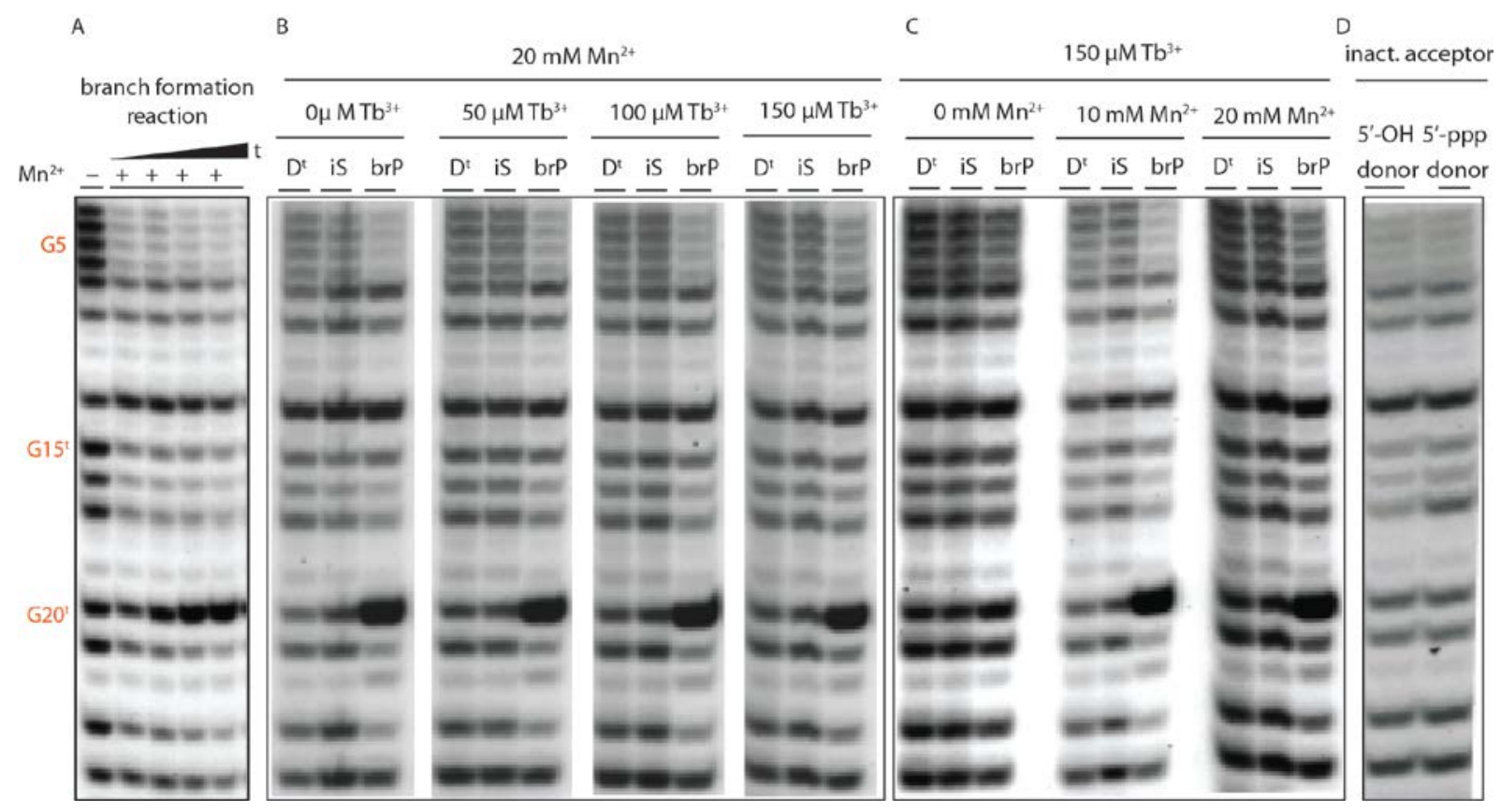

Fig 2.3-18 DMS probing of active 6BX22 and $\mathrm{Tb}^{3+}$ and $\mathrm{Mn}^{2+}$ titrations. A) DMS mediated cleavage pattern of active 6BX22 complex over time. All lanes contain $50 \mathrm{mM}$ HEPES pH 7.5, $150 \mathrm{NaCl}$ and $2 \mathrm{mM} \mathrm{KCl}$. 6BX22 reactions have been performed in presence of $20 \mathrm{mM} \mathrm{MnCl}_{2}$. Time points are $0,5,20$ and $90 \mathrm{~min}$. samples were further incubated at room temperature for 10 min for DMS methylation. B) DMS pattern of the three samples $\mathbf{D}^{t}$, iS and brP in presence of $20 \mathrm{mM} \mathrm{Mn}^{2+}$ and increasing concentration of $\mathrm{Tb}^{3+}$. C) DMS pattern of the three samples $\mathrm{D}^{\mathrm{t}}$, iS and brP in presence of $150 \mu \mathrm{M} \mathrm{Tb}^{3+}$ and increasing concentration of $\mathrm{Mn}^{2+}$. D) DMS probing with two different inactivated complex. The complex in both lanes are like the is complex, except that for 5'ppp lane the active donor RNA (Tr03) is used.

To summarize, footprinting assays on the trimmed 6BX22 revealed structural changes upon addition of buffer and metal ions. The cleavage pattern was also dependent on the hybridization status and the catalytic states. Cleavage at position G20 ${ }^{t}$ was strongly increased in the course of reaction.

\subsubsection{Discussion and outlook}

Our investigations about substrate requirements of 6BX22 revealed that regarding the acceptor RNA substrate, the first nucleotide adjacent to the 3' of the branch site adenosine is important for catalysis. In all substrates studied in this project, this position was occupied by a cytosine. One can hypothesize that this cytosine is employed for proper positioning of the branch site via Watson-Crick or Hoogsteen base pairing. In this case the identity of this cytosine must be important. Kinetic studies with point mutants of this cytosine would be useful to test this hypothesis.

Regarding the nucleotide requirement of the 6BX22 catalytic core, CoMA revealed that the region G11A24 and also the two nucleotides C38 and C39 accept all mutations. For all the other positions of 6BX22 either 2'-OH chemical tag or transition and/or transversion of the nucleobases were shown to be detrimental. We hypothesized that the mutable region G11-A24 serves only as a linker between the two sub domains of the catalytic core. The hypothesis was confirmed by kinetic studies on trimmed versions of 6BX22. In fact, the two parts of the original enzyme i.e. C1-C10 and G25-C39 required to be connected by a linker of at least 2 nucleotides long. The identity of the linker sequence was shown to be non-crucial. The linker was replaced by two thymidines in the trimmed 6BX22. Trimmed 6BX22 was a 
slightly better catalyst compared to the original 6BX22 and maintained the ability of forming lariat RNAs. From this point of discussion, for consistency, the numbering of the nucleobases are based on the trimmed 6BX22 and are marked with superscript letter "t”, except otherwise mentioned.

The trimmed 6BX22 was 27 nucleotides long and contained 14 guanosines. High G-content and the fact that the guanosines' arrangement in the catalytic core accommodated four times two guanosines in a row raised the idea of presence of a G-quadruplex in the catalytic core of 6BX22. G-quadruplexes adopt different topologies and can be formed with parallel or antiparallel DNA strands. The participating strands can be from different molecules (inter-molecular G-quadruplexes) or within the same DNA molecule, forming several loop structures to bring the four strands in position (intra-molecular Gquadruplexes) ${ }^{161}$. G-quadruplexes are stabilized normally by a monovalent metal ion in the core of the G-tetrad. Bivalent metal ions e.g. $\mathrm{Sr}^{2+}$ and $\mathrm{Pb}^{2+}$ also have been shown to effectively stabilize such structures in vitro ${ }^{162}$.

Porphyrine dyes with fluorescent properties have been suggested to selectively bind to G-quadruplexes and discriminate between G-quadruplexes and DNA double helices. ${ }^{163}$ Porphyrine dyes are also reported to stabilize such structures by axial stacking or intercalation to the G-quartet ${ }^{158}$. G-quadruplexes are also known for specific CD spectra for parallel and anti-parallel forms of them ${ }^{156,164}$. Presence of a Gquadruplex in 6BX22 catalytic core was investigated by several methods, including CD spectroscopy, kinetic and fluorescent studies using G-quadruplex stabilizing agent TMPyP4, dNAIM, DMSi, DMS and DNase I probing and studies on metal ion dependence of the enzyme.

Sugar conformation of guanines of G-quadruplexes has been studied using spectroscopic methods such as Raman spectroscopy ${ }^{165}$. Studies by Raman spectroscopy revealed that in a parallel G-quadruplex the sugar base conformation are C2'-endo/anti dG as in B DNA. In an antiparallel G-quadruplex, it was suggested that both C2'-endo/syn dG besides C2'-endo/anti dG are present ${ }^{166,167}$.

Presence or absence of 2'-OH of the ribose, may alter the sugar-base conformation. In a B DNAs sugars adapt the conformation of C-2'-endo. Sugar pucker of the RNA monomers are also C-2'-endo ${ }^{168}$ but in an A form RNA (and DNA) C-3'-endo is favored due to steric problems. The deoxyriboses bearing C$3^{\prime}$ '-endo tilt $19^{\circ}$ from the helix axis, in comparison to the riboses with C-2'-endo conformation ${ }^{169}$. CoMA employs the 2'-OH as a chemical tag which can affect the sugar conformation of deoxyribozymes' nucleosides.

In CoMA analysis, sensitive positions to the 2'-OH tag were G3, G5, G6, C14 ${ }^{\mathrm{t}}, \mathrm{G} 16^{\mathrm{t}}$ and $\mathrm{G} 20^{\mathrm{t}}$. In case that a G-quadruplex is present in the 6BX22 structure, one can deduce that the sugar pucker of the participating guanosines prefers to be $\mathrm{C}-2$ '-endo. Presence of 2'-OH tag can switch the conformation to C-3'-endo and therefore perturb the formation of the G-quadruplex. Here, the interference of the 2'-OH at these positions cannot directly confirm involvement of these positions in formation of a G-quadruplex 
but it is in agreement with this hypothesis. The 2'-OH sensitivity can be associated to the dependence of the catalytically active fold of 6BX22 to conformations of those sugar at those positions.

dNAIM of trimmed 6BX22 revealed interesting data about the involvement of individual functional groups of 6BX22 necessary guanines. Four modifications have been studied here. $c^{7} G$ dNAIM and DMSi $\left(\mathrm{m}^{7} \mathrm{G}\right)$ provided information about the Hoogsteen side of the guanines. $\mathrm{m}^{1} \mathrm{G}$ and $2 \mathrm{AP}$ dNAIMs provided information about the Watson-Crick side of the guanines.

$c^{7} G$ dNAIM and DMSi data both revealed that positions G7, G13 ${ }^{t}$ and $G 15^{t}$ of the catalytic core of the enzyme do not require the $\mathrm{N}^{7}$ position of the purine ring and can accept presence of a bulky methyl group at this position (interference values below 2 for both $c^{7} G$ dNAIM and DMSi). G4, G9, G17 $\mathrm{G} 20^{\mathrm{t}}, \mathrm{G} 23^{\mathrm{t}}$ and $\mathrm{G} 24^{\mathrm{t}}$ had interference values between $2-5$ for at least one of the two libraries $\mathrm{c}^{7} \mathrm{G}$ and $\mathrm{m}^{7} \mathrm{G}$. The interference values between 2-5 reflect that the enzymatic activity of the enzyme was reduced but was not abolished.

Regarding the Watson-Crick side, for $\mathrm{G}^{\mathrm{t}}{ }^{\mathrm{t}}$, the interference values of both $2 \mathrm{AP}$ and $\mathrm{m}^{1} \mathrm{G}$ were slightly above threshold (between 2-5). Our dNAIM libraries did not include any modification regarding position 2 of the guanine ring and its primary amino group. The fact that CoMA showed $\mathrm{G}^{\mathrm{t}}{ }^{\mathrm{t}}$ is not mutable to any other nucleotide and at the same time $2 \mathrm{AP}, \mathrm{c}^{7} \mathrm{G}$ and $\mathrm{m}^{7} \mathrm{G}$ were tolerated allows deducing that $G 13^{t}$ is involved in catalysis by a non-canonical base pairing using the nitrogen at position 1 or amino group at position 2 .

Regarding the Watson-Crick side of the G7, presence of a methyl group at position 1 of the guanine ring $\left(\mathrm{m}^{1} \mathrm{G}\right)$ was not tolerated but the keto group at position 6 of the guanine ring could be removed (2AP) with no perturbation for catalysis. According to CoMA for G7 only A mutation was tolerated. Mutation tolerance from $\mathrm{G}$ to $\mathrm{A}$ excluded requirement of the Watson-Crick side functional groups. $\mathrm{N}^{7}$ modification tolerance at G7 excluded requirements of the Hoogsteen side of this position. The only detrimental modification was $\mathrm{m}^{1} \mathrm{G}$. Taken together one can conclude that position 7 must be a purine to maintain positioning and distances of the other nucleotides. It can be hypothesized that this position only plays a steric role for formation of the active structure. It is conceivable that G7 is buried inside the 6BX22 structure and therefore presence of a bulky methyl group at position 1 of the guanine ring is not tolerated. A similar role as G7 is predictable for G15. G15 ${ }^{\mathrm{t}}$ accepts all the tested modifications and mutation to A but not to $\mathrm{C}$ and $\mathrm{U}$.

The only positions with disruptive modifications for both $c^{7} G$ and $m^{7} G$ libraries were G3, G5, G6, G16 ${ }^{t}$ and $\mathrm{G} 21^{\mathrm{t}}$. At these positions any type of modification at $\mathrm{N}^{7}$ is detrimental and the enzymatic activity of the enzyme is strongly dependent on the Hoogsteen side. From these five positions $\mathrm{G}_{2} 1^{\mathrm{t}}$ requires both Hoogsteen and Watson-Crick sides. G5 accepts $\mathrm{m}^{1} \mathrm{G}$ modification in Watson-Crick side. G3, G6 and G16 ${ }^{t}$ have been shown to be sensitive to 2'-OH, therefore we don't have any data about their WatsonCrick sides. Altogether the only guanines for which requirement of both sides are not disproved are G3, 
G6, G16 ${ }^{t}$ and G21 ${ }^{t}$. These four positions are not sufficient to form a standard G-quadruplex in $6 \mathrm{BX} 22$ catalytic core. The three dimensional structure of the 6BX22 is therefore not a simple known DNA motif but rather a complicated structures including non-canonical base pairings. In the next paragraphs interesting individual cases are discusses separately to pinpoint constraints about 6BX22 threedimensional structure.

$c^{7} G$ modified G does not provide the nitrogen atom at position 7 of the modified guanine for formation of hypothetical hydrogen bond or metal ion coordination. In the same track but with slight differences, DMS mediated methylation perturbs the hydrogen bonds or metal ion coordination of $\mathrm{N}^{7}$ by both steric hindrance and electronic changes. $c^{7}$ G dNAIM and DMSi of 6BX22 showed similar interference or tolerance for all positions except at four positions. G17 ${ }^{\mathrm{t}}$ and $\mathrm{G} 23^{\mathrm{t}}$ tolerated the absence of $\mathrm{N}^{7}$ but did not tolerate $\mathrm{N}^{7}$ methylation. In the other hand, despite the tolerance to $\mathrm{N}^{7}$ methylation at G4 and $\mathrm{G} 20^{\mathrm{t}}$, the interference values of these positions for $\mathrm{c}^{7} \mathrm{G}$ modifications were significant.
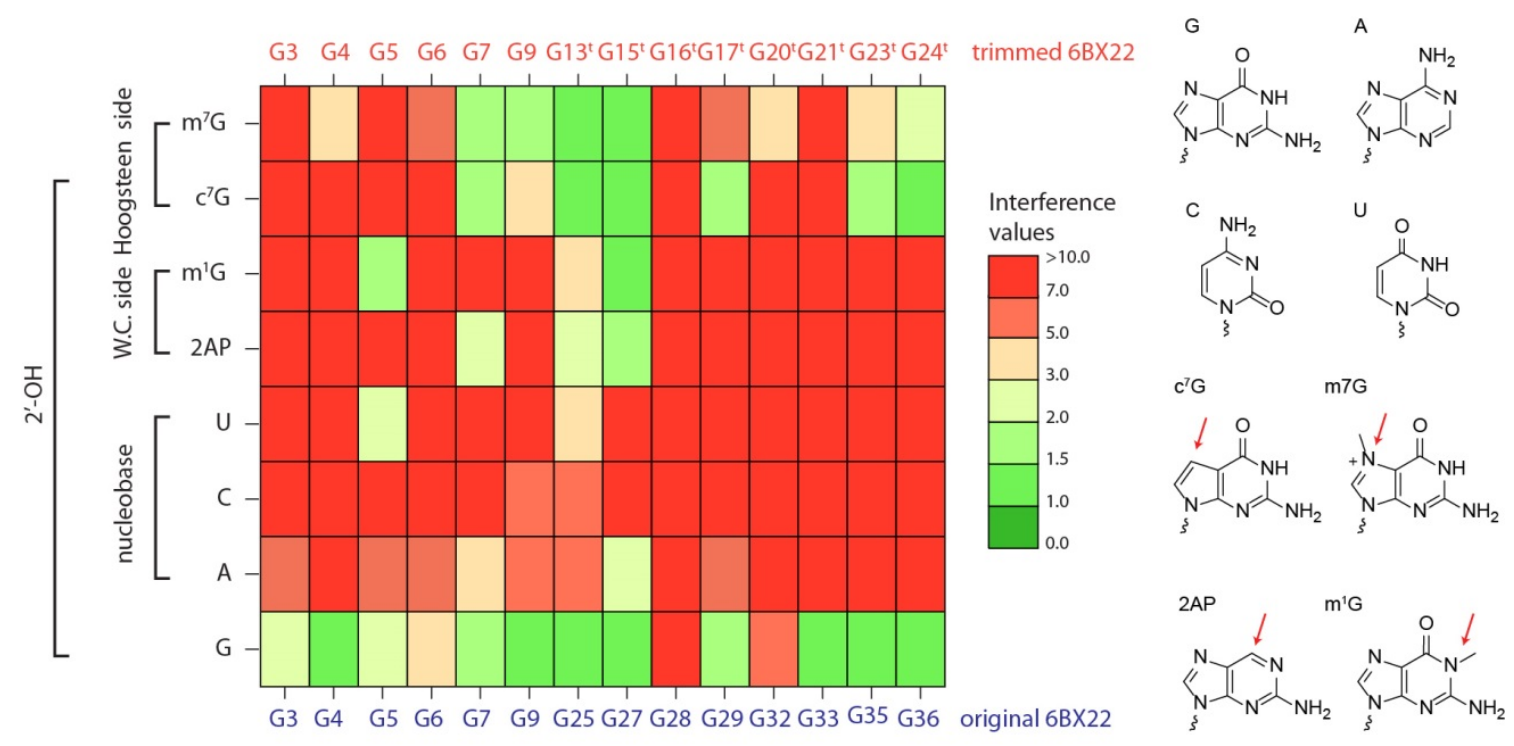

Fig 2.3-19 Comparison of CoMA and dNAIM data for necessary guanines of 6BX22 catalytic core. Numberings based on original and trimmed 6BX22 core are marked with navy and orange. CoMA was performed on original 6BX22 while dNAIM was performed on trimmed 6BX22.

For G20 ${ }^{t}$, the 2'-OH was shown to be detrimental in rG library therefore the $c^{7} G$ interference value does not necessarily reflect the modification effect. In the case of $G 17^{t}$ and $G 23^{t}$ it is conceivable that $\mathrm{N}^{7}$ is not directly involved in formation of a hydrogen bond or metal ion coordination, but methylation at this site disturbs the structure by steric hindrance.

dNAIM showed that methylation of G5 at position 1 of the nucleobase $\left(\mathrm{m}^{1} \mathrm{G}\right)$ is tolerated which excludes the possibility of formation of canonical Watson-Crick base pairing with G5. Despite $\mathrm{m}^{1} \mathrm{G}$ tolerance, removal of the keto group from the position 6 of the guanine nucleobases (2AP) was shown to be detrimental at this position. Taking CoMA data into account, G5C and G5A were detrimental while 
G5U was tolerated. Uridine has a keto group at position 4 of its pyrimidine ring. Together, data about G5 suggested involvement of G5 keto group in formation of the catalytic core.

Furthermore, considering the Hoogsteen side of the G5, $c^{7} \mathrm{G}$ dNAIM and DMSi suggested involvement of $\mathrm{N}^{7}$ position of G5 in catalysis. The two methods showed that position 7 of the G5 needs to be occupied by a nitrogen atom, and the methylation of this nitrogen is not tolerated. Considering that the enzyme tolerates G5U mutation and uracil lacks the imidazole ring, raises the question that in the case of G5U mutation what functional group compensates the absence of $\mathrm{N}^{7}$ of G5. Further investigations are required to answer this question.

Performing CoMA with a new set of libraries in which the position 5 of the 6BX22 catalytic core is mutated to uridine might be helpful to find the nucleobase(s) which compensates the lack of $\mathrm{N}^{7}$ at this position. For solid phase synthesis of the new libraries, mixtures of $\mathrm{rN} / \mathrm{dN}$ should be used for synthesis of all of the positions of the catalytic core except for the position 5 in which deoxyribonucleotide uridine phopshoramidite is suggested to be used. Nucleotide analogue interference suppression (NAIS $)^{170}$ has conceptual similarities to the suggested method, with the difference that in NAIS a detrimental modification is retrieved upon additional modification compensating the role of the first modification. In contrast, here the mutation G5U is active and comparing the CoMA pattern of wild type G5 6BX22 and mutant G5U will provide information about nucleobases compensating the absence of the keto group.

Disregard of the hypothesis of presence of a G-quadruplex, the secondary structure of the enzyme was reported to have a stem loop structure, predicted by m-fold. The stem was predicted to be formed by G15 $-T 18^{t}$ and G24t-C27 (G27-T30 and G36-C39 of original 6BX22 sequence). CoMA confirmed 6BX22 catalysis is independent of $\mathrm{C}^{\mathrm{t}} \mathrm{t}^{\mathrm{t}}$ and $\mathrm{C} 27^{\mathrm{t}}$ (C38-C39 in original sequence). Additionally, G15 ${ }^{\mathrm{t}}$ (G27 originally) tolerated modifications at its Watson-Crick side ( $\mathrm{m}^{1} \mathrm{G}$ and 2AP libraries). These data disproved possibility of formation of such stem loop structure in 6BX22 catalytic core.

Although, dNAIM rejected presence of a G-quadruplex in the catalytic core of the enzyme, additional biochemical and biophysical studies have been performed to further investigate presence or absence of the G-quadruplex. Most of the data either disproved or did not support presence of a G-quadruplex in this enzyme.

G-quadruplexes are mainly dependent on monovalent metal ions ${ }^{124}$. Our data suggested no significant dependence of 6BX22 on monovalent metal ions, but on $\mathrm{Mn}^{2+}$. It is known that G-quadruplexes are destabilized upon presence of $\mathrm{Mn}^{2+}$ metal ions presumably due to the coordination to nucleophilic atoms (N-7 and O-6) of guanine bases ${ }^{156}$. Bivalent metal ions such as $\mathrm{Ca}^{2+}, \mathrm{Sr}^{2+}$ and $\mathrm{Pb}^{2+}$ are shown to stabilize G-quadruplexes ${ }^{171,172}$. It was shown that $6 \mathrm{BX} 22$ is not active in presence of $\mathrm{Ca}^{2+26}$ but the activity of the enzyme in presence of $\mathrm{Pb}^{2+}$ and $\mathrm{Sr}^{2+}$ was not tested. $\mathrm{Tb}^{3+}$ was shown to occupy the central cavity of the G-quadruplexes ${ }^{124}$. Here we studied the catalytic activity of the 6BX22 deoxyribozyme in presence of 
$\mathrm{Tb}^{3+}$. Our data showed reversible inhibition by $\mathrm{Tb}^{3+}$ and suggested that $\mathrm{Tb}^{3+}$ and $\mathrm{Mn}^{2+}$ compete for the same binding site which is catalytically important. Considering the same binding site for $\mathrm{Mn}^{2+}$ and $\mathrm{Tb}^{3+}$ excluded the possibility of binding of the inhibitory $\mathrm{Tb}^{3+}$ to the cavity of the hypothetical G-quartet because it is known that $\mathrm{Mn}^{2+}$ is not capable of occupying the metal ion site of a G-quartet. Mainly, our kinetic analysis did not support or disprove presence of a G-quadruplex in 6BX22.

Kinetic investigations of the enzyme in presence of a G-quadruplex stabilizer, TMPyP4 did not show any enhancement of the 6BX22 enzymatic activity. Also, the dependence of the enzyme to $\mathrm{Mn}^{2+}$ metal ion was maintained. TMPyP4 is a fluorescent dye which is shown to change its emission spectra upon binding to G-quadruplexes. Upon presence of DNA the fluorescence emission spectra of TMPyP4 changed to reach the two expected peaks but the effect was not exclusively for the 6BX22 and its hypothetical G-quadruplex. Two unrelated DNAs (regarding 6BX22 sequence), in which presence of a G-quadruplex was not predictable, were shown to have the same effect as 6BX22. It can be concluded that the TMPyP4 has changed its emission spectra only upon non-specific intercalation to DNAs. TMPyP4 is traditionally considered as an stabilizer of G-quadruplexes but more recently its binding to other DNA motifs such as three-helix junctions ${ }^{173}$, poly dG:dC and poly dT:dA DNA duplexes as well as polypyrenotides is also reported ${ }^{174}$.

The positive band at CD spectra of 6BX22 DNA (at $270 \mathrm{~nm}$ ) with a slight blue-shift upon hybridization to substrates (to $265 \mathrm{~nm}$ ) rejected the possibility of presence an anti-parallel G-quadruplex. The positive band of 6BX22 was in between the positive band of parallel G-quadruplex (260 nm) and B-form DNA (275 nm). The negative band of 6BX22 at $245 \mathrm{~nm}$ was also reported to be a signature for both B-DNA and parallel G-quadruplex CD spectra. Altogether, CD data did not directly point toward any of those structures.

DMS and DNase I foot printing assays have been used to monitor global and local folding of 6BX22 in pre-hybridization and post hybridization states (pre- and post-catalytic). The 6BX22 global fold became more compact in post hybridization states and also upon presence of metal ions. The two factors, hybridization and metal ions, altered the structure in parallel pathways and independent from each other, but had shown synergistic effects. The non-hybridized DNA in water was the least protected and the DNA hybridized to the branched product in presence of buffer was the most protected. G20 ${ }^{t}$ was an exception to this general observation (in DMS probing). All these data confirm that 6BX22 has a dynamic structure with three distinct conformations. The 6BX22 global fold becomes more compact upon hybridization and presence of metal ions to achieve the "open form" of the enzyme-substrate complex. Formation of the 2',5' branched product causes changes in the structure and leads to "closed form” of the enzyme-product complex.

In DMS probing, the post hybridization protection at G3-G6 and post catalytic protrusion of G20 ${ }^{t}$ was shown to be the signature of the "native" conformation. The word native is quoted since 
deoxyribozymes are only synthetic DNAs. The two signs were confirmed by DMS probing of the 6BX22 enzyme using active substrates and in presence of standard reaction condition in the course of reaction. While the G3-G6 became stably protected upon presence of metal ions, the G20 ${ }^{t}$ became more accessible in the course of reaction which can be associated to the formation of product.

Our kinetic data suggested that $\mathrm{Tb}^{3+}$ and $\mathrm{Mn}^{2+}$ compete for the same binding site in the catalytic core of the enzyme. More investigations with DMS showed that $\mathrm{Tb}^{3+}$ is not sufficient to form the active conformations of the enzyme in the absence of $\mathrm{Mn}^{2+}$. In cases that both $\mathrm{Mn}^{2+}$ and inhibitory concentrations of $\mathrm{Tb}^{3+}$ were present, the G20 ${ }^{t}$ protrusion was unaffected while G3-G6 protections were diminished. These observations could indicate that $\mathrm{Tb}^{3+}$ interferes with formation of the compact active site which is the source of $\mathrm{Tb}^{3+}$ inhibition for 6BX22. The DMS data did not provide additional support for the hypothesis of the same binding site for $\mathrm{Mn}^{2+}$ and $\mathrm{Tb}^{3+}$, it also did not disprove it. It is conceivable, that G3-G6 participate in formation of a metal ion binding site for which both $\mathrm{Mn}^{2+}$ and $\mathrm{Tb}^{3+}$ compete.

However both DNase and DMS data were in agreement of structural changes of the enzyme upon hybridization to its substrate or product, but there are differences in the cleavage patterns of the two assays which should be noticed. In DMS probing $\mathrm{G}^{\mathrm{t}}{ }^{\mathrm{t}}$ accessibility to methylation remains unaffected upon presence of metal ions or substrate/products while accessibility of the DNA backbone of the same position was reduced in the course of reaction. G20t backbone remained protected in both post hybridization states while the methylation based cleavage at the same position was enhanced in post catalytic state. The DNA backbone at position G23 was more prone to cleavage at post catalytic state while methylation of $\mathrm{N}^{7}$ position of the same guanosine was decreased. The conformational changes of the DNA backbone likely affect the syn-anti conformation of the nucleobase ${ }^{175}$ and thereby accessibility or inaccessibility of the $\mathrm{N}^{7}$ position.

CD data suggested a dramatic change in the chirality and global folding of the DNA (pre-hybridization state) upon hybridization to RNA substrates (pre-catalytic state), but no further change by hybridization to the branched product (post-catalytic state). Conversely, DMS and DNase I probing assays showed significant changes upon hybridization and also over the course of reaction between the two posthybridization states. The difference in the two sets of data might arise from the fact that the two methods monitor different aspects of the three dimensional fold.

Our kinetic data showed reversible inhibition of $\mathrm{Mn}^{2+}$ dependent 6BX22 reaction upon presence of micromolar concentrations of $\mathrm{Tb}^{3+}$. Spectroscopic properties of $\mathrm{Tb}^{3+}$ are enhanced upon binding to DNA, RNA or organic metal ion chelators like EDTA. Sensitized luminescence of $\mathrm{Tb}^{3+}$ has been used to study metal ion binding sites of ribozymes ${ }^{113}$. Here we investigated metal ion binding sites of 6BX22 in hybridized form to its substrates by $\mathrm{Tb}^{3+}$ luminescence. In the case of 6BX22, we were not interested in the hypothetical metal ion binding sites in the linker region (mutable region), therefore $\mathrm{Tb}^{3+}$ 
luminescence studies were performed employing trimmed 6BX22. $\mathrm{Tb}^{3+}$ luminescence data of trimmed $6 \mathrm{BX} 22$ stalled in pre-catalytic state, "6BX22 hyb", and the two controls for non-specific metal ion binding sites of double stranded binding arms, confirmed that although the luminescence data are not simply additive, but the luminescence intensity of the $6 B X 22$ hyb containing both binding arms and the catalytic core are much higher than each of individual binding arms, reflecting more $\mathrm{Tb}^{3+}$ binding sites in the $6 B X 22$ hyb complex. This set of experiments have been performed in the absence of $\mathrm{Mn}^{2+}$ to pin point all the possible metal ion binding sites of the 6BX22.

Foot printing assays have shown that $\mathrm{Mn}^{2+}$ is necessary to form the active structure of the enzyme. Therefore, in a new $\mathrm{Tb}^{3+}$ sensitized luminescence experiment, $\mathrm{Tb}^{3+}$ titration was performed in presence of $20 \mathrm{mM} \mathrm{Mn}^{2+}$ to compare the active enzyme and its inactive mutant, G6C. The inactive mutant showed lower $\left[\mathrm{Tb}^{3+}\right]_{1 / 2}$ and lower maximum intensity compared to the active enzyme. In this titration experiments the observed luminescence is associated to the replacement of $\mathrm{Mn}^{2+}$ by $\mathrm{Tb}^{3+}$ because the samples have been folded in presence of $\mathrm{Mn}^{2+}$.

Hypothesizing that G6 involves in formation of the binding site for the catalytically important $\mathrm{Mn}^{2+}$, and considering that $\mathrm{Tb}^{3+}$ and $\mathrm{Mn}^{2+}$ compete for the same binding site which is catalytically important, it could be deduced that disruption of the $\mathrm{Mn}^{2+}$ binding site (G6C) disrupts the $\mathrm{Tb}^{3+}$ binding site. Our data supported this hypothesis, G6C provided less metal ion binding sites for $\mathrm{Tb}^{3+}$ and had reduced maximum luminescence intensity. Notably, the $\left[\mathrm{Tb}^{3+}\right]_{1 / 2}$ of the mutant was lower than the active trimmed enzyme. It can be hypothesized that G6C mutation disrupts the catalytically relevant metal ion binding sites and the conformational effect of such mutation increases the affinity of other metal ion binding sites to $\mathrm{Tb}^{3+}$.

In summary, 6BX22 enzyme was trimmed to almost 70\% of its original length using CoMA data. More than $50 \%$ of the nucleobases of the trimmed 6BX22 were guanosines. Presence of a G-quadruplex was investigated by several methods. Kinetic studies in presence of different metal ions and TMPyP4 neither proved or disproved the hypothesis. Presence of a G-quadruplex was supported by CD data but was declined strongly by dNAIM data. DMS and DNase probing assays suggested dynamic conformational changes of the enzyme catalytic core upon hybridization to the RNA substrates, presence of metal ions and in the course of reaction.

Transition from "open” state to the "closed" state was shown to be accomplished by protrusion of the DNA backbone at G23 and the guanine nucleobases at G20. At the same time the guanine nucleobases of G23 becomes buried inside the structure. The DNA backbone and guanine nucleobases of G3-G6 remain protected upon presence of the metal ion in both open and closed states. It is likely that this region is involved in formation of the catalytic center of the enzyme. dNAIM and CoMA data revealed importance of the keto group of G5 and DMS probing and $\mathrm{Tb}^{3+}$ luminescence data revealed importance of G6 for formation of metal ion binding site for catalytically active $\mathrm{Mn}^{2+}$. 


\section{Application of ligating deoxyribozymes}

Functional characterization of deoxyribozymes led to the discovery of conditions which enhance catalysis of formation of 2',5'- branched RNA (Section 2.2) with three helix junction forming deoxyribozymes e.g. 10DM24 and 9F7 deoxyribozyme family. The reaction rates were shown to be accelerated up to 10000-fold and 2',5'-branched RNA formation became efficient at low concentrations of bivalent metal ions e.g. $7 \mathrm{mM} \mathrm{Mg}{ }^{2+}$. These findings encouraged us to expand application of 2',5'branched RNA forming deoxyribozymes.

Despite the fact that branched RNAs can be potentially used as a tool in cellular and molecular biology and nanotechnology, few reports are available on their application since the synthesis of covalently 2',5'-branched RNAs was not trivial by chemical methods ${ }^{176}$. Non-covalent superstructure branched RNAs in which the covalent 2',5' phosphodiester bond is missing and the branched structures were achieved by inter molecular hybridization of several RNAs have been proposed for RNA interference ${ }^{177}$. Recent advances for deoxyribozyme mediated formation of covalent 2',5'-branched RNA potentially will attract several applications.

Here we applied branch forming deoxyribozyme 10DM24 for site specific labeling of RNA molecules, using modified-GTPs as the donor substrates. Applying the same enzyme, 10DM24 on an mRNA as the scaffold (acceptor substrate) we installed adaptor RNAs (donor substrate, 19 and 33mer) and studied ribosomal translation in response to the presence of branched RNA in vitro. In another application, 6BX22 deoxyribozyme was used. 6BX22 substrate sequence requirement is compatible with formation of branched RNAs from biologically related mRNA sequences. We performed preparative scale ligation reactions (nmol) and produced study materials for investigation of interaction of U2 and U6 snRNAs and spliceosomal proteins Cwc2 and RNase H with 2', 5'- branched RNA and related RNAs.

\subsection{RNA site specific labeling}

Studying structures and dynamics of functional RNAs by biophysical techniques e.g. fluorescence spectroscopy or electron paramagnetic resonance (EPR) requires site-specific installation of spectroscopic labels. Current methods are dependent on functionalization or modification of RNA molecules by solid phase synthesis ${ }^{178}$. The functionalized RNAs undergo further reactions for installation of the reporter groups. Solid phase synthesis is reliably applicable for RNA molecules of up to 40-50 nucleotides in length. To investigate larger RNA molecules, ligation of labeled RNA fragments is required which can be performed with T4 RNA ligase, T4 DNA ligase or deoxyribozymes. 9DB1 and 7DE5 deoxyribozymes have been proven to ligate RNA fragments of G-riboswitch to synthesize its full length $^{31}$. Recently, SAM III riboswitch full length RNA has been prepared by a minimized version of 9DB1 in preparative scale ${ }^{79}$. Direct post synthetic labeling of large RNA molecules using 10DM24 deoxyribozyme is introduced here. 
10DM24 deoxyribozyme-substrate complex contains three extensive base-paired stems that form a three-helix junction (3HJ) in which the 2'-OH of the branch site becomes in close proximity to the triphosphate of the donor RNA. On the basis of presence of these stems, Höbartner and Silverman, engineered 10DM24 deoxyribozyme-substrate complex for installation of a single GTP molecule (as donor substrate) on an RNA scaffold (as acceptor substrate) ${ }^{179}$. Their result suggested that the phosphodiester bond between the most 5' and the second most 5' nucleotides of the donor RNA is unnecessary for the catalytic activity of the enzyme. Therefore, the donor RNA was dissected to a single GTP and R $\Delta$. R $\Delta$ or helper RNA sequence was identical to standard triphosphorylated donor RNA of the enzyme except that it contained a single nucleotide deletion at its 5' end and had a 5'-OH. GTP was supplied as a free donor substrate. RNA helper was aimed to maintain the RNA-DNA helix of the right binding arm of the DNA and form a binding site for the donor GTP (Fig 3-1.1). The $k_{\text {obs }}$ of GTP attachment was reported to be $0.034 \mathrm{~min}^{-1}$ in presence of $1 \mathrm{mM}$ GTP and $40 \mathrm{mM} \mathrm{Mg}^{2+}$ at $\mathrm{pH} 9.0$. Enhanced conditions i.e. $10 \mathrm{mM}$ GTP and $150 \mathrm{mM} \mathrm{Mg}^{2+}$ improved the reaction rate to $0.26 \mathrm{~min}^{-1}$.

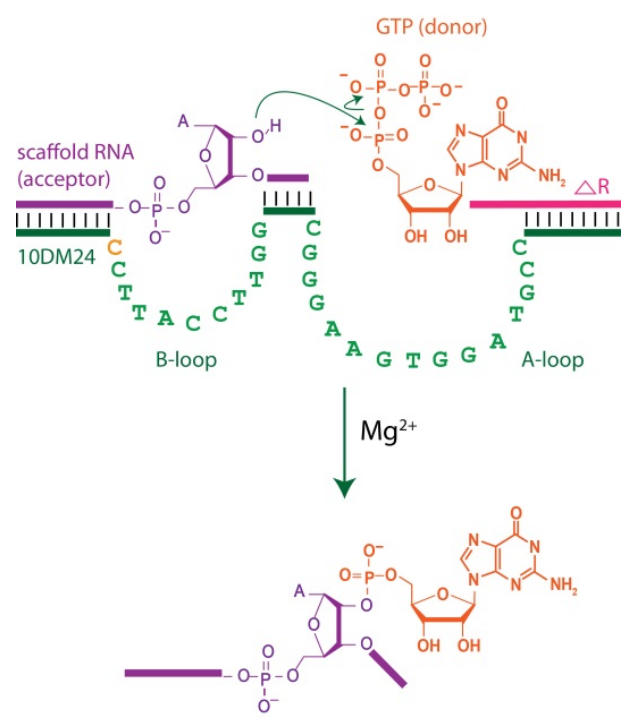

Fig 3.1-1 10DM24 catalyzed GTP attachment to RNA scaffold. The cytosine in the 3' end of the 10DM24 B loop is responsible for the recognition of the GTP. Mutation of this $\mathrm{C}$ to $\mathrm{T}$ changes the specificity of the deoxyribozyme to ligate a single ATP to the branch site.

In addition, 10DM24 was engineered to successfully attach single nucleotide ATP to the branch site of the acceptor RNA. ATP attaching 10DM24 sequence had a C to T mutation at the 3' end of the loop B of the enzyme being capable of formation of standard Watson-Crick base pairing with A. Attachment of single nucleotide CTP and UTP were not successful ${ }^{179}$.

Studies by Lea Büttner, a PhD student in our group, showed successful 10DM24 reactions with 2'modified GTPs using millimolar concentrations of (modified-) GTPs at $\mathrm{pH}$ 9.0. Employing $\mathrm{Tb}^{3+}$ mediated acceleration of the enzyme 10DM24, we improved the reaction rates as well as the GTP requirements, when performing the reaction at more neutral $\mathrm{pH}$ i.e. 7.5. Reduced $\mathrm{pH}$, reaction times and GTP requirements increases the applicability of the enzyme post-synthetic labeling of RNA molecules in preparative scales. 


\subsection{1 $\quad \mathrm{Tb}^{3+}$ assisted single GTP attachment to RNA scaffold}

In the first step, the effect of addition of $\mathrm{Tb}^{3+}$ to the GTP ligation reaction was tested. We were interested to know if the $\mathrm{Tb}^{3+}$ mediated acceleration of 10DM24 for RNA-RNA ligation (section 2.2.7) is also valid for single GTP attachment. A 16 nucleotide long RNA (R16) was chosen as standard scaffold for $\mathrm{Tb}^{3+}$ mediated acceleration of GTP attachment. In analogy to other $\mathrm{Tb}^{3+}$ mediated accelerations reported in this thesis, we performed the reactions at $\mathrm{pH} 7.5$ to avoid formation of terbium hydroxide at high $\mathrm{pH}$.

Performing single turnover kinetic reactions in presence of $10 \mathrm{mM}$ GTP and $80 \mathrm{mM} \mathrm{Mg}^{2+}$ resulted in reaction with the $k_{\mathrm{obs}} 0.02 \mathrm{~min}^{-1}$ (GTP: DNA; 10000:1). Presence of $100 \mu \mathrm{M} \mathrm{Tb}{ }^{3+}$ had slight acceleration effect and improved the $k_{\mathrm{obs}}$ only to $0.03 \mathrm{~min}^{-1}$. Surprisingly and beneficially, reducing the GTP concentration increased reaction rates. Down to a certain GTP concentration, a faster reaction could be achieved if lower concentration of GTP was present. The maximum reaction rate was in presence of 10 $\mu \mathrm{M}$ GTP with the $k_{\text {obs }} 1.6 \mathrm{~min}^{-1}$ (GTP: DNA; 10:1). Note that the GTP: DNA ratio is reduced 1000 fold and simultaneously $k_{\text {obs }}$ increased by ca. 75 fold. At lower GTP concentrations $(<10 \mu \mathrm{M})$ the reaction rates were reduced compared to the optimum conditions but still were faster than the same reaction condition in the absence of $\mathrm{Tb}^{3+}$ (Fig 3.1-2-A).

\subsubsection{Reaction scale up for $\mathrm{Tb}^{3+}$ assisted acceleration}

Preparative synthesis of labeled RNAs requires optimization of scaled up conditions. Based on kinetic data (2 pmol scaffold) $80 \mathrm{mM} \mathrm{Mg}^{2+}, 100 \mu \mathrm{M} \mathrm{Tb}^{3+}$ and $200 \mu \mathrm{M}$ GTP in $10 \mu \mathrm{l}$ was chosen as standard condition for GTP attachment. Increasing the scale (up to 500 pmol scaffold RNA) required some variation to the standard condition. For scale up optimization, the goal was to keep the amount of GTP used for each labeling reaction as standard kinetic condition i.e. 2 nmol (Table 3.1-1). Up to 250 fold increase in the scaffold RNA, reducing the DNA and RNA helper ratio to the scaffold RNA and at the same time keeping the amount of used GTP constant (2 nmol) expectedly reduced the reaction rates (Fig 3.1-2 B). Reaction conditions A-D resulted to good yields but the reaction rates were sub-optimal i.e. 70-90\% yield in 4-10 hours. Additionally, for future practical applications, long incubation times could result in RNA degradation.

An impressive improvement was achieved in condition E. The amounts for scaffold RNA, DNA, helper RNA and GTP were kept as condition B (500 pmol scaffold RNA), but the reaction volume was increased to $100 \mu$ l. Therefore, the DNA, RNA and GTP concentrations were reduced by 10 fold while $\mathrm{Mg}^{2+}$ and $\mathrm{Tb}^{3+}$ concentration were kept as standard i.e. $80 \mathrm{mM}$ and $100 \mu \mathrm{M}$ respectively. The reaction rate was enhanced ca. 400 fold in comparison to condition B (95\% product in $5 \mathrm{~min}$ ). 
Table 3.1-1 Scale-up conditions for 10DM24 catalyzed GTP attachment to model RNA. (The gel images and kinetic plots are shown in Fig 3.1-2 B).

\begin{tabular}{ccccccccccc} 
Condition & $\begin{array}{c}\text { Scaffold } \\
(\mathrm{pmol})\end{array}$ & $\begin{array}{c}\text { vol. } \\
(\mu \mathrm{l})\end{array}$ & $\begin{array}{c}\text { Scaffold: DNA: helper } \\
(\mu \mathrm{M})\end{array}$ & $\begin{array}{c}{[\mathrm{GTP}]} \\
(\mu \mathrm{M})\end{array}$ & $\begin{array}{c}\mathrm{GTP} \\
(\mathrm{nmol})\end{array}$ & $\begin{array}{c}\mathrm{Mg}^{2+} \\
(\mathrm{mM})\end{array}$ & $\begin{array}{c}\mathrm{Tb}^{3+} \\
(\mu \mathrm{M})\end{array}$ & $\begin{array}{c}\mathrm{Tb}^{3+} / \mathrm{DNA}^{2} \\
\mathrm{~Tb}^{3+} / \mathrm{GTP}\end{array}$ & $\begin{array}{c}k_{\mathrm{obs}} \\
\left(\mathrm{min}^{-1}\right)\end{array}$ \\
\hline kinetic scale & 2 & 10 & $0.2: 1: 2$ & 200 & 2 & 80 & 100 & 100 & 0.5 & 0.9 \\
A & 200 & 10 & $20: 30: 50$ & 200 & 2 & 80 & 100 & 3.3 & 0.5 & 0.007 \\
B & 500 & 10 & $50: 60: 80$ & 200 & 2 & 80 & 100 & 1.6 & 0.5 & 0.004 \\
C & 500 & 10 & $50: 60: 80$ & 200 & 2 & 80 & 200 & 3.3 & 1 & 0.016 \\
D & 500 & 10 & $50: 60: 80$ & 400 & 4 & 80 & 200 & 3.3 & 0.5 & 0.018 \\
E & 500 & 100 & $5: 6: 8$ & 20 & 2 & 80 & 100 & 16 & 5 & 1.6
\end{tabular}

Surprisingly, the reaction rate was higher when GTP concentration was reduced by 10 -fold i.e. from 200 to $20 \mu \mathrm{M}$. We hypothesized that the ratio of metal ions, $\mathrm{Mg}^{2+}$ and $\mathrm{Tb}^{3+}$ to oligonucleotides is the crucial factor. In standard condition for kinetic studies $\mathrm{Tb}^{3+}$ concentration was 100-fold in excess to DNA. The $\mathrm{Tb}^{3+} / \mathrm{DNA}$ ratios in condition A-D with reduced rates were below 4. In condition $\mathrm{E}$ with high reaction rate the $\mathrm{Tb}^{3+} / \mathrm{DNA}$ was 16 . Our dataset is not sufficient to make final conclusive statement that the ratio of which of the metal ions to which of the oligonucleotides (scaffold, DNA or helper RNA) is important, but taking $\mathrm{Tb}^{3+}$ as the representative of metal ions and DNA as the representative of oligonucleotides we concluded that as a rule of thumb, $\mathrm{Tb}^{3+} / \mathrm{DNA}$ ratio must be above 10 .
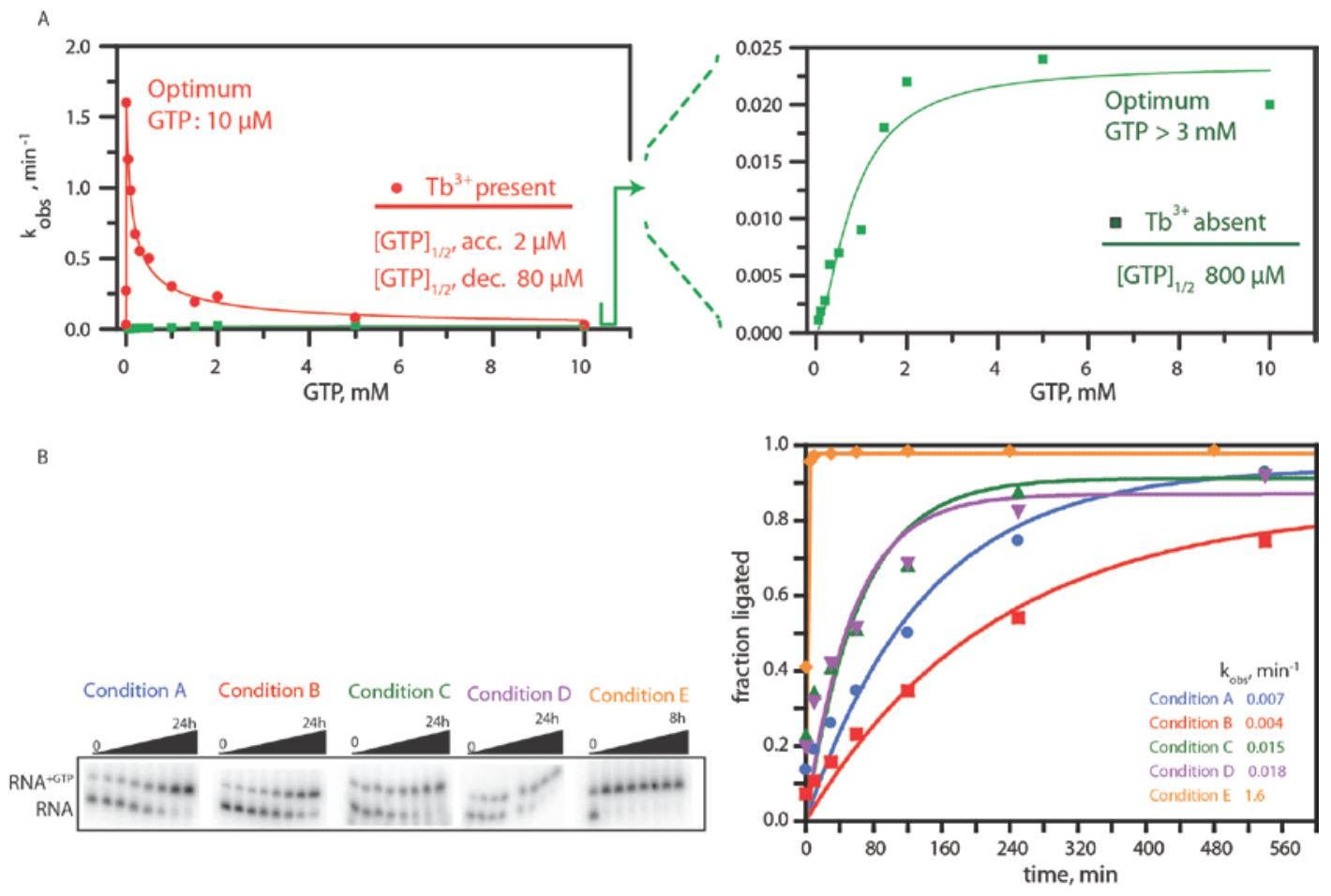

Fig 3.1-2 A) 10DM24 GTP requirement in the presence and absence of optimal $\mathrm{Tb}^{3+}$. Reactions were performed in kinetic scale for 2 pmol scaffold RNA: 10 pmol DNA: 20 pmol helper RNA in $10 \mu l$. The right panel is the zoomed view of the left panel for the $k_{\mathrm{obs}}$ of the reactions in the absence of $\mathrm{Tb}^{3+}$.B) Scale-up reactions for GTP attachment to 500 pmol scaffold RNA. The conditions are explained in detail in table 3.1-1. Time points for kinetic reaction A-D: 1, 10, 30 min, 1, 2, 4, 9, $24 \mathrm{~h}$. For reaction E: 0, 5, 10, $30 \mathrm{~min}, 1,2,4,8 \mathrm{~h}$. The best condition (E): scaffold: 10DM24: helper RNA 5:6:8 $\mu \mathrm{M}$, GTP $20 \mu \mathrm{M}, \mathrm{MgCl}_{2} 80 \mathrm{mM}, \mathrm{TbCl}_{3} 100 \mu \mathrm{M}$, reaction volume $100 \mu \mathrm{l}$. 
The same dataset suggests that the determining factor to achieve fast reactions is not the ratio of metal ions to GTP. The $\mathrm{Tb}^{3+} / \mathrm{GTP}$ ratio was in the same range (0.5-1) in fast standard kinetic reaction and reaction conditions A-D which were slower. In the other comparing standard condition and condition $\mathrm{E}$ which both have fast reactions, the $\mathrm{Tb}^{3+} / \mathrm{GTP}$ ratio is different ( 0.5 and 5 folds).

\subsubsection{The effect of mutations adjacent to the branch site on GTP attachment}

For General application of 10DM24 for RNA labeling we aimed to investigate the effect of neighbor positions adjacent to the branch site on GTP attachment rate and yield. We comprehensively studied kinetics of single and double mutations of the scaffold RNA (R16) in four different conditions. The enzyme binding arm was co-mutated to be complementary with the mutant substrates. We studied all 16 possible combination of $\mathrm{N} \underline{\mathrm{AN}}$ (N: any nucleotide, $\underline{\mathrm{A}}$ : branch site A) at four conditions; condition I: RNA helper, pH 9.0, 3 mM GTP, $100 \mathrm{mM} \mathrm{Mg}^{2+}$, condition II: RNA helper, pH 7.5, $200 \mu \mathrm{M}$ GTP, 80 $\mathrm{mM} \mathrm{Mg}^{2+}$, Condition III: RNA helper, pH 7.5, $200 \mu \mathrm{M} \mathrm{GTP,} 80 \mathrm{mM} \mathrm{Mg}^{2+}$ with $100 \mu \mathrm{M} \mathrm{Tb}^{3+}$, Condition

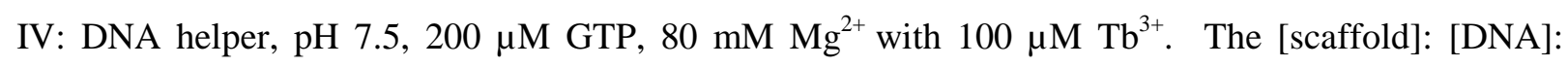
[helper] was 2:10:20 $\mu \mathrm{M}$ for all conditions (Fig 3.1-3). The reaction rates, extrapolated yields and actual yields for each mutation and condition are shown in the table 3.1-2.
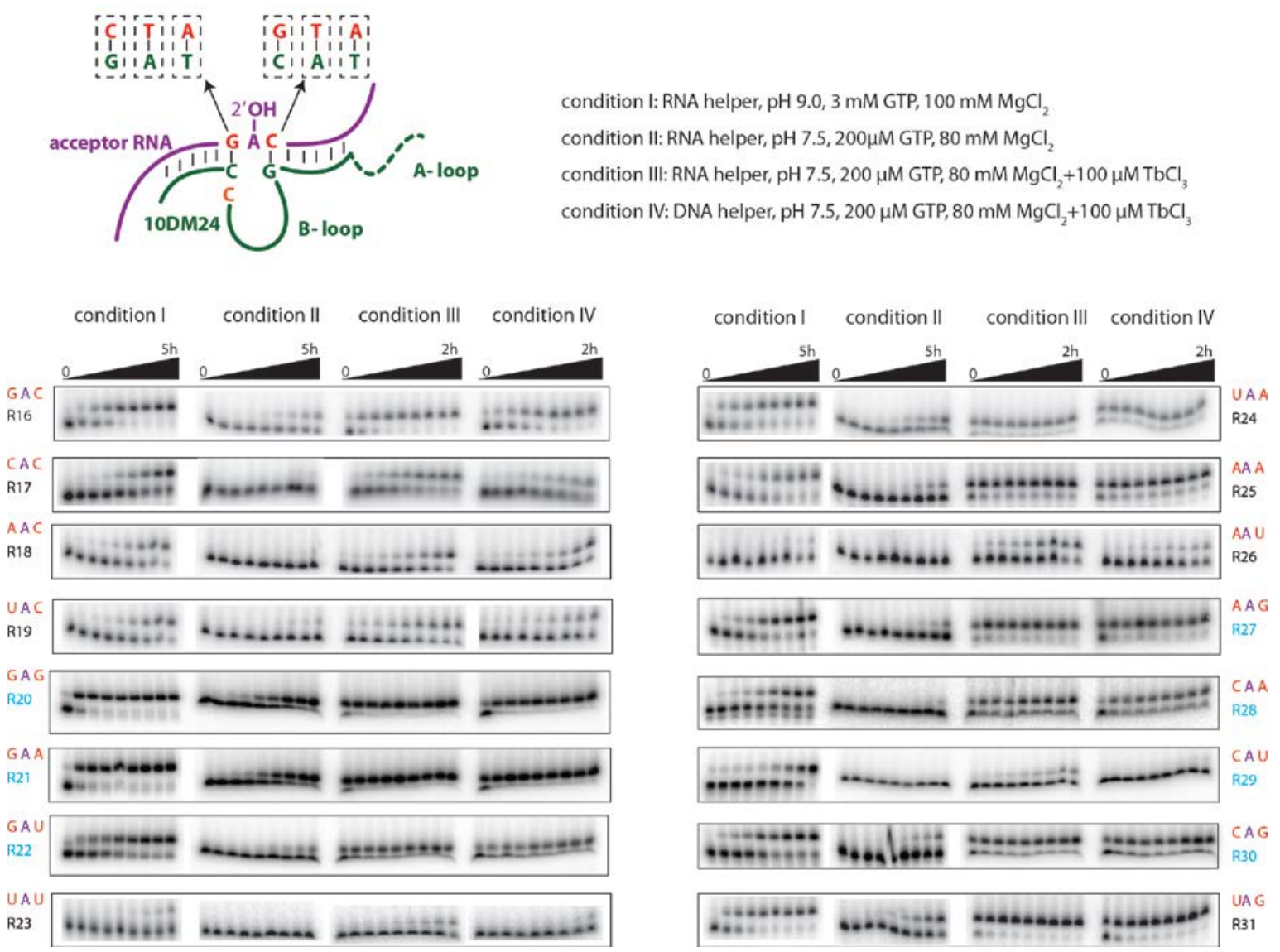

Fig 3.1-3 Kinetic studies for scaffold mutants at the branch site. The 10DM24 binding arms were co-mutated according to the RNA substrate to maintain base pairings (shown schematically on top). Time points (I and II): 15 sec, 2, 5, 10, 30, 60, 120, 180, $300 \mathrm{~min}$. Times points (III and IV): $15 \mathrm{sec}, 1$, 2, 5, 10, 20, 30, 60, $120 \mathrm{~min}$. The kinetic data marked with blue were performed by Lea Büttner. 
Comparing condition II and III reveals the $\mathrm{Tb}^{3+}$ mediated acceleration effect for each mutant. As expected very slow reactions were observed at condition II. Addition of $\mathrm{Tb}^{3+}$ accelerates the reaction by a factor of 80-1600-fold. The lowest and highest acceleration factors were for sequence context AAC and AAA respectively. In special cases as UAU, CAU and AAU, there was no product formed in the absence of $\mathrm{Tb}^{3+}$ at the given time and acceleration upon presence of $\mathrm{Tb}^{3+}$ only had a minor effect for formation of the product at the given time. As the product is formed in all the mutation combinations (although for the three special cases with reduced yield), no mutation around the branch site was detrimental for catalysis.

The first report for 10DM24 mediated GTP attachment have employed an RNA sequence as helper oligonucleotide to hybridize to the right binding arm of the DNA. Including a DNA helper in our mutant studies (condition IV) confirmed no difference between the RNA and DNA helper for the efficiency of the reaction.

Table 3.1-2 Rate and yield of mutant RNAs for GTP attachment. The model RNA and single point mutants relative to it are marked peach. In each table the rows and columns indicate the identity of the 5' and 3' adjacent positions respectively. In each cell of the tables the second line indicates the $k_{\text {obs }}, \min ^{-1}$ and the maximum extrapolated yield. The third row indicates the actual observed yield at the indicated time point. The data marked in blue were obtained by Lea Büttner. The $k_{\text {obs }}$ values marked with * are from linear fit. The reactions with the rate higher than 0.5 min $^{-1}$ are marked with **.

condition I: RNA helper, CHES pH 9.0, 3 mM GTP, $100 \mathrm{mM} \mathrm{MgCl}$

\begin{tabular}{|c|c|c|c|c|}
\hline & G & $\mathrm{A}$ & $\mathrm{C}$ & $\mathrm{U}$ \\
\hline $\mathrm{G}$ & $\begin{array}{l}\text { R20 } \\
(0.745,96 \%) \\
(99 \% \text { in } 5 \mathrm{~h})\end{array}$ & $\begin{array}{l}\text { R21 } \\
(0.620,93 \%) \\
(96 \% \text { in } 5 \mathrm{~h})\end{array}$ & $\begin{array}{l}\text { R16 } \\
(0.103,93 \%) \\
(96 \% \text { in } 5 \mathrm{~h})\end{array}$ & $\begin{array}{l}\text { R22 } \\
(0.109,97 \%) \\
(100 \% \text { in } 5 \mathrm{~h})\end{array}$ \\
\hline A & $\begin{array}{l}\text { R27 } \\
(0.033,82 \%) \\
(80 \% \text { in } 20 \mathrm{~h})\end{array}$ & $\begin{array}{l}\text { R25 } \\
(0.031,79 \%) \\
(82 \% \text { in } 5 \mathrm{~h})\end{array}$ & $\begin{array}{l}\text { R18 } \\
(0.007,85 \%) \\
(76 \% \text { in } 5 \mathrm{~h})\end{array}$ & $\begin{array}{l}\text { R26 } \\
(0.004 *) \\
(76 \% \text { in } 24 h)\end{array}$ \\
\hline $\mathrm{C}$ & $\begin{array}{l}\mathrm{R} 30 \\
(0.031,74 \%) \\
(81 \% \text { in } 20 \mathrm{~h})\end{array}$ & $\begin{array}{l}\text { R28 } \\
(0.014,74 \%) \\
(80 \% \text { in } 20 \mathrm{~h})\end{array}$ & $\begin{array}{l}\text { R17 } \\
(0.011,72 \%) \\
(76 \% \text { in } 24)\end{array}$ & $\begin{array}{l}\text { R29 } \\
\left(0.057^{*}\right) \\
(94 \% \text { in } 5 \mathrm{~h})\end{array}$ \\
\hline $\mathrm{U}$ & $\begin{array}{l}\text { R31 } \\
(0.193,90 \%) \\
(92 \% \text { in } 5 \mathrm{~h})^{* *}\end{array}$ & $\begin{array}{l}\text { R24 } \\
(0.188,78 \%) \\
(84 \% \text { in } 5 \mathrm{~h})\end{array}$ & $\begin{array}{l}\text { R19 } \\
(0.029,78 \%) \\
(85 \% \text { in } 24)\end{array}$ & $\begin{array}{l}\text { R23 } \\
(0.001,80 \%) \\
(70 \% \text { in } 24)\end{array}$ \\
\hline
\end{tabular}

condition II: RNA helper, HEPES pH 7.5, $200 \mu \mathrm{M}$ GTP, $80 \mathrm{mM} \mathrm{MgCl}{ }_{2}$

\begin{tabular}{|c|c|c|c|c|}
\hline & G & $\mathrm{A}$ & $\mathrm{C}$ & $\mathrm{U}$ \\
\hline G & $\begin{array}{l}\text { R20 } \\
(0.013,96 \%) \\
(96 \% \text { in } 20 \mathrm{~h})\end{array}$ & $\begin{array}{l}\text { R21 } \\
(0.014,91 \%) \\
(94 \% \text { in } 20 \mathrm{~h})\end{array}$ & $\begin{array}{l}\text { R16 } \\
(0.004,63 \%) ; \\
(46 \% \text { in } 5 \mathrm{~h})\end{array}$ & $\begin{array}{l}\mathrm{R} 22 \\
(0.004,60 \%) \\
(62 \% \text { in } 20 \mathrm{~h})\end{array}$ \\
\hline A & $\begin{array}{l}\text { R27 } \\
(0.003,31 \%) \\
(30 \% \text { in } 20 \mathrm{~h})\end{array}$ & $\begin{array}{l}\text { R25 } \\
(0.003,40 \%) \\
(25 \% \text { in } 5 \mathrm{~h})\end{array}$ & $\begin{array}{l}\text { R18 } \\
\left(0.0002^{*}\right) \\
(5 \% \text { in } 5 \mathrm{~h})\end{array}$ & $\begin{array}{l}\text { R26 } \\
(0.0001 *) \\
(2 \% \text { in } 5 \mathrm{~h})\end{array}$ \\
\hline $\mathrm{C}$ & $\begin{array}{l}\text { R30 } \\
(0.003,36 \%) \\
(35 \% \text { in } 20 \mathrm{~h})\end{array}$ & $\begin{array}{l}\text { R28 } \\
\left(0.007^{*}\right) \\
(54 \% \text { in } 20 \mathrm{~h})\end{array}$ & $\begin{array}{l}\text { R17 } \\
\left(0.00012^{*}\right) \\
(8 \% \text { in } 5 \mathrm{~h})\end{array}$ & $\begin{array}{l}\text { R29 } \\
\left(0.0002^{*}\right) \\
(5 \% \text { in } 5 \mathrm{~h})\end{array}$ \\
\hline $\mathrm{U}$ & $\begin{array}{l}\text { R31 } \\
(0.004,82 \%) \\
(59 \% \text { in } 5 \mathrm{~h})\end{array}$ & $\begin{array}{l}\text { R24 } \\
(0.005,72 \%) \\
(61 \% \text { in } 5 \mathrm{~h})\end{array}$ & $\begin{array}{l}\text { R19 } \\
\left(0.0001^{*}\right) \\
(10 \% \text { in } 5 \mathrm{~h})\end{array}$ & $\begin{array}{l}\text { R23 } \\
(0.00001 *) \\
(9 \% \text { in } 5 \mathrm{~h})\end{array}$ \\
\hline
\end{tabular}

Condition III: RNA helper, HEPES pH 7.5, $200 \mu \mathrm{M} \mathrm{GTP}, 80 \mathrm{mM} \mathrm{MgCl}_{2}+100 \mu \mathrm{M} \mathrm{TbCl}_{3}$

\begin{tabular}{|c|c|c|c|c|}
\hline & $\mathrm{G}$ & $\mathrm{A}$ & $\mathrm{C}$ & $\mathrm{U}$ \\
\hline G & $\begin{array}{l}\text { R20 } \\
(2.31,97 \%) \\
(100 \% \text { in } 2 \mathrm{~h})^{* *}\end{array}$ & $\begin{array}{l}\text { R21 } \\
(2.68,93 \%) \\
(99 \% \text { in } 2 \mathrm{~h})^{* * *}\end{array}$ & $\begin{array}{l}\text { R16 } \\
(0.914,93 \%) \\
(95 \% \text { in } 2 \mathrm{~h})\end{array}$ & $\begin{array}{l}\mathrm{R} 22 \\
(1.60,71 \%) \\
(77 \% \text { in } 2 \mathrm{~h})^{* *}\end{array}$ \\
\hline A & $\begin{array}{l}\text { R27 } \\
(2.28,87 \%) \\
(91 \% \text { in } 2 \mathrm{~h})^{* *}\end{array}$ & $\begin{array}{l}\mathrm{R} 25 \\
(5.3,82 \%) \\
(85 \% \text { in } 2 \mathrm{~h})^{* *}\end{array}$ & $\begin{array}{l}\mathrm{R} 18 \\
(0.016,84 \%) \\
(74 \% \text { in } 2 \mathrm{~h})\end{array}$ & $\begin{array}{l}\text { R26 } \\
(0.038,59 \%) \\
(61 \% \text { in } 2 \mathrm{~h})\end{array}$ \\
\hline $\mathrm{C}$ & $\begin{array}{l}\text { R30 } \\
(1.61,87 \%) \\
(85 \% \text { in } 2 \mathrm{~h})^{* *}\end{array}$ & $\begin{array}{l}\text { R28 } \\
(1.85,63 \%) \\
(73 \% \text { in } 2 \mathrm{~h})^{* *}\end{array}$ & $\begin{array}{l}\text { R17 } \\
(0.167,68 \%) \\
(74 \% \text { in } 2 \mathrm{~h})\end{array}$ & $\begin{array}{l}\text { R29 } \\
\left(0.020^{*}\right) \\
(77 \% \text { in } 20 \mathrm{~h})\end{array}$ \\
\hline$\overline{\mathrm{U}}$ & $\begin{array}{l}\text { R31 } \\
(6.0,95 \%) \\
(97 \% \text { in } 2 \mathrm{~h})^{* *}\end{array}$ & $\begin{array}{l}\text { R24 } \\
(7.8,83 \%) \\
(87 \% \text { in } 2 \mathrm{~h})^{* * *}\end{array}$ & $\begin{array}{l}\text { R19 } \\
(0.082,66 \%) \\
(69 \% \text { in } 2 \mathrm{~h})\end{array}$ & $\begin{array}{l}\text { R23 } \\
(0.030,38 \%) \\
(40 \% \text { in } 2 \mathrm{~h})\end{array}$ \\
\hline
\end{tabular}

condition IV: DNA helper, HEPES pH 7.5, $200 \mu \mathrm{M} \mathrm{GTP}, 80 \mathrm{mM} \mathrm{MgCl}_{2}+100 \mu \mathrm{M} \mathrm{TbCl}_{3}$

\begin{tabular}{|c|c|c|c|c|}
\hline & G & $\mathrm{A}$ & $\mathrm{C}$ & $\mathrm{U}$ \\
\hline G & $\begin{array}{l}\text { R20 } \\
(1.86,94 \%) \\
(98 \% \text { in } 2 \mathrm{~h})^{* *}\end{array}$ & $\begin{array}{l}\text { R21 } \\
(2.41,92 \%) \\
(98 \% \text { in } 2 \mathrm{~h})^{* *}\end{array}$ & $\begin{array}{l}\text { R16 } \\
(0.223,88 \%) \\
(91 \% \text { in } 2 \mathrm{~h})\end{array}$ & $\begin{array}{l}\mathrm{R} 22 \\
(1.03,65 \%) \\
(75 \% \text { in } 2 \mathrm{~h})^{* *}\end{array}$ \\
\hline A & $\begin{array}{l}\text { R27 } \\
(2.01,86 \%) \\
(91 \% \text { in } 2 \mathrm{~h})^{* *}\end{array}$ & $\begin{array}{l}\text { R25 } \\
(4.5,79 \%) \\
(82 \% \text { in } 2 \mathrm{~h})^{* *}\end{array}$ & $\begin{array}{l}\text { R18 } \\
(0.013,82 \%) \\
(65 \% \text { in } 2 \mathrm{~h})\end{array}$ & $\begin{array}{l}\mathrm{R} 26 \\
(0.027,35 \%) \\
(34 \% \text { in } 2 \mathrm{~h})\end{array}$ \\
\hline $\mathrm{C}$ & $\begin{array}{l}\text { R30 } \\
(1.48,74 \%) \\
(82 \% \text { in } 2 \mathrm{~h})^{* *}\end{array}$ & $\begin{array}{l}\text { R28 } \\
(1.61,62 \%) \\
(69 \% \text { in } 2 \mathrm{~h})^{* *}\end{array}$ & $\begin{array}{l}\text { R17 } \\
(0.029,56 \%) \\
(57 \% \text { in } 2 \mathrm{~h})\end{array}$ & $\begin{array}{l}\text { R29 } \\
\left(0.035^{*}\right) \\
(7 \% \text { in } 2 \mathrm{~h})\end{array}$ \\
\hline $\mathrm{U}$ & $\begin{array}{l}\text { R31 } \\
(2.7,93 \%) \\
(97 \% \text { in } 2 \mathrm{~h})^{* *}\end{array}$ & $\begin{array}{l}\text { R24 } \\
(6.5,78 \%) \\
(79 \% \text { in } 2 h)^{* *}\end{array}$ & $\begin{array}{l}\text { R19 } \\
(0.019,54 \%) \\
(50 \% \text { in } 2 \mathrm{~h})\end{array}$ & $\begin{array}{l}\text { R23 } \\
(0.14,15 \%) \\
(21 \% \text { in } 2 \mathrm{~h})\end{array}$ \\
\hline
\end{tabular}


According to the mutation data, condition I led to high product yield within 5h incubation at $\mathrm{pH} 9.0$ in the presence of $100 \mathrm{mM} \mathrm{Mg}^{2+}$ and $3 \mathrm{mM}$ GTP for all sequence contexts except AAU and UAU (Fig 3.15 B). Long incubation time at high $\mathrm{pH}$ did not result in any degradation of the RNA model (R16, 16nt). However, to apply the method for biologically relevant longer RNAs condition I can cause degradation of RNA substrate. In the other hand, for further applications, using $3 \mathrm{mM}$ modified-GTP for RNA labeling might not be affordable. In contrast, condition III with only $200 \mu \mathrm{M}$ GTP and at pH 7.5 reached more than $50 \%$ product in $10 \mathrm{~min}$ for 10 out of all 16 possible sequence contexts. At the same incubation time $(10 \mathrm{~min})$ condition I led to more than $50 \%$ reaction product only for 5 sequence contexts (Fig 3.1-4 A and C).
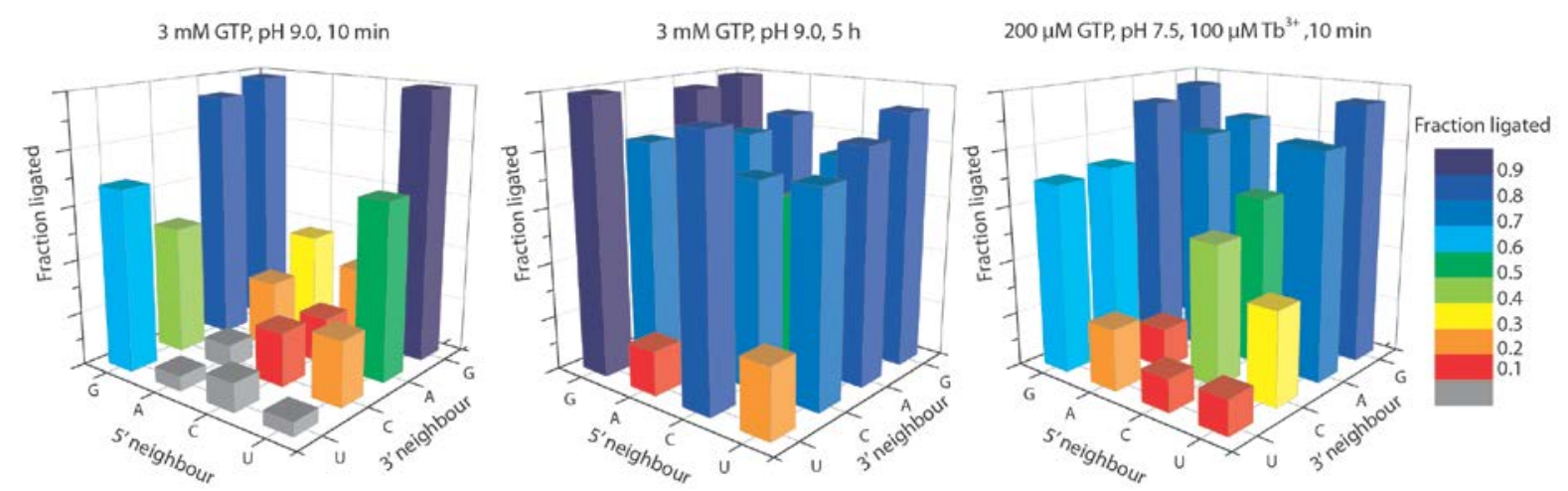

Fig 3.1-4 Ligated fractions for all 16 sequence contexts around the adenosine branch site of R16 at conditions I (3 mM GTP, $100 \mathrm{mM} \mathrm{Mg}^{2+}$ at pH 9.0) and III (200 $\mathrm{\mu M} \mathrm{GTP,} 80 \mathrm{mM} \mathrm{Mg} \mathrm{Mg}^{2+}, 100 \mu \mathrm{M} \mathrm{Tb^{3+ }}$ at pH 7.5). Data are shown for $10 \mathrm{~min}$ and $5 \mathrm{~h}$ of condition $\mathrm{I}$ and for $10 \mathrm{~min}$ at condition III. At both conditions purin embeded adenosines are better ligated. Importantly, comparing $10 \mathrm{~min}$ data points, ligations in presence of $\mathrm{Tb}^{3+}$ and at lower GTP concentration are more efficient.

For further development of the method, condition III was chosen. Based on the mutation data it is predictable that in any given RNA sequence adenosines with purine neighbors at 5' or 3' or both sides are most accessible for GTP attachment and adenosines with 3' uridine neighbor should be avoided except if 5' neighbor is a guanosine.

In addition to the standard 16 nucleotide long RNA model, condition III was employed to attach GTP for 22 adenosines at different RNA models ranging from 22-112 nucleotide long. Fig 3.1-5 summarizes the reaction rates and yield at $15 \mathrm{~min}$ and $3 \mathrm{~h}$ for model RNAs which were shorter than 55 nucleotide long. 
Fig 3.1-5 GTP attachment to model RNAs applying condition III (200 $\mu \mathrm{M} \mathrm{GTP,} 80 \mathrm{mM} \mathrm{Mg^{2+ } , 1 0 0 \mu M} \mathrm{Tb}^{3+}$ at $\left.\mathrm{pH} 7.5\right)$. The target branch site and the sequence context are marked on top of each gel, time points for $24 \mathrm{~h}$ reactions were 15 sec $2,5,15,30 \mathrm{~min}, 1,2,3,24 \mathrm{~h}$, time points for $5 \mathrm{~h}$ reactions were $15 \mathrm{sec}, 2,5,15,30 \mathrm{~min}, 1$, 2,3 ,5 h.

One of the RNA models in Fig 3.1-5 was a short fragment of U6-snRNA including positions 30-83 (GenBank: X12565.1). U6-snRNA fragment was included since site specific labeling of snRNAs with fluorophors or paramagnetic labels would facilitate in vitro studies of ribonucleoproteins (RNP) complexes. RNPs play essential roles in eukaryotic cellular processes related to gene expression i.e. splicing of mRNAs or maturation of tRNAs, nuclear transport and translation ${ }^{88}$. GTP attachment to short U6-snRNA revealed positions with high yield for GTP attachment. Attachment of GTP to full length U6-snRNA (112 nucleotide long) requires further method developments for separation of the GTP labeled long RNA since the molecular weight of a single GTP is not sufficient to differentiate between labeled and non-labeled RNAs as such lengths (discussed in section 3.1.8).

\subsubsection{Labeling of RNA with modified NTP in kinetic scale}

Previous studies in our research group for attachment of modified GTPs revealed that the enzyme 10DM24 accepts GTP modifications when the modification is placed at the 2' position. Here we proved that $\mathrm{Tb}^{3+}$ mediated acceleration of GTP attachment is valid for 2'-modified GTPs. EDA linked Biotin, TAMRA and Cy3 GTPs were successfully attached to the model RNA. The reaction for GTP-EDATAMRA was slower ( $k_{\mathrm{obs}}$ : $0.003-0.004 \mathrm{~min}^{-1}$ ) but reached more than $65 \%$ yield in $24 \mathrm{~h}$. Mant-GTP attachment to R016 was successful as well $\left(k_{\text {obs: }} 0.07 \mathrm{~min}^{-1}\right)$. Mant modification of GTP was via an ester bond and survived in the course of reaction. At later time points, an extra band was observed below the Mant-GTP ligated product which was deduced to be the hydrolyzed form of the GTP missing the Mant modification. The products ligated to the modified GTPs showed larger gel retardation in denaturing 
PAGE in comparison to non-modified GTP suggesting presence of the modification at the attached GTP (Fig 3.1-6 A).

ATP attachment to RNA model (R16) in presence of standard condition (Condition III) showed similar reaction rate to reported value for ATP attachment in the presence of $1 \mathrm{mM} \mathrm{ATP,} 40 \mathrm{mM} \mathrm{MgCl} 2$ at $\mathrm{pH}$ 9.0. Despite successful ATP attachment to R016, attachment of ATP-EDA-Cy5 at condition III was very poor (2\% in 4 h, Fig 3.1-6 B).

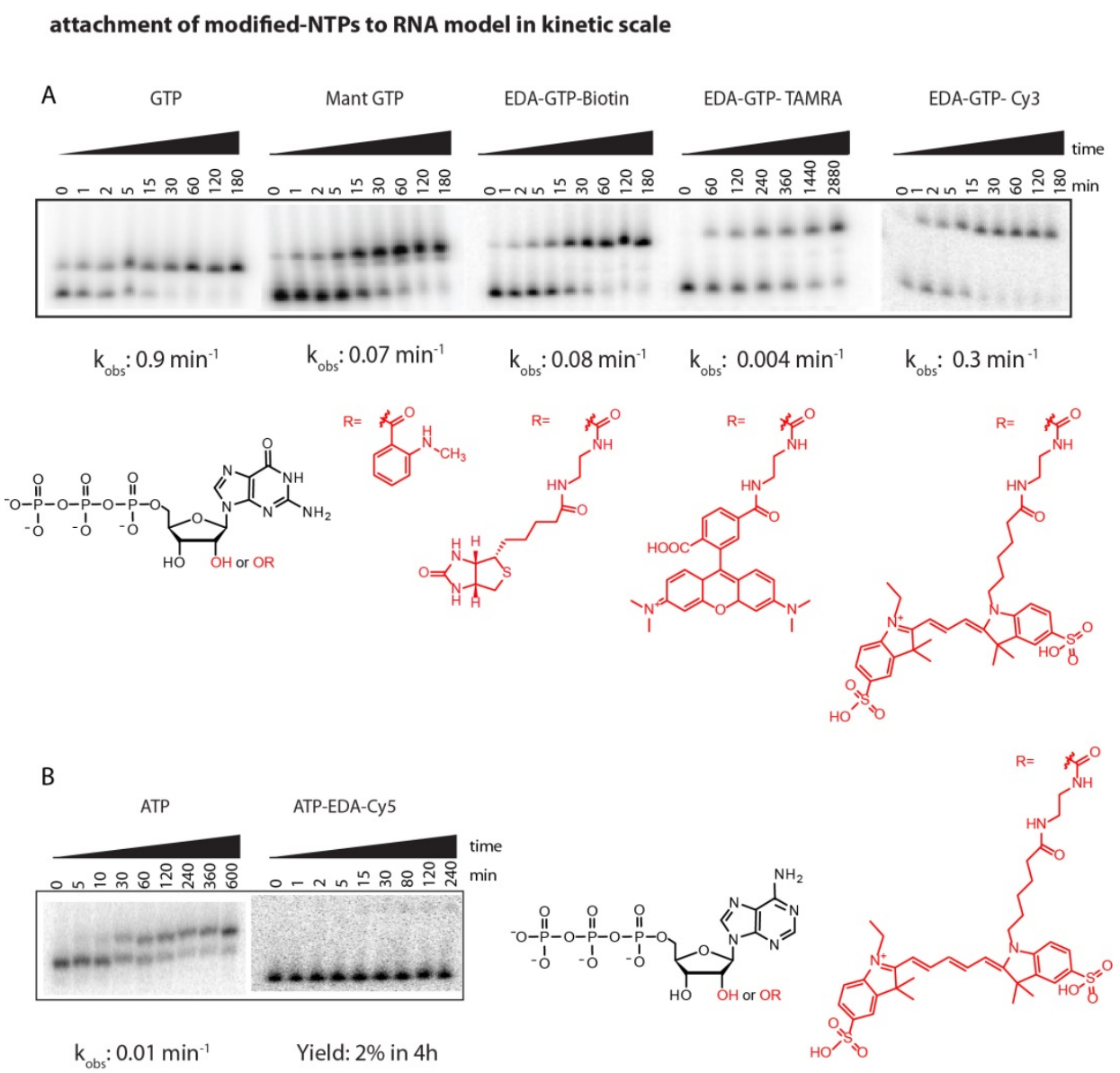

Fig 3.1-6 Attachment of (modified-) NTPs to model RNA (R16) using condition III of Fig 3.1-3 i.e. $200 \mu M$ (modified-) NTP, $80 \mathrm{mM} \mathrm{Mg}^{2+}$ and $100 \mu \mathrm{M} \mathrm{Tb^{3+ }}$. A) Attachment of GTP and modified GTPs to RNA model. B) Attachment of ATP and ATP-EDA-Cy5 to RNA model.

\subsubsection{Labeling of RNA with modified GTPs in preparative scale}

To confirm resistance of the EDA and ester bond during the labeling reaction and gel purifications, preparative reactions were performed using a 26 nucleotide long RNA model containing identical sequence to the 3' of the SAM III riboswitch from its position 28 to 53 (Tr18). Exploiting GTP attaching enzyme for position A44 of SAM III (D121), attachment of modified- GTPs Tr18 was investigated.

As it was observed in kinetic scale, attachment of (modified)-GTP resulted in further shift in the denaturing gel. The larger gel retardation confirmed persistence of the modification in the reaction 
condition and during gel electrophoresis. Fig 3.1-7 A shows the preparative scale attachment of modified GTPs to Tr18. The product bands which are marked with red stars were cut and extracted. The isolated yield of the labeled Tr18 is indicated below each reaction. In Fig 3.1-7 B, 100 pmol scale reactions were performed and loaded directly besides $100 \mathrm{pmol}$ of the purified products from gels in Fig 3.1-7 A. The result confirmed that the modifications persist the reaction condition and gel electrophoresis and also they were stable during purification steps.

For further confirmations gel purified Mant and TAMRA labeled Tr18 were subjected for fluorescence measurements. The fluorescent data (Fig 3.1-8 A) confirmed that the EDA linked TAMRA and ester linked Mant modification survived the reaction conditions and are present with the extracted RNA sample. These results do not necessarily confirm that the EDA linkage is not hydrolyzed during gel extraction steps.

The same samples, in addition to biotin-EDA-GTP labeled Tr18 (gel purified) were analyzed by anion exchange HPLC (Fig 3.1-8 B). Non-modified Tr18 had retention time at $16.8 \mathrm{~min}$. Gel purified GTP and Mant-GTP modified Tr18 retention times were 17.3 and 19.6 min respectively. For Mant-GTP modified Tr18 an additional peak at 17.3 min was observed. This peak was deduced to be the hydrolyzed form of the attached GTP as was also observed in denaturing PAGE. Although, EDA linked modifications, TAMRA and Biotin retention times were $17.3 \mathrm{~min}$, similar to non-modified GTP, gel shifts confirmed presence of a modified GTP in the case of Biotin-EDA-GTP and TAMRA-EDA-GTP. Also, fluorescent data was in agreement to presence of TAMRA on the purified TAMRA-EDA-GTP ligated $\operatorname{Tr} 18$.

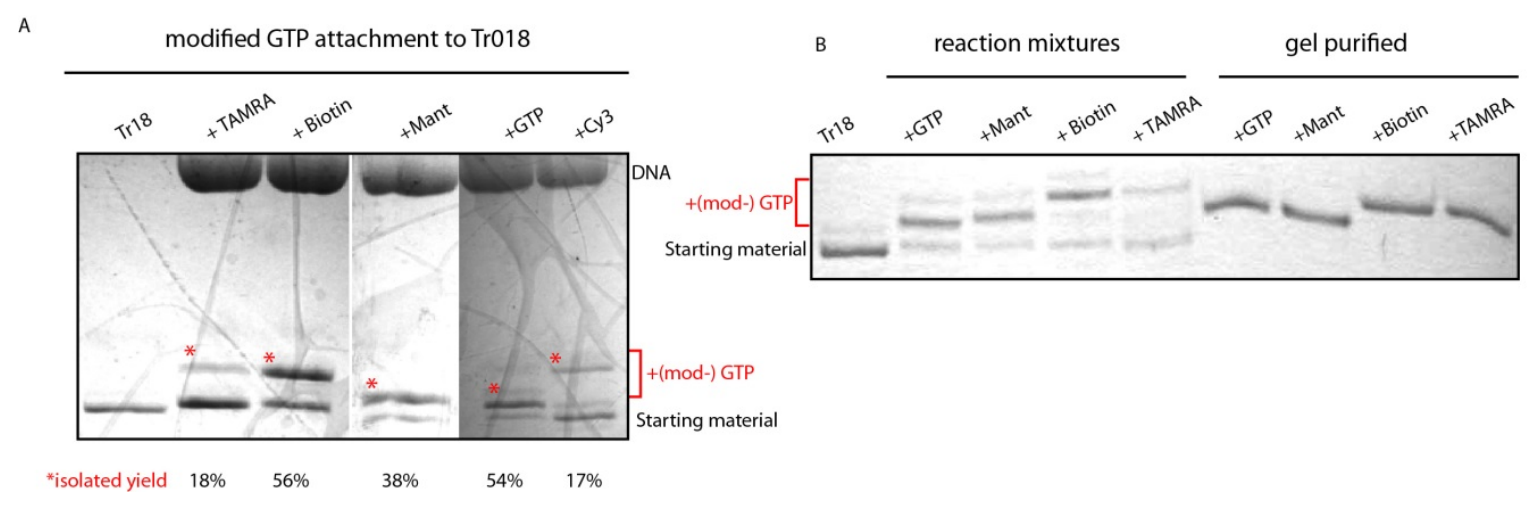

Fig 3.1-7 Preparative attachment of modified GTP. A) reactions were performed for $5 \mu \mathrm{MNA}$ in presence of $80 \mathrm{mM}$ $\mathrm{MgCl}_{2}, 100 \mu \mathrm{M} \mathrm{TbCl}, \mathrm{pH} 7.5,30 \mathrm{~min}$ at $37^{\circ} \mathrm{C}$ with $20 \mu \mathrm{M}$ modified GTP in $100 \mu \mathrm{l}$. Samples were ethanol precipitated and loaded on 15\% gel, $0.4 \mathrm{~mm}$ thick, $1 \mathrm{nmol}$ per lane. Gel electrophoresis was performed at $35 \mathrm{~W}$ for $1 \mathrm{~h} 30$. B) purified (modified-) GTP labeled RNAs of A are loaded besides the reaction mixtures of (modified-) GTP attachment to Tr18. 100 pmol samople was loaded on each lane. The gel was stained by tuloidine blue.

It can be hypothesized that either the EDA linkage does not survive the high temperature during HPLC chromatography or that such conditions for HPLC are not suitable to resolve the presence of TAMRA and Biotin modification. The first hypothesis can be excluded as TAMRA-modified RNA showed an 
absorbance at $545 \mathrm{~nm}$ (signature for TAMRA) at the same retention time as the peak at $260 \mathrm{~nm}$ (17.3 min, Fig 3.1-8 B). As a control experiment the reaction mixtures for attachment of TAMRA-EDA-GTP, Biotin-EDA-GTP and non- modified GTP (100 pmol scale) directly, skipping the gel purification step, injected on HPLC. The same retention times were observed for all three cases. In summary, it can be concluded that the EDA linked modifications were accepted to be attached at large scale to RNA and the EDA linkage was tolerated under $\mathrm{Tb}^{3+}$ mediated acceleration condition, gel electrophoresis, gel purification and HPLC but HPLC is not suitable to resolve the presence of TAMRA and biotin on Tr18. Additional confirmation by Mass spectroscopy is required to confirm this hypothesis.

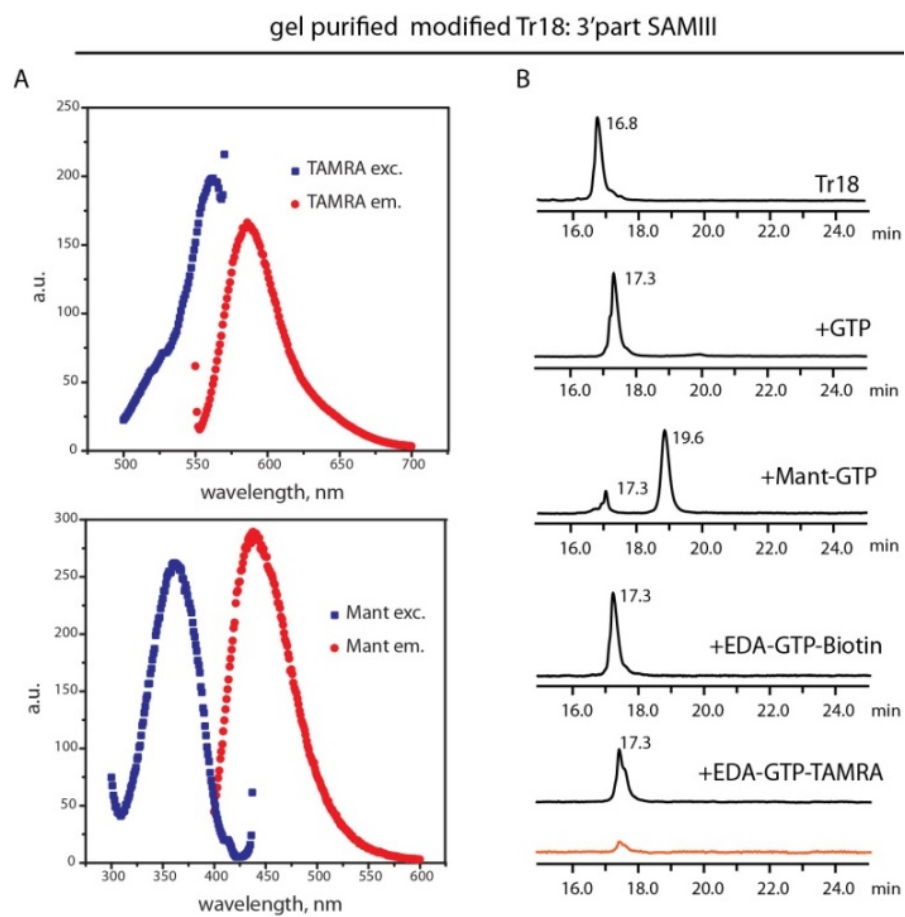

Fig 3.1-8 Confirmation of persistence of the EDA linked labels through 10DM24 reaction. A) Fluorescence spectra of 1 $\mu M$ modified Tr18 with TAMRA or Mant after gel purification of the labeled band. The measurements were done in presence of $150 \mathrm{mM} \mathrm{NaCl}, 2 \mathrm{mM} \mathrm{KCl}$ and $50 \mathrm{mM}$ HEPES pH 7.5 in $120 \mu$ volume. Excitation and emission filters were set at 355 and $445 \mathrm{~nm}$ for TAMRA (slit $5 \mathrm{~nm}$ ). Excitation and emission filters were set at 545 and $575 \mathrm{~nm}$ for Mant (Slit $5 \mathrm{~nm})$. B) HPLC chromatogram of labeled Tr18 after gel purification. HPLC gradient $30-60 \% \mathrm{~B}$ in $\mathrm{A}$ in $12 \mathrm{CV}$ at $80^{\circ} \mathrm{C}$. A: 25 mM Tris-HCl, pH 8.0, 6 M urea. B: 25 mM Tris-HCl, pH 8.0, $0.5 \mathrm{M} \mathrm{NaClO}_{4}, 6 \mathrm{M}$ urea. Black line shows $\mathrm{UV}^{260} \mathrm{~nm}$ trace. The red line in the bottom chromatogram shows detection at $545 \mathrm{~nm}$. The numbers besides the peaks, indicate retention time in min.

\subsubsection{GTP attachment to SAM III}

GTP attachment to RNA molecules was aimed to be applied for site specific labelling natural functional RNAs with modified-GTPs. SAM III riboswitch was chosen here as a model RNA for GTP labelling. The $\mathrm{S}_{\mathrm{MK}}$ box (SAM III) riboswitch regulates translation of S-adenosyl- methionine (SAM) synthetase genes in bacteria ${ }^{180}$. Upon binding to SAM, structural changes in the riboswitch lead to sequestration of the Shine-Dalgarno sequence (SD) and inhibit the translation initiation (Fig 3.1-9 A) ${ }^{181}$. The structure of aptamer domain of SAM III has been solved by X-ray crystallography ${ }^{182}$ and $\mathrm{NMR}^{181}$. We reasoned 
that installation of a FRET pair via the 10DM24 mediated attached GTP would allow study of SAM III structural changes upon binding to its ligand.

In this study a SAM III aptamer domain containing the SD sequence $\left(\mathrm{S}_{\mathrm{MK}} 51\right)^{181}$ was used as model RNA. Three point mutations were introduced to the sequence to destabilize the secondary structure of the SAM III riboswitch stem for better hybridization to 10DM24 binding arms. The SAM III riboswitch was prepared by 9DB1 3'-5' linear ligation of two RNA strands. A minimized version of 9DB1 ${ }^{139}$ was reported earlier for efficient synthesis of SAM III in our group ${ }^{79}$. The isolation yield after 9DB1 ligation was between 20-40\% (Fig 3.1-9 A and B).
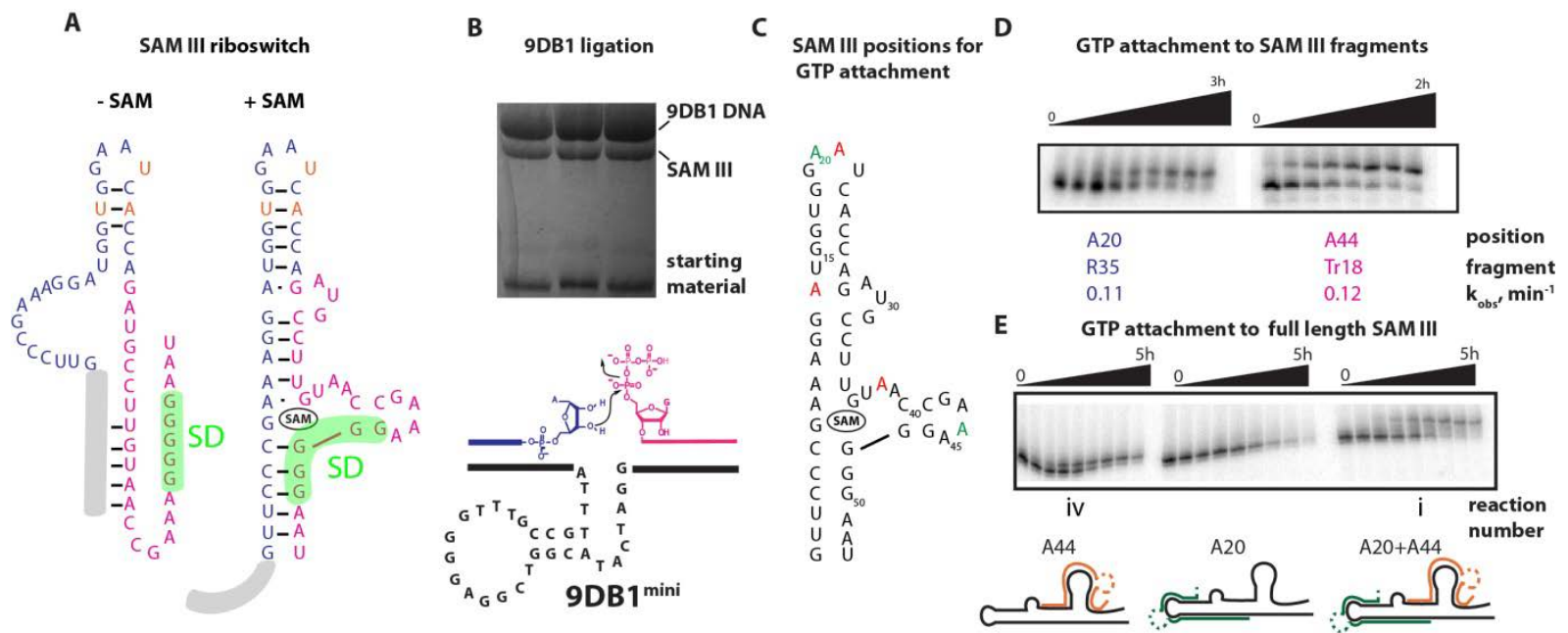

Fig 3.1-9 SAM III preparation and GTP attachment. A) SAM III sequence and its fold in presence and absence of $\mathrm{SAM}^{181}$. The sequence used in this study was based on the $\mathrm{S}_{\mathrm{MK}} 51$ reported by Wilson et al. ${ }^{181}$ The gray regions represent additional RNA sequences of $S_{M K} 59$ which are not present in our model. Three mutations were implemented to destabilized the stem loop and reduce the competing secondary structure of SAM III with deoxyribozyme binding arms. Mutation points are marked with orange letters. Full length SAM III was prepared by ligation of two shorter fragments (27 and 25 nt long). The corresponding sequences are shown with navy and magenta respectively. B) 9DB1 mediated preparative ( $2 \mathrm{nmol}$ scale) ligation of SAM III fragments. ${ }^{79}$ Minimized version of $9 \mathrm{DB} 1^{139}$ was used. C) The result of analytical scale GTP attachment to 5 adenosine candidates in SAM III sequence. Accessible positions for GTP attachment are marked green. Practically inaccessible adenosines are marked red. D) analytical GTP attachment to SAM III fragment; 5' fragment, 27nt, R35, bearing A20, navy and 3' fragment, 25 nt, Tr18, bearing corresponding sequence at SAM III for A44, magenta. Time points are 0, 1, 2, 5, 15, 30, 60, 120, 180 min for A20 and 0, 2, 5, 10, 20, 30, 60, 120 min for A44. E) GTP attachment to the full length 52nt SAM III. Condition III was applied for single and double GTP attachments at A44 and A20. Time points are 0, 2, 5, 15, $30 \mathrm{~min}, 1,2,3,5 \mathrm{~h}$. GTP concentration for single GTP attachment is $200 \mu \mathrm{M}$ and for double GTP attachment is $400 \mu \mathrm{M}$. GTP attachment to position A20 was not successful at the full length SAM III unless the deoxyribozyme for position A44 is present. Reaction numbers related to table 3.1-3 are marked in E with Roman numbers.

Initially, GTP attachment to SAM III fragments was investigated for 5 positions; A13, A20, A21 on the 5' fragment and A38 and A44 on the 3' fragment. The reactions were performed in the presence of 80 $\mathrm{mM} \mathrm{MgCl}_{2}, 100 \mu \mathrm{M} \mathrm{TbCl}_{3}$ and $200 \mu \mathrm{M} \mathrm{GTP}, \mathrm{pH} 7.5$ (condition III). Although, candidate positions for GTP attachment were selected based on the mutation studies (table 3.1-2), but successful GTP attachments were observed only for positions A20 and A44 (Fig 3.1-9 C and D). In the next step, 
accessibility of positions A20 and A44 were addressed in the full length SAM III. Full length SAM III forms higher orders of secondary structure in comparison to its fragments.

GTP attachment to position A44 was successful (Fig 3.1-7 E, and table 3.1-3 reaction iv) and reached high yield but it was slower in comparison to the corresponding position at the SAM III 3' fragment $\left(k_{\text {obs }} 0.05 \mathrm{~min}^{-1}\right.$ vs $0.12 \mathrm{~min}^{-1}$ ). GTP attachment at position 20 (Fig 3.1-7 E ) was not successful under the same condition. GTP attachment to highly structured RNAs includes two processes. First process is to resolve the RNA secondary structures by deoxyribozyme binding arms. Secondly the GTP attachment is catalyzed. As it is shown in section 3.1.3, in presence of $\mathrm{Tb}^{3+}$, 10DM24 catalysis is pretty fast in the context of GAA (sequence context for A20) and AAA (sequence context for A44). Therefore, it can be deduced that the slow reaction for position A44 and failure at position A20 is upon presence of secondary structures.

In this study, several strategies have been used to overcome the secondary structure of the SAM III riboswitch including I) simultaneous addition of both DNA enzymes II) performing the reaction at lower ionic strength or III) in presence of disruptor DNAs.

I) Simultaneous labeling

Simultaneous presence of two deoxyribozymes can improve ligation yield for highly structured RNAs. Each enzyme's binding arms disturb the secondary structure of the RNA and facilitate the hybridization of the binding arms of the other deoxyribozyme. Presence of two GTP-attaching 10DM24 deoxyribozyme results in installation of the same GTP label at the two positions which are defined by deoxyribozymes' arms. Therefore, this strategy is useful in special cases that the attachment of the same label or same functional group to the RNA is aimed. As an example attachment of two spin-labeled GTPs allows distance measurements in presence and absence of the ligand by pulsed electron-electron double resonance (PELDOR) $)^{126}$.

We attempted attachment of non-modified GTPs at two positions of the full length SAM III, A20 and A44 with GTP attaching DNA enzymes. Simultaneous presence of both enzymes lead to production of the double labeled SAM III (Fig 3.1-9 E and table 3.1-3 reaction i) but long incubation time was required. Unfortunately, long incubation time resulted in partial degradation of the SAM III RNA. At higher time points, the percentage of the labeled RNA increases in the sample but total full length RNA amount is decreased due to the site specific degradation. The "achievable yield" was calculated by the percentage of the labeled RNA at time $(\mathrm{t})$ over the total RNA present at time (0). The optimum incubation time for double labeling of SAM III with GTP attaching enzymes at condition III was $2 \mathrm{~h}$ which led to $45 \%$ maximum "achievable yield". Note that the time point for the maximum "achievable yield" is not necessarily the last time point.

II) GTP attachment in presence of reduced ionic strength 
Reduced ionic strength destabilizes RNA secondary structures and facilitates the binding of the DNA enzyme arms to the RNA substrate. On the other hand, formation of the catalytic core of the enzyme and hybridization of DNA binding arms with the RNA substrate, both require adequate ionic strength. Therefore for the best result a balanced ionic strength should be present.

Firstly, the catalytic activity of the enzyme 10DM24 for GTP attachment was investigated at low $\mathrm{Mg}^{2+}$ concentrations. At this step, the 16 nucleotide long model RNA (R16) was used as substrate in order to exclude the effect of RNA substrate secondary structures. GTP attachment to RNA model in standard condition (III) was a fast reaction as expected; $k_{\mathrm{obs}}$ : $0.9 \mathrm{~min}^{-1}$ (table 3.1-2). Concentration of $\mathrm{MgCl}_{2}$ could be reduced down to $20 \mathrm{mM}$ without severe effects on the reaction rate and yield $\left(k_{\mathrm{obs}}: 0.2 \mathrm{~min}^{-1}\right.$ in presence of $20 \mathrm{mM} \mathrm{Mn}^{2+}$, Fig 3.1-10 A)

Replacement of the $\mathrm{MgCl}_{2}$ with $20 \mathrm{mM} \mathrm{MnCl}_{2}$ resulted in a slow kinetics $\left(k_{\mathrm{obs}}: 0.001 \mathrm{~min}^{-1}\right.$ ). Addition of $10 \mu \mathrm{M} \mathrm{TbCl}_{3}$ improved the reaction rate by 10 -fold ( $\left.k_{\mathrm{obs}}: 0.01 \mathrm{~min}^{-1}\right)$. The $\mathrm{TbCl}_{3}$ concentration of 10 $\mu \mathrm{M}$ was selected because for 9F7 deoxyribozyme, the optimum $\mathrm{Tb}^{3+}$ concentration was $10 \mu \mathrm{M}$ in combination of any $\mathrm{Mn}^{2+}$ concentration. The reaction in presence of $5 \mathrm{mM} \mathrm{Mn^{2+ }}$ and $10 \mu \mathrm{M} \mathrm{Tb}^{3+}$ was even slower $\left(k_{\mathrm{obs}}: 0.001 \mathrm{~min}^{-1}\right.$, Fig 3.1-10 B).

III) GTP attachment in presence of disruptor DNAs

Disruptor DNAs were designed to disturb the secondary structure of SAM III riboswitch. D136 was designed for labeling at position 20 and was complementary to the positions 32-53 of the SAM III. D137 was designed for labeling at position A44 and was complementary to positions 1-22 of the SAM III riboswitch. Addition of 25-fold excess of the disruptor to the standard kinetic reaction (table 3.1-3 reactions vii and viii) resulted in high rate and yield. At the same time, partial degradation of SAM III was improved.
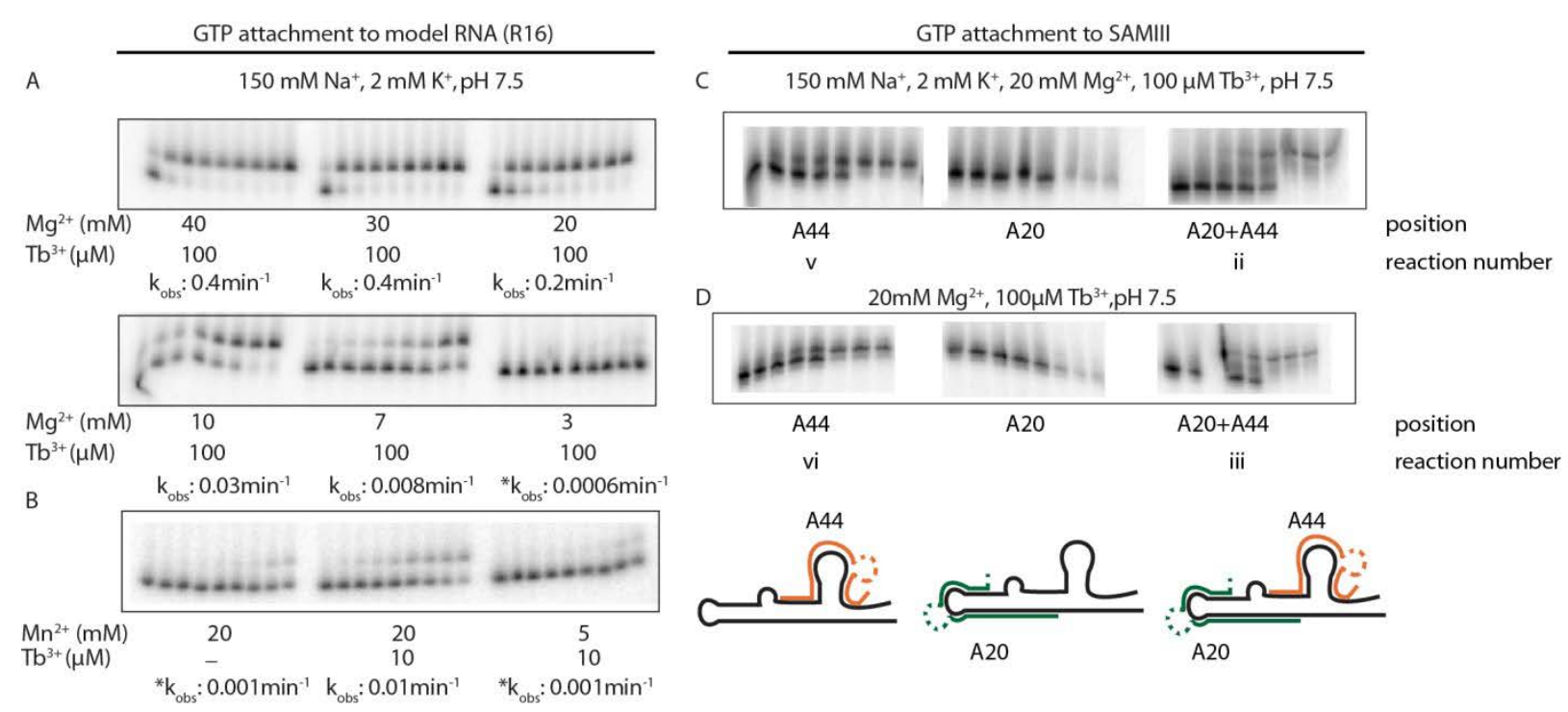
Fig 3.1-10 GTP attachment to R16 at reduced ionic strengths. A and B) investigation of the catalytic activity of the 10DM24 in presence of $\mathrm{Mn}^{2+}$ or low $\mathrm{Mg}^{2+}$ and $150 \mathrm{mM} \mathrm{NaCl}, 2 \mathrm{mM} \mathrm{KCl}$ and $50 \mathrm{mM}$ HEPES pH $7.5,200 \mu \mathrm{MTTP}$. Time points were $0,4,8,12,20,40 \mathrm{~min}, 1,2,3 \mathrm{~h}$. Where marked with star $\left(^{*}\right) k_{\mathrm{obs}}$ is defined by a linear fit. C and D) investigation of GTP attachment to full length SAM III in presence of $20 \mathrm{mM} \mathrm{MgCl}_{2}$ and $100 \mu \mathrm{M} \mathrm{TbCl}_{3}$. In D monovalent salts are absent. GTP concentration for single GTP attachment is $200 \mu \mathrm{M}$ and for double GTP attachment is $400 \mu \mathrm{M}$. The three gels in each panel are representing GTP attachment to position A44, A20 or both. Time points were $0,5,10,20 \mathrm{~min}, 1,4,6,24 \mathrm{~h}$ at $37^{\circ} \mathrm{C}$. Reaction numbers according to table 3.1-3 are marked below gels in $\mathrm{C}$ and $\mathrm{D}$.

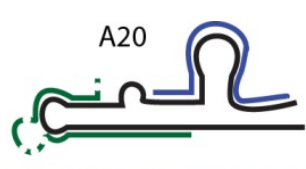

disruptor (D136) + 10DM24 for A20

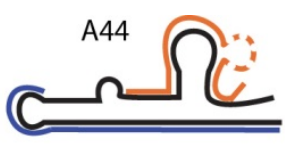

disruptor (D137) + 10DM24 for A44

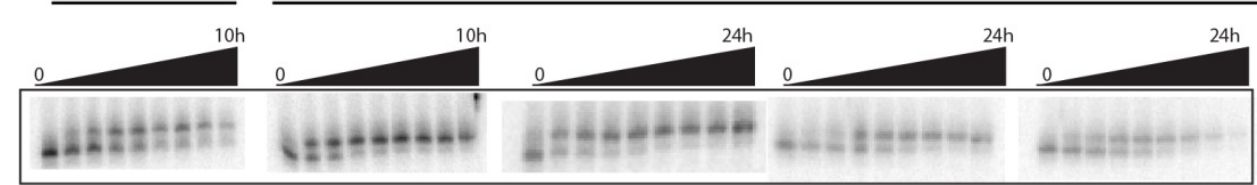
2:10:20:50
2:10:20:50
2:10:20:40
2:2:20:4
$2: 2: 2: 4$
SAM III: DNA: helper: disruptor
viii
vii
ix
$\mathrm{X}$
xi reaction number

Fig 3.1-11 Reactions for optimization of the RNA:DNA ratios for scale up. Time points for 10 hour kinetics were 0 , 5, 10, $30 \mathrm{~min}, 1,2,4,6,10 \mathrm{~h}$. Time points for 24 hour kinetics were $0,5,10,30, \min 1,2,4,8,24$ h. Reactions were performed in presence of $80 \mathrm{mM} \mathrm{MgCl}_{2}, 100 \mu \mathrm{MbCl}_{3}$ and $200 \mu \mathrm{MTP}$ at $37^{\circ} \mathrm{C}$. SAM III structure and positions at which the GTP is attached are depicted on top of the gels. The disruptors are shown blue. The reaction numbers consistent with the table 3.1-3 are mentioned below each gel.

For preparative scale reactions, equimolar ratios are preferred. Kinetic reactions with less excess of DNA enzyme and disruptor and RNA helper have been performed and their maximum "achievable yields" were calculated (table 3.1-3 reactions vii-xi). At reaction xi, which was the most similar condition to the preparative applications, incubations more than $1 \mathrm{~h}$ reduced "achievable yield". Therefore the reaction time more than $1 \mathrm{~h}$ should be avoided for practical applications

Table 3.1-3 Labeling reactions for full length SAM III in different conditions. All reactions with * were performed in the presence of $150 \mathrm{mM} \mathrm{Na}{ }^{+}, 2 \mathrm{mM} \mathrm{K}, 80 \mathrm{mM} \mathrm{Mg}^{2+} 100 \mu \mathrm{Mb}^{3+}$. The reactions with $\S$ and \# were performed in presence of $20 \mathrm{mM} \mathrm{Mg}^{2+}$ and $100 \mu \mathrm{M} \mathrm{Tb}^{3+}$ and in the absence or presence of monovalent metal ions and respectively . Gel images for reactions ( $R x$ i-xi) are marked within the chapter figures. DNA and DNA' are 10DM24 deoxyribozymes for GTP attachment at the indicated positions. Reactions were performed at $10 \mu \mathrm{l}$ at $37^{\circ} \mathrm{C}$.

\begin{tabular}{|c|c|c|c|c|c|c|}
\hline $\mathrm{Rx}$ & oligonucleotides $(\mu \mathrm{M})$ & GTP $(\mu \mathrm{M})$ & metal ions & position & max. achievable yield & $k_{\mathrm{obs}}\left(\min ^{-1}\right)$ \\
\hline $\mathrm{i}$ & \multirow{3}{*}{$\begin{array}{l}\text { SAM III: DNA: DNA': helper } \\
0.2: 1: 1: 2\end{array}$} & \multirow{3}{*}{400} & $*$ & \multirow{3}{*}{ A44-A20 } & $0.45(2 \mathrm{~h})$ & - \\
\hline ii & & & \# & & $0.31(1 \mathrm{~h})$ & - \\
\hline iii & & & $\S$ & & $0.34(1 \mathrm{~h})$ & - \\
\hline iv & \multirow{3}{*}{$\begin{array}{l}\text { SAM III: DNA: helper } \\
\qquad 0.2: 1: 2\end{array}$} & \multirow{3}{*}{200} & $*$ & \multirow{3}{*}{ A44 } & $0.52(2 \mathrm{~h})$ & 0.05 \\
\hline $\mathrm{v}$ & & & $\#$ & & $0.53(1 \mathrm{~h})$ & 0.03 \\
\hline vi & & & $\S$ & & $0.47(1 \mathrm{~h})$ & 0.05 \\
\hline vii & $\begin{array}{c}\text { SAM III: DNA: helper: disruptor } \\
0.2: 1: 2: 5\end{array}$ & 200 & $*$ & A44 & $0.93(2 \mathrm{~h})$ & 0.2 \\
\hline viii & SAM III: DNA: helper: disruptor & 200 & $*$ & A20 & $0.38(4 \mathrm{~h})$ & 0.08 \\
\hline
\end{tabular}




\begin{tabular}{|c|c|c|c|c|c|c|}
\hline & $0.2: 1: 2: 5$ & & & & & \\
\hline ix & $\begin{array}{c}\text { SAM III: DNA: helper: disruptor } \\
0.2: 1: 2: 4\end{array}$ & 200 & $*$ & A44 & $0.93(24 \mathrm{~h})$ & 0.2 \\
\hline $\mathrm{x}$ & $\begin{array}{c}\text { SAM III: DNA: helper: disruptor } \\
0.2: 0.2: 2: 0.4\end{array}$ & 200 & $*$ & A44 & 0.61 (30 min) & 0.05 \\
\hline xi & $\begin{array}{c}\text { SAM III: DNA: helper: disruptor } \\
0.2: 0.2: 0.2: 0.4\end{array}$ & 200 & $*$ & A44 & 0.48 ( 1h) & 0.07 \\
\hline
\end{tabular}

Taken together, the conditions for GTP attachment to full length SAM III was optimized. Optimized conditions for GTP and modified-GTP attachment in preparative scale for model RNAs and knowing accessible positions of full length SAM III for GTP attachment encouraged us for one step further for double labeling of full length SAM III with (modified-) NTPs in preparative scale.

\subsubsection{Site specific cleavage of SAM III}

For preparative labeling of long RNA molecules, methods need to be developed for removal of the nonlabeled- RNAs. The standard separation procedure which is based on gel retardation of GTP-attached RNAs is not useful for long RNAs. We introduced a method for purification and analysis of the efficiency of GTP attachment to large RNA molecules (section 3.1.8). The method employs the ability of RNA cleaving deoxyribozymes for site specific cleavage of substrates with free 2'-OH at the cleavage site. In this section, cleavage of SAM III riboswitch and its fragments are investigated using E5112 and 10-23 deoxyribozymes.

E5112 was originally selected in presence of $7.5 \mathrm{mM} \mathrm{MgCl}_{2}$ and $7.5 \mathrm{mM} \mathrm{MnCl} 2,50 \mathrm{mM}$ HEPES pH $7.0100 \mathrm{mM} \mathrm{KCl}$ and $400 \mathrm{mM} \mathrm{NaCl}$ and was reported to cleave $\mathrm{N} \downarrow \mathrm{A}$ linkages with the efficiency of more than $10 \%$ in $10 \mathrm{~min}^{21}$. To optimize reaction condition for the clean-up and analysis steps, nonlabeled full length SAM III was subjected to the cleavage by E5112 enzyme at A20 (D124) and A44 (D125) and by 10-23 deoxyribozyme for position A36 (D126). A variety of metal ion concentrations at pH 7.0 or 7.5 were applied. 10-23 cleavage at the junction G36-U37 was efficient on the full length SAM III i.e. more than 80\% 30 min (Fig 3.1-12 A). E5112 cleavage was not successful on the full length SAM III at any of the junctions A20-A21 and A44-A45. (Fig 3.1-12 B). The cleavage reaction was additionally performed on the SAM III 3' fragment (Tr18) which lacks the strong secondary structure of the full length. The best activity was observed at pH 9.0 in presence of $100 \mathrm{mM} \mathrm{MgCl}_{2}$. The reaction reached more than $60 \%$ in $10 \mathrm{~h}$ (Fig 3.1-12 C). 


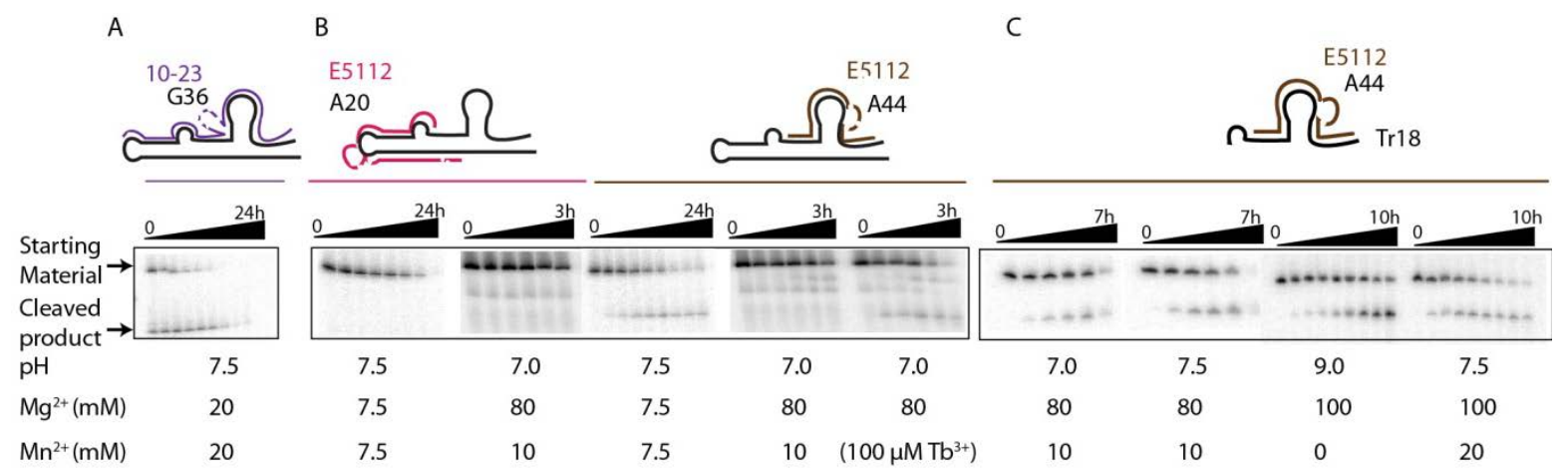

Fig 3.1-12 Cleavage of the dinucleotide junctions $\underline{A} A$ with E5112 and the dinucleotide junction GU with 10-23 enzyme. Starting materials (SM, either full length SAM III or SAM III 3' fragment; Tr18) are depicted on each gel. Times for 24 h kinetics: 0, 5, 10, $30 \mathrm{~min}$, 1, 2, 4, 8, 24 h, times for $7 \mathrm{~h}$ kinetics: 0, 5, 30, 80, $240 \mathrm{~min}$. Time points for 10 h kinetics 0, 5, 10, $30 \mathrm{~min}, 1,2,4,6,10 \mathrm{~h}$. The reactions were performed in presence $50 \mathrm{mM} \mathrm{HEPES} \mathrm{pH}$ 7.5, $150 \mathrm{mM} \mathrm{NaCl,} 2 \mathrm{mM} \mathrm{KCl}$ and indicated concentrations of $\mathrm{MnCl}_{2}$ or $\mathrm{MgCl}_{2}$ (and $\mathrm{TbCl}_{3}$ ).

Studying site specific cleavage of RNAs by 10-23 and E5112 was essential to develop the protocol for double labeling of SAM III and the removal of the non-labeled RNAs and analysis of the labeling efficiency. The protocol is described in the next section.

\subsubsection{Discussion and outlook}

The work presented in this section, is an example of transferring the basic knowledge to the application of deoxyribozymes. $\mathrm{Tb}^{3+}$ mediated rate enhancement of 3HJ forming deoxyribozymes was employed to attach GTP molecules to RNA models at specific positions. The reaction rates and the required GTP concentrations were impressively improved. Additionally, the working $\mathrm{pH}$ was reduced from 9.0 to 7.5. At kinetic scale $(0.2 \mu \mathrm{M}$ scaffold RNA, $10 \mu \mathrm{l})$, the optimum concentration for GTP was $10 \mu \mathrm{M}$ when $\mathrm{Tb}^{3+}$ was present. The reaction in presence of only 5-fold excess of GTP (1 $\left.\mu \mathrm{M}\right)$ was shown to be reasonably fast i.e. $k_{\mathrm{obs}} 0.27 \mathrm{~min}^{-1}$. For preparative scale GTP attachments, the ratio of GTP to RNA was kept 4-5 fold keeping high reaction rate, i.e. $k_{\mathrm{obs}} 1.6 \mathrm{~min}^{-1}$ (5 $\mu \mathrm{M}$ RNA and $20 \mu \mathrm{M}$ GTP in presence of $100 \mu \mathrm{M} \mathrm{Tb}^{3+}$ and $80 \mathrm{mM} \mathrm{Mg}^{2+}$ in $\left.100 \mu \mathrm{l}\right)$.

Comprehensive studies have been performed to investigate the effect of adjacent nucleotide to the branch site in presence of 4 different combinations of $\mathrm{pH}$, metal ions and helper oligonucleotides. Condition III (80 mM Mg${ }^{2+}, 100 \mu \mathrm{M} \mathrm{Tb}^{3+}$ and $200 \mu \mathrm{M}$ GTP with RNA helper at pH 7.5) was selected for further kinetic studies on any new RNA model. The mutation data also suggested that purine embedded adenosines are best positions for GTP attachment and presence of $U$ nucleotide should be avoided at the 3' neighborhood of the branch site except the 5' adjacent nucleotide is a G.

In this study, attachment of EDA-linked modified-GTPs with Cy3, TAMRA and biotin and ester linked Mant-GTP to RNA was shown to be successful in preparative scale. It was shown that the linkages survive labeling reactions (presence of $\mathrm{Tb}^{3+}$ ) and also purification steps. 
We employed our findings towards labeling of biologically related RNA molecules, SAM III and U6 snRNA. The accessible positions were identified by performing kinetic scale GTP attachment to radiolabeled fragments or full lengths RNAs. The most challenging problem for labeling of large RNA molecules was presence of secondary structures for large RNAs. Strong secondary structures compete with the hybridization of enzyme binding arms to the target sequence. We used DNA disruptors or simultaneous addition of two DNA enzymes to disturb the RNA secondary structures of SAM III. Also we increased the lengths of the DNA 3' binding arms wherever possible.

Based on our investigations, we proposed "one pot" and "sequential” protocols for double labeling of large biological RNAs. For one pot double labeling, "label-I" and "label-II" were designed to be attached to RNA via ATP and GTP respectively. Four components are then incubated with RNA substrate simultaneously: 1) ATP-attaching 10DM24 for position-I, 2) modified ATP with label-I, 3) GTP-attaching 10DM24 for position-II, 4) modified GTP with label-II. Cross-reactivity of the GTPattaching and ATP-attaching deoxyribozymes must be extensively studied for each NيN context to

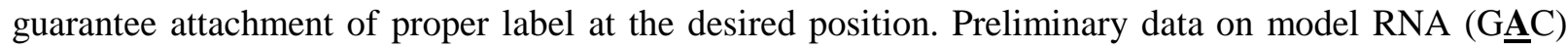
have been obtained by Lea Büttner showing low rate for mis-incorporation of GTP by ATP-attaching 10DM24 (less than 5\% in 5h) but higher rate for ATP mis-incorporation by GTP-attaching deoxyribozyme.

For sequential labeling, firstly, modified ATP with label-I is attached at position-I by ATP-attaching deoxyribozyme. Non-incorporated modified-ATPs are removed by ethanol precipitation. At this step, removal of the ATP attaching enzyme is not required (explained below). In the second step, label-II is attached via GTP using GTP-attaching deoxyribozyme which is designed for position-II.

At this step, the ATP attaching enzyme for position-I and modified-GTP with label-II are both present. The rate of mis-incorporation of GTP-label-II at position-I depends on the efficiency of ATP-label-I attachment at position-I during the first step $\left(\mathrm{P}_{\mathrm{I}}\right)$ and the efficiency of mis- incorporation of GTP-labelII at position-I during the second step $\left(\mathrm{P}_{\mathrm{II}}\right)$. Only $\left(1-\mathrm{P}_{\mathrm{I}}\right)$ fraction of the samples is available for misincorporation with the probability of $\left(\mathrm{P}_{\mathrm{II}}\right)$;

$$
P_{\text {mis-incorporation }}=\left(P_{I I}\right)\left(1-P_{I}\right)
$$

Ideally, $\mathrm{P}_{\mathrm{I}}$ is required to be $>0.8$. The yield of $80 \%$ was shown to be achievable yield for most of the NAN contexts when the reaction was performed at condition III. The mis-incorporation rate of GTP by ATP-attaching enzyme for an RNA model, studied by Lea Büttner, was only $5 \%$ in 5 h. Therefore, theoretically, under such conditions maximum $1 \%$ of the samples will be wrongly labeled with GTPlabel II at position I.

Enzymatic reactions never yield 100\% efficiency. For RNA fragments smaller than 50 nucleotides, denaturing PAGE can be applied to separate labeled and non-labeled RNAs. Labeling larger RNA 
molecules with small moieties such as modified GTPs is more challenging. Denaturing PAGE is not applicable for separation in these cases. We proposed a clean-up strategy to remove the non-labeled RNAs from the sample. After attachment of the desired labels, ligating enzyme 10DM24 are removed via denaturing PAGE and the mixture of labeled and non-labeled RNA are subjected for a cleavage reaction with RNA cleaving enzymes.

The cleaving DNA enzymes are called clean-up enzyme for this purpose and are designed to cleave at the same position in which the GTP attachment has been performed. The cleaving deoxyribozymes require the $2^{\text {‘ }}-\mathrm{OH}$ present at the cleavage site to start the nucleophilic attack to the phosphodiester backbone of the RNA. ${ }^{183}$ The RNA molecules which were ligated via $2^{`}, 5^{`}$ phosphodiester bond to the GTP-label lack the $2^{`}$-OH at the branch site and therefore at the cleavage site. Thus, the branched RNA molecules will stay intact after the clean-up step, while the non-labeled RNAs will be cleaved. The cleaved fragments of non-labeled RNA are separable from the full length RNA with the attached GTPlabel (Fig 3.1-13 A)
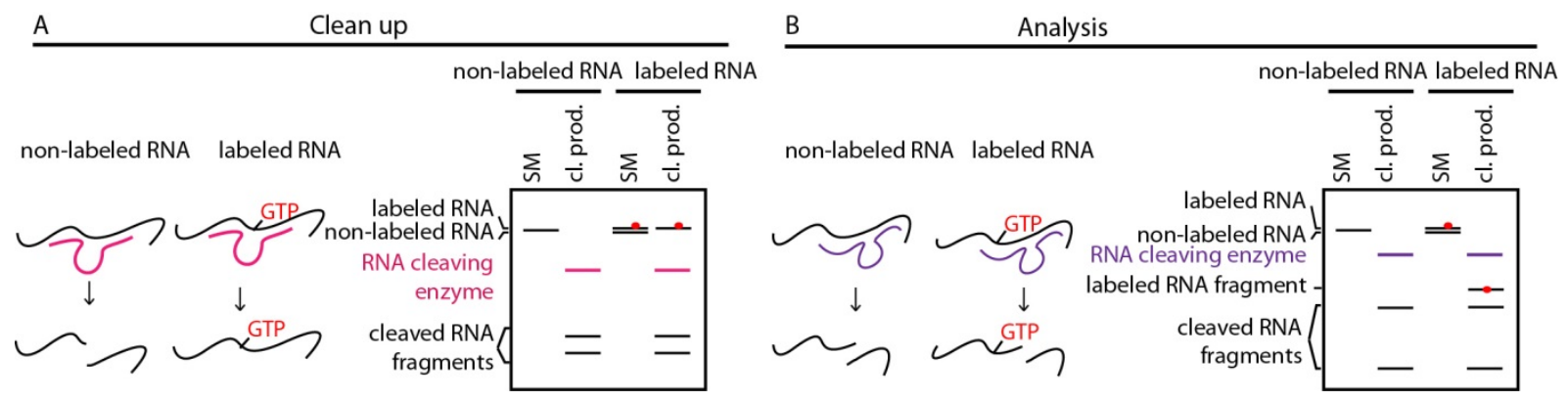

Fig 3.1-13 Clean-up and analysis strategies for long RNA labeling. A) Clean-up stratgegy for the removal of non-labeled RNA molecules. B) Analysis strategy for long RNA fragments. SM: starting material, cl. Prod.: cleaved product.

The efficiency of the clean-up deoxyribozymes and percentage of the modified RNA over non-modified RNA after the clean-up step need to be analyzed. Exploiting RNA cleaving deoxyribozymes for cleavage at distant positions from the branch site will produce smaller fragments in which presence of modified-NTPs are distinguishable. The total efficiency of ligation and clean-up steps could be analyzed by the ratio of the labeled over non-labeled short fragments. (Fig 3.1-13 B)

We have performed several experiments to find best conditions for GTP attachment to SAM III A20 and A44 in kinetic scale (0.2 $\mu \mathrm{M}$ substrate). The data are summarized in the table 3.1-3. Comparing reaction i (simultaneous double labeling of SAM III) and reactions vii and viii (attachment of a single label to SAM III in presence of disruptors) less degradation was observed for reactions vii and viii. This observation could be due to the fact that the disruptor DNAs were designed to sequester the 3' and 5' end of the SAM III and form blunt ends. 


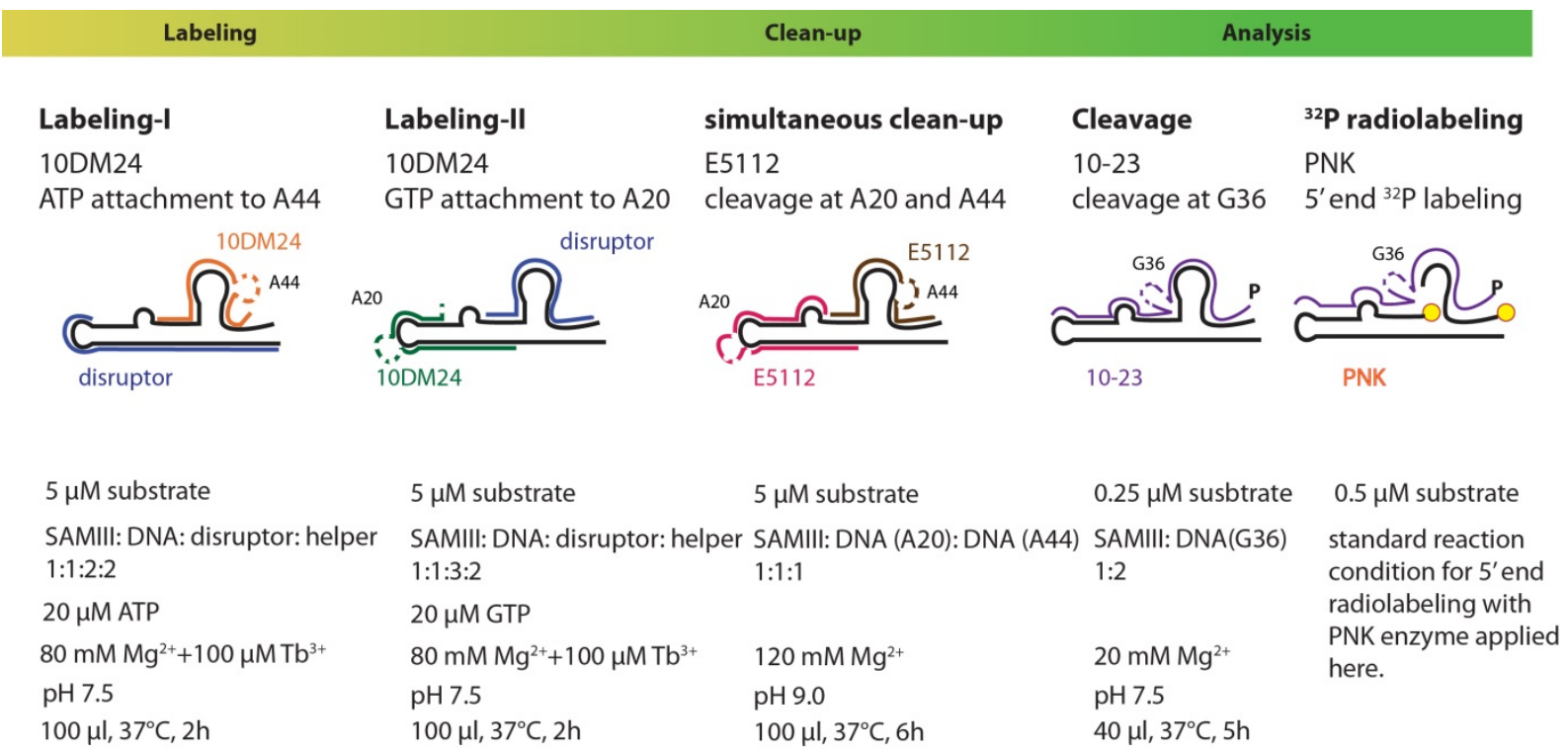

Fig 3.1-14 Schematic plan for labeling, clean up and analysis of SAM III. The branch site adenosines, length and positions of disruptor DNAs, the cleavage sites for clean-up and analysis were individually tested in previous sections. For details see the text.

Therefore, despite the advantages of the explained "sequential” double labeling strategy which does not required gel purification between the two labeling steps, in the special case of SAM III riboswitch an additional gel purification step was required for removal of the disruptors. Removal of disruptors was necessary since disruptor for labeling at position A44 sequester the binding sites for the 10DM24 for the labeling at position A20 and vice versa.

For the clean-up and analysis steps, selection of the suitable cleavage enzymes are crucial to achieve high efficiency. For SAM III, based on sequence contexts A20-A21 and A44-A45, we chose the enzyme E5112 for clean-up step. For analytical step we decided to cleave the SAM III RNA at the junction G36U37 with 10-23.

In analysis step, after analytical cleavage at distant position in respect to the labeling site, cleaved products bear a 5'-OH. For visualization reasons, all the fragments are radiolabeled with ${ }^{32} \mathrm{P}$ using PNK which is specific for 5'-OH. Fig 3.1-14 summarizes the protocol and conditions that are designed specifically for double labeling, clean-up and analysis of SAM III. Preliminary data showed successful GTP and ATP attachments at the desired positions.

The designed protocol for double labeling of U6-snRNA is shown in Fig 3.1-15. GTP attachment to short U6-snRNA fragment was shown to be successful at positions A51 and A76 with 64\% and 50\% yield in 3h (Fig 3.1-5). For full length U6-snRNA, 8-17 deoxyribozymes were designed for clean-up step for positions A51 (Cl-I) and A76 (Cl-II). For analysis, three RNA cleaving DNA enzymes were designed i.e. one 10-23 deoxyribozyme for position A35 (An-i) and two 8-17 deoxyribozymes for positions A62 (An-ii) and A97 (An-iii). 


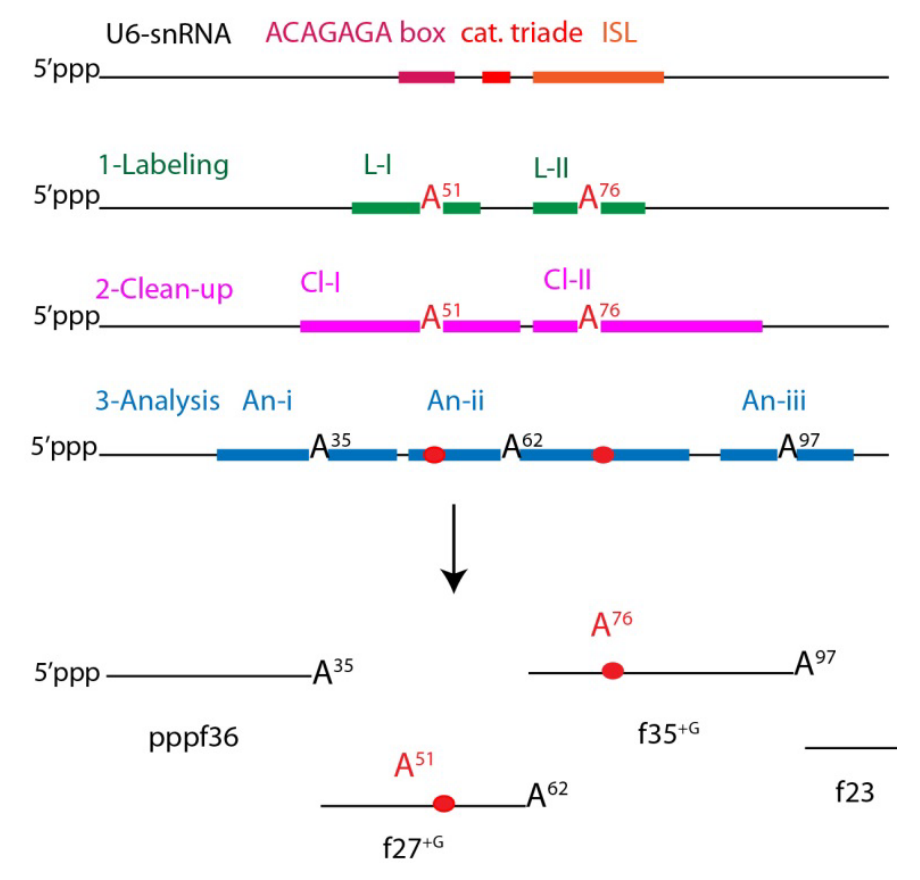

Fig 3.1-15 Schematic plan for labeling, clean up and analysis of U6-snRNA. Regions of U6 snRNA which are base paired in the 10DM24 GTP attaching enzymes or cleavage enzymes for clean-up or analytical steps are colored green, magenta and blue respectively. The adenosine branch sites and cleavage sites for cleaving enzymes are marked red and black respectively. The red circles resemble the attached GTP to the RNA.

The selection for the type of cleavage deoxyribozyme was only based on the sequence context at the cleavage site. The length of the binding arms of the DNA enzymes were designed to facilitated simultaneous GTP attachment at the labeling step, removal of non-labeled RNAs at the clean-up step and cleavage at the analysis step.

Analytical cleavages by An-1, An-2 and An-3 will result in four RNA fragments; 1) 5' fragment of U6 which is not GTP- labeled, is triphosphorylated and is 36 nucleotide long (pppf36), 2) a fragment corresponding to positions 35-62 which is supposed to be GTP-labeled at position A51, has 5'-OH and is 27 nucleotide long ( $\mathrm{f} 27^{+\mathrm{G}}$ ), 3) a fragment for positions 63-97 which is considered to be labeled at position A76, has 5 ' $-\mathrm{OH}$ and is 35 nucleotide long ( $\mathrm{f} 35^{+\mathrm{G}}$ ) and finally 4 ) the 3 ' end of the U6 which is not GTP- labeled, has 5'-OH and is 23 nucleotide long (f23).

Structural and functional studies with labeled RNAs are only valid if the label does not affect the structure and function of the target molecule. Although, the (modified-) GTPs introduced here are rather small labels their effect on the global folding of the RNA need to be investigated. Modification of RNA molecules with a fluorescent nucleoside e.g. 2-Aminopurine (2AP) at near positions to the branch point adenosine can be useful in this respect. Comparative fluorescence analysis of the non-labeled and labeled RNAs with modified-GTP, while 2AP is the fluorescent reporter of the local changes will allow detection of the structural effects of the GTP label.

To perform such studies on SAM III RNA, there are plenty of adenosines in vicinity of the promising labeling positions at which 2AP can be incorporated with little disturbances. RNA need to be 
synthesized on solid phase with SAM III sequence and bearing 2AP at position A21 or either of the positions A43 or A45, to monitor local changes at position A20 or A44 respectively. Addition of the ligand (SAM) causes structural changes on the RNA and affects the 2AP environment and therefor its emission. It is expected that the GTP labeled SAM III and non-labeled SAM III result in similar titration curves e.g. similar binding constant to SAM.

In summary, $\mathrm{Tb}^{3+}$ mediated acceleration of deoxyribozymes has been applied for post-transcriptional DNA-catalyzed labeling of RNA molecules by attachment of (modified-) GTPs. The applicability of 10DM24 deoxyribozyme for GTP attachment was improved in three aspects: 1) enhancement of reaction rate, 2) performing the reaction at $\mathrm{pH}$ nearer to neutral conditions, and 3) reduction of the GTP requirement of the enzyme. Further method developments are needed for labeling of long (>50 nt) natural RNA molecules. 


\subsection{Formation of splicing intermediates}

To expand the utility of deoxyribozymes and in particular of $2^{‘}, 5^{`}$-branch forming deoxyribozymes, in collaboration with the group of Dr. Vladimir Pena, research group macromolecular crystallography at Max Planck Institute for Biophysical Chemistry (MPI-BPC), Göttingen, Germany and the group of Professor Reinhard Lührmann, department of cellular biochemistry at MPI-BPC, we built up the basis for in vitro studies using 2',5'-branched RNAs. Studies on formation of RNA-RNA and RNA-protein spliceosomal complexes are described in this section.

The splicing of messenger RNA precursors (pre-mRNA) to mature mRNAs is a highly dynamic and flexible process. Small nuclear ribonucleoprotein particles (snRNPs) U1, U2, U4/U6, and U5 with additional proteins participate in formation of the spliceosome complex. Formation of transient inter- or intra-molecular base pairs between components of snRNPs and pre-mRNA results in several intermediate in the assembly pathway of spliceosome. The multiple transient RNA-RNA hybridizations and RNP remodelings guarantee correct recognition of 5' and 3' splice sites (SSs) and the branch point (BP), formation of catalytically active spliceosome ${ }^{184}$ and further disassembly of the complex ${ }^{185}$.

In the chemical point of view, splicing consists of two sequential phospho-transesterification reactions. In the first step the 2'-OH of the branch point (BP), located within the intron, attacks the 5' SS. In the next step, the free 3' OH at the 5' SS attacks the 3' SS and lariat RNA is removed. The core of the lariat RNA contains both 2',5' and 3'-5' phosphodiester bonds and is structurally similar to the product of the 2',5' branched-RNA forming deoxyribozymes.

Several helicases facilitate required RNA rearrangements for spliceosome activation ${ }^{186}$. For example, during spliceosome activation, Brr2 (a U5 snRNP protein), unwinds U4/U6 snRNAs. Subsequently U6 snRNA forms catalytically important U6 internal stem-loop (ISL) and base pairs with 5 '-splice site and U2 to form catalytically important ACAGAG motif and Ia and Ib base paired regions ${ }^{88}$. Crystallography data showed that the activity of Brr2 is modulated by the U5 snRNP protein, Prp8 ${ }^{187}$. Additionally, the RNase H-like domain of Prp8 was shown to be present at the catalytic core of the spliceosome ${ }^{188}$.

For yeast spliceosome, a complex of eight proteins (nineteen complex, NTC) including Prp19, was reported to be involved in stabilizing hybridizations of U5 and U6 snRNAs with pre mRNA ${ }^{189}$. In 2009, O'Keefe and coworkers have reported involvement of the Cwc2 protein, a NTC-related protein, in the binding of U6 snRNA to Prp19 ${ }^{190}$. In 2012, Lührmann and coworkers suggested that Cwc2 promotes active conformation of the spliceosome catalytic center. The hypothesis was supported by in vitro splicing, cross linking and foot printing data. It was shown that Cwc2 interacts with the U6-ISL and a region upstream of the ACAGAG motif ${ }^{191}$.

The hypothesis got strength when the crystal structure of Cwc2 reported by Pena and coworkers ${ }^{192}$. Cwc2 structure composed of an RRM domain (RNA recognition motif) and a ZnF (Zinc Finger, CCCH 
type), connector element and variable insertions. A toroidal domain (Torus domain) was shown to act as a scaffold for the ZnF and RRM domains. Finally, it was proposed that Cwc2 is a multipartite RNA binding protein. MS analysis of UV cross linked Cwc2 with U4 and U6, revealed six sites in Cwc2 which are in close contact to U6 including ZnF and RRM domains and connector element.

In collaboration with the department of cellular biochemistry and the research group of macromolecular crystallography at Max Planck Institute for Biophysical Chemistry, we aimed to investigate the complex formation between branched RNA and U2 and U6 snRNAs in presence of Cwc2 and RNase H domain of Prp8. Finding stable complexes with the branched-mRNA, snRNAs and/ or spliceosomal proteins would facilitate in vitro studies to track conformational changes during complex formation using labeled RNAs and would be a starting point for crystallization trials.

We harnessed the ability of the deoxyribozyme 6BX22 for formation of branched-RNA with sequences similar to the yeast actin core mRNA. We synthesized several branched RNAs with different sizes of the RNA strands 5' and 3' of the branch site. In addition to the branched RNA, two linear single stranded RNAs with sequence similarities to parts of U2 and pre-mRNA were included in our studies. The plasmid template for in vitro transcription of U6-snRNA was provided by the group of Professor Reinhard Lührmann, department of cellular biochemistry. The proteins for binding assays were provided by the group of Dr. Vladimir Pena, research group of macromolecular crystallography.

\subsubsection{Preparative scale synthesis of 2',5'-branched-RNA}

Preparative scale $\mathrm{Mn}^{2+}$ dependent 2', 5' branch formation have been performed using trimmed or original 6BX22. Generality of reparative scale formation of 2',5'-branched RNAs was investigated by formation of several combinations of branched products with different lengths of donor RNA and the 5' and 3' of the branch site in acceptor RNA (Fig 3.2-1 A).

The length of the acceptor RNA strand 5' of the branch point was 13-17 nucleotide long, the length of the RNA strand 3' of the branch point was between 2-17 and the length of the donor RNAs were between 11-28 nucleotide long. Synthesis of br2-br9 was a part of a collaborative project with department of Cellular Biochemistry at Max Planck Institute for Biophysical Chemistry. The effects of branched RNAs with different lengths on the nucleolytic activity of human 220K- and Prp8- RNaseHlike domains were studied by Alexey Rozov (PhD thesis, April 2011).

\subsubsection{Binding assays with 2',5'-branched RNAs}

Formation of RNA-RNA and RNA-protein complexes (consisting of 6BX22 branched RNA products) was studied by gel mobility shift assays and is described in this section. We aimed to investigate formation of three complexes consisting of branched or linear RNAs with U2 and U6 (Fig 3.2-1 C, D and E) in presence or absence of spliceosomal proteins. Fig 3.2-1 B shows the structural arrangement 
around the branch site adenosine of the yeast actin pre-mRNA with U2 and U6 snRNAs within the catalytic center of the spliceosome.

A

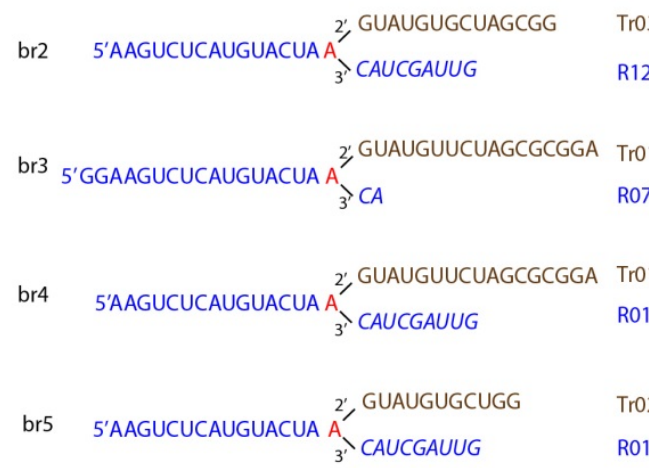

br6 5'GUCUCAUGUaCua 2', GUAUGUGCUGG Tr02

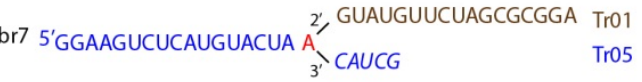

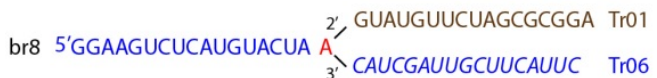

br9 5'AAGUCUCAUGUACUA 2", GUAUGUUCUAGCGCUUGCAGCAUCCCAU Tro7

B
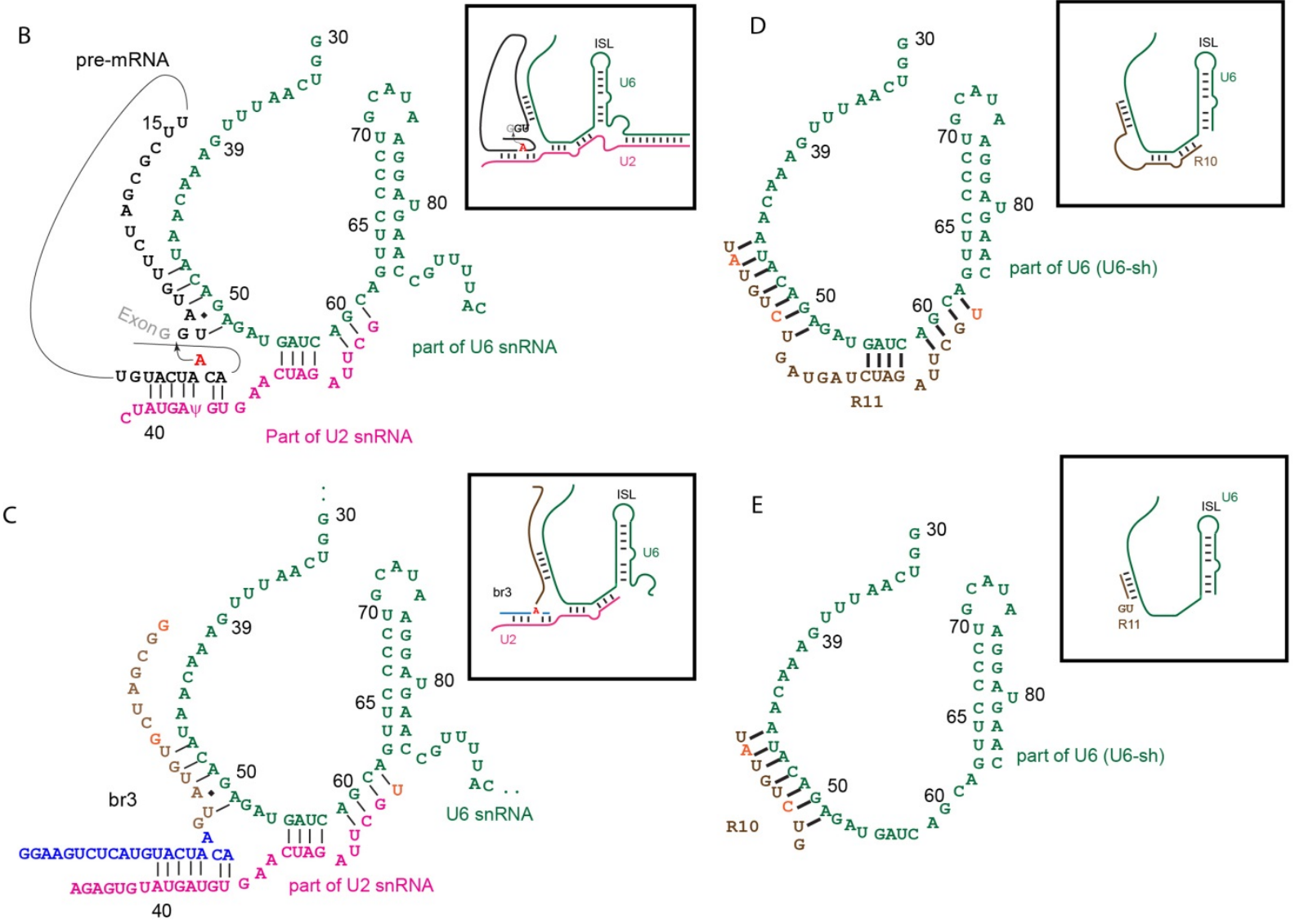

Fig 3.2-1 Branched RNAs and splicing intermediate complexes A) sequence and schematic depiction of 6BX22 branched products prepared in preparative scale. br2-br9 were part of the collaborative project with the department of Professor Reinhard Lührmann. br2 was additionally used for binding assays with spliceosomal proteins and snRNAs. B) The original sequences of yeast actin mRNA and parts of U2 and U6 snRNAs. C) The sequences of br3 in complex with part of U2 and U6-snRNAs. D and E) complexes with linear single stranded RNAs and U2 and U6-snRNAs. R11 consists of sequences similar to 5'SS and U2 snRNA. R10 resembles the 5' SS.

All the experiments were performed after annealing of RNA molecules in the presence of $150 \mathrm{mM}$ $\mathrm{NaCl}, 2 \mathrm{mM} \mathrm{KCl}$ and stated concentration of $\mathrm{MgCl}_{2}$ at $\mathrm{pH}$ 7.5. Two different experimental setups were used in the annealing step. In the first setup, serial dilutions of the stated RNA molecules $(2 \mu \mathrm{M}-1 \mathrm{nM}$ trace labeled RNA) were prepared and annealed at the indicated concentrations. The trace labeled RNAs 
are marked with a star $\left(^{*}\right)$ in the text and figures. At this set up, non-radiolabeled components were in slight excess to the labeled RNA (1.25:1).
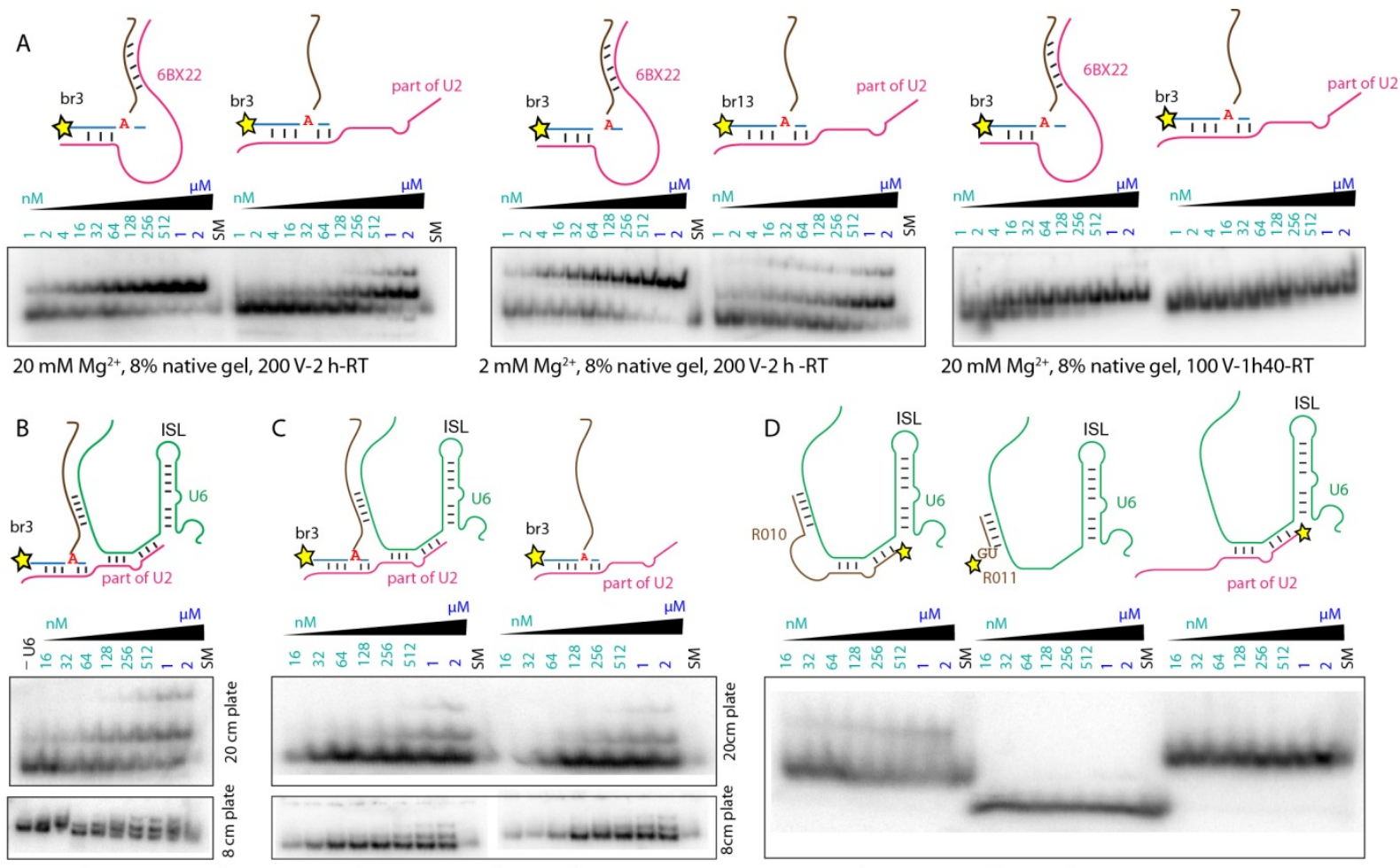

$2 \mathrm{mM} \mathrm{Mg}^{2+}, 8 \%$ native gel,

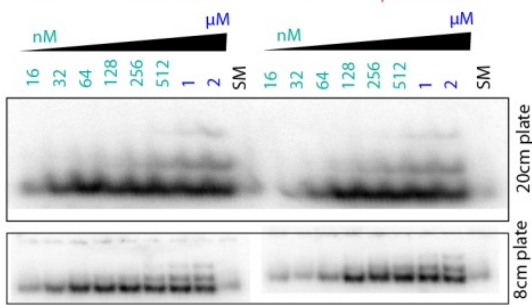

$2 \mathrm{mM} \mathrm{Mg}^{2+}, 8 \%$ native gel, $200 \mathrm{~V}-1 \mathrm{~h} 40-\mathrm{RT}$

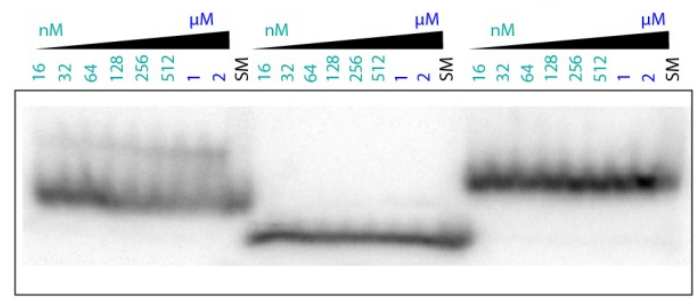

$2 \mathrm{mM} \mathrm{Mg}^{2+}, 8 \%$ native gel, $200 \mathrm{~V}-1 \mathrm{~h} 40-\mathrm{RT}$ $200 \mathrm{~V}-1 \mathrm{~h} 40-\mathrm{RT}$

Fig 3.2-2 Binding assays in the absence of proteins. A) optimization for binding assays, the effect of high and low $\mathrm{Mg}^{2+}$ concentration and voltage. The trimmed 6BX22 deoxyribozyme that has extensive binding arms for the branched product was used as a control experiment. Concentrations of the complexes are marked with green (nM) and blue ( $\mu \mathrm{M})$ colors. B) Formation of trimolecular complex by annealing in presence of all three RNAs. C) Formation of tri-molecular complex by addition of U6 RNA (or water) to the pre-annealed *br3 and U2 complex. D) Formation of bimolecular complexes with U6 snRNA.

At the first step, we optimized the conditions for the binding assays. Fig 3.2-2 A represents the effect of $\mathrm{Mg}^{2+}$ concentration and the electrophoresis voltage for a set of samples. Combination of br3 and trimmed 6BX22 was used as a positive control for binding assays. 6BX22 which was used for preparation of br3 provides two extensive arms for the branched RNA. It was observed that br3 and U2 form a complex when the complex concentration was above $60 \mathrm{nM}$. At concentrations above $0.5 \mu \mathrm{M}$ br3 and U2, a second band was visible which could be interpreted as a different conformation of br3 and U2 complex or higher order complexes. In a separate experiment, the three components of *br3, U2 and U6 snRNA were annealed together (Fig 3.2-2 B).

Similar result was observed when U6 was not present (Fig 3.2-2 A) indicating that the additional shift is not because of presence of U6. To confirm this observation, $\mathrm{U} 6$ or $\mathrm{H}_{2} \mathrm{O}$ were added to pre annealed *br3 and U2 in presence of $2 \mathrm{mM} \mathrm{Mg}^{2+}$; final ratios of U6: U2: br3 were 1:1:1 (Fig 3.2-2 C). Data were consistent with previous observations in Fig 3.2-2 B. Additionally, binding of small RNAs resembling 
U2 snRNA (R08), 5'SS (R10) or a combination of 5' SS and U2 snRNA (R11) to U6 snRNA was investigated. (Fig 3.2-2 D).

In the second setup, RNAs were annealed in relatively high concentration and diluted afterwards to reach the stated concentration. Cwc2 or RNase H domain of Prp8 proteins were added to pre-annealed br3 and U2 RNA-RNA complexes in presence or absence of part of U6-snRNA (30-83, U6-sh). To get better insights either of D121 and U2 RNA were radiolabeled in each experiment. *U2-br3 or *br3-U2 were annealed at $1 \mu \mathrm{M}$ (1:1 ratio) and diluted 100-fold. Samples were mixed with increasing concentrations of U6 and Cwc2/RNase $\mathrm{H}$ domain in presence of $\mathrm{Mg}^{2+}$ and glycerol. Final concentrations of the RNA complexes were $2 \mathrm{nM}$.

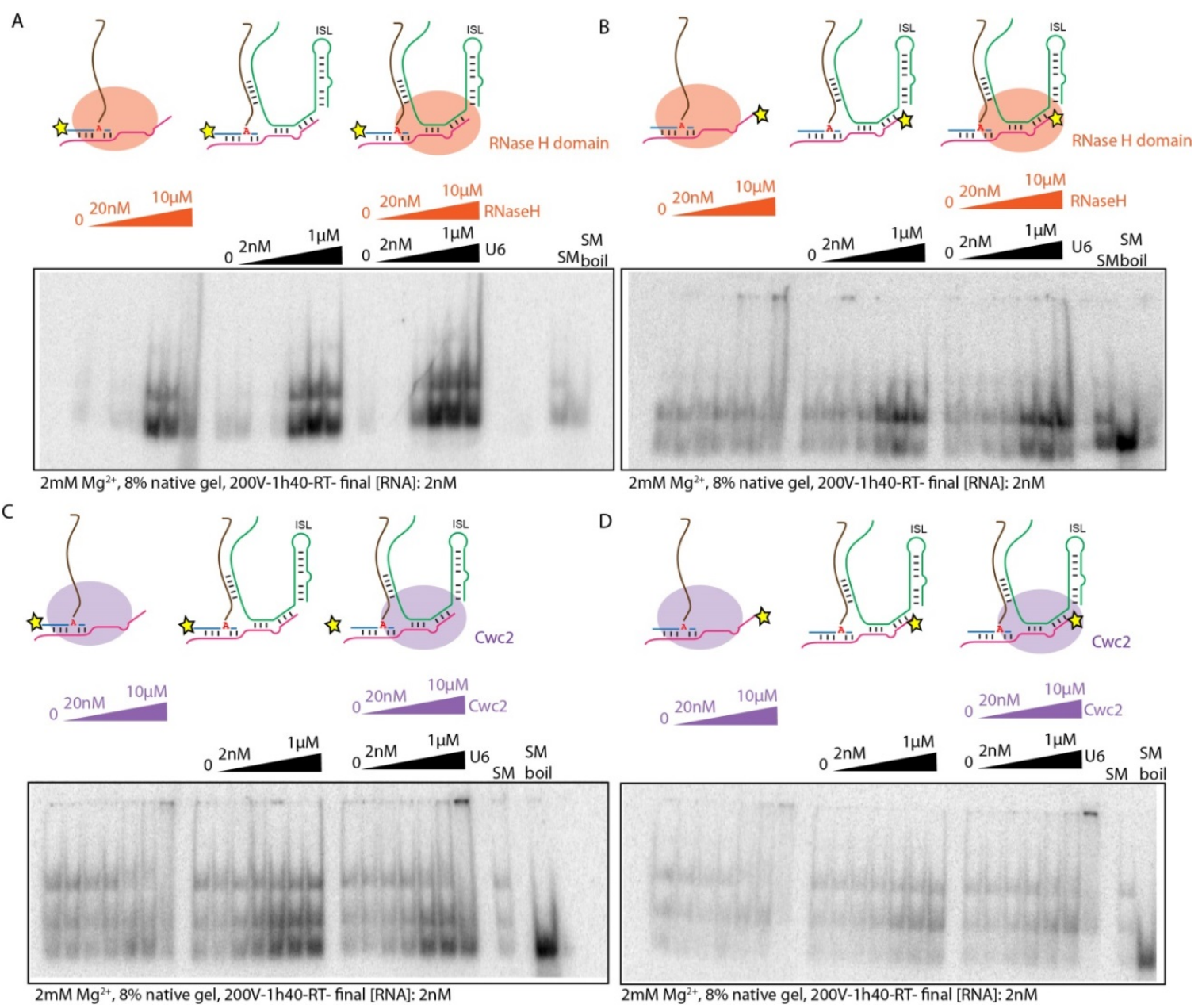

Fig 3.2-3 Titration of Cwc2 and RNase $\mathrm{H}$ domain and U6-sh snRNA to pre-annealed complexes of br3 and U2. A and B) show results for addition of $\mathrm{U} 6$ and/or RNase $\mathrm{H}$ to the mixture when the br3 or $\mathrm{U} 2$ are radiolabeled respectively. $\mathrm{C}$ and $D$ show results for addition of $\mathrm{U} 6$ and/or $\mathrm{Cwc} 2$ to the mixture when the br3 and $\mathrm{U} 2$ are radiolabeled respectively. U6 concentrations were 0, 2, 10, 20, 100, $200 \mathrm{nM}$ and $1 \mu \mathrm{M}$. Cwc2 or RNase $\mathrm{H}$ concentrations were 0, 20, 100, $200 \mathrm{nM}$ and $1,2,10 \mu M$.

The result showed addition of U6 snRNA resulted in no extra shift on the native gel even when it was provided 50-fold in excess to *U2-br3 or *br3-U2. RNase $\mathrm{H}$ domain did not cause significant binding to *U2-br3 or *br3-U2 complexes in presence or absence of U6-sh (Fig 3.2-4 A and B). Binding assays in presence of Cwc2 did not result in additional shift of the RNA-RNA complexes with the protein, but 
one of the bands in *br3-U6 complex disappeared upon presence of increasing concentrations of Cwc2. This effect was independent of presence or absence of U6 snRNA (Fig 3.2-4 C) and was not observed with br3-*U2 complex. It could be hypothesized that the Cwc2 RNA binds to one the components (e.g. non-radiolabeled U2) of one of the conformations of *br3-U2 complex, and cause dissociation of *br3.

Radiolabeled U2 was annealed with U6-sh at $4 \mu \mathrm{M}$ and diluted to final concentration of $2 \mathrm{nM}$. The samples were incubated at $5 \mathrm{mM} \mathrm{Mg}^{2+}$ at $4^{\circ} \mathrm{C}$. Increasing concentrations of Cwc2 and br3 RNA were titrated to the samples (Fig 3.2-5 A). Almost 50\% of U2 molecules formed a complex with U6-sh in the absence of protein or br3. Addition of Cwc2 protein, br3 or both did not stabilize or destabilize this complex and caused no further shift or dissociation.

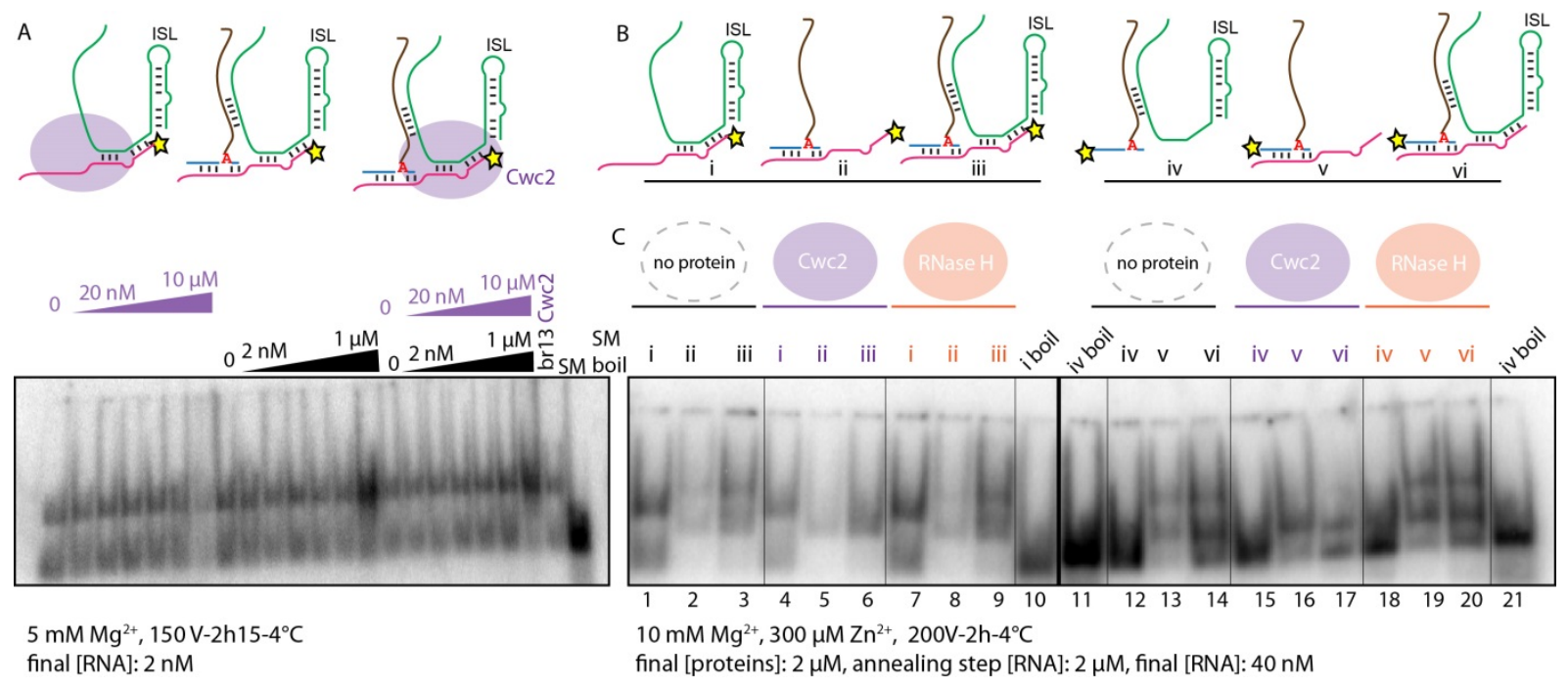

Fig 3.2-4 Comparative complex formation of Cwc2 and RNase $\mathrm{H}$ domain and U6-sh snRNA with pre-annealed complexes of br3 and U2. A) Complex formation at $5 \mathrm{mM} \mathrm{Mg}^{2+}$. br3 concentrations were 0, 2, 10, 20, 100, $200 \mathrm{nM}$ and 1 $\mu \mathrm{M}$. Cwc2 or RNase H concentrations were $0,20,100,200 \mathrm{nM}$ and 1, 2, $10 \mu \mathrm{M}$. B) RNA complexes used in gel images in C. C) comparison of complexes formed with combinations of U2, U6-sh and br3 with Cwc2 and RNase H. The RNA complexes were annealed at $2 \mu \mathrm{M}$ and diluted to the final concentration of $40 \mu \mathrm{M}$. In the lanes that protein is present the final concentration of proteins are $2 \mu \mathrm{M}$.

For a better comparison of different combinations of RNA-RNA and RNA-proteins, all six permutations of two of the three RNAs (br3, U2 and U6) with the radiolabel present at either of the br3 or U2, were annealed at $2 \mu \mathrm{M}$ and diluted to final $0.04 \mu \mathrm{M}$ and further incubated with either Cwc2 (final $2 \mu \mathrm{M}$ ), RNase $\mathrm{H}$ domain (final $2 \mu \mathrm{M}$ ) or protein dilution buffer in the presence of $10 \mathrm{mM} \mathrm{Mg}{ }^{2+}$ and $300 \mu \mathrm{M} \mathrm{Zn}^{2+}$ (Fig 3.2-5 B and C). Consistent with previous data, in the absence of proteins, more than $50 \%$ of *U2 is involved in complex formation with U6, when U2 and U6 are annealed together (i). *U2 and br3 (ii) form two complexes which stay intact when U6-sh RNA is present in addition to *U2 and br3 (iii). Addition of Cwc2 to the complex i had no effect. Cwc2 addition to complex ii decreased the ratio of the upper band in the native gel. This observation was less pronounced in the presence of RNase H domain. The same pattern was observed when the label is on the *br3 instead of *U2 (Fig 3.25 lanes 11-20). 
The results in Fig 3.2-5 B were consistent with previous data achieved in this project, in spite of slight differences in the experimental setups (compare with Fig 3.2-4 B). The samples in Fig 3.2-5 B were

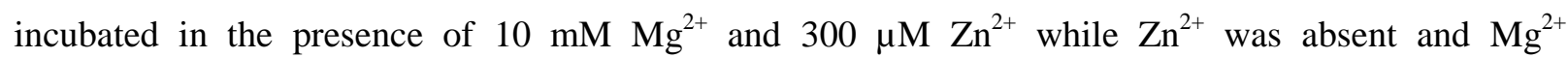
concentration was lower at all previous gels shown in this sub chapter. In summary, formation of a trimolecular format RNA complex in presence or absence of spliceosomal protein could not be achieved using the techniques and the conditions which we tested. Further investigations are required using other techniques e.g. binding assays with gel filtration.

\subsubsection{Binding assays with linear RNAs}

In comparison to the natural RNA arrangements in the catalytic core of the spliceosome, the trimolecular complex consisting of br3, U2 and U6 was more realistic. Nevertheless, besides all attempts for formation of trimolecular complex, we performed gel mobility shift assays to investigated complex formation between linear single stranded RNAs and U6 snRNA (Fig 3.2-2 D).

Here, formation of complex between R11 (resembling 5'SS and U2) and U6-sh was further investigated in the presence of 2 and $5 \mathrm{mM} \mathrm{Mg}{ }^{2+}$. The complex formation was enhanced in presence of higher $\mathrm{Mg}^{2+}$ concentration. Addition of Cwc2 did not cause further shifts in the native gels (Fig 3.2-6 A). In addition, complex formation between U6-sh snRNA (final $0.04 \mu \mathrm{M}$ ) and Cwc2 or RNase H domain (final $2 \mu \mathrm{M}$ ) was investigated in presence of $10 \mathrm{mM} \mathrm{Mg}^{2+}$. Presence of proteins only resulted in smear but not a clear band (Fig 3.2-6 B). Altogether, formation of the bimolecular complex between R11 and U6-sh was shown to be successful but addition of spliceosomal proteins did not result in any extra shift on the native gels.
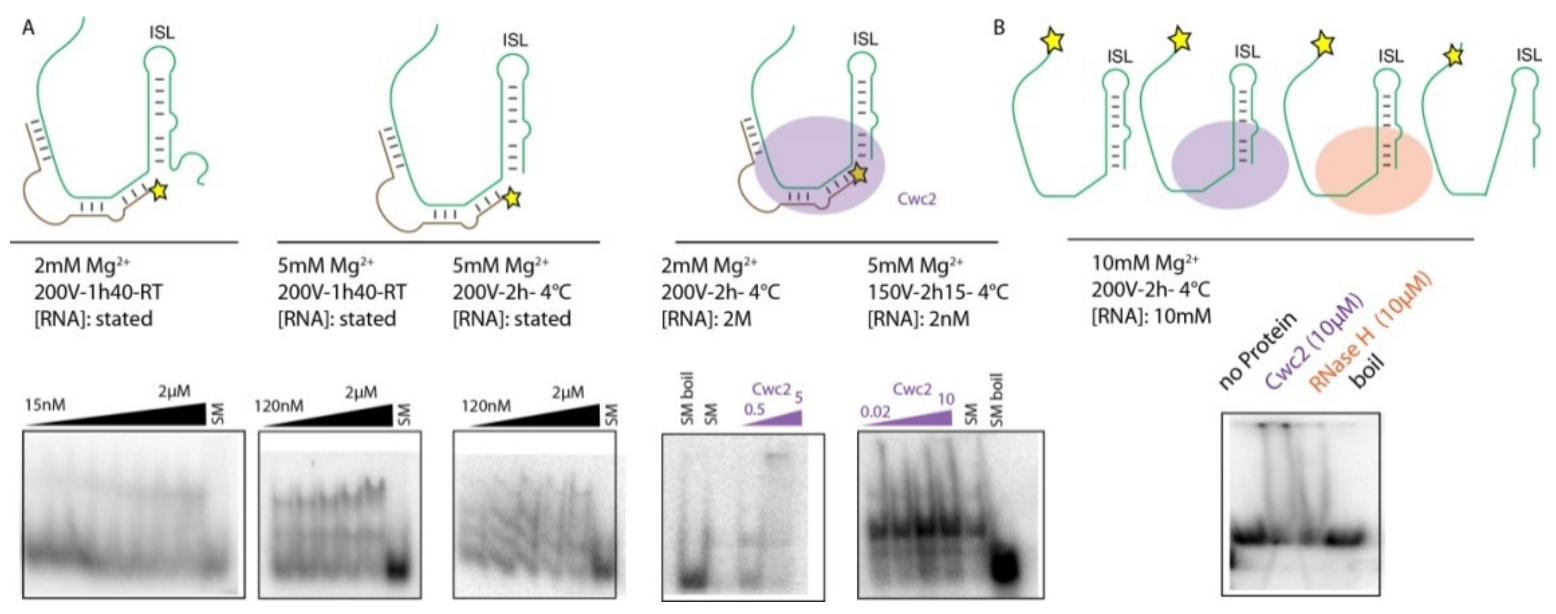

Fig 3.2-5 EMSA in the absence of branched RNA. A) Formation of bimolecular complexes with and without Cwc2. B) complex formation of U6-sh with Cwc2 and RNase $\mathrm{H}$. 


\subsubsection{Discussion and outlook}

Preparative synthesis of 2',5'-branched RNA have been achieved using 6BX22 deoxyribozymes. The 2',5'-branched RNAs are naturally found in lariat RNAs and are formed with spliceosome and group II intorns in eukaryotes and prokaryotes ${ }^{89}$. Additionally, 2',5' phosphodiester linkage is present in cyclic GMP-AMP (cGAM) which is a secondary messenger of the immune system ${ }^{193}$. An immediate application of the preparative synthesis of 2',5'-branched RNAs was to utilize them as a component for in vitro studies on spliceosomal rearrangement. Preliminary experiments that are performed and represented in this section have initiated the basis of in vitro studies with simplified lariat RNAs i.e. 2',5'-branched RNAs.

Formation of trimolecular and bimolecular RNA-RNA complexes was investigated in presence of spliceosomal proteins. The conditions used in this study did not result in formation of any trimolecular complex consisting of branched RNA (br3), and parts of U2 and U6 snRNA. Complex formation was observed U2 and br3 (forming 2 complexes) but full length U6 or U6-sh were not able to bind to the previously annealed br3 and U2 complex. Simultaneous annealing of the three components also did not improve the result. Addition of Cwc2 and RNase H domain of the Prp8 did not result in further gel retardation but in contrast presence of Cwc2 decreased the ratio of one of the complexes of U2 and br3. Hypothetically Cwc2 promotes dissociation of the two RNAs. The collected data at different conditions were consistent to each other. It was also observed that R11, consisting of sequences of 5' SS and a part of U2 snRNA is able to form a complex with U6-sh.

Electrophoresis mobility shift assay (EMSA) is a standard method for determination of the binding partners of nucleic acids and proteins. The disadvantage of the technique is that parts of multipartite complexes can be easily dissociated during gel electrophoresis and result in a false negative result ${ }^{194}$. Conversely, false positive result could be observed for binding effects that are only in non-biological context $^{195}$. The obtained data with EMSA need to be confirmed by additional biochemical investigations. Binding assays using gel filtration will support additional information about the RNARNA or RNA-protein complexes involved in splicing.

Our studies showed preliminary results on building the platform for binding assays using 2',5'-branched RNAs. With the applied experimental setup, formation of the desired complexes was not observed and additional investigations are required to optimize conditions for binding assays with 2',5'-branched RNAs. 


\subsection{Ribosomal interference by branched RNAs}

To expand the molecular and cellular basis of application of RNA ligating deoxyribozymes, we aimed to investigate the ribosomal response against presence of a branched mRNA template. We have hypothesized that a branched mRNA can stall the ribosome during peptide synthesis. For the initial step, 2',5'-branched RNAs were synthesized separately and afterwards were used for in vitro translation studies.

\subsubsection{Bacterial protein translation}

Protein synthesis consists of three steps: initiation, elongation and termination. Initiation is a crucial step in which the translation reading frame is established. Three initiation factors (IF1-IF3), fMet-tRNA ${ }^{\text {fMet }}$, $30 \mathrm{~S}$ ribosomal subunit and the mRNA, are responsible for accurate initiation and binding of initiator tRNA to the start codon in the peptidyl site (P site) of the small (30S) ribosomal subunit ${ }^{196}$. Transient $30 \mathrm{~S}$ pre initiation complex (30S PIC) is formed by assembly of initiation factors on 30S subunit and recruitment of fMet-tRNA ${ }^{\text {fMet }}$ via IF $2^{197}$. Exploiting the base complementarity between Shine-Dalgarno sequence of mRNA and the anti-Shine-Dalgarno sequence of the 3'-end of the 16S rRNA, mRNA is properly positioned in the 30S PIC.

Assembly pathway of 30S PIC is recently investigated by Rodnina and coworkers using rapid kinetic approach $^{198}$. Based on association constants and cellular concentrations of each component, it was concluded that IF3 and IF2 are the first components that bind to the 30S subunit. The assembly events are followed by binding of IF1 and fMet-tRNA ${ }^{\text {fMet } 198}$. Binding of the three initiation factors and initiator tRNA to the 30S subunit is independent from the binding of mRNA, suggesting parallel pathways of 30S PIC assembly. The recognition of the mRNA start codon by fMet-tRNA ${ }^{\text {fMet }}$ triggers conformational changes that convert the 30S PIC to a functionally competent 30S initiation complex (30S IC). 50S ribosomal subunit joins 30S IC. IF1, IF2, IF3 are released and 70S IC is formed ${ }^{198}$.

Elongation of the peptide chain consists of several steps. In the first step, a new aminoacylated tRNA is brought to the A site of the ribosome in a ternary complex. Ternary complex consists of GTP- activated elongation factor $\mathrm{Tu}$ (EF-Tu) and aminoacylated tRNA (aatRNA); EF-Tu_GTP_aatRNA. EF-Tu intrinsically is a slow GTPase. Upon codon-anticodon recognition, a conformational change induces the GTPase activity of the EF-Tu and GTP is hydrolyzed. The GDP bound EF-Tu has less affinity to the complex and is released. Release of the EF-Tu and consequently, proper positioning of aminoacylated tRNA allows peptide bond formation. The ribosomal complex is then in the pre-translocation state ${ }^{199}$. Pre- translocation complex contains peptidyl tRNA in the A site and deacylated tRNA in the P site. Elongation factor G (EF-G) is also a GTPase. EF-G initiates numerous physical rearrangements of the, rRNAs, tRNAs and ribosomal proteins that accommodate forward translocation of ribosome against mRNA by one codon. In the post-translocation complex, the deacylated tRNA is released from E site 
and peptidyl tRNA is positioned at the P site. The last step in the elongation cycle is the release of the deacylated tRNA from $\mathrm{E}$ site ${ }^{201}$.

Translation termination is dependent on the release factors. Release factors (RF1 or RF2) recognize presence of a stop codon in mRNA sequence and induce hydrolysis of the ester bond between the peptide chain and the tRNA in the P site. Peptide chain is released consequently. RF3-GTP induces replacement of RF1 and RF2 and ribosome is left with mRNA and the deacylated tRNA in the P site. Ribosomal release factor (RRF), EF-G and IF3 dissociate 50S subunit and the deacylated tRNA to prepare the $30 \mathrm{~S}$ subunit bound to mRNA for the next round of translation. ${ }^{202}$

Ribosome is a large molecular machine which is evolved to tackle secondary structure of variant mRNA templates. It is known that ribosome is capable to resolve structures such as stem loops and pseudoknots. Such structures are regulatory elements for the rate of translation and are linked to cotranslational folding and modifications of proteins ${ }^{201}$. Additionally, ribosome is able to bypass specific regions of mRNA such as slippery codons, pseudoknots or stem loops without translation. Ribosome hopping (translational bypass) in combination with programmed frame shift occurs mostly in viral mRNAs $^{203,204}$.

In collaboration with the department of Professor Marina Rodnina, department of Physical Biochemistry at Max Planck Institute for Biophysical Chemistry, we aimed to challenge the ribosome with a novel substrate i.e. a 2',5' branched mRNA. We hypothesized that ribosome should be stalled in front of the branch point but nevertheless, the possibility of translational bypass for 2',5' branched mRNAs was also investigated. We performed in vitro assays to study formation of pre-initiation complex, pretranslocation complex and proceeding elongation with branched- and linear mRNAs. “Adaptor” RNAs were ligated to a "scaffold" RNA to produce the branched mRNA. The scaffold RNA was originally a standard linear mRNA template for efficient in vitro translation and was provided by Riccardo Belardinelli, department of Physical Biochemistry.

\subsubsection{Sequence setup}

We anticipated that the location of the branch site is important for the ribosomal response to the 2',5' branched mRNA. We hypothesized that presence of the branch site in the 5' untranslated region (5' UTR) or near the initiation codon would abort formation of initiation complex. Presence of the branch site in the coding region could potentially lead to three different observations. The first possibility was the ribosome stall at the branch site. The second possibility was translation elongation at down-stream region of the mRNA (possibly with a frame shift). In the third scenario ribosome would exchange its template and the adaptor RNA (The RNA which is ligated via 2',5' phosphodiester bond to the mRNA) would be translated. 
7.5 yielded more than $70 \%$ conversion in 15 minutes. Partial degradation of the mRNA was observed at longer time points. Excluding $\mathrm{Tb}^{3+}, 40 \mathrm{mM} \mathrm{Mg}^{2+}$ at $\mathrm{pH} 9.0$ resulted in less degradation but also reduced ligation yield (Fig3.3-2 A). Adaptor ligation at position A92 was not successful even in presence of 50fold excess of disruptor DNA to the 3'end of the HemK (Fig 3.3-2 B). Preparative scale reactions have been performed to attach adaptor RNAs at position A82 in presence of $80 \mathrm{mM} \mathrm{Mg}{ }^{2+}, 100 \mu \mathrm{M} \mathrm{Tb}^{3+}$ at pH 7.5 (Fig 3.3-2 C). The branched products were separated from starting materials by denaturing PAGE. The isolated yields for branched products were 28-30\% (Fig 3.3-2 D).
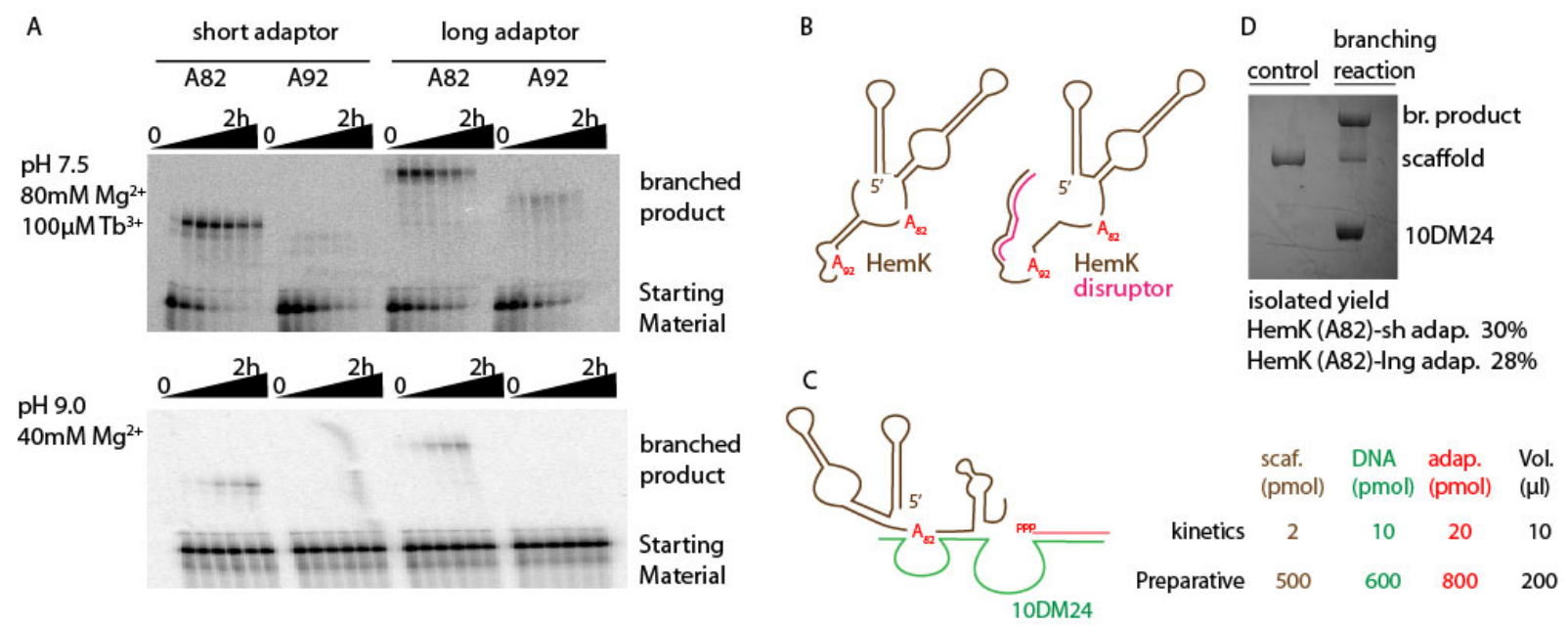

Fig 3.3-2 Ligation of adaptor RNAs to the HemK mRNA (scaffold). A) Kinetic scale branch formation was successful for position A82 of HemK. B) Schematic depiction of predicted secondary structure of Hemk mRNA in presence and absence of disrupter DNA to facilitate branch formation at position A92. C) Applying 10DM24 deoxyribozyme for site specific attachment of adaptor RNA molecules to scaffold RNA. D) Preparative branch formation at position.

\subsubsection{Formation of 70S initiation complex (70S IC)}

Formation of initiation complex with linear and branched mRNA was investigated. Ribosomal subunits $(0.1 \mu \mathrm{M}, 5 \mathrm{pmol})$ and slight excess of initiation factors IF1, IF2 and IF3 and $\left[{ }^{3} \mathrm{H}\right]$ fMet-tRNA ${ }^{\text {fMet }}$ were incubated with branched and non-branched HemK $(0-0.5 \mu \mathrm{M})$ and filtered through nitrocellulose. The amount of $\left[{ }^{3} \mathrm{H}\right]$ fMet-tRNA ${ }^{\text {fMet }}$ bound to the nitrocellulose filters was associated as the efficiency of 70S IC formation. Linear HemK mRNA formed 4.5 pmol 70S IC and provided participation of 91\% of ribosomal subunits for formation of initiation complex. Branched HemK mRNAs with short and long adaptors formed 4.0 pmol (89\%) and 4.3 pmol (87\%) 70S IC respectively. This data showed no significant difference upon presence of adaptor RNAs in formation of 70S IC (Fig 3.3-3 A).

\subsubsection{Formation of pre-translocation complex}

After successful formation of 70S IC, bacterial translation is proceeded to form pre translocation complex. We investigated the efficiency of EF-Tu mediated delivery of a radiolabeled aminoacylated tRNA to the 70S IC and attributed presence of the delivered amino acid with the formation of the pretranslocation complex. To the initiation complexes which were formed in presence of $\left[{ }^{3} \mathrm{H}\right] \mathrm{fMet}-$ tRNA $^{\text {fMet }}$, ternary complex containing EF-Tu-GTP- $\left[{ }^{14} \mathrm{C}\right]$ Glu-tRNA ${ }^{\text {Glu }}$ was added. Samples were filtered 
through nitrocellulose. The ratio of bound $\left[{ }^{3} \mathrm{H}\right]$ fMet-tRNA ${ }^{\text {fMet }}$ to $\left[{ }^{14} \mathrm{C}\right]$ Glu-tRNA ${ }^{\text {Glu }}$ reflected there is no significant difference upon presence of adaptor RNAs. In this experiment, ribosomal subunits were the limiting factors $(7.5 \mathrm{pmol})$ and RNA templates were 1.3-fold in excess to the ribosomal subunits. Incorporation of both $\left[{ }^{3} \mathrm{H}\right]$ fMet-tRNA ${ }^{\mathrm{fMet}}$ and $\left[{ }^{14} \mathrm{C}\right]$ Glu-tRNA ${ }^{\text {Glu }}$ were between 6.2-6.8 pmol for all three RNA templates. The ratio of $\left[{ }^{3} \mathrm{H}\right]$ fMet-tRNA ${ }^{\text {fMet }}$ to $\left[{ }^{14} \mathrm{C}\right]$ Glu-tRNA ${ }^{\text {Glu }}$ for non-branched HemK and branched mRNA with short and long adaptors were 1.09, 0.97 and 0.95 respectively. (Fig 3.3-3 B). The data suggested similar level of formation of pre-translocation complexes in all three samples.
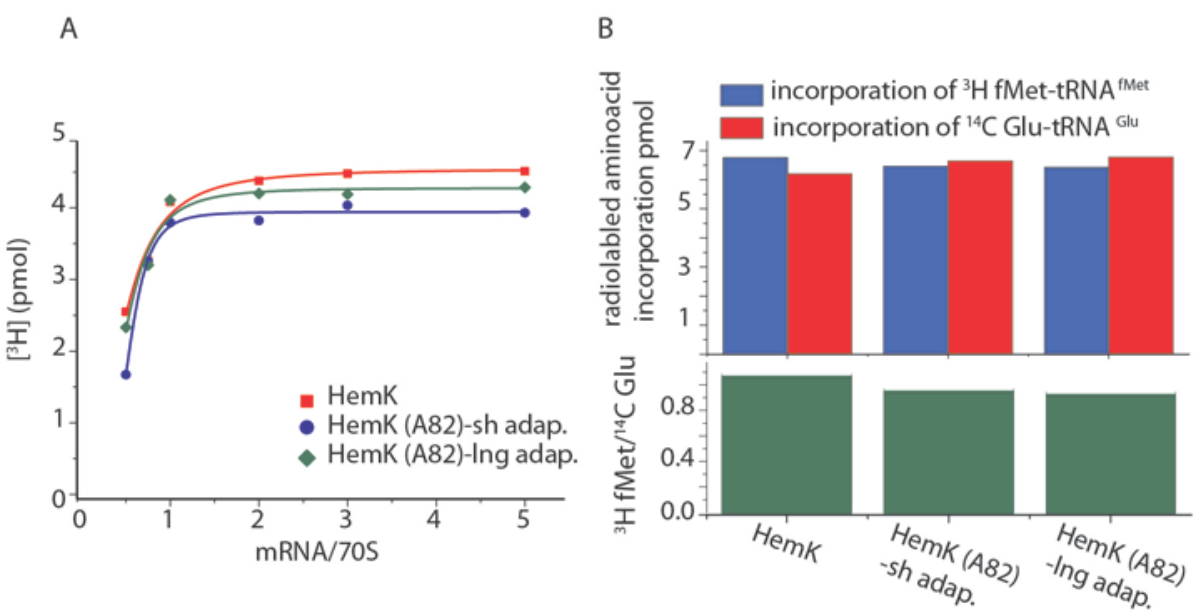

Fig 3.3-3 Formation of 70S IC and pre-translocation complex with branched and non-branched mRNAs. A) Efficiency of 70S IC formation with branched and non-branched mRNAs. No significant difference is observed. B) Efficiency of formation of pre translocation complex with branched and non-branched RNAs. There was no significant difference in the ratio of $\left[{ }^{14} \mathrm{C}\right]$ Glu (the second amino acid in the translated peptide from HemK mRNA) to $\left[{ }^{3} \mathrm{H}\right]$ fMet (initiation amino acid) for the three tested RNAs.

\subsubsection{In vitro translation}

In vitro translation of HemK mRNA, using $7.5 \mathrm{pmol}$ of ribosomal subunits as limiting factor was performed with radiolabeled $\left[{ }^{3} \mathrm{H}\right]$ Gly and $\left[{ }^{14} \mathrm{C}\right]$ Pro or $\left[{ }^{3} \mathrm{H}\right]$ Phe and $\left[{ }^{14} \mathrm{C}\right]$ Leu. The data confirmed normal translation of HemK, resulting in incorporation of 2.7 pmol $\left[{ }^{3} \mathrm{H}\right]$ Gly and 7.2 pmol of $\left[{ }^{14} \mathrm{C}\right]$ Leu. The result is consistent with two incorporation events for $\left[{ }^{14} \mathrm{C}\right]$ Leu per $\left[{ }^{3} \mathrm{H}\right]$ Gly (HemK, original frame). No glycine or proline was incorporated into the peptide translated from HemK mRNA as it was expected. Branched mRNAs with short or long adaptor RNAs did not result in any counts more than negative control (Fig 3.3-4). It was concluded that no translation occurred taking either of adaptor strands or downstream of the branch point of the scaffold strand as template (in any frame). 


\begin{tabular}{|c|c|c|c|c|c|}
\hline \multirow{3}{*}{$\begin{array}{l}\text { mRNA } \\
\text { HemK } \\
\text { (Pos. control) }\end{array}$} & \multirow{2}{*}{$\begin{array}{l}\text { radiolabeled } \\
\text { aminoacids }\end{array}$} & \multirow{2}{*}{$\frac{{ }^{3} \mathrm{H} \mathrm{dpm}}{2159}$} & \multirow{2}{*}{$\frac{{ }^{14} \mathrm{C} \mathrm{dpm}}{248}$} & \multicolumn{2}{|c|}{$\begin{array}{l}\text { incorporated } \\
\text { aminoacid (pmol) }\end{array}$} \\
\hline & & & & - & - \\
\hline & Phe/Leu & 7699 & 1507 & 2.7 & 7.2 \\
\hline HemK (A82)- & Gly / Pro & 1064 & 134 & - & - \\
\hline sh adap. & Phe/Leu & 658 & 63 & - & - \\
\hline HemK (A82)- & Gly /Pro & 2323 & 209 & - & - \\
\hline Ing adap. & Phe/Leu & 641 & 101 & - & - \\
\hline no mRNA & Gly / Pro & 2270 & 241 & - & - \\
\hline (neg. control) & Phe/Leu & 941 & 152 & - & - \\
\hline
\end{tabular}

Original frame: Met Glu Tyr Gln His Trp Leu Arg Glu Ala Phe Ser Gln Leu

Fig 3.3-4 In vitro translation of branched and non-branched RNAs. Non-branched HemK was positive control for translation of standard frame (incorporation of 1 Phe and 2 Leu per synthesized peptide). It could also be considered as the negative control for Gly and Pro incorporation. In vitro translation in presence of no mRNA was also included as the negative control. The background incorporation of each amino acid was considered separately and was deduced from the values in experimental samples.

\subsubsection{Discussion and outlook}

Ribosome is a large protein-RNA molecular machine which is capable to tackle strong secondary structures of mRNAs e.g. pseudoknots and stem loops. Here we investigated the response of the ribosome to the branched mRNA template. Our in vitro studies showed presence of the branched RNA at the $2^{\text {nd }}$ position of the $4^{\text {th }}$ codon of an mRNA does not perturb formation of 70S IC or EF-Tu mediated delivery of the aminoacylated tRNA to the second codon. In bacterial translation, after delivery of amino acids and GTP hydrolysis of the EF-Tu_GTP complex, aminoacylated tRNA is accommodated in the A site and peptide bond is formed rapidly to form the pre-translocation complex. Our method confirmed successful delivery of the second amino acid to the ribosomal complex but did not provide any data on GTP hydrolysis of EF-Tu and dipeptide formation. Additional experiments such as assays for GTPase activity of EF-Tu can support further hints to confirm formation of dipeptide.

Despite successful 70S IC formation and delivery of the second aminoacylated tRNA, in vitro translation of branched mRNAs was aborted suggesting ribosome stall behind the branch point. The branch site, A82, was very close to the start codon and only three amino acids could be incorporated before reaching the branch site i.e. to synthesize the tripeptide fMet- Glu- Tyr. Data confirmed fMet binding to the ribosomal subunits and successful Glu delivery to the A site but unfortunately no radiolabeled Tyr was available in our hand to confirm peptide formation and ribosomal translocation. Installation of branched RNA adaptors at downstream positions of the scaffold mRNA would be helpful to address the question if the branched RNA can halt the ribosome in its elongation phase. Attempts to ligate adaptor RNAs to A92 was not successful in our hand. However in the HemK mRNA sequence, there are additional candidate positions e.g. A97 and A98. Position A98 is especially beneficial since it is located at the $3^{\text {rd }}$ position of its corresponding codon. A82 was located at the $2^{\text {nd }}$ position of its codon. 
Comparing the two data sets would allow investigating the importance of position of the codon on ribosomal response.

The length of the adaptor RNA could affect final results; it is possible that the shorter adaptor has less interference with the translation. Although, we observer no difference between the two adaptor RNAs in respect to translation, it would be interesting to investigate the effect of a single GTP attachment at branch sites as adaptor. Single nucleotide GTP attachment to RNA molecules are described in the section 3.1.

Additionally, in the case of ribosomal hopping or exchange of the template strands, a short adaptor RNA might not be sufficient to fulfill the length which is required. We preformed our experiments with two adaptor lengths, 19 mer and 33 mer. No re-selection of any frames of the branched RNAs or even the scaffold RNA 3' of the branch site was observed, but nevertheless this could be a false negative result. Although one adaptor strand was longer, the extended sequence was containing only Ala, Gln and Ser in frame 1, 2 and 3 respectively (Fig 3.3-1). As we did not used labeled Ala, Gln and Ser in our experiments we have no data, in case the ribosome selects to translate from adaptor strand after 6-7 codons. Our result only confirmed that the adaptor strand until 19 nucleotide long is not taken as the template. In general, studies with a longer scaffold mRNA and adaptors and including radiolabeled amino acids, both before and after branch site, will answer the question if any frame of the scaffold or adaptor RNA is reselected by ribosomal machinery to continue peptide synthesis.

Besides answering fundamental questions about the ribosomal response to the branch site, the most promising result of our in vitro translation studies was that we were successful to abort translation of an mRNA by installation of adaptor RNAs as road blocks for ribosome. With further developments, the ribosomal interference effect of the 2',5' branching is anticipated to be applicable in the field of molecular biology as a novel RNA silencing approach.

In summary the work presented in this section shows possibility of utilizing 2',5'-branched RNAs to answer questions of molecular biology or to engineer biological systems. The application was exemplified for studying and controlling in vitro peptide synthesis. For the next steps and application of deoxyribozymes in biological systems combination of two findings would be very beneficial: 1) the peptide synthesis was aborted with a template bearing 2', 5'-linkage, and 2) the bivalent metal ion requirements of the deoxyribozyme $9 F 7$ was reduced in presence of $\mathrm{Tb}^{3+}$. New hopes are expected for the control of protein synthesis with in situ synthesis of branched RNAs catalyzed by 9F7. 


\section{General discussion}

Self-splicing group I introns and RNase P ribozyme were the first reported natural RNA catalysts. Since then several other natural ribozymes have been found most of which catalyze trans-phosphorylation reactions ${ }^{207}$. Discovery of the natural ribozymes encouraged scientists to select new and efficient artificial functional nucleic acids e.g. aptamers and synthetic ribozymes ${ }^{9}$. When the RNA catalysis was revealed in 1980s, questions raised for possibilities of DNA catalysis. Successful in vitro selection of deoxyribozymes in 1994 was an end to the discussions on the catalytic capabilities of DNA molecules. Understanding the principles of the catalytic mechanisms of deoxyribozymes is still an open fundamental question.

Currently, all discovered natural functional nucleic acids are solely RNA. Natural double stranded DNA is still only associated with the responsible molecule for genetic storage among generations. All the known deoxyribozymes are synthetic and discovered by in vitro selection. Among all deoxyribozymes, a group of DNA hydrolyzing deoxyribozymes reported by Beaker and his group are remarkable ${ }^{35}$. Breaker and his group showed that natural similar DNA sequences to these DNA hydrolyzing deoxyribozymes are also capable of catalyzing DNA hydrolysis. However, the intrinsic hydrolysis abilities of such natural sequences were weak and DNA sequences were only tested in vitro and in single stranded form in presence of $2 \mathrm{mM} \mathrm{Zn}^{2+}$.

Although Breaker and his group were cautious on their report, I would like to underline that possibility of presence of natural DNA catalysts is not completely impossible. DNA in nature is not always double stranded. Formation of Holliday junction during homologous recombination is an example of natural non-Watson-Crick DNA structure. DNA also possesses transient single stranded forms during replication and transcription. Super coiling of the circular DNAs alters their standard double helical forms. Additionally, the genome of several viruses such as bacteriophage M13 consists of only single stranded DNA molecules.

For any hypothetical natural DNA catalysts, I would assume that assistance of accessory proteins might be required for proper folding as it is observed for some natural ribozymes e.g. group II introns ${ }^{89}$. In vitro self-splicing activity of most variants of group II introns (except variant IIE and IIF) is dependent on non-physiological conditions and high salt concentrations ${ }^{208}$. The self-splicing and retrotransposition activity of the group II intron in vivo is assisted with proteins including host- and intron-encoded proteins such as endonucleases and a reverse transciptase ${ }^{209}$.

In addition to fundamental interests to understand the mechanism of DNA catalysis, functional characterization of deoxyribozymes will boost the applicability of these catalysts. The presented study in this thesis started with fundamental questions and was spun toward application when impressive $\mathrm{Tb}^{3+}$ 
mediated acceleration of RNA ligating deoxyribozymes was found. The discussion is presented in two parts for characterization and application of deoxyribozymes.

\subsection{Functional characterization of deoxyribozymes}

(Deoxy)ribozymes depend strongly on metal ions for catalysis and folding. In the case of ribozymes, crystal and solution NMR structures and vast biochemical investigations allowed scientist to elucidate their potential mechanisms and metal ion binding sites. For example, mechanisms are proposed for nucleolytic ribozymes such as hammerhead or HDV ribozyme. The mechanisms involve activation of the 2'-OH at the cleavage site for the nucleophilic attack to the scissile phosphate group of the RNA backbone $^{7}$. In contrast, the techniques and data for DNA catalysts are very restricted. No crystal structure of any active form of any deoxyribozyme is present ${ }^{94}$ and methods on functional characterization of functional DNAs were limited at the beginning of this project.

\subsubsection{Combinatorial mutation interference analysis revealed necessary nucleotides for catalysis}

At the time of starting this $\mathrm{PhD}$ project, understandings about the three dimensional folding of deoxyribozymes was little and only few reports were available on the metal ion binding sites of DNAcatalysts $^{135}$. In the case of ligating deoxyribozymes, the available scientific knowledge was only characterization of their substrate requirements. To investigate nucleotides involved in formation of the catalytic center of deoxyribozymes, conventional mutagenesis methods were possible. However, synthesis and characterization of single point mutants are laborious (3 mutations per position and for example 40 positions in catalytic core of the deoxyribozymes) and development of new methods was necessary.

The work presented in this thesis started with the development of a combinatorial approach for simultaneous investigation of all three possible mutations of all nucleotides of a catalytic DNA at a given condition. Combinatorial mutation interference analysis (CoMA) ${ }^{139}$ revealed necessary nucleotides of several deoxyribozymes (6BX22, 9F7 and 9F13 are reported in this thesis).

CoMA allowed minimization of the deoxyribozymes by two third of their original length without affecting the catalytic efficiency of the deoxyribozymes. In the case of 6BX22, the deoxyribozyme was reported for catalysis of formation of lariat RNAs from biologically related RNA sequences. Lariat formation ability of 6BX22 was kept intact after minimization.

CoMA was proven to be a reliable and sensitive method to compare the nucleobase requirement of functional DNAs and in particular deoxyribozymes. The second step of CoMA at which the catalytic reaction of the deoxyribozyme is taking place is a critical step. Performing the catalytic reaction of the deoxyribozyme at different conditions such as different time points at this step and comparison of the data allowed us to distinguish slow mutants from inactive mutants (studied on 6BX22). Performing the 
catalytic reaction of the deoxyribozymes in presence of different metal ions reflected the potential candidates for formation of the metal ion binding sites (studied on 9F7). Further biochemical and biophysical studies such as spectroscopic investigations of active and inactive mutants of 9F7 i.e. sensitized luminescence of $\mathrm{Tb}^{3+}$, DMS and DNase probing assays and phosphorothioate rescue experiments supported CoMA data on 9F7.

\subsubsection{Nucleotide analogue interference mapping of DNA provided data on functional group demands of catalytic DNAs}

In addition to CoMA, Nucleotide analogue interference mapping of DNA (dNAIM) ${ }^{140}$ was applied to characterize the minimized version of 6BX22. dNAIM is a complementary method to CoMA and elucidates the functional groups demands of the necessary nucleotides of a deoxyribozyme. dNAIM also benefits from the combinatorial approach which was introduced by CoMA. More recently combinatorial nucleoside-deletion-scanning mutagenesis (NDS) is also reported by our group ${ }^{210}$. NDS employs one library of deletion mutants and allows fast detection of the nucleotides which are not involved in formation of the catalytic core of the deoxyribozyme.

In the case of $6 \mathrm{BX} 22$, the minimized version of the deoxyribozyme contained a G-rich sequence, suggesting presence of a G-quadruplex in its structure. dNAIM provided data to disprove possibility of presence of a G-quadruplex in 6BX22 structure. Other biophysical and biochemical data from CD spectroscopy, porphyrine TMPyP4 binding assays and kinetic analyses in presence of different metal ions were in agreement with the dNAIM data and rejection of the hypothesis of presence of a Gquadruplex in 6BX22 catalytic core.

\subsubsection{Lanthanides have been useful for fundamental studies on deoxyribozymes}

We utilized lanthanide ions and in particular $\mathrm{Tb}^{3+}$ as a probe for functional analysis of deoxyribozymes. Lanthanide ions have the ability to replace $\mathrm{Mg}^{2+}$ ions in their specific binding sites and have been used to study $\mathrm{Mg}^{2+}$ binding sites with footprinting assays and with spectroscopic methods. Sensitized luminescence of $\mathrm{Tb}^{3+}$ was reported to study metal ion (in particular $\mathrm{Mg}^{2+}$ ) interaction with ribozymes ${ }^{126}$ and G-quadruplex DNAs ${ }^{124,123}$. Lanthanides have rich spectroscopic properties when they are site bound to the DNA or RNA nucleobases ${ }^{115}$. Importantly, inner sphere coordination of lanthanide ions is required for detectable luminescence. To study metal ion interactions with the 2',5'-branched RNA forming deoxyribozymes, binding of $\mathrm{Tb}^{3+}$ to the deoxyribozymes must have been proven.

Impressively and beneficially, micromolar concentrations of lanthanides (especially studied with $\mathrm{Tb}^{3+}$ ) accelerated most of the 2',5'-branched RNA forming deoxyribozymes in this study i.e. 9F7, 9F13, 7S11 and 10DM24 when millimolar concentrations of bivalent metal ions were present. In contrast, 6BX22 deoxyribozyme was inhibited in presence of $\mathrm{Tb}^{3+}$ at concentrations above $50 \mu \mathrm{M}$. $\mathrm{Tb}^{3+}$ mediated inhibition of 6BX22 was reversible and addition of $\mathrm{Mn}^{2+}$ relieved the inhibition. 
An optimal concentration of $\mathrm{Tb}^{3+}$ was required for maximum acceleration of the deoxyribozymes. For $\mathrm{Mg}^{2+}$ dependent reactions of $9 \mathrm{~F} 7$, the $\mathrm{Tb}^{3+}$ optimal concentration was $100 \mu \mathrm{M}$. Higher $\mathrm{Tb}^{3+}$ concentrations caused fast reaction of only a fraction of the available RNA substrate, resulting in reduced amplitude with retained high rate constant. Addition of $100 \mu \mathrm{M} \mathrm{Tb}{ }^{3+}$ to $\mathrm{Mg}^{2+}$ dependent reactions of 9F7 accelerated the reaction rate up to 10000 fold $\left(k_{\text {obs }} 7 \times 10^{-6}\right.$ vs 0.08 for $7 \mathrm{mM} \mathrm{Mg}^{2+}$ vs 7 $\left.\mathrm{mM} \mathrm{Mg}{ }^{2+}+100 \mu \mathrm{M} \mathrm{Tb}^{3+}\right)$. Impressively, the reaction rate in presence of $7 \mathrm{mM} \mathrm{Mg}^{2+}$ and $100 \mu \mathrm{M} \mathrm{Tb}^{3+}$ was higher than the reaction rate in presence of $400 \mathrm{mM} \mathrm{Mg}^{2+}$ and in the absence of $\mathrm{Tb}^{3+}$. The optimal concentration of 9F7 was almost the same for other lanthanide ions i.e. $\mathrm{La}^{3+}, \mathrm{Ce}^{3+}, \mathrm{Eu}^{3+}, \mathrm{Gd}^{3+}$ and $\mathrm{Yb}^{3+}$ but reduced to $10 \mu \mathrm{M}$ for $\mathrm{Mn}^{2+}$ dependent reactions of $9 \mathrm{~F} 7$.

Presence of optimal concentration of lanthanides reduced the bivalent metal ion requirements of the deoxyribozyme 9F7. The apparent binding constant for $\mathrm{Mg}^{2+}$ and $\mathrm{Mn}^{2+}$ were reduced ca. 4-fold. Reduction of the required $\mathrm{Mg}^{2+}$ concentration to near cytosolic concentrations will open opportunities for in vivo application of such deoxyribozymes especially as a tool in cellular and molecular biology.

Based on these biochemical studies, presence of two types of binding sites was proposed for 9F7. One group of metal ion binding sites was proposed to be important for catalysis. The other group was assumed to only play a role for proper folding and being peripheral. The catalytically important metal ion binding sites of 9F7 must be occupied with bivalent metal ions for catalysis. The peripheral binding sites cause acceleration of the deoxyribozyme when they are occupied with $\mathrm{Ln}^{3+}$. This hypothesis was mainly based on two observations. Firstly, 9F7 deoxyribozyme was confirmed to be absolutely dependent on presence of bivalent metal ions. In the absence of $\mathrm{M}^{2+}$ and presence of a wide concentration range of $\mathrm{Tb}^{3+}$, no product formation was observed up to $48 \mathrm{~h}$. Secondly, the biphasic titration curve of 9F7 activity with increasing concentrations of lanthanides, suggested both synergistic (below optimal concentration) and antagonistic (above optimal concentration) effects of $\mathrm{Ln}^{3+}$ and $\mathrm{M}^{2+}$ on 9F7 activity.

\section{2 $\mathrm{Tb}^{3+}$ mediated acceleration of RNA-ligating deoxyribozymes enhanced deoxyribozymes' application}

At the time of the start of this project, it was expected that functional and chemical characterization of deoxyribozymes would lead to improved applicability of such enzymes. Gratefully, the presented work in this thesis is an example of such direct impact of fundamental studies on the application of a system. $\mathrm{Tb}^{3+}$ mediated acceleration of branched RNA formation shifted reaction rates into a region that makes DNA enzymes practical for applications in RNA engineering and molecular biology.

An immediate in vitro application of $\mathrm{Tb}^{3+}$ mediated acceleration was in the direction of site specific labeling of RNA. Biochemical and biophysical studies on natural RNAs such as riboswitches require site specific functionalization of the RNA molecules to install probes such as florescent dyes. Current 
methods are based on solid phase synthesis of functionalized or labeled RNA. RNA molecules smaller than 50 nt are reliably synthesized by solid phase synthesis. For longer RNAs, smaller labeled RNA fragments are ligated with T4 RNA ligase. Thereby, the current protocol is time and material consuming and there are rooms for improvements. Development of a fast and efficient method for site specific labeling of RNA using deoxyribozymes is reported in section 3.1 of this thesis. Deoxyribozyme mediated post synthetic labeling of RNAs is independent from the length of the RNA molecules and therefore is advantageous over the current protocols.

\subsubsection{Site-specific labeling of RNA with modified GTPs was impressively efficient in presence of $\mathrm{Tb}^{3+}$}

Site-specific attachment of a single GTP to a model RNA was successfully reported using 10DM24 ${ }^{179}$. Studies in our lab (currently unpublished), showed that 2'-modifications of GTP are allowed for the GTP attachment reaction. The reaction demanded high pH and high concentration of GTP $\left(k_{\mathrm{obs}} 0.1 \mathrm{~min}^{-1}\right.$ in presence of $3 \mathrm{mM}$ GTP, $100 \mathrm{mM} \mathrm{Mg}{ }^{2+}$ at $\mathrm{pH}$ 9.0). Addition of $100 \mu \mathrm{M} \mathrm{Tb}^{3+}$ to the reaction in presence of $80 \mathrm{mM} \mathrm{Mg}^{2+}$ reduced the GTP requirement to an optimal concentration of only $10 \mu \mathrm{M}$. The $\mathrm{pH}$ of the reaction was also reduced to more neutral $\mathrm{pH}$. The reaction rate of GTP attachment in presence of $10 \mu \mathrm{M}$ GTP, $80 \mathrm{mM} \mathrm{Mg}^{2+}$ plus $100 \mu \mathrm{M} \mathrm{Tb}^{3+}$ at $\mathrm{pH} 7.5$ was above $1.0 \mathrm{~min}^{-1}$ which means that the reaction was completed within the first minute. In the absence of $\mathrm{Tb}^{3+}$, at such low concentration of GTP, formation of the product was undetectable.

Careful analysis for the preparative GTP attachment to a model RNA revealed that only 4-5 fold excess of GTP to RNA substrate is sufficient to reach a $k_{\text {obs }}$ above $0.1 \mathrm{~min}^{-1}$ when the reaction is performed at $80 \mathrm{mM} \mathrm{Mg}^{2+}+100 \mu \mathrm{M} \mathrm{Tb}^{3+}$ at $\mathrm{pH} 7.5$ and the $\mathrm{Tb}^{3+}$ : DNA ratio is above 10 . To study the generality of the method, comprehensive studies were performed to investigate the effect of point mutations adjacent to the adenosine at the GTP attachment site. The mutation studies revealed that purine embedded adenosines are the best sites for GTP attachment. The data was cross validated on several other model RNAs. Furthermore, it was shown that 2'-modified GTPs with EDA- or ester- linked fluorescent dyes were efficiently attached to the model RNA and more importantly, the EDA and ester linkages resisted the reaction condition.

Preparative labeling of large natural RNA molecules demands further method developments to tackle two main challenges. First of all, the secondary structures of large natural RNAs compete with the hybridization of the DNA binding arms to the RNA substrate. Performing the reactions in presence of disruptor DNAs might be helpful in this respect. The second main challenge is the removal of the unreacted RNAs. For the RNA molecules smaller than 50 nt separation of GTP attached RNAs from intact RNAs is possible by denaturing gel electrophoresis. For longer RNA molecules, a clean-up strategy was proposed in this thesis for removal of the unreacted RNA molecules. The proposed strategy is based on the DNA-catalyzed site specific cleavage of the unreacted RNA molecules for which the 2'$\mathrm{OH}$ of the GTP attachment site is available. Formation of the 2',5' phosphodiester bond upon 
successful GTP attachment at the branch site adenosine excludes possibility of the cleavage for the reacted RNA molecules. Efficiency of this strategy must be further investigated practically.

\subsubsection{Preparative synthesis of 2',5'-branched RNA was shown to be useful in molecular biology}

Besides attempts for applying deoxyribozymes for RNA labeling, synthesized 2',5'-branched RNA have been used to study bacterial peptide translation in vitro. Presence of an adaptor RNA to an mRNA (scaffold RNA) aborted peptide synthesis as it was expected. Although further carful inspections are required to exclude the possibility of ribosomal hoping, current data suggests that installation of a branched RNA can halt ribosomal translocation and abort protein synthesis. Finally, formation of branched RNAs with RNA fragments with naturally related sequences allowed in vitro binding assays with RNA and protein components of yeast spliceosome such as U2 and U6 snRNAs and Cwc2 and RNase H domain of Prp8.

In summary, the reported work in this thesis provided fundamental insights in the world of DNAcatalysts with development of combinatorial mutation interference analysis (CoMA) and by employing lanthanide ions as cofactors. Lanthanides and in particular $\mathrm{Tb}^{3+}$ mediated acceleration of 2',5'-branched RNA forming deoxyribozymes allowed mechanistic characterization of deoxyribozymes in respect to their metal ion binding sites. Impressive rate acceleration upon presence of $\mathrm{Tb}^{3+}$ was employed for sitespecific labeling of RNA molecules with beneficial reduced requirements on the valuable modified GTPs. Further development of the methods is aimed for preparative labeling of large natural functional RNAs such as riboswitches, snRNAs and mRNAs. 


\section{Material and methods}

\subsection{RNA and DNA preparation}

Non-modified DNAs have been purchased from Sigma Aldrich. RNAs and modified DNAs for CoMA and dNAIM were synthesized by solid phase synthesis. RNAs with 5' triphosphate were transcribed using T7 RNA polymerase. RNA and DNAs were purified by denaturing PAGE and the synthesis quality was controlled by anion exchange HPLC. Plasmid template for U6 snRNA transcription was provided by department of Professor Reinhard Lührmann. The transcribed mRNA for ribosomal interference studies was provided by the department of Professor Marina Rodnina.

\subsection{Solid phase synthesis}

\subsubsection{Chemicals and building blocks for solid phase synthesis}

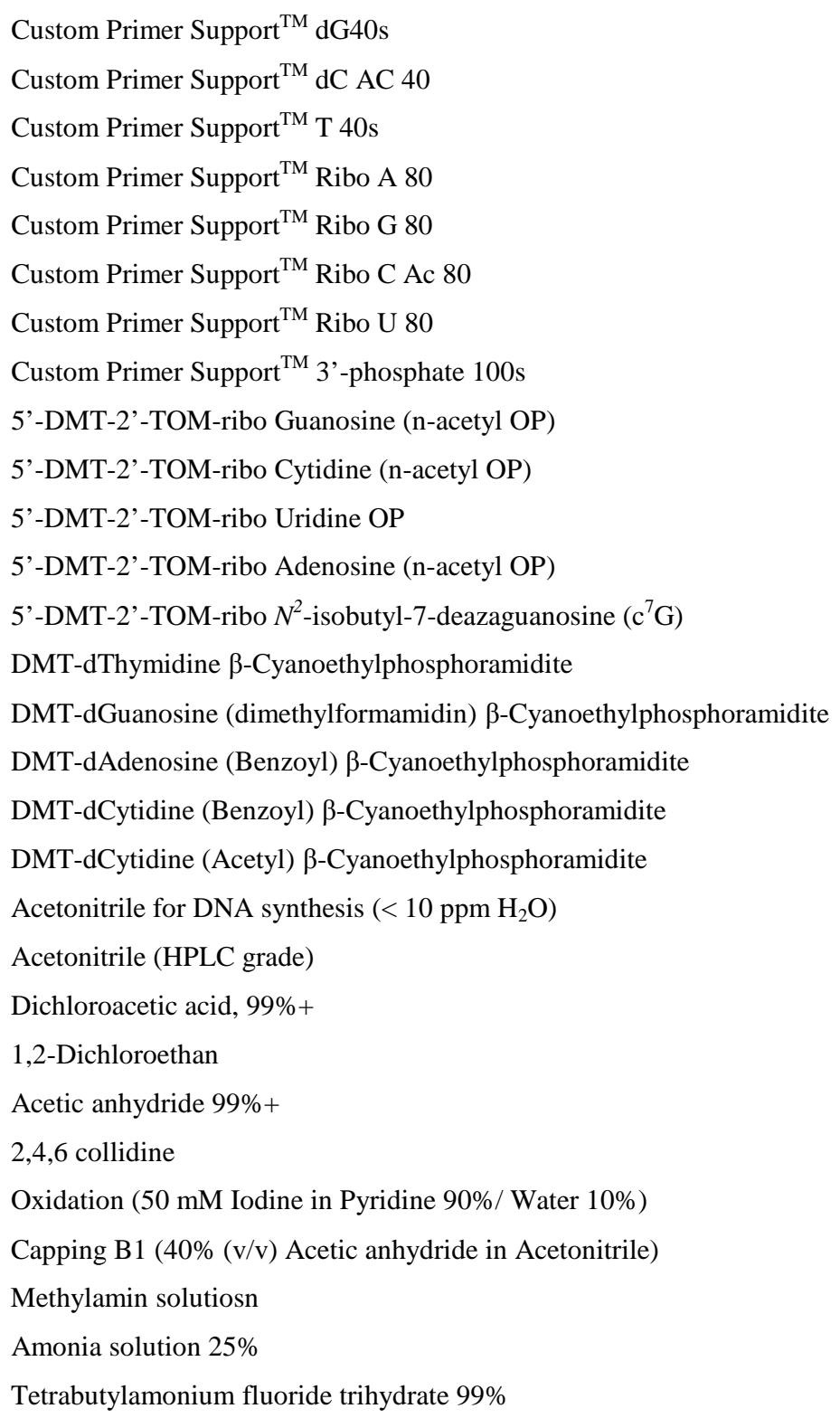

GE Healthcare
GE Healthcare
GE Healthcare
GE Healthcare
GE Healthcare
GE Healthcare
GE Healthcare
GE Healthcare
ChemGenes
ChemGenes
ChemGenes
ChemGenes
ChemGenes
SAFC
SAFC
SAFC
SAFC
SAFC
Sigma-Aldrich
Sigma-Aldrich
Acros organics
Roth
Acros organics
Acros organics
Amersham Bioscience
Amersham Bioscience
Aldrich
Merck
Acros organics


Tetrahydrofuran (water $<50 \mathrm{ppm}$ )

Iodine

5-Benzylthio-1H-tetrazole (BTT)

4-Dimethylamino-pyridin (DMAP)
Acros organics

Acros organics

Carbosynth

Fluka

\subsubsection{Instruments}

LKB. Gene Assembler Plus

Pharmacia

392 DNA/RNA synthesizer
Applied Biosystems

\subsubsection{Solutions for solid phase synthesis of oligonucleotides}

Oxidation, Cap A and Cap B solutions were purchased or alternatively prepared as is described here. Detritylation and activation solutions were only prepared manually.

$\begin{array}{ll}\text { Cap A } & 0.5 \mathrm{M} \text { 4-dimethylamino-pyridin (DMAP) in acetonitrile } \\ \text { Cap B } & \text { acetic anhydride/2,4,6-collidine/acetonitrile 20/30/50 } \\ \text { Oxidation } & 10 \mathrm{mM} \mathrm{I}_{2} \text { in acetonitrile/2,4,6-collidin/ } \mathrm{H}_{2} \mathrm{O} \text { 10/1/5 } \\ \text { Detritylation } & 3 \% \text { dichloroacetic acid (DCA) in 1,2-dichloroethane } \\ \text { Activation } & 0.25 \mathrm{M} \text { benzylthiotetrazole (BTT) in acetonitrile }\end{array}$

\subsubsection{Solid phase synthesis and deprotection}

Standard DNA and RNA 3'-ß-cyanoethyl diisopropylamino phosphoramidites were 5'-O-4,4'dimethoxytrityl (DMT) protected. Phosphoramidites have been purchased from ChemGenes, Wilmington, USA, or SAFC, Saint Louis, US, or have been synthesized by Dr. Falk Wachowius at Research Group Nucleic acid Chemistry, Max Planck Institute for Biophysical Chemistry, Göttingen. Phosphoramidite solutions (100 mM) were prepared by dissolving the phosphoramidites in absolute acetonitrile $\left(\leq 10 \mathrm{ppm} \mathrm{H}_{2} \mathrm{O}\right.$ ). Phosphoramidite solutions used for the CoMA and dNAIM were mixtures of parent $100 \mathrm{mM}$ dN phosphoramidite with $100 \mathrm{mM}$ (modified-) rN phosphoramidite with the ratio of 90:10. For each coupling step $75 \mu \mathrm{l}$ of the solution was used. Activator solution, BTT was prepared with low water content acetonitrile to the final concentration of $250 \mathrm{mM}$. Each coupling step required $250 \mu \mathrm{l}$ of the BTT solution. Both phosphoramidite and BTT solutions were dried overnight using activated molecular sieves. Molecular sieves ( $4 \AA$ ) were previously washed with water, dried at $110^{\circ} \mathrm{C}$ for at least $48 \mathrm{~h}$ followed by heating under high vacuum for at least $24 \mathrm{~h}$.

RNA or DNA oligonucleotides were synthesized on polystyrene primer supports on Pharmacia Gene Assembler Plus with $0.7 \mu \mathrm{mol}$ synthesis scales. Loading density for DNA and RNA solid supports were 40 and $80 \mu \mathrm{mol} / \mathrm{g}$ respectively. For $0.7 \mu \mathrm{mol}$ scale synthesis ca. 20 and ca. $10 \mathrm{mg}$ supports were used respectively for DNA and RNA synthesis. Most of the syntheses were in "trityl-off" mode, cleaving the terminal DMT group off at the final step, except otherwise was required.

Synthesis started with detrtiylation of the solid support using 3\% Dichloroacetic acid (DCA) in 1,2Dichloroethane for 2 min. After detritylation step dicloroethane wash and acetonitrile wash were 
performed generously. The coupling time for DNA and RNA phosphoramidites were 2 and 4 min respectively in presence of activation solution (0.25 M BTT in acetonitrile). Capping solutions A and B were mixed 1:1 and incubated for 1 min before and after oxidation step. Iodine mediated oxidation was accomplished in presence of oxidation solution for $1 \mathrm{~min}$. The solid phase was rinsed with low water content acetonitrile in between coupling, capping and oxidation steps.

DNA oligonucleotides were deprotected by $16 \mathrm{~h}$ incubation at $55^{\circ} \mathrm{C}$ with $1 \mathrm{ml}$ mixture of $\mathrm{NH}_{4} \mathrm{OH}$ : ethanol 3:1. RNA oligonucleotides were deprotected by 4-6 h incubation at room temperature or alternatively at $37^{\circ} \mathrm{C}$ with $1 \mathrm{ml}$ mixture of methylamine in $\mathrm{H}_{2} \mathrm{O}$ (41\%): methylamine in ethanol (33\%) 1:1. For DNA and RNA oligonucleotides with acetyl protection group on cytidines samples were incubated with AMA (1:1 mixture of $\mathrm{NH}_{4} \mathrm{OH}$ : methylamine in $\left.\mathrm{H}_{2} \mathrm{O}(41 \%)\right)$ for $2 \mathrm{~h}$ at $65^{\circ} \mathrm{C}$.

Samples were filtered and the dried under vacuum. DNA samples were dissolved in water and collected. RNA samples were further incubated with $600 \mu \mathrm{l}$ of $1 \mathrm{M} \mathrm{TBAF}$ in THF at $40^{\circ} \mathrm{C}$ for 5 hours for removal of the 2'-OH protection group (TOM). Deprotection was finished by addition of same volume of TrisHCl $1 \mathrm{M}$, pH 8.0. THF was evaporated and samples were desalted using size exclusion chromatography ( $\mathrm{H}_{2} \mathrm{O}$ as solvent, flow rate $\left.2 \mathrm{ml} / \mathrm{min}\right)$. Sample were collected, dried and dissolved in water.

\subsection{RNA in vitro transcription}

For in vitro transcription 100 pmol single stranded DNA template containing T7 RNA promoter complement, 100 pmol T7 RNA promoter were annealed in $50 \mu \mathrm{l}$ and in presence of transcription annealing buffer (15 mM NaCl, $4 \mathrm{mM}$ Tris- $\mathrm{HCl} \mathrm{pH}$ 8.0, $0.1 \mathrm{mM}$ EDTA) by 2 min heating at $95^{\circ} \mathrm{C}$ and at least $15 \mathrm{~min}$ incubation at room temperature. Buffer and NTPs were added to reach final concentration of $40 \mathrm{mM}$ Tris- $\mathrm{HCl} \mathrm{pH}$ 8.0, $30 \mathrm{mM} \mathrm{MgCl}_{2}, 10 \mathrm{mM}$ DTT, $2 \mathrm{mM}$ spermidine, $4 \mathrm{mM}$ of each NTP in the final volume of $100 \mu \mathrm{l} .2 \mu \mathrm{l}$ of non-commercial T7 RNA polymerase were added to start the transcription. Samples incubated at $37^{\circ} \mathrm{C}$ for $5 \mathrm{~h}$. EDTA $(100 \mathrm{mM})$ was added to the samples to stop the reaction. For experiments with phosphorothioate donor, $5^{\prime}-\gamma$ (or $\alpha$ )-thio-triphosphorylated acceptor RNA were transcribed in vitro using the same protocol and suing guanosine- 5'-( $\gamma$-thio)triphosphate or guanosine- 5'-( $\alpha$-thio)-triphosphate from Jena bioscience.

DNA templates for short RNA transcript (<60 nucleotides) were purchased from Sigma Aldrich. DNA templates for long RNA transcripts i.e. U6 snRNA (121 nucleotides long) were prepared by PCR amplification of a plasmid containing yeast U6 snRNA. Plasmid containing U6 RNA of Saccharomyces cerevisiae with a T7 promoter (pT7 U6 S.cer.) was provided by Olexandr Dybkov, department of Cellular Biochemistry, Max Planck Institute for Biophysical Chemistry. PCR reaction with primers D027 (T7 promoter) and D138 (reverse primer including BamHI restriction site) and the DNA template pT7 U6 yielded the transcription template for U6 snRNA. An extra G in the 5' end and 8 additional nucleotides on the 3' end were introduced to the sequence to improve transcription efficiency. 


\subsection{Gel purification and ethanol precipitation}

Denaturing $10-20 \%$ polyacrylamide gel containing $7 \mathrm{M}$ urea were prepared by standard protocol ${ }^{212}$ using urea (from Merck), Tris, Boric acid and Rotiphorese gel 40 (from Carl Roth). Crude synthesized oligonucleotides and in vitro transcribed RNAs were purified by denaturing polyacrylamide gel electrophoresis containing $7 \mathrm{M}$ urea and 1x TBE (89 mM Tris-HCl pH 8.0, $89 \mathrm{mM}$ Boricacid, $2 \mathrm{mM}$ EDTA pH 8.0). The desired bands were cut and extracted. Sliced pieces of the gel was incubated with TEN buffer (10 mM Tris-HCl pH 8.0, 1 mM EDTA pH 8.0 and $300 \mathrm{mM} \mathrm{NaCl}$ ) for 1.5 hour at $37^{\circ} \mathrm{C}$ or overnight at room temperature. The extraction was performed two times for higher efficiency. The oligonucleotides in the supernatant were precipitated with ethanol. Three volumes of ethanol was added to the samples, samples incubated at $-20^{\circ} \mathrm{C}$ for $2 \mathrm{~h}$ and centrifuged at $13200 \mathrm{rpm}$ for $30 \mathrm{~min}$ at $4^{\circ} \mathrm{C}$. Supernatants of the samples were removed and the pellets were washed once or twice with $70 \%$ ethanol, continued by at least $10 \mathrm{~min}$ further centrifugation at $4^{\circ} \mathrm{C}$ and removal of the supernatant. The concentration of the samples were measured using Nanophotometer from IMPLEN. Pellets were dried and dissolved in 40-100 $\mu \mathrm{l}$ water. The purity of the samples was controlled by HPLC.

\subsection{PCI extraction}

To $100 \mu \mathrm{l}$ of aqueous solution containing mixture of oligonucleotides and proteins, $100 \mu \mathrm{l}$ of phenolchloroform-isoamyl alcohol 25:24:1 (Roth) was added. Sample was mixed thoroughly for 1 min and centrifuged for $1 \mathrm{~min}$ to separate the two phases. Aqueous phase (top) was removed to a new tube. Phenol phase was washed with $50 \mu \mathrm{H}_{2} \mathrm{O}$. To the $150 \mu \mathrm{l}$ of the aqueous phase $100 \mu \mathrm{l}$ chloroformisoamyl alcohol (Roth) was added. Samples were mixed thoroughly and centrifuged to separate the two phases. The aqueous phase was removed to a new tube. The chloroform-isoamyl alcohol phase was washed with additional $50 \mu \mathrm{l}$ of $\mathrm{H}_{2} \mathrm{O}$. Extracted oligonucleotides in aqueous phase (200 $\left.\mu \mathrm{l}\right)$ were precipitated by addition of $20 \mu \mathrm{l} 3 \mathrm{M} \mathrm{NaCl}$ and $600 \mu \mathrm{l}$ of absolute cold ethanol. Ethanol precipitation was perused as described above.

\subsection{HPLC analysis}

Äkta purifier (GE healthcare) was used for HPLC analyses of this report. Anion-exchange chromatography on a Dionex DNAPac PA200 Analytical column was performed by increasing gradient of eluent B (25 mM Tris- $\mathrm{HCl} \mathrm{pH}$ 8, $0.5 \mathrm{M} \mathrm{NaClO}_{4}, 6 \mathrm{M}$ urea,) in eluent A (25 mM Tris-HCl pH 8.0, 6 $\mathrm{M}$ urea). All the HPLC analyses have been performed at $80^{\circ} \mathrm{C}$. For analysis of the quality of the synthesized and purified RNA and DNA oligonucleotides $25 \mu \mathrm{l}$ sample (containing ca 100 pmol nucleic acid) were injected by auto-sampler and eluted with a gradient of $0-60 \%$ eluent B in eluent A in 45 min at $80^{\circ} \mathrm{C}$. HPLC analysis of the fate of triphosphate donor RNA in presence of $\mathrm{Tb}^{3+}$ started with $25 \%$ eluent B in A for 1 column volume (CV), percentage of B increased to 50\% in $8 \mathrm{CV}$. Column was washed for 1 CV with $100 \%$ eluent B and additional 2 CV with $100 \%$ eluent A. For HPLC analysis of 
preparative ligation reactions, HPLC started with 35\% eluent B which increased to the target percentage of 100 in 12 CV. Additional wash with 2 CV of $100 \%$ eluent A was the last step.

\subsection{Enzymatic phosphorylation of 5'ends}

T4 polynucleotide kinase (PNK) enzyme was used to phosphorylate 5' ends with free hydroxyl groups. 5'-OH oligonucleotides (0.5-2 nmol) were incubated in presence of 1x PNK buffer (50 mM Tris-HCl, pH 7.6, $10 \mathrm{mM} \mathrm{MgCl}$, 5 mM DTT, 0.1 mM spermidine, 0.1 mM EDTA) and $1 \mathrm{mM}$ ATP in 30-40 $\mu \mathrm{l}$ at $37^{\circ} \mathrm{C}$ for 3 h. Samples were ethanol precipitated afterwards to remove non-incorporated ATPs. For radiolabeling reactions, $50 \mathrm{pmol} 5$ '-OH oligonucleotides were incubated in presence of $1 \mathrm{x}$ PNK buffer and $\gamma$-32P-ATP (5 $\mu \mathrm{Ci}$ ) in $10 \mu \mathrm{l}$ at $37^{\circ} \mathrm{C}$ for $1 \mathrm{~h}$. Radiolabelled samples were loaded on a $0.4 \mathrm{~mm}$ thick 20\% denaturing PAGE for $45 \mathrm{~min}$ and extracted as described above. Alternatively, the reaction mixtures were passed through a MicroSpin G-25 column to remove the unincorporated $\gamma-{ }^{32} \mathrm{P}$-ATP following the company’s suggested protocol. The procedure continued by phenol-chloroform-isoamyl alcohol (PCI) extraction and completed by ethanol precipitation.

For 5'-radio labeling of RNA transcript, first a dephosphorylation step was performed using calf intestinal alkaline phosphatase (CIAP) or antarctic alkaline phosphatase. The free 5'-OH then was subjected for phosphorylation reaction by PNK. Dephosphorylation by CIAP was performed for 0.2-1 nmol of transcript in presence of 1x CIAP buffer (50 mM Tris-HCl pH 8.5, $0.1 \mathrm{mM}$ EDTA) and 5 units of CIAP enzyme from Fermentas in $30 \mu \mathrm{l}$ at $37^{\circ} \mathrm{C}$ for $3 \mathrm{~h}$. Dephosphorylation by antarctic alkaline phosphatase was performed for 50-200 pmol of transcript in presence of $1 \mathrm{x}$ antarctic buffer (50 mM Bis-Tris-HCl-Propane- $\mathrm{HCl}, 1 \mathrm{mM} \mathrm{MgCl}, 0.1 \mathrm{mM} \mathrm{ZnCl}_{2}, \mathrm{pH}$ 6) using 5 units of antarctic phosphatase enzyme from New England Biolabs. (NEB) in $30 \mu \mathrm{l}$ at $37^{\circ} \mathrm{C}$ for 30 min. For both CIAP and antarctic alkaline phosphatase, the transcript was extracted by PCI and ethanol precipitation prior to PNK reaction.

\subsection{Enzymatic radiolabeling of 3' ends}

Labeling of the 3' ends of DNAs have been performed using Klenow 3' $\rightarrow$ 5' exo' from Jena Bioscience. A short DNA strand (splint, ca 20 nucleotide long) was hybridized to the 3' end of the DNA and served as a template for Klenow dependent strand extension. DNA (50 pmol) and splint (300-500 pmol) were annealed in $12 \mu \mathrm{l}$ by heating to $95^{\circ} \mathrm{C}$ for $2 \mathrm{~min}$ and further incubation at room temperature for 15 min. Klenow buffer (final 1x concentration: $10 \mathrm{mM}$ Tris-HCl pH 7.5, $5 \mathrm{mM} \mathrm{MgCl}$, $7.5 \mathrm{mM}$ DTT), $\alpha-{ }^{32} \mathrm{P}$ dATP $\left(5 \mu \mathrm{Ci}\right.$ ) and 1 unit Klenow fragment were added. Samples were incubated at $37^{\circ} \mathrm{C}$ for 3 hours. After formation of a phosphodiester bond between 3' end of DNA and the $\alpha$ - phosphate of the dATP by Klenow, the 3' $\alpha-{ }^{32} \mathrm{P}$-labeled reaction products were isolated by purification on a $12 \%$ denaturing PAGE to separate splint and non-incorporated $\alpha-{ }^{32} \mathrm{P}$ dATP from radiolabeled oligonucleotides. 


\subsection{Standard annealing procedure}

Annealing of oligonucleotides was performed before catalytic assays, spectroscopic investigations and DNase or DMS probing assays. Nucleic acid components were incubated for $2 \mathrm{~min}$ at $95^{\circ} \mathrm{C}$ in presence of annealing buffer (5 mM HEPES pH 7.5, $15 \mathrm{mM} \mathrm{NaCl}, 0.1 \mathrm{mM}$ EDTA). Samples were annealed at room temperature for at least $15 \mathrm{~min}$.

\subsection{T4 RNA ligation}

To ligate acceptor RNAs to CoMA, dNAIM and DMSi libraries, 5' phosphorylated DNA molecules (1 nmol) were annealed with acceptor RNA (2 nmol). T4 RNA ligation buffer (50 mM Tris pH 7.5, 10 mM DTT, $5 \mathrm{mM} \mathrm{MgCl}_{2}$ ), ATP (0.5 mM) and T4 RNA ligase from Fermentas (10 units) were added and volume was adjusted to $30 \mu \mathrm{l}$. Samples incubated at room temperature for overnight. The ligation products were purified after 10\% denaturing PAGE by extraction and ethanol precipitation.

\subsection{Combinatorial mutation interference analysis (CoMA)}

CoMA consists of 4 steps: 1) solid phase synthesis of the mutant libraries, 2) separation of the active mutants, 3) alkaline hydrolysis and 4) PAGE analysis ${ }^{139}$. Experimental details of CoMA analysis of the deoxyribozymes used in this report were as follows.

Step1: Four mutant libraries (rA, rC, rG, rU) were synthesized on solid supports using the standard solid phase synthesis protocol with slight modifications. The deoxyribozymes' binding arms were synthesized with solutions of $\mathrm{dN}$ phosphoramidites. The 3' binding arms were synthesized in "trityl-on" mode. Syntheses were continued using dN:rN phosphoramidite mixtures 90:10 for the catalytic core of the deoxyribozymes. The syntheses of the catalytic cores were in "trityl-on" mode as well. The final steps were syntheses of the 5' binding arms with standard dN phosphoramidites in "trityl-off" mode.

Step2: DNA libraries were phosphorylated at 5' end. The 5' phosphorylated libraries have been ligated to the acceptor RNA using T4 RNA ligase. The conjugated donor RNA and DNA libraries were radiolabeled at 3' end using Klenow fragment and 6-10 times excess of DNA splint. Ideally the splint DNA should not be extended and therefore radiolabeled i.e. the DNA library binding arm should not serve as a template for $\alpha-{ }^{32} \mathrm{P}$-dATP attachment to the splint. To avoid any radiolabeling of the splint a 5'-phosphorylated splint was used instead of 5'-OH containing splint. 5'-phosphorylated splint was synthesized on solid phase having a DMT-protected phosphate group as initiator building block for DNA synthesis.

The time and conditions are crucial for the ligation reaction of CoMA separation step. Deoxyribozyme ligation for CoMA of 6BX22, 9F13 and 9F7 were in presence of $50 \mathrm{mM}$ HEPES pH 7.5, $150 \mathrm{mM} \mathrm{NaCl}$, $2 \mathrm{mM} \mathrm{KCl}$ and millimolar concentrations of a bivalent metal ion as active metal ion and in cases 
micromolar concentrations of $\mathrm{TbCl}_{3}$. The catalytic step of the enzyme was performed with $50 \mathrm{pmol}$ of donor RNA-DNA library and equimolar amount of the acceptor RNA (in the case of 9F7) or excess of the acceptor RNA (60 pmol in the case of 6BX22 and 9F13). The reaction volumes were 50-70 $\mu$ l. The reaction times were between $20 \mathrm{~min}$ and $10 \mathrm{~h}$. The reaction times were selected based on the kinetics of the enzyme at different condition (table 5.11-1).

Table 5.11-1 Reaction time and conditions for separation step of CoMA

\begin{tabular}{llll} 
deoxyribozyme & Condition & volume $(\mu \mathrm{l})$ & time \\
\hline 9F7 & $20 \mathrm{mM} \mathrm{Mn}^{2+}$ & 60 & $20 \mathrm{~min}$ \\
9F7 & $2 \mathrm{mM} \mathrm{Mn}^{2+}$ & 70 & $10 \mathrm{~h}$ \\
9F7 & $2 \mathrm{mM} \mathrm{Mn}^{2+}+10 \mu \mathrm{M} \mathrm{Tb}^{3+}$ & 70 & $1 \mathrm{~h}$ \\
9F7 & $60 \mathrm{mM} \mathrm{Mg}^{2+}$ & 60 & $8 \mathrm{~h}$ \\
9F7 & $80 \mathrm{mM} \mathrm{Mg}^{2+}+100 \mu \mathrm{M} \mathrm{Tb}^{2+}$ & 60 & $2 \mathrm{~h}$ \\
6BX22 & $20 \mathrm{mM} \mathrm{Mn}^{2+}$ & 60 & $0.5-5 \mathrm{~h}$ \\
9F13 & $20 \mathrm{mM} \mathrm{Mn}^{2+}$ & 60 & $3 \mathrm{~h}$
\end{tabular}

Step3: The active and inactive fractions were separately subjected to alkaline hydrolysis. $2 \mu \mathrm{l}$ sample was incubated with $2 \mu \mathrm{l} 20 \mathrm{mM} \mathrm{NaOH}$ and incubated at $95^{\circ} \mathrm{C}$ for $10 \mathrm{~min}$. Heating was performed using an Eppendorf Mastercycler. The temperature for lid and samples were set at $95^{\circ} \mathrm{C}$. The heating from the lid avoids evaporation and increasing $\mathrm{NaOH}$ concentration.

Step4: The hydrolyzed samples were loaded on 20\% denaturing PAGE for ca. 4h at 35W. After electrophoresis the gel was dried on a 3MM chromatography paper (Whatman) in gel dryer (Bio-RAD) at $80^{\circ} \mathrm{C}$ for $30 \mathrm{~min}$ and exposed to a storage phosphor screen (GE healthcare) overnight. Scanner STORM 820 GE Healthcare was used to scan the phosphorimager screens. Storage phosphor screen were erased afterwards using Image eraser (Amersham Bioscience). ImageQuant 5.2 was used to quantify the band intensities. The borders of each band were defined manually by drawing a box around each band. For each band, background was deducted by the intensity of another box with the same size. Band intensities were normalized to eliminate loading differences. One of the positions which accepted all mutations under the tested conditions was selected for normalization (Pos. ${ }^{\text {norm }}$ ). Normalization factor was defined as a factor which makes the intensity of Pos. ${ }^{\text {norm }}$ of all lanes equal to each other. Normalization factor for each lane was calculated as the ratio of the Pos. ${ }^{\text {norm }}$ intensity of that lane to the pos. $^{\text {norm }}$ of a reference lane. Usually active rG library was selected as the reference lane. All the bands of each lane were divided by the normalization factors of the respective lane. After normalization, interference values were calculated as the ratio of the unselected library over active library at the same band position. The interference values below 2 were considered as non-significant. 


\subsection{2 dNAIM}

$\mathrm{dNAIM}^{140}$ and DMSi ${ }^{39}$ have been performed on trimmed 6BX22. For dNAIM four DNA libraries of (modified) guanosines have been synthesized. The libraries were as follows: $d G / r G, d G / r-c^{7} G, d G / r-2$ aminopurine and $\mathrm{dG} / \mathrm{r}-\mathrm{m}^{1} \mathrm{G}$. For each library a mixture of $90: 10 \mathrm{dG} / \mathrm{rG}^{\mathrm{mod}}$ phosphoramidite has been made and used wherever $\mathrm{G}$ is present in the sequence. With each $\mathrm{dG} / \mathrm{rG}^{\text {mod }}$ mixture a pentamer has been synthesized to control $\mathrm{rG}^{\text {mod }}$ incorporation. To have statistically one $\mathrm{rG}^{\text {mod }}$ distributed in the catalytic core of the trimmed 6BX22 5\% incorporation of $\mathrm{rG}^{\mathrm{mod}}$ was desired.

The phosphorylated DNA libraries were ligated the donor RNA (Tr03) as explained in CoMA. Donor RNA- DNA libraries (60 pmol) were radio-labeled with $\alpha-{ }^{32} \mathrm{P}$ dATP at their 3' end. To perform the 2',5' branch formation reaction and separation of active fractions, the labeled donor RNA-DNA libraries incubated with 120 pmol acceptor RNA (R01) in presence of 6BX22 buffer i.e. 50 mM HEPES pH 7.5, $50 \mathrm{mM} \mathrm{NaCl}, 2 \mathrm{mM} \mathrm{KCl}$ and $20 \mathrm{mM} \mathrm{MnCl}_{2}$ at $37^{\circ} \mathrm{C}$ for 3 hours. Active fractions, inactive fractions and unseparated libraries were hydrolyzed and analyzed as in CoMA.

To prepare the $\mathrm{m}^{7} \mathrm{G}$ library for DMSi, 2 nmol trimmed 6BX22 was annealed with $2.5 \mathrm{nmol}$ of inactivated donor RNA (R005) and inactivated acceptor RNA (R02) in $50 \mu$ l. To the annealed sample, 1 $\mu \mathrm{l}$ of 5\% DMS was added. Samples were mixed and spin down and incubated for $1 \mathrm{~h}$ at room temperature. Methylation reaction was stopped by addition of $40 \mu \mathrm{l}$ of DMS stop solution. DNA was precipitated, phosphorylated at 5' end and further ligated to the acceptor RNA (Tr03) using T4 RNA ligase. Separation of active fraction was performed like other dNAIM libraries. Modification sites were visualized by piperidine cleavage and further PAGE analysis as it was done for other dNAIM libraries. Piperidine cleavage was performed by addition of $50 \mu \mathrm{l} 10 \%$ Piperidine and further incubation at $90^{\circ} \mathrm{C}$ for 30 min continued by evaporation of Piperidine in speedvac.

\subsection{Kinetic and preparative reactions of deoxyribozymes}

The suppliers of buffers and metal ions used for kinetic experiments were as follows.

$\begin{array}{ll}\text { 4-(2-Hydroxyethyl)piperazine-1-ethanesulfonic acid (HEPES) } & \text { Roth } \\ \text { 2-(Cyclohexylamino)-ethansulfonic acid (CHES) } & \text { Roth } \\ \text { Sodium chloride } & \text { Merck } \\ \text { Potassium chloride } & \text { Merck } \\ \text { Magnesium chloride hexahydrate } & \text { Merck } \\ \text { Manganese (II) chloride 99+\% } & \text { Aldrich } \\ \text { Lanthanum (III) chloride heptahydrate 99.999\% } & \text { Aldrich } \\ \text { Cerium (III) chloride heptahydrate 99.9\% } & \text { Aldrich } \\ \text { Europium (III) chloride hexahydrate 99.99\% } & \text { Acros organics } \\ \text { Gadolinium (III) chloride hexahydrate 99.999\% } & \text { Aldrich } \\ \text { Terbium (III) chloride hexahydrate 99.9\% } & \text { Acros organics } \\ \text { Ytterbium (III) chloride hexahydrate 99.9\% } & \text { Aldrich }\end{array}$


6BX22 standard kinetics experiments have been performed in three-molecular format using 5 pmol acceptor (R001, 1 pmol 5, ${ }_{-32}$ P-radiolabelled RNA and 4 pmol non-radiolabeled RNA), 15 pmol trimmed 6BX22 (D010) and 30 pmol donor RNA (Tr03). Nucleic acid components of the reactions were annealed with standard procedure. 5x HEPES mix buffer (250 mM HEPES pH 7.5, $750 \mathrm{mM} \mathrm{NaCl,} 10$ $\mathrm{mM} \mathrm{KCl}$ ) added to the samples to reach final concentration of $50 \mathrm{mM}$ HEPES pH 7.5, $150 \mathrm{mM} \mathrm{NaCl}$ and $2 \mathrm{mM} \mathrm{KCl}$. The reaction started by addition of $1 \mu \mathrm{l}$ of $10 \mathrm{x} \mathrm{MnCl}_{2}$ or the mixture of indicated metal ions. Standard reaction volume for kinetic assays was $10 \mu \mathrm{l}$. aliquots $(0.7 \mu \mathrm{l})$ of reactions were quenched by addition of $2 \mu \mathrm{l}$ of stop solution (80\% formamide, 1x TBE, 50 mM EDTA, $0.0025 \%$ each of bromophenol blue and xylene cyanol). Samples were kept on dry ice before loading on $15 \%$ denaturing PAGE for $45 \mathrm{~min}$ at $35 \mathrm{~W}$. The gel dried and exposed to Phophorimager screen. The band intensities were quantified by ImageQuant. After background correction the ratio of the product band to the substrate RNA was calculated and plotted against time. The kinetic curves were fit to the exponential fit formula $Y=Y_{\max }\left(1-e^{-k_{o b s} \cdot t}\right)$ or to the linear fit whenever suitable. $Y=k_{o b s} \cdot t$

For 6BX22 chase experiment, the reaction started with standard concentrations of substrates and metal ion in $30 \mu$ l. single and double chase experiments started with $10 \mathrm{mM} \mathrm{Mn}^{2+}$. $\mathrm{Tb}^{3+}$ (up to $100 \mu \mathrm{M}$ ) and additional $\mathrm{Mn}^{2+}$ (up to $20 \mathrm{mM}$ ) were added after $20 \mathrm{~min}$ and $60 \mathrm{~min}$ of start of the reaction. For control

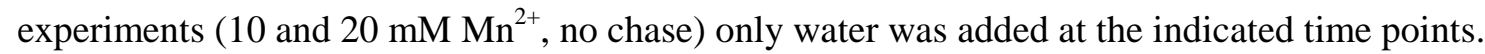

Standard kinetic experiments for 9F7 or 9F13 were carried out in three-molecular format and in $10 \mu \mathrm{l}$ volume. 1 pmol 5'-radiolabeled acceptor RNA mixed with 4 pmol non-radiolabeled acceptor RNA, 15 pmol 9F7 DNA and 30 pmol donor RNA and annealed in $6 \mu$ l. standard acceptor and donor RNAs for 9F7 and 9F13 were R012 and Tr12 respectively. Standard original length 9F7 was D046. Reactions were performed in presence of $50 \mathrm{mM}$ HEPES pH 7.5, $150 \mathrm{mM} \mathrm{NaCl}, 2 \mathrm{mM} \mathrm{KCl}$ and $20 \mathrm{mM} \mathrm{MnCl}_{2}$ or indicated concentration of other metal ions at $37^{\circ} \mathrm{C}$. In cases that $\mathrm{Tb}^{3+}$ is present in the reaction, $10 \mathrm{x}$ $\mathrm{Tb}^{3+}$ and $10 \mathrm{x} \mathrm{M}^{2+}$ solutions were premixed prior to addition to the reaction. The reaction times varied between 20 min to 24 hours. Samples were separated and analyzed as explained for 6BX22 kinetics.

Kinetic investigations with 10DM24 were performed by annealing of 2 pmol acceptor RNA (1 pmol 5'radiolabeled and 1 pmol non-radiolabeled RNA), 10 pmol 10DM24 deoxyribozyme and 20 pmol of either donor RNA or helper RNA (in the case of GTP attachment) in 4-6 $\mu$ l volume. In cases of GTP attachment, GTP served as the donor substrates. GTP (2 nmol) was added after the annealing step and before addition of reaction buffer and metal ions. Then $5 x$ HEPES mix buffer was added to the sample to reach final concentrations of $50 \mathrm{mM}$ HEPES, $150 \mathrm{mM} \mathrm{NaCl}$ and $2 \mathrm{mM} \mathrm{KCl}$ respectively. Reactions were started by addition of $80 \mathrm{mM} \mathrm{MgCl}_{2}$, except otherwise is mentioned. For experiments with $\mathrm{Tb}^{3+}$ mediated acceleration $10 \mathrm{x} \mathrm{MgCl}_{2}$ and $10 \mathrm{x} \mathrm{TbCl}_{3}$ were premixed prior to addition to the reaction. Standard condition for acceleration effect was in presence of $80 \mathrm{mM} \mathrm{MgCl}_{2}$ and $100 \mu \mathrm{M} \mathrm{Tb}^{3+}$. 
For preparative (modified-)GTP attachment to SAM III 3' fragment, substrate (Tr18), GTP attaching 10DM24 (D121) and helper RNA (R14) were annealed in $50 \mu$ l. (modified)-GTP, buffer and metal ions were added sequentially. The reaction volume was adjusted to $100 \mu \mathrm{l}$ to reach the final concentrations of 5:6:8 $\mu \mathrm{M}$ of substrate: DNA: helper RNA, $20 \mu \mathrm{M}$ GTP, $50 \mathrm{mM}$ HEPES pH 7.5, $150 \mathrm{mM} \mathrm{NaCl}, 2 \mathrm{mM}$ $\mathrm{KCl}, 80 \mathrm{mM} \mathrm{MgCl}$ and $100 \mu \mathrm{M} \mathrm{TbCl}_{3}$. The $10 \mathrm{x}$ concentrated $\mathrm{MgCl}_{2}$ and $\mathrm{TbCl}_{3}$ were pre-mixed prior to addition to the reaction. The samples were incubated $20-30 \mathrm{~min}$ at $37^{\circ} \mathrm{C}$. The higher amount of oligonucleotide loaded on a gel volume leads to the higher extraction efficiency. Therefore, after the reaction two samples (each containing 500 pmol RNA) were mixed, ethanol precipitated and loaded on one pocket of 0.8 well $0.4 \mathrm{~mm}$ thick gel. The bands containing helper RNA, 10DM24 DNA and labeled RNA were cut and the oligonucleotides were extracted. 10DM24 enzymes and helper RNAs could be used for a new labeling reaction.

To produce full length SAM III, SAM III 5' fragment (R35) and 3' fragment (Tr18) were ligated by minimized version of 9DB1 deoxyribozyme. 9DB1 catalyzes the nucleophilic attack of the 3'-OH of the acceptor RNA to the $\alpha$ phosphate of the donor RNA strand to form a natural linear 3'-5' phosphodiester bond. Here the acceptor RNA strand was synthesized by solid phase synthesis. Triphosphorylated donor was prepared by in vitro transcription. Equimolar ratios of minimized 9DB1 deoxyribozyme ${ }^{139}$, donor and acceptor RNA (2 nmol of each) were incubated in presence of $150 \mathrm{mM} \mathrm{NaCl}, 2 \mathrm{mM} \mathrm{KCl}, 40 \mathrm{mM}$ $\mathrm{MgCl}_{2}, 50 \mathrm{mM}$ CHES buffer at $\mathrm{pH} 9.0$ in $150 \mu \mathrm{l}$ at $37^{\circ} \mathrm{C}$ for $5 \mathrm{~h}$. The reaction mixtures were loaded on 15\% denaturing PAGE, $0.4 \mathrm{~mm}$ spacer, $1 \mathrm{nmol}$ per lane. Electrophoresis was performed for $1 \mathrm{~h} 30$ at $35 \mathrm{~W}$.

For preparative scale formation of branched products by 6BX22, reactions were started with 1 nmol of donor RNA and equimolar or slight excess of DNA and acceptor RNA, incubated in the presence of 20 $\mathrm{mM} \mathrm{Mn}{ }^{2+}$ for $3 \mathrm{~h}$ at $37^{\circ} \mathrm{C}$ and resulted in $15 \%-60 \%$ recovery yield of the branched product.

\subsection{HPLC analysis of 9F7 kinetics}

For kinetic experiments of $1 \mu \mathrm{M}$ oligonucleotide complexes, acceptor RNA, 9F7 original DNA and donor RNA were mixed and annealed in $60 \mu$ l. Buffer and metal ions were added to reach the indicated metal ion concentrations in $100 \mu$ l. The samples incubated at $37^{\circ} \mathrm{C}$ for the stated time. Reactions were stopped by addition of $100 \mathrm{mM}$ EDTA. Ethanol precipitation was the next step. The pellets were washed one or two times by $70 \%$ ethanol to remove excess of metal ions. The precipitated pellet dissolved in $25 \mu \mathrm{l}$ of water prior to injection. For kinetic experiments of $10 \mu \mathrm{M}$ oligonucleotide complexes, the annealing step was done in $6 \mu \mathrm{l}$ and the final reaction volume was $10 \mu \mathrm{l}$ and no ethanol precipitation was required. Samples were injected into analytical HPLC (gradient: $1 \mathrm{CV}$ : 25\% eluent B, target concentration: $50 \%$ eluent $\mathrm{B}$ during $8 \mathrm{CV}$ ) and the area under $\mathrm{UV}^{260 \mathrm{~nm}}$ trace was integrated to calculated the ligation yield. 


\subsection{Circular dichroism (CD) measurements}

CD measurements have been performed with ChiraScan Applied Photophysics CD spectrometer. The spectra were measured in the range of 200-320 nm with acquisition time of 2.0 seconds. Scans were repeated at least 3 times. Sample volumes were $400 \mu$ l. Nucleic acid component concentration was 1.5 $\mu \mathrm{M}$. The measurements were all buffered with $50 \mathrm{mM}$ HEPES, $\mathrm{pH} 7.5$ and were done in presence of $150 \mathrm{mM} \mathrm{NaCl}, 2 \mathrm{mM} \mathrm{KCl}$ and $20 \mathrm{mM} \mathrm{MnCl}_{2}$. In case of $\mathrm{Tb}^{3+}$ titration, the raw data were normalized by correction for volume changes.

\subsection{Fluorescence spectroscopy of TMPyP4}

Meso-5,10,15,20-Tetrakis-(N-methyl-4-pyridyl)porphine, Tetratosylate (TMPyP4) was purchased from Calbiochem. TMPyP4 was dissolved in water and $500 \mu \mathrm{M}$ aliquots were stored in $-20^{\circ} \mathrm{C}$. Fluorescence spectra of the TMPyP4 were measured in presence of 6BX22 DNAzyme, the 6BX22 catalytic core only and unrelated DNA under standard reaction conditions in a volume of $120 \mu$ l. DNA titration (0-20 $\mu \mathrm{M}$ in 5 steps in presence of $2 \mu \mathrm{M}$ TMPYP4) has been done in excitation wavelength of $433 \mathrm{~nm}$ with the slit width of $5 \mathrm{~nm}$. Emission spectra were collected between 600 and $800 \mathrm{~nm}$, the emission slit width was set to $5 \mathrm{~nm}^{160}$. The measurements were done with Varian Cary Eclipse Fluorescence Spectrophotometer. PMT detector was set at $700 \mathrm{~V}$. TMPyP4 titration was performed at the same excitation and emission wavelengths in presence of $20 \mu \mathrm{M}$ DNAs.

\section{$5.17 \mathrm{~Tb}^{3+}$ luminescence spectroscopy}

Luminescence studies of 9F7 were performed using $1.2 \mathrm{nmol}$ trimolecular format oligonucleotide complexes. Samples were annealed in 50-60 $\mu$ l. Buffer and metal ions were added to reach final concentration of $50 \mathrm{mM}$ HEPES pH 7.5, $150 \mathrm{mM} \mathrm{NaCl}, 2 \mathrm{mM} \mathrm{KCl}$ and indicated divalent metal ion to the final volume of $600 \mu \mathrm{l}$. The final oligonucleotide complex concentrations were $2 \mu \mathrm{M}$. The complexes were incubated at room temperature for $1 \mathrm{~h}$ to equilibrate with the bivalent metal ion and fold properly prior to $\mathrm{Tb}^{3+}$ titration.

$\mathrm{Tb}^{3+}$ titration started by stepwise addition of $1 \mu$ laliquots of concentrated $\mathrm{Tb}^{3+}$ solutions according to the table 5.16-1. $\mathrm{Tb}^{3+}$ titration were performed with CARY Eclipse Fluorescence Spectrophotometer using Kinetics software. The excitation wavelength was at $284 \mathrm{~nm}$ (slit $10 \mathrm{~nm}$ ) and the emission was set on $545 \mathrm{~nm}$ (slit $10 \mathrm{~nm}$ ). The settings for the data collection were as follows: total decay time: $0.02 \mathrm{~s}$, number of flashes: 1 , delay time: $0.2 \mathrm{~ms}$, gate time: $5.0 \mathrm{~ms}$. The detector voltage was $600-800 \mathrm{~V}$ and was adjusted to signal strength. Data acquisition started 10-20 seconds after addition of each $\mathrm{Tb}^{3+}$ aliquots. For $9 \mathrm{~F} 7 \mathrm{~Tb}^{3+}$ titrations the luminescence intensities after 10 min equilibration time are plotted. The titration curves were fitted to the equation: $I=I_{\max } *\left[\mathrm{~Tb}^{3+}\right]^{\mathrm{n}} /\left(\left[\mathrm{Tb}^{3+}\right]_{1 / 2}{ }^{\mathrm{n}}+\left[\mathrm{Tb}^{3+}\right]^{\mathrm{n}}\right)$. 
For $\mathrm{Tb}^{3+}$ luminescence studies of 6BX22, final oligonucleotide complex concentrations were $2 \mu \mathrm{M}$. measurements were performed in presence of $50 \mathrm{mM}$ HEPES pH 7.5, $150 \mathrm{mM} \mathrm{NaCl}, 2 \mathrm{mM} \mathrm{KCl}$ and in presence or absence of $20 \mathrm{mM} \mathrm{MnCl}_{2}$ in $120 \mu \mathrm{l}$ at $25^{\circ} \mathrm{C} \mathrm{Tb}^{3+}$ titrations started by addition of $1 \mu \mathrm{l}$ of appropriate concentrated $\mathrm{Tb}^{3+}$ solutions. $\mathrm{Tb}^{3+}$ solution stocks were kept in $-20^{\circ} \mathrm{C}$.

Table 5.17-1 Preparation of stock solutions for $\mathrm{Tb}^{3+}$ titration. Concentrated stock solutions for each step were prepared by dilution of two parent stock solutions of 10 and $100 \mathrm{mM} \mathrm{TbCl}_{3}$.

\begin{tabular}{llllll}
\multirow{2}{*}{$\begin{array}{l}\text { Titration } \\
\text { step }\end{array}$} & desired $\mathrm{Tb}^{3+}$ & concentrated stock & \multicolumn{4}{l}{ To make the concentrated stock } \\
\cline { 5 - 6 } & concentration $(\mu \mathrm{M})$ & for each step $(\mu \mathrm{M})$ & Parent stock $(\mu \mathrm{M})$ & how much $(\mu \mathrm{l})$ & $\mathrm{H}_{2} \mathrm{O}(\mu \mathrm{l})$ \\
\hline 1 & 1.00 & 601 & 10000 & 30.05 & 469.95 \\
2 & 1.50 & 302 & 10000 & 15.1 & 484.9 \\
3 & 2.00 & 303 & 10000 & 15.15 & 484.85 \\
4 & 3.00 & 606 & 10000 & 30.3 & 469.7 \\
5 & 4.00 & 608 & 10000 & 30.4 & 469.6 \\
6 & 5.00 & 610 & 10000 & 30.5 & 469.5 \\
7 & 7.00 & 1219 & 10000 & 60.95 & 439.05 \\
8 & 10.00 & 1831 & 10000 & 91.55 & 408.45 \\
9 & 12.00 & 1228 & 10000 & 61.4 & 438.6 \\
10 & 15.00 & 1842 & 10000 & 92.1 & 407.9 \\
11 & 20.00 & 3070 & 100000 & 15.35 & 484.65 \\
12 & 30.00 & 6140 & 100000 & 30.7 & 469.3 \\
13 & 40.00 & 6160 & 100000 & 30.8 & 469.2 \\
14 & 50.00 & 6180 & 100000 & 30.9 & 469.1 \\
15 & 70.00 & 12350 & 100000 & 61.75 & 438.25 \\
16 & 90.00 & 12390 & 100000 & 61.95 & 438.05 \\
17 & 100.00 & 6260 & 100000 & 31.3 & 468.7 \\
18 & 130.00 & 18640 & 100000 & 93.2 & 406.8 \\
19 & 150.00 & 12510 & 100000 & 62.55 & 437.45 \\
20 & 200.00 & 31150 & 100000 & 155.75 & 344.25
\end{tabular}

\subsection{DNase I probing}

For DNase probing assays, 2 pmol of nucleic acid complexes were annealed and further incubated at room temperature for 5 min with buffer and metal ions for proper folding in $10 \mu \mathrm{l} .1 \mu \mathrm{l}$ DNase I buffer (10 mM Tris-HCl pH 7.6, $2.5 \mathrm{mM} \mathrm{MgCl}$, $0.5 \mathrm{mM} \mathrm{CaCl}_{2}$ ) and $1 \mu \mathrm{l}$ DNase I (RNase free, NEB) were added to the samples. Samples incubated at $37^{\circ} \mathrm{C}$ for $10 \mathrm{~min}$. The DNase I digestion stopped by addition of $3 \mu \mathrm{l}$ of $3 \mathrm{M} \mathrm{NaCl}$ and $100 \mu \mathrm{l}$ of ethanol and ethanol precipitation. Palettes were dried and dissolved in 3-5 $\mu \mathrm{H} \mathrm{H}_{2} \mathrm{O}$ plus 3-5 $\mu \mathrm{l}$ of loading dye (80\% formamide, 1xTBE, $50 \mathrm{mM}$ EDTA, $0.0025 \%$ each of bromophenol blue and xylene cyanol).

\subsection{DMS probing}

DMS was performed according to the reported procedure by Li et $\mathrm{al}^{39}$. For DMS probing 2 pmol of DNA/RNA complexes were annealed in 5-7 $\mu$ l. samples further incubated for $5 \mathrm{~min}$ at room temperature with reaction buffer and metal ions as stated for individual samples in final volume of $10 \mu \mathrm{l}$ for proper folding. $1 \mu \mathrm{l}$ of $2 \%$ dimethyl sulfate (DMS, from Riedel-de Haen) was added to the samples. Samples further incubated at room temperature for $10 \mathrm{~min}$. Methylation was stopped by addition of $8 \mu \mathrm{l}$ 
of DMS stop solution ( $1 \mathrm{M} ß$-mercaptoethanol and $1.5 \mathrm{M} \mathrm{NaCl}$ ) and further ethanol precipitation. The palettes were dissolved in $50 \mu \mathrm{l}$ of $10 \%$ piperidine and incubated at $90^{\circ} \mathrm{C}$ for $30 \mathrm{~min}$. samples were dried in speedvac $\left(35^{\circ} \mathrm{C}\right)$ and washed two times by addition of $100 \mu \mathrm{l} \mathrm{H}_{2} \mathrm{O}$ and subsequent vacuum drying in speedvac. Samples were dissolved in 3-5 $\mu \mathrm{l} \mathrm{H}_{2} \mathrm{O}$. Equal volume of loading dye was added to the samples prior to loading on a sequencing gel.

For DMS probing of active 6BX22 complex in the time course of reaction 5 samples were prepared by annealing of 3 pmol of radiolabeled trimmed 6BX22 in presence of $30 \mathrm{pmol}$ active substrates (R001 and Tr03). The annealing was performed in $7 \mu$ l. For one of the samples (control) only $\mathrm{H}_{2} \mathrm{O}$ was added to reach final $10 \mu \mathrm{l}$ volume. For the other four samples buffer and metal ions were added to reach standard 6BX22 reaction condition i.e. $50 \mathrm{mM}$ HEPES pH 7.5, $150 \mathrm{mM} \mathrm{NaCl}, 2 \mathrm{mM} \mathrm{KCl}$ and $20 \mathrm{mM} \mathrm{MnCl}_{2}$. At time points after addition of $\mathrm{Mn}^{2+}(0,5,20$ and $90 \mathrm{~min})$ one of the samples were taken from $37^{\circ} \mathrm{C}$ and subjected for DMS methylation at room temperature in presence of 2\% DMS. Methylation reactions were stopped after $10 \mathrm{~min}$ and piperidine cleavage was followed as explained.

\subsection{EMSA}

Binding assays have been performed in 5-10 $\mu \mathrm{l}$. Samples were incubated either at room temperature and electrophoresed at room temperature or incubated on ice and electrophoresed at cold room $\left(4^{\circ} \mathrm{C}\right)$. The gel plates used for casting the urea-free polyacrylamide gels for binding assays were $20 \mathrm{~cm}$ long, with $0.4 \mathrm{~mm}$ spacer, except it is mentioned otherwise $(8 \mathrm{~cm})$. Concentrated protein samples were provided by the group of Dr. Vladimir Pena, Macromolecular Crystallography, Max Planck Institute for Biophysical Chemistry, Göttingen, Germany. $20 \mu \mathrm{l}$ of $50 \mu \mathrm{M}$ protein stocks were prepared by dilution of proteins in protein dilution buffer. Aliquots were deep frozen in liquid nitrogen and kept at $-80^{\circ} \mathrm{C}$. Prior to binding assays, protein samples were thawed at room temperature for $10 \mathrm{~min}$ and kept on ice afterwards. The general buffers and solutions used in EMSA were prepared as follows.

\begin{tabular}{|c|c|}
\hline RNA dilution buffer & $50 \mathrm{mM}$ HEPES pH 7.5, $150 \mathrm{mM} \mathrm{NaCl}$ and $2 \mathrm{mM} \mathrm{KCl}$ \\
\hline Protein dilution buffer & $50 \mathrm{mM}$ HEPES pH 7.5, $150 \mathrm{mM} \mathrm{NaCl,} \mathrm{10 \%} \mathrm{v/v} \mathrm{glycerol} \mathrm{and} 2 \mathrm{mM}$ DTT \\
\hline $5 \mathrm{x}$ TB & $27 \mathrm{~g}$ Tris, $14 \mathrm{~g}$ boricacid, up to $500 \mathrm{ml} \mathrm{H}_{2} \mathrm{O}$ \\
\hline $8 \%$ gel solution $(0.5 x \mathrm{~TB})$ & $\begin{array}{l}3 \mathrm{ml} 40 \% \text { polyacrylamide, appropriate volume of } 3 \mathrm{M} \mathrm{MgCl}_{2}, 1.5 \mathrm{ml} 5 \mathrm{x} \text { TB, up to } 15 \\
\mathrm{ml} \mathrm{H}_{2} \mathrm{O}\end{array}$ \\
\hline Loading buffer & $\begin{array}{l}50 \% \text { Glycerol: } 10 \mathrm{x} \mathrm{Mg}^{2+} ; 2: 1 \text {. Loading buffer was prepared for each gel according to } \\
\text { final concentration of } \mathrm{Mg}^{2+} \text { in that special gel. }\end{array}$ \\
\hline
\end{tabular}

\subsubsection{Binding assay (strategy 1)}

In the first strategy samples were diluted, first and annealed afterwards. Here as an example the procedure for Fig 3.2-2 B is explained. The procedures for other gels in Fig 3.2-2 are similar with slight changes in the amount of the present RNAs and the order of addition of the RNAs. 20 pmol nonradiolabeled br3, 20 pmol U2 (R08) and 20 pmol of U6 snRNA were mixed in $5 \mu$ in presence of RNA 
dilution buffer. Samples were sequentially diluted with $2.5 \mu$ of RNA dilution buffer. At this stage, only $2.5 \mu$ is remained to each tube and samples are named $2 \mathrm{x}$ because the final volume for the binding assay will be increased to $5 \mu$ l. Table 5.20-1 shows an example of the final concentration of the dilution series.

Then, $1 \mu$ l of $1 \mathrm{nM}$ radiolabeled *br3 was added to each samples. Samples were annealed in thermocycler programmed for $2 \min 95^{\circ} \mathrm{C}$ and stepwise temperature decrease $\left(5^{\circ} \mathrm{C}\right.$ per min) down to $20^{\circ} \mathrm{C}$. To the $3.5 \mu \mathrm{l}$ annealed samples, $1.5 \mu \mathrm{l}$ of loading buffer was added to reach final volume of $5 \mu \mathrm{l}$. samples were loaded on $8 \%$ native gel containing $1 \mathrm{x} \mathrm{Mg}^{2+}$ (in this case: $2 \mathrm{mM} \mathrm{Mg}^{2+}$ ) and $0.5 \mathrm{x}$ TB.

Table 5.20-1 General preparation of serial dilutions for EMSA

\begin{tabular}{lllllll} 
& $\begin{array}{l}\text { RNA } \\
(\mathrm{pmol})\end{array}$ & $\begin{array}{l}\text { Starting } \\
\text { volume } \\
(\mu \mathrm{l})\end{array}$ & $\begin{array}{l}\text { [RNA] in } \\
2 \times \text { sample } \\
(\mu \mathrm{M})\end{array}$ & $\begin{array}{l}\text { remained } \\
\text { volume } \\
(\mu \mathrm{l})\end{array}$ & $\begin{array}{l}\text { RNA in 2x sample } \\
\text { after dilution } \\
(\mathrm{pmol})\end{array}$ & $\begin{array}{l}\text { Final [RNA] } \\
(\mu \mathrm{M})\end{array}$ \\
\hline 1 & 20 & 5 & 4 & 2.5 & 10 & 2 \\
2 & 10 & 5 & 2 & 2.5 & 5 & 1 \\
3 & 5 & 5 & 1 & 2.5 & 2.5 & 0.5 \\
4 & 2.5 & 5 & 0.5 & 2.5 & 1.25 & 0.25 \\
5 & 1.25 & 5 & 0.25 & 2.5 & 0.625 & 0.125 \\
6 & 0.625 & 5 & 0.125 & 2.5 & 0.3125 & 0.0625 \\
7 & 0.3125 & 5 & 0.0625 & 2.5 & 0.15625 & 0.03125 \\
8 & 0.15625 & 5 & 0.03125 & 2.5 & 0.078125 & 0.015625
\end{tabular}

\subsubsection{Binding assay (strategy 2)}

In the second strategy, samples were annealed at higher concentration and diluted afterwards with RNA dilution buffer. Additional RNA component or proteins were added to the dilute samples without further annealing. Here the procedure for Fig 3.2-4 A is explained. $10 \mathrm{nmol}$ of trace labeled *br3 and $10 \mathrm{nmol}$ of U2 RNA were annealed in presence of RNA dilution buffer in thermocycler programmed for 2 min $95^{\circ} \mathrm{C}$ and stepwise temperature decrease $\left(5^{\circ} \mathrm{C}\right.$ per min) down to $20^{\circ} \mathrm{C}$. Sample was diluted 100 -fold by RNA dilution buffer. The $0.01 \mu \mathrm{M}$ annealed oligonucleotide complexes were used for further annealing to the third RNA complex or protein or both. U6-sh snRNA and RNase H protein were diluted sequentially to the stated concentration, in RNA dilution buffer or protein dilution buffer. Binding assays were performed in $10 \mu \mathrm{l} .2 \mu \mathrm{l}$ of protein (desired concentration) or protein dilution buffer (control) and $1 \mu$ diluted U6-sh or only RNA dilution buffer (control), $1 \mu \mathrm{l} 10 \mathrm{x} \mathrm{Mg}^{2+}, 3 \mu \mathrm{l} 50 \%$ glycerol and $1 \mu \mathrm{l}$ RNA dilution buffer were added to $2 \mu \mathrm{l}$ of diluted annealed *br3 and U2. Samples were further incubated on ice for at least $1 \mathrm{~h}$.

Table 5.20-2 Final concentration of RNA and protein components in binding assays that is shown in Fig 3.2-4 A.

\begin{tabular}{|c|c|c|c|c|c|c|c|c|c|c|c|c|c|c|c|c|c|c|c|c|c|c|}
\hline & \multicolumn{6}{|c|}{$* b r 3+U 2+\operatorname{Pr}$} & \multicolumn{9}{|c|}{ *br3+U2+ U6-sh } & \multicolumn{6}{|c|}{${ }^{*} \mathrm{br} 3+\mathrm{U} 2+\mathrm{U} 6-\mathrm{sh}+\mathrm{Pr}$} & $S M \underset{\text { boil }}{S M}$ \\
\hline lane & 1 & 2 & 3 & 4 & 5 & 6 & 7 & 9 & 10 & 11 & 12 & 13 & 14 & 15 & 17 & 18 & 19 & 20 & 21 & 22 & 23 & 2526 \\
\hline final Rnase $\mathrm{H}(\mu \mathrm{M})$ & 0 & 0.02 & 0.1 & 0.2 & 1 & 2 & 10 & & & & - & & & & 0 & 0.02 & 0.1 & 0.2 & 1 & 2 & 10 & \\
\hline final U6-sh $(\mu \mathrm{M})$ & & & & - & & & & 0 & 0.00 & 0.01 & $0 ., 02$ & 0.1 & 0.2 & 1 & 0 & 0.002 & 0.01 & 0.02 & 0.1 & 0.2 & 1 & \\
\hline final *br3+U2 ( $\mu \mathrm{M})$ & & & & 0.002 & & & & & & & 0.002 & & & & & & & 0.002 & & & & 0.002 \\
\hline
\end{tabular}




\subsection{Ribosomal interference by branched RNAs}

HemK mut3 DNA template for in vitro transcription was produced by PCR from HemK plasmid, using primers which introduced the mutations. Preparation of the DNA template and subsequent transcription of HemK mut3 mRNA was performed by Riccardo Belardinelli, department of Physical Biochemistry at Max Planck Institute for Biophysical Chemistry, Göttingen. Adaptor RNAs were transcribed in vitro from single stranded DNA template using T7 promoter. Kinetic scale mRNA branch formation (1 pmol) followed standard kinetic conditions for 10DM24 $\mathrm{Tb}^{3+}$ mediated acceleration (2:10:20 acceptor: DNA: donor, pH 7.5, $80 \mathrm{mM} \mathrm{MgCl}_{2}$ and $100 \mu \mathrm{M} \mathrm{TbCl}_{3}$ in $10 \mu \mathrm{l}$ at $37^{\circ} \mathrm{C}$ ). Preparative scale (500 pmol) mRNA branch formation was performed by 10DM24 deoxyribozyme in $200 \mu \mathrm{l}$ for $20 \mathrm{~min}$ at $37^{\circ} \mathrm{C}$.

E. Coli 70S ribosome and aminoacylated tRNAs were purified in the department of Physical Biochemistry. IF1, IF2, IF3, EF-Tu and EF-G were recombinant and were provided from the same department. To form $70 \mathrm{~S}$ initiation complex (IC), ribosomal subunits $(0.1 \mu \mathrm{M})$, 3-fold excess of $\left[{ }^{3} \mathrm{H}\right]$ fMet-tRNA $^{\text {fMet }}$ (specific activity $3500 \mathrm{dpm} / \mathrm{pmol}$ ) and initiation factors IF1, IF2 and IF3 were incubated with mRNA $(0-0.5 \mu \mathrm{M})$ and GTP $(0.5 \mathrm{mM})$ in buffer TAKM7 (Tris- $\mathrm{HCl} \mathrm{pH} 7.5, \mathrm{NH}_{4} \mathrm{Cl} 70 \mathrm{mM}, \mathrm{KCl}$ $30 \mathrm{mM}, \mathrm{MgCl}_{2} 7 \mathrm{mM}$ ) for $20 \mathrm{~min}$ at $37^{\circ} \mathrm{C}$. The samples were passed through a nitrocellulose filter. Filters were washed with 3-4 $\mathrm{ml}$ of TAKM7 buffer, dried and their radioactivity was quantified in a scintillation cocktail. In the absence of mRNA no 70S IC can be formed. Free $\left[{ }^{3} \mathrm{H}\right]$ fMet-tRNA ${ }^{\text {fMet }}$ has lower molecular weight than the cut off of the filter and therefore passes through the filter. The amount of bound $\left[{ }^{3} \mathrm{H}\right]$ fMet-tRNA ${ }^{\text {fMet }}$ to filter in the absence of mRNA was measured as background. After background correction the amount of incorporated $\left[{ }^{3} \mathrm{H}\right]$ fMet-tRNA ${ }^{\text {fMet }}$ as a function of the ratio of mRNA over ribosomal subunits was plotted.

To form pre-translocation complex, 70S IC was formed with 70S ribosome $(0.3 \mu \mathrm{M})$, 3- fold excess of initiation factors and $\left[{ }^{3} \mathrm{H}\right]$ fMet-tRNA ${ }^{\mathrm{fMet}}(0.9 \mu \mathrm{M})$ and slight excess of mRNA and branched mRNAs $(0.4 \mu \mathrm{M})$. This specific concentration of mRNA was used to perform the experiment at concentrations which the system is saturated with mRNA. Data in Fig 3.3-3 showed system reaches saturation by 1:1 ratio of $70 \mathrm{~S}$ and mRNA. In parallel activated ternary complex was prepared by mixing EF-Tu $(1.2 \mu \mathrm{M})$ and GTP $(1 \mathrm{mM})$. The mixture incubated $15 \mathrm{~min}$ at $37^{\circ} \mathrm{C}$ with phosphoenol pyruvate (PEP, $\left.3 \mathrm{mM}\right)$ and pyruvate kinase (PK, 0.02 units) in presence of TAKM7 buffer to avoid presence of GDP and therefore non-active EF-Tu. $\left[{ }^{14} \mathrm{C}\right]$ Glu-tRNA ${ }^{\text {Glu }}(0.6 \mu \mathrm{M}$, specific activity $577 \mathrm{dpm} / \mathrm{pmol})$ was added to the mixture and incubated further 2 min to form the ternary complex. The 70S IC were mixed to the activated ternary complex 1:1 and incubated for further 1-2 min. The samples filtered through the nitrocellulose filters and incorporated radioactivity was measured. Ternary complex has molecular weight below the nitrocellulose cut off. And therefore, the bound $\left[{ }^{14} \mathrm{C}\right]$ Glu-tRNA ${ }^{\text {Glu }}$ to the nitrocellulose represents the bound $\left[{ }^{14} \mathrm{C}\right]$ Glu-tRNA ${ }^{\text {Glu }}$ to the $70 \mathrm{~S}$ ribosome. An aliquot of ternary 
complex in the absence of ribosomal subunits were filtered and the bound $\left[{ }^{14} \mathrm{C}\right]$ Glu-tRNA ${ }^{\text {Glu }}$ were considered as background.

For in vitro translation of HemK mRNA and its branched forms, 70S IC complexes were prepared with $70 \mathrm{~S}$ ribosome $(0.1 \mu \mathrm{M})$ and 3-fold excess of non-radiolabeled fMet-tRNA ${ }^{\text {fMet }}$ and initiation factors, as described earlier. mRNA concentration was $0.15 \mu \mathrm{M}$. Elongation complexes were prepared by mixing EF-Tu (60 $\mu \mathrm{M})$, EF-G (2 $\mu \mathrm{M}), \mathrm{MgCl}_{2}(2 \mathrm{mM})$, DTT (2 mM), GTP (1 mM), PEP (3 mM) and PK (0.02 units) in presence of TAKM7 buffer and 15 min incubation at $37^{\circ} \mathrm{C}$. Total tRNA $(30 \mu \mathrm{M})$ containing 1 $2 \mu \mathrm{M}$ radiolabeled aminoacylated tRNA were added to the mixture and incubated for further 2 min. Two different combination of radiolabeled aminoacylated tRNA were used. 1) $\left.{ }^{3} \mathrm{H}\right]$ Gly-tRNA ${ }^{\text {Gly }}$, $6000 \mathrm{dpm} / \mathrm{pmol}$ and $\left[{ }^{14} \mathrm{C}\right]$ Pro-tRNA $\left.{ }^{\text {Pro }}, 566 \mathrm{dpm} / \mathrm{pmol}, 2\right)\left[{ }^{3} \mathrm{H}\right]$ Phe-tRNA ${ }^{\text {Phe }}, 2480 \mathrm{dpm} / \mathrm{pmol}$ and $\left[{ }^{14} \mathrm{C}\right]$ Leu-tRNA $^{\text {Leu }}, 187 \mathrm{dpm} / \mathrm{pmol}$. 70S IC and elongation complexes were mixed 1:1 and incubated for further $10 \mathrm{~min}$ at $37^{\circ} \mathrm{C}$. Reactions were quenched with $0.5 \mathrm{M} \mathrm{NaOH}$ and, $30 \mathrm{~min}$ at $37^{\circ} \mathrm{C}$. $20 \mu \mathrm{l}$ of glacial acetic acid, $20 \mu \mathrm{lBSA} 10 \mu \mathrm{g} / \mu \mathrm{l}, 1200 \mu \mathrm{l}$ 10\% trichloroacetic acid (TCA, ice-cold) added to the samples for precipitation at $-20^{\circ} \mathrm{C}$ at least for $30 \mathrm{~min}$. samples were filter and washed with $5 \mathrm{ml} 6 \%$ TCA (ice-cold). The filters were dried and radioactivity was measured in scintillator. 


\section{Appendix}

\subsection{List of DNAs}

Enzymatic core of the deoxyribozymes are marked with bold letter. Mutations from the original sequences are marked with red color. For minimized versions of deoxyribozymes the nucleotides remaining from the mutable region (linker region) are marked with purple color. DNA overhangs which were only present to accommodate proper DNA length for PAGE separation are marked with gray color. For the 3HJ forming deoxyribozymes i.e. 7S11 and 10DM24, the region which forms the P4 stem with the acceptor RNA is underlined.

\begin{tabular}{|c|c|c|}
\hline DNA & description & sequence \\
\hline D001 & 6BX22, original binding arms & $\begin{array}{l}\text { CGAAGCGCTAGAACATCAGGGGGAGCGAGCACTAATACAAGCGGGTAGGAGGC } \\
\text { CCTAGTACATGAGACTT }\end{array}$ \\
\hline D002 & 6BX22, original core, for standard substrates & CAACGCTAGCACATCAGGGGGAGCGAGCACTAATACAAGCGGGTAGGAGGCCC \\
\hline D003 & $\begin{array}{l}\text { 6BX22, original core, for short donor substrate } \\
\text { and CoMA }\end{array}$ & $\begin{array}{l}\text { TAGTACATGAGAC } \\
\text { CAACAGCACATCAGGGGGAGCGAGCACTAATACAAGCGGGTAGGAGGCCCTAG } \\
\text { TACATGAGACTT }\end{array}$ \\
\hline D004 & 6BX22, rA mutant library & $\begin{array}{l}\text { CAACAGCACAT } x x x x x x x x x x x x x x x x x x x x x x x x x x x x x x x x x x x x x x x T A G \\
\text { TACATGAGACTT } x=r A / d N 5 / 95\end{array}$ \\
\hline D005 & 6BX22, rG mutant library & $\begin{array}{l}\text { CAACAGCACATXXXXXXXXXXX } X X X X X X X X X X X X X X X X X X X X X X X X X X X X X X X T A G \\
\text { TACATGAGACTT } X=r G / d N 5 / 95\end{array}$ \\
\hline D006 & 6BX22, rC mutant library & $\begin{array}{l}\text { CAACAGCACATXXXXXXXXXXX } \\
\text { TACATGAGACTT } \quad \mathrm{X}=\mathrm{rC} / \mathrm{dN} 5 / 95\end{array}$ \\
\hline D007 & 6BX22, rU mutant library & $\begin{array}{l}\text { CAACAGCACATXXXXXXXXXXXXXXXXX } \\
\text { TACATGAGACTT } X=r U / d N \quad 5 / 95\end{array}$ \\
\hline D008 & $6 \mathrm{BX} 22$, trim +1 & CAACGCTAGCACATCAGGGGGAGCGGCGGGTAGGAGGCCCTAGTACATGAGAC \\
\hline D009 & $6 \mathrm{BX} 22$, trim +8 & $\begin{array}{l}\text { CAACGCTAGCACATCAGGGGGAGCGAGCAAGCGGGTAGGAGGCCCTAGTACAT } \\
\text { GAGAC }\end{array}$ \\
\hline D010 & 6BX22, trim & $\begin{array}{l}\text { CAACGCTAGCACATCAGGGGGAGCTTGCGGGTAGGAGGCCCTAGTACATGAGA } \\
\text { C }\end{array}$ \\
\hline D011 & 6BX22, original core, for YBL059W & $\begin{array}{l}\text { GTTATTGCCTATGCATCAGGGGGAGCGAGCACTAATACAAGCGGGTAGGAGGC } \\
\text { CCTAGTAAACGTGTTCTTTG }\end{array}$ \\
\hline D012 & 6BX22, trimmed, for YBL059W & $\begin{array}{l}\text { GTTATTGCCTATGCATCAGGGGGAGCTTGCGGGTAGGAGGCCCTAGTAAACGT } \\
\text { GTTCTTTG }\end{array}$ \\
\hline D013 & 6BX22, trimmed, for branched RNA 7,8,9 & $\begin{array}{l}\text { CGAAGCGCTAGAACATCAGGGGGAGCTTGCGGGTAGGAGGCCCTAGTACATGA } \\
\text { GACTT }\end{array}$ \\
\hline D014 & 6BX22, original core, G6A & $\begin{array}{l}\text { CAACGCTAGCACATCAGGGAGAGCGAGCACTAATACAAGCGGGTAGGAGGCCC } \\
\text { TAGTACATGAGAC }\end{array}$ \\
\hline D015 & 6BX22, trimmed, G6A & $\begin{array}{l}\text { CAACGCTAGCACATCAGGGAGAGCTTGCGGGTAGGAGGCCCTAGTACATGAGA } \\
\text { C }\end{array}$ \\
\hline D016 & 6BX22, trimmed, G3A & $\begin{array}{l}\text { CAACGCTAGCACATCAAGGGGAGCTTGCGGGTAGGAGGCCCTAGTACATGAGA } \\
\text { C }\end{array}$ \\
\hline D017 & 6BX22, trimmed, G16A & $\begin{array}{l}\text { CAACGCTAGCACATCAGGGGGAGCTTGCGAGTAGGAGGCCCTAGTACATGAGA } \\
\text { C }\end{array}$ \\
\hline D018 & 6BX22, trimmed, G20A & $\begin{array}{l}\text { CAACGCTAGCACATCAGGGGGAGCTTGCGGGTAAGAGGCCCTAGTACATGAGA } \\
\text { C }\end{array}$ \\
\hline D019 & 6BX22, trimmed, G16C & $\begin{array}{l}\text { CAACGCTAGCACATCAGGGGGAGCTTGCGCGTAGGAGGCCCTAGTACATGAGA } \\
\text { C }\end{array}$ \\
\hline D020 & 6BX22, trimmed, G16T & $\begin{array}{l}\text { CAACGCTAGCACATCAGGGGGAGCTTGCGTGTAGGAGGCCCTAGTACATGAGA } \\
\text { C }\end{array}$ \\
\hline D021 & 6BX22, trimmed, G6C & $\begin{array}{l}\text { CAACGCTAGCACATCAGGGCGAGCTTGCGGGTAGGAGGCCCTAGTACATGAGA } \\
\text { C }\end{array}$ \\
\hline D022 & 6BX22, trimmed, G6T & $\begin{array}{l}\text { CAACGCTAGCACATCAGGGTGAGCTTGCGGGTAGGAGGCCCTAGTACATGAGA } \\
\text { C }\end{array}$ \\
\hline D023 & 6BX22, trimmed, dNAIM pool; dG r-7deaza G & $\begin{array}{l}\text { CAACGCTAGCACATCAXXXXXAXCTTXCXXXTAXXAXXCCCTAGTACATGAGA } \\
\text { C } \mathrm{x}=\mathrm{r}-7 \text { deasa } \mathrm{G} / \mathrm{dG} 5: 95\end{array}$ \\
\hline D024 & 6BX22, trimmed, dNAIM pool; dG rG & $\begin{array}{l}\text { CAACGCTAGCACATCAXXXXXAXCTTXCXXXTAXXAXXCCCTAGTACATGAGA } \\
\mathrm{C} X=\mathrm{rG} / \mathrm{dG} 5: 95\end{array}$ \\
\hline D025 & $\begin{array}{l}\text { 6BX22, trimmed, dNAIM pool; dG r- } \\
\text { Aminopurine }\end{array}$ & $\begin{array}{l}\text { CAACGCTAGCACATCAXXXXXAXCTTXCXXXTAXXAXXCCCTAGTACATGAGA } \\
\text { C } x=r \text {-Aminopurine/dG } 5: 95\end{array}$ \\
\hline D026 & 6BX22, trimmed, dNAIM pool; dG r-m1G & $\begin{array}{l}\text { CAACGCTAGCACATCAXXXXXAXCTTXCXXXTAXXAXXCCCTAGTACATGAGA } \\
\text { C } \mathrm{x}=\mathrm{r}-\mathrm{m} 1 \mathrm{G} / \mathrm{dG} 5: 95\end{array}$ \\
\hline D027 & T7 promoter & CTGTAATACGACTCACTATA \\
\hline
\end{tabular}




\begin{tabular}{|c|c|}
\hline D028 & template for Tr01 \\
\hline D029 & template for Tr02 \\
\hline D030 & template for Tr03 \\
\hline D031 & template for Tr04, YBL059W \\
\hline D032 & template for Tr05 \\
\hline D033 & template for Tr06 \\
\hline D034 & template for Tr07 \\
\hline D035 & template for yU6snRNA(30-84) \\
\hline D036 & $\begin{array}{l}\text { forward primer for U6 starting at nt } 30 \text { (includin } \\
\text { 20nt T7 promoter) }\end{array}$ \\
\hline D037 & reverse primer for U6 ending with 102 \\
\hline D038 & template for yU2-like fragment (binding to br3) \\
\hline D039 & reverse primer for U6 upto BamH1 \\
\hline D040 & 6BX22 minimized core \\
\hline D041 & D010 5'-binding arm complement to R05 \\
\hline D042 & $\begin{array}{l}\text { D010 3'-binding arm complement to R03 plus } \\
\text { two nt overhang }\end{array}$ \\
\hline D043 & splint for 3'-labeling of 6BX22 CoMA \\
\hline D044 & control DNA for terbium luminescence \\
\hline D045 & D010 3'-binding arm complement to R03 \\
\hline D046 & 9F7 original \\
\hline D047 & 9F7 short binding arm for CoMA \\
\hline D048 & 9F7 rA mutant library \\
\hline D049 & 9F7 rG mutant library \\
\hline D050 & 9F7 rC mutant library \\
\hline D051 & 9F7 rU mutant library \\
\hline D052 & 9F7 trim-3 \\
\hline D053 & 9F7 trim-2 \\
\hline D054 & 9F7 trim-1 \\
\hline D055 & 9F7 trim \\
\hline D056 & 9F7 trimmed with binding arm for "1G" donor \\
\hline D057 & 9F7 trimmed, blunt end for Tb lumin. \\
\hline D058 & 9F7 G4A, trimmed, blunt \\
\hline D059 & 9F7 G4C, trimmed blunt \\
\hline D060 & 9F7 G4T, trimmed blunt \\
\hline D061 & template for $\operatorname{Tr} 12$ \\
\hline D062 & template for $\operatorname{Tr} 13$ \\
\hline D063 & template for 9F7 "1G" donor \\
\hline D064 & $\begin{array}{l}\text { complementary to donor substrate for HPLC } \\
\text { analysis }\end{array}$ \\
\hline D065 & 9F13 original \\
\hline D066 & 9F13 short binding arm for CoMA \\
\hline D067 & 9F13 rA mutant library \\
\hline D068 & 9F13 rG mutant library \\
\hline D069 & 9F13 rC mutant library \\
\hline D070 & 9F13 rU mutant library \\
\hline D071 & 9F13 minus 11nt \\
\hline
\end{tabular}

TCCGCGCTAGAACATACTATAGTGAGTCGTATTACAGCGTGCGT

CCAGCACATACTATAGTGAGTCGTATTACAGCGTGCGT

CCGCTAGCACATACTATAGTGAGTCGTATTACAGCGTGCGT

CTATGTAAATAAGTTATGTTAGTAAACGTGTTCTTTGAGTATGAGGCCGAAGT TATTGCCTATGCATACTATAGTGAGTCGTATTACAGCGTGCGT CGATGTTAGTACATGAGACTTCCTATAGTGAGTCGTATTACAG

GAATGAAGCAATCGATGTTAGTACATGAGACTTCCTATAGTGAGTCGTATTAC AG

ATGGGATGCTGCAAGCGCTAGAACATACTATAGTGAGTCGTATTACAG

GTTCATCCTTATGCAGGGGAACTGCTGATCATCTCTGTATTGTTTCAAATTGA CCTATAGTGAGTCGTATTACAG

CTGTAATACGACTCACTATAGGTCAATTTGAAACAATACAG

AAATCTCTTTGTAAAACGGTTC

CACATACTACACTTGATCTAAGCCAAAAGGCAAAGAGATTCTATAGTGAGTCG TATTACAG

GTCCTTTAAAACGAAATAAATCTC

CAGGGGGAGCTTGCGGGTAGGAGGCCC

CAACGCTAGCACAT

TAGTACATGAGACGCAC

GTAAGTCTCATGTACTp - $3^{\prime}$

GAATGAAGCAATCGATGTTAGTACATGAGACTTCCTATAGTGAGTCG TATTACAG

TAGTACATGAGAC

CAACGTCGCCATCTCAATGAGGCTTGGCAGGGATTTAGTATTTTAACACTCCC GGGTAGTCGTATTA

CAACATCTCAATGAGGCTTGGCAGGGATTTAGTATTTTAACACTCCCGGGTAG TCGTATTA

СААСАТСТС $X X X X X X X X X X X X X X X X X X X X X X X X X X X X X X X X X X X X X X X X X X A G$ TCGTATTA $X=r A / d N \quad 5 / 95$

CААСАТСТС $X X X X X X X X X X X X X X X X X X X X X X X X X X X X X X X X X X X X X X X X X X A G$ TCGTATTA $X=r G / d N \quad 5 / 95$

CААСАТСТС $X X X X X X X X X X X X X X X X X X X X X X X X X X X X X X X X X X X X X X X X X X A G$

TCGTATTA $X=r C / d N \quad 5 / 95$

СААСАТТТ $X X X X X X X X X X X X X X X X X X X X X X X X X X X X X X X X X X X X X X X X X X A G$

TCGTATTA $X=r U / d N \quad 5 / 95$

CAACGTCGCCATCTCAATGAGGCTTGGCAGGGCCCGGGTAGTCGTATTA

CAACGTCGCCATCTCAATGAGGCTTGGCAGGGTCCCGGGTAGTCGTATTA

CAACGTCGCCATCTCAATGAGGCTTGGCAGGGCTCCCGGGTAGTCGTATTA

CAACGTCGCCATCTCAATGAGGCTTGGCAGGGACTCCCGGGTAGTCGTATTA

TGGTGTGGATGTTAATGAGGCTTGGCAGgGACTCCCGgGTAGTCGTATTA

CCGTCGCCATCTCAATGAGGCTTGGCAGGGACTCCCGGGTAGTCGTATTA

CCGTCGCCATCTCAATAAGGCTTGGCAGGGACTCCCGGGTAGTCGTATTA

CCGTCGCCATCTCAATCAGGCTTGGCAGGGACTCCCGGGTAGTCGTATTA

CCGTCGCCATCTCAATTAGGCTTGGCAGGGACTCCCGGGTAGTCGTATTA

CCGTCGCCATCTCTTGGTATAGTGAGTCGTATTACAG

CCATCTCTTGGTATAGTGAGTCGTATTACAG

TGGTGTGGATGTTATTCTATAGTGAGTCGTATTACAGT

CCGTCGCCATCTCTTCC

\author{
CAACGTCGCCATCTCAGGATGTGGGGTTTTGCCCGAGGGTATGGCAGTGGGGA \\ GAGAGTCGTATTA \\ CAACATCTCAGGATGTGGGGTTTTGCCCGAGGGTATGGCAGTGGGGAGAGAGT \\ CGTATTA \\ CААСАТСТС XXXXXXXXXXXXXXXXXXXXXXXXXXXX \\ CGTATTA $X=r A / d N \quad 5 / 95$ \\ СААСАТТС $X X X X X X X X X X X X X X X X X X X X X X X X X X X X X X X X X X X X X X X X$ AGT \\ CGTATTA $X=r G / d N \quad 5 / 95$ \\ СААСАТТТ $X X X X X X X X X X X X X X X X X X X X X X X X X X X X X X X X X X X X X X X X G G T$ \\ CGTATTA $X=r C / d N \quad 5 / 95$ \\ СААСАТСТС $X X X X X X X X X X X X X X X X X X X X X X X X X X X X X X X X X X X X X X X X G A T$ \\ CGTATTA $X=r U / d N \quad 5 / 95$ \\ CAACATCTCAGGATGTGGGGTTTTGCCCAGTGGGGAGAGAGTCGTATTA
}




\begin{tabular}{|c|c|}
\hline D072 & 9F13 minus $14 \mathrm{nt}$ \\
\hline D073 & 9F13 replace 14 nt by $1 x \mathrm{~T}$ \\
\hline D074 & 9F13 replace $14 \mathrm{nt}$ by $2 \mathrm{xT}$ \\
\hline D075 & 9F13 replace $14 \mathrm{nt}$ by $3 \mathrm{xT}$ \\
\hline D076 & 9F13 minus 3xT \\
\hline D077 & 9F13 combination \\
\hline D078 & 9F13 trimmied \\
\hline D079 & 6CE8 original enzyme \\
\hline D080 & template for $\operatorname{Tr} 15$ \\
\hline D081 & $7 \mathrm{~S} 11$ \\
\hline D082 & 10DM24 original \\
\hline D083 & 10DM24 for R05 G10C (R17) \\
\hline D084 & 10DM24 for R05 G10A (R18) \\
\hline D085 & 10DM24 for R05 G10A (R19) \\
\hline D086 & 10DM24 for R05 G10A (R20) \\
\hline D087 & 10DM24 for R05 G10A (R21) \\
\hline D088 & 10DM24 for R05 G10A (R22) \\
\hline D089 & 10DM24 for R05 double mutant UAU (R23) \\
\hline D090 & 10DM24 for R05 double mutant UAU (R24) \\
\hline D091 & 10DM24 for R05 double mutant UAU (R25) \\
\hline D092 & 10DM24 for R05 double mutant UAU (R26) \\
\hline D093 & 10DM24 for R05 double mutant UAU (R27) \\
\hline D094 & 10DM24 for R05 double mutant UAU (R28) \\
\hline D095 & 10DM24 for R05 double mutant UAU (R29) \\
\hline D096 & 10DM24 for R05 double mutant UAU (R30) \\
\hline D097 & 10DM24 for R05 double mutant UAU (R31) \\
\hline D098 & 10DM24 for A40 in yU6snRNA \\
\hline D099 & 10DM24 for A51 in yU6snRNA \\
\hline D100 & 10DM24 for A56 in yU6snRNA \\
\hline D101 & 10DM24 for A62 in yU6snRNA \\
\hline D102 & 10DM24 for A76 in yU6snRNA \\
\hline D103 & 10DM24 for A29 in RNA model R33 \\
\hline D104 & 10DM24 for A20 in RNA model R34 \\
\hline D105 & 10DM24 for A33 in RNA model R34 \\
\hline D106 & 10DM24 for A35 in RNA model R34 \\
\hline D107 & 10DM24 for A10 in Tr16 \\
\hline D108 & 10DM24 for A18 in Tr16 \\
\hline D109 & 10DM24 for A24 in Tr16 \\
\hline D110 & 10DM24 for A23 in $\operatorname{Tr} 17$ \\
\hline D111 & 10DM24 for A32 in Tr17 \\
\hline D112 & 10DM24 for A35 in Tr17 \\
\hline
\end{tabular}

CAACATCTCAGgATGTGgGgTTTTGCCCGgGgAGAGAGTCGTATTA CAACATCTCAGGATGTGGGGTTTTGCCCTGGgGAGAGAGTCGTATTA CAACATCTCAGGATGTGGGGTTTTGCCCTTGGGGAGAGAGTCGTATTA CAACATCTCAGGATGTGGgGTTTTGCCCTTTGgGGAGAGAGTCGTATTA CAACATCTCAGGATGTGGGGTGCCCGAGGGTATGGCAGTGGGGAGAGAGTCGT ATTA CAACATCTCAGGATGTGGgGTTGCCCTTTGgGGAGAGAGTCGTATTA CAACATCTCAGGATGTGGGGTGCCCTTGGGGAGAGAGTCGTATTA CAACGCGCTAGAACATGGCACTCAGAGCGCACGGCGAGTACATGAGACT CCGCGCTAGAACATTCTATAGTGAGTCGTATTACAGT CCGTCGCCATCTCCAGTGCAGGGCGTGAGGGCTCGGTTCCCGTATTATCC CCGTCGCCATCTCCCGTAGgTGAAGGGCGTGAGGGTTCCATTCCCGTATTATC $\mathrm{C}$ CCGTCGCCATCTCCCGTAGgTGAAGgGCGTGAGGGTTCCATTCCGGTATTATC $\mathrm{C}$ CCGTCGCCATCTCCCGTAGgTGAAGgGCGTGAGgGTTCCATTCCTGTATTATC CCGTCGCCATCTCCCGTAGGTGAAGGGCGTGAGGGTTCCATTCCAGTATTATC C CCGTCGCCATCTCCCGTAGGTGAAGGGCGTGACGGTTCCATTCCCGTATTATC $\mathrm{C}$ CCGTCGCCATCTCCCGTAGgTGAAGGGCGTGATGGTTCCATTCCCGTATTATC CCGTCGCCATCTCCCGTAGgTGAAGgGCGTGAAGGTTCCATTCCCGTATTATC CCGTCGCCATCTCCCGTAGGTGAAGGGCGTGAAGGTTCCATTCCAGTATTATC $\mathrm{C}$ CCGTCGCCATCTCCCGTAGGTGAAGGGCGTGATGGTTCCATTCCAGTATTATC C CCGTCGCCATCTCCCGTAGgTGAAGgGCGTGATGGTTCCATTCCTGTATTATC CCGTCGCCATCTCCCGTAGgTGAAGGGCGTGAAGGTTCCATTCCTGTATTATC CCGTCGCCATCTCCCGTAGGTGAAGGGCGTGACGGTTCCATTCCTGTATTATC $\mathrm{C}$ CCGTCGCCATCTCCCGTAGGTGAAGGGCGTGATGGTTCCATTCCGGTATTATC $\mathrm{C}$ CCGTCGCCATCTCCCGTAGgTGAAGgGCGTGAAGGTTCCATTCCGGTATTATC $\mathrm{C}$ CCGTCGCCATCTCCCGTAGgTGAAGgGCGTGACGgTTCCATTCCGGTATTATC CCGTCGCCATCTCCCGTAGGTGAAGGGCGTGACGGTTCCATTCCAGTATTATC C CCGTCGCCATCTCCCGTAGGTGAAGGGCTTGTTGGTTCCATTCCCAAATTGAC $\mathrm{C}$ CCGTCGCCATCTCCCGTAGgTGAAGGGCTCATCGGTTCCATTCCCTGTATTGT TTC

CCGTCGCCATCTCCCGTAGGTGAAGGGCGCTGAGGTTCCATTCCСАTCTCTGT ATTG

CCGTCGCCATCTCCCGTAGGTGAAGGGCGGAACGGTTCCATTCCGCTGATCAT CTC

CCGTCGCCATCTCCCGTAGGTGAAGGGCCATCCGGTTCCATTCCTATGCAGGG GAAC

CCGTCGCCATCTCCCGTAGGTGAAGGGCCGCATGGTTCCATTCCTGAAACGTG $\mathrm{GC}$

CCGTCGCCATCTCCCGTAGGTGAAGGGCTACGTGGTTCCATTCCTAGAAGAAG AC

CCGTCGCCATCTCCCGTAGGTGAAGGGCAGTTCGGTTCCATTCCTTAGCAATA CG

CCGTCGCCATCTCCCGTAGGTGAAGGGCTTAGTGGTTCCATTCCCTTTAGCAA TA

CCGTCGCCATCTCCCGTAGGTGAAGGGCCAAGCGGTTCCATTCCAGCTTTCC CCGTCGCCATCTCCCGTAGGTGAAGGGCCGAGAGGTTCCATTCCCCCAAAGCT CCGTCGCCATCTCCCGTAGGTGAAGGGCTAGTCGGTTCCATTCCCAGAGATCC CA

CCGTCGCCATCTCCCGTAGgTGAAGGGCAAGCCGGTTCCATTCCCCTTTAGTC CCGTCGCCATCTCCCGTAGGTGAAGGGCTGTCCGGTTCCATTCCTAAAAGCCT CC

CCGTCGCCATCTCCCGTAGGTGAAGGGCACGTGGGTTCCATTCCCCTTAAAAG $\mathrm{CC}$ 


\begin{tabular}{|c|c|}
\hline D113 & 10DM24 for A48 in $\operatorname{Tr} 17$ \\
\hline D114 & 10DM24 for A53 in $\operatorname{Tr} 17$ \\
\hline D115 & 10DM24 for A82 in HemK mRNA \\
\hline D116 & 10DM24 for A92 in HemK mRNA \\
\hline D117 & 10DM24 for A13 in R35 \\
\hline D118 & 10DM24 for A20 in R35 \\
\hline D119 & 10DM24 for A21 in R35 \\
\hline D120 & 10DM24 for A38 in SAM-III \\
\hline D121 & 10DM24 for A44 in SAM-III \\
\hline D122 & 10DM24 for A44 in SAM-III, for ATP \\
\hline D123 & 9DB1 for SAM-III (27|28) \\
\hline D124 & E5112 for cleavage of SAM-III at A20 \\
\hline D125 & E5112 for cleavage of SAM-III at A44 \\
\hline D126 & 10-23 for cleavage of SAM-III at G36 \\
\hline D127 & 8-17 U6A51 Cl-I \\
\hline D128 & 8-17 U6A76 Cl-II \\
\hline D129 & 10-23 U6A35 An-i \\
\hline D130 & 8-17 U6A62 An-ii \\
\hline D131 & 8-17 U6A97 An-iii \\
\hline D132 & DNA helper for NTP attachment \\
\hline D133 & template for $\operatorname{Tr} 16$ \\
\hline D134 & template for $\operatorname{Tr} 17$ \\
\hline D135 & template for SAM-III (28-53) \\
\hline D136 & $\begin{array}{l}\text { Disruptor } 1 \text { (to be used for labeling at position } \\
20 \text {, complementary to 32-53 of SAM-III ligated }\end{array}$ \\
\hline D137 & $\begin{array}{l}\text { Disruptor } 2 \text { (to be used for labeling at position } \\
44 \text {, complementary to } 1-22 \text { of SAM-III ligated } \\
\text { primer for U6 }\end{array}$ \\
\hline D139 & template for generic adaptor-1 \\
\hline D140 & template for generic adaptor-2 \\
\hline D141 & disruptor for 3'-end of HemK14 mRNA \\
\hline
\end{tabular}

CCGTCGCCATCTCCCGTAGGTGAAGGGCTCGACGGTTCCATTCCCAATATTAC GT CCGTCGCCATCTCCCGTAGGTGAAGGGCCACGGGGTTCCATTCCCGACTCAAT ATT GTCGGGTCGGGTCTCCCGTAGGTGAAGGGCAGTGTGGTTCCATTCCGATATTC CATAT GTCGGGTCGGGTCTCCCGTAGGTGAAGGGCTCGCGGGTTCCATTCCACCAGTG TTGAT CCGTCGCCATCTCCCGTAGGTGAAGGGCCACCAGGTTCCATTCCCCTTTCGGG AAC CCGTCGCCATCTCCCGTAGGTGAAGGGCGTGATGGTTCCATTCCCCACCATCC TT CCGTCGCCATCTCCCGTAGGTGAAGGGCGGTGAGGTTCCATTCCTCCACCATC CCGTCGCCATCTCCCGTAGGTGAAGGGCGGTGAGGTTCCATTCCTCCACCATC C CCGTCGCCATCTCCCGTAGgTGAAGgGCGGTGAGGTTCCATTCCTCCACCATC AACAACAACCGTCGCCATCTCCCGTAGgTGAAGGGCCCCCTGGTTCCATTCTT CGGTTACAAGGCA

TCGGTTACAAGGCATGGATCATACGGTCGGAGGGGTTTGCCGTTTAGGTGATT CCACCATCCTTTCGG

CATCTGGTGAGTCAGCTGACTCGAACCACCATCCTT

ATTCCCCCGTCAGCTGACTCGAATCGGTTACAAGG

AACCCCCTTTCGGTTAGgCTAGCTACAACGAAAGGCATCTGGTGA

GCTGATCATTCCGAGCCGgACGACTGTATTGTTTCAAATTGACACACACACAC ACA

ACACACACACACACGTAAAACGGTTCATCTCCGAGCCGGACGATATGCAGGGG AAC

TATTGTTTCAAAGGCTAGCTACAACGATGACCAAATGTCCACACACACACACA CAC

TCATCCTTATGCAGGGGAATCCGAGCCGgACGAGCTGATCATCTCACACACAC ACA

AAAACGAAATAAATTCCGAGCCGGACGACTTTGTAAAACGGACACACACACAC ACA GAAGAGATGGCGACGG

ACACGGTCGACTCAATATTACGTGTCCTTAAAAGCCTCCTTTAGTCTATAGTG AGTCGTATTACAG

TAGTCTCGAGATCCCAAGCTAGCTTTCCTATAGTGAGTCGTATTACAG ATTCCCCСTTTCGGTTACAAGGCATCTATAGTGAGTCGTATTACAG ATTCCCCCTTTCGGTTACAAG

ATTCCACCATCCTTTCGGGAAC

GTCCTTTAAAACGAAATAAATCTC GTCGGGTCGGGTCTCTTCCTATAGTGAGTCGTATTACAGCGT TGCTGCTGCTGCTTGTCGGGTCGGGTCTCTTCCTATAGTGAGTCGTATTACAG CGT AAGTTGGCTAAATG 


\subsection{List of synthetic RNAs}

\begin{tabular}{|c|c|c|}
\hline RNA & Description & sequence \\
\hline R01 & 6BX22 acceptor substrate & AAGUCUCAUGUACUAACAUCGAUUG \\
\hline R02 & 6BX22 acceptor substrate with no overhang & GUCUCAUGUACUAA \\
\hline R03 & 6BX22 acceptor substrate $\mathrm{C}$ overhang & GUCUCAUGUACUAAC \\
\hline R04 & 6BX22 acceptor substrate CAU overhang & GUCUCAUGUACUAACAU \\
\hline R05 & 6BX22 inactive donor substrate & GUAUGUGCUAGCGG \\
\hline R06 & 6BX22 substrate 5'-A mutant & AUAUGUGCUAGCGG \\
\hline R07 & 6BX22 acceptor substrate & GGAAGUCUCAUGUACUAACA \\
\hline R08 & part of U2snRNA & UGCUUAGAUCAAGUGUAGUAUGUGAGA \\
\hline R09 & 6BX22 acceptor substrate & UCUCAUGUACUAACA \\
\hline R10 & RNA for complex formation with yU6snRNA & GUCUGUAU \\
\hline R11 & RNA for complex formation with yU6snRNA & UGCUUAGAUCUAGUAGUCUGUAU \\
\hline R12 & acceptor substrate for 9F7(A14) and 9F13 (U13) & UAAUACGACUCACUAUA \\
\hline R13 & R012 A14dA & UAAUACGACUCACU (dA)UA \\
\hline R14 & 5'-delta Tr015, RNA helper for NTP attachment & GAAGAGAUGGCGACGG \\
\hline R15 & Tr012 analogue & GGAAGAGAUGGCGACGG \\
\hline R16 & 10DM24 acceptor RNA & GGAUAAUACGACUCAC \\
\hline R17 & R016 analog G10C & GGAUAAUACCACUCAC \\
\hline R18 & R016 analog G10A & GGAUAAUACAACUCAC \\
\hline R19 & R016 analog G10U & GGAUAAUACUACUCAC \\
\hline $\mathbf{R} 20$ & R016 analog C12G & GGAUAAUACGAGUCAC \\
\hline R21 & R016analog C12A & GGAUAAUACGAAUCAC \\
\hline R22 & R016 analog C12U & GGAUAAUACGAUUCAC \\
\hline R23 & R016 analog double mutant G10UC12U & GGAUAAUACUAUUCAC \\
\hline R24 & R016 analog double mutant G10UC12A & GGAUAAUACUAAUCAC \\
\hline R25 & R016 analog double mutant G10AC12A & GGAUAAUACAAAUCAC \\
\hline R26 & R016 analog double mutant G10AC12U & GGAUAAUACAAUUCAC \\
\hline R27 & R016 analog double mutant G10A/C12G & GGAUAAUACAAGUCAC \\
\hline R28 & R016 analog double mutant G10C/C12A & GGAUAAUACCAAUCAC \\
\hline R29 & R016 analog double mutant G10C/C12U & GGAUAAUACCAUUCAC \\
\hline R30 & R016 analog double mutant G10C/C12G & GGAUAAUACCAGUCAC \\
\hline R31 & R016 analog double mutant G10U/C12G & GGAUAAUACUAGUCAC \\
\hline R32 & RNA model for GTP ligation-1 & GGCCACGUUUCAAAUGCGUGCU \\
\hline R33 & RNA model for GTP ligation-2 & GUCUUCUUCUAAACGUAUUGCUAAAGAACUAA \\
\hline R34 & 5'part of SAM-III (1-27), for 9DB1 ligation, GAAU loop & GUUCCCGAAAGGAUGGUGGAAUCACCA \\
\hline R35 & 6CE8, acceptor substrate & AGUCUCAUGUACUAUCG \\
\hline
\end{tabular}




\subsection{List of transcribed RNAs}

Transcript Description

Tr01

Tr02

Tr03

Tr04

Tr05

Tr06

Tr07

Tr08

Tr09

Tr10

Tr11

Tr12

Tr13

Tr14

Tr15

Tr16

Tr17

Tr18

Tr19

Tr20

Tr21 thesis) YBL059W
6BX22 donor original

6BX22 donor short

6BX22 donor (standard donor in this

6BX22 substrate for lariat formation:

$$
\text { 6BX22 acceptor for br7 }
$$

6BX22 acceptor for br8

6BX22 donor for br9

yeast U6snRNA (30-84)

yeast U6snRNA (1-102)

yeast U6snRNA upto BamH1

yeast U2-like RNA, partly complement with br3

9F7,9F13,10DM24 donor original

9F7,9F13,10DM24 donor short

$9 F 7$ donor "1G"

6CE8 donor substrate

RNA model for GTP attachment-1

RNA model for GTP attachment-2

SAM-III (28-53)

HemK mRNA up to BamHI site

adapter 1- for ribosomal interference

adapter 2- for ribosomal interference sequence

GUAUGUGCUAGCGG

GUAUGUGCUGG

GUAUGUUCUAGCGCGGA

GUAUGCAUAGGCAAUAACUUCGGCCUCAUACUCAAAGAACACGUUUACUAACAUAACUUAUUUACA UAG

GGAAGUCUCAUGUACUAACAUCG

GGAAGUCUCAUGUACUAACAUCGAUUGCUUCAUUUC

GUAUGUUCUAGCGCUUGCAGCAUCCCAU

GGUCAAUUUGAAACAAUACAGAGAUGAUCAGCAGUUCCCCUGCAUAAGGAUGAAC

GGUUCGCGAAGUAACCCUUCGUGGACAUUUGGUCAAUUUGAAACAAUACAGAGAUGAUCAGCAGUU CCCCUGCAUAAGGAUGAACCGUUUUACAAAGAGAUUU

GGUUCGCGAAGUAACCCUUCGUGGACAUUUGGUCAAUUUGAAACAAUACAGAGAUGAUCAGCAGUU

CCCCUGCAUAAGGAUGAACCGUUUUACAAAGAGAUUUAUUUCGUUUUAAAGGATC

GAAUCUCUUUGCCUUUUGGCUUAGAUCAAGUGUAGUAUGUG

GGAAGAGAUGGCGACGG

GGAAGAGAUGG

GAAUAACAUCCACACCA

GAAUGUUCUAGCGCGG

GGAAAGUAGCUUGGGAUCUCGAGACUA

GACUAAAGGAGGCUUUUAAGGACACGUAAUAUUGAGUCGACCGUGU

GAUGCCUUGUAACCGAAAGGGGGAAU

GGUUCGCGAAGUAACCCUUCGUGGACAUUUGGUCAAUUUGAAACAAUACAGAGAUGAUCAGCAGUU CCCCUGCAUAAGGAUGAACCGUUUUACAAAGAGAUUUAUUUCGUUUUAAAGGATC

GGAAGAGACCCGACCCGAC

GGAAGAGACCCGACCCGACAAGCAGCAGCAGCA 


\subsection{List of branched products (preparative scale)}

\begin{tabular}{|c|c|c|c|c|}
\hline $\begin{array}{l}\text { branched } \\
\text { products }\end{array}$ & acceptor & donor & enzyme & \\
\hline br1 & R12 & $\operatorname{Tr} 12$ & 9F7 & $\begin{array}{l}\text { brP used for 9F7 DMS and DNase probing } \\
\text { brP used for 6BX22 DMS and DNase probing and CD analysis and also used for splicing }\end{array}$ \\
\hline br2 & R01 & Tr03 & 6BX22 & assays \\
\hline br3 & R07 & Tr01 & 6BX22 & brP used for splicing assays in collaboration with department of Cellular Biochemistry \\
\hline br4 & R01 & Tr01 & 6BX22 & brP used for splicing assays in collaboration with department of Cellular Biochemistry \\
\hline br5 & R01 & Tr02 & 6BX22 & brP used for splicing assays in collaboration with department of Cellular Biochemistry \\
\hline br6 & R04 & Tr02 & 6BX22 & brP used for splicing assays in collaboration with department of Cellular Biochemistry \\
\hline br7 & Tr05 & Tr01 & 6BX22 & brP used for splicing assays in collaboration with department of Cellular Biochemistry \\
\hline br8 & Tr06 & Tr01 & 6BX22 & brP used for splicing assays in collaboration with department of Cellular Biochemistry \\
\hline br9 & R01 & Tr07 & 6BX22 & brP used for splicing assays in collaboration with department of Cellular Biochemistry \\
\hline br10 & R07 & Tr03 & 6BX22 & brP used for binding assays \\
\hline br11 & Tr19 & Tr20 & 10DM24 & brP used for ribosomal interference \\
\hline br12 & Tr19 & Tr21 & 10DM24 & brP used for ribosomal interference \\
\hline
\end{tabular}




\subsection{Abbreviations}

\begin{tabular}{|c|c|}
\hline $2 A P$ & 2-aminopurine \\
\hline $3 \mathrm{HC}$ & 2-thienyl-3-hydroxychromone \\
\hline $3 \mathrm{HJ}$ & Three helix junction \\
\hline $6 \mathrm{Ml}$ & 6-methylisoxanthopterin \\
\hline $70 \mathrm{SIC}$ & $70 S$ initiation complex \\
\hline A & adenine \\
\hline$\AA$ & angstrom \\
\hline aatRNA & aminoacylated tRNA \\
\hline ABTS & 2,2'-azino-bis(3-ethylbenzothiazoline-6-sulfonic acid) \\
\hline APS & ammonium persulfate \\
\hline ATP & adenosine triphosphate \\
\hline AuNPT & gold nanoparticles \\
\hline AUR & Amplex UltraRed \\
\hline BP & branch point \\
\hline bp & base pair \\
\hline brP & branched product \\
\hline BSA & Bovine serum albumin \\
\hline BTT & 5-Benzylthio-1H-tetrazole \\
\hline C & cytosine \\
\hline$c^{7} G$ & 7-deazaguanine \\
\hline$C D$ & Circular Dichroism \\
\hline CHES & $\mathrm{N}$-Cyclohexyl-2-aminoethanesulfonic acid \\
\hline $\mathrm{Ci}$ & Curie; $1 \mathrm{Ci}=37$ Gigabecquerel \\
\hline CIAP & Calf intestinal alkaline phosphatase \\
\hline CoMA & combinatorial mutation interference analysis \\
\hline CTP & cytidine triphosphate \\
\hline Cy & cyanine \\
\hline dATP & deoxyadenosine triphosphate \\
\hline dCTP & deoxycytidine triphosphate \\
\hline dGTP & deoxyguanosine triphosphate \\
\hline DMAP & 4-Dimethylamino-pyridin \\
\hline DMS & dimethylsulfate \\
\hline DMT & $5^{\prime}-0-4,4^{\prime}$-dimethoxytrityl \\
\hline $\mathrm{dN}$ & deoxyribonucleotide \\
\hline DNA & deoxyribonucleic acid \\
\hline dNAIM & DNA-nucleotide analogue interference mapping \\
\hline$D^{t}$ & Trimmed DNA \\
\hline DTP & 2,6-diaminopurine ribonucleoside triphosphate \\
\hline DTS & DNA-templated synthesis \\
\hline DTT & dithiothreitol \\
\hline dTTP & deoxythymidine triphosphate \\
\hline E.Coli & Escherichia coli \\
\hline EDTA & Ethylenediaminetetraacetic acid \\
\hline EF-G & elongation factor-G \\
\hline EF-Tu & elongation factor thermo unstable \\
\hline ELISA & enzyme-linked immunosorbent assay \\
\hline EPR & Electron Paramagnetic Resonance \\
\hline ESIPT & Excited-state intramolecular proton transfer \\
\hline FAM & 5-carboxyfluorescein \\
\hline FRET & Förster resonance energy transfer \\
\hline G & guanine \\
\hline GlmS & glucosamine-6-phosphate activated riboswitch \\
\hline GTP & guanosine triphosphate \\
\hline h & hour \\
\hline HEPES & 4-(2-Hydroxyethyl)piperazine-1-ethanesulfonic acid \\
\hline HPLC & High-performance liquid chromatography \\
\hline HRP & hores raddish peroxidase \\
\hline
\end{tabular}




\begin{tabular}{|c|c|}
\hline IF & initiation factor \\
\hline is & inactivated substrates \\
\hline ISL & internal stem loop \\
\hline IVS & intervening sequence \\
\hline L-arm & left binding arm \\
\hline $\mathrm{m}^{1} \mathrm{G}$ & 1-methyl guanosine \\
\hline MANT-GTP & 2'- / 3'- O- (N'- Methylanthraniloyl)guanosine- 5'- O- triphosphate \\
\hline $\mathrm{MB}$ & methylene blue \\
\hline $\min$ & minute \\
\hline MMLV-RT & moloney mouse leukemia virus reverse transcriptase \\
\hline mRNA & messenger RNA \\
\hline $\mathrm{ms}$ & millisecond \\
\hline MS & mass spectroscopy \\
\hline $\mathrm{nm}$ & nanometer \\
\hline nt & nucleotide \\
\hline NTP & nucleoside triphosphate \\
\hline PAGE & polyacrylamide gel electrophoresis \\
\hline PCR & polymerase chain reaction \\
\hline PEG & Polyethylene glycol \\
\hline PELDOR & pulsed electron-electron double resonance \\
\hline PK & pyruvate kinase \\
\hline PNK & polynucleotide 5'-hydroxyl-kinase \\
\hline PS & phosphorothioate \\
\hline R-arm & right binding arm of a deoxyribozyme \\
\hline RCA & rolling circle amplification \\
\hline RF & release factors \\
\hline $\mathrm{rN}$ & ribonucleotide \\
\hline RNA & ribonucleic acid \\
\hline RRF & ribosomal release factor \\
\hline RRM & RNA recognition motif domain \\
\hline rRNA & Ribosomal RNA \\
\hline S.cer. & Saccharomyces cerevisiae \\
\hline SAM & S-adenosyl- methionin \\
\hline SD & Shine-Dalgarno \\
\hline snNRA & small nuclear ribonucleic acid \\
\hline SS & splice site \\
\hline B-gal & ß-galactosidase \\
\hline $\mathrm{T}$ & thymine \\
\hline TAMRA & 6-carboxytetramethylrhodamine \\
\hline TBAF & Tetrabutylamonium fluoride \\
\hline TBE & Tris Borate EDTA \\
\hline TCA & Tricloroacetic acid \\
\hline TEMED & Tetramethylethylenediamine \\
\hline $\mathrm{T}_{\mathrm{m}}$ & melting temperature \\
\hline TMB & $3,3^{\prime}, 5,5^{\prime}$-tetramethylbenzidine sulfate \\
\hline TMPyP4 & meso-5,10,15,20-Tetrakis-(N-methyl-4-pyridyl)porphine \\
\hline TOM & [(triisopropylsilyl)oxy]methyl \\
\hline TPP & Thiamine pyrophosphate \\
\hline TPP & thiamin pyrophosphate \\
\hline trim & trimmed \\
\hline TTP & thymidine triphosphate \\
\hline tRNA & transfer RNA \\
\hline U & uracil \\
\hline UTP & uridine triphosphate \\
\hline UV & ultra violet \\
\hline W.C. & Watson-Crick base pairing \\
\hline $\mathrm{ZnF}$ & Zinc Finger \\
\hline
\end{tabular}




\section{References}

1. Cech, T. R.; Zaug, A. J.; Grabowski, P. J., In vitro splicing of the ribosomal RNA precursor of Tetrahymena: involvement of a guanosine nucleotide in the excision of the intervening sequence. Cell 1981, 27 (3 Pt 2), 487-96.

2. Guerrier-Takada, C.; Altman, S., Catalytic activity of an RNA molecule prepared by transcription in vitro. Science (New York, N.Y.) 1984, 223 (4633), 285-6.

3. Cochrane, J. C.; Strobel, S. A., Catalytic strategies of self-cleaving ribozymes. Accounts of chemical research 2008, 41 (8), 1027-35.

4. Hammann, C.; Luptak, A.; Perreault, J.; de la Pena, M., The ubiquitous hammerhead ribozyme. RNA (New York, N.Y.) 2012, 18 (5), 871-85.

5. Walter, N. G.; Burke, J. M., The hairpin ribozyme: structure, assembly and catalysis. Current opinion in chemical biology 1998, 2 (1), 24-30.

6. Lambowitz, A. M.; Zimmerly, S., Group II introns: mobile ribozymes that invade DNA. Cold Spring Harbor perspectives in biology 2011, 3 (8), a003616.

7. Anderson, M.; Schultz, E. P.; Martick, M.; Scott, W. G., Active-Site Monovalent Cations Revealed in a 1.55-Å-Resolution Hammerhead Ribozyme Structure. Journal of molecular biology (0).

8. Tuerk, C.; Gold, L., Systematic evolution of ligands by exponential enrichment: RNA ligands to bacteriophage T4 DNA polymerase. Science (New York, N.Y.) 1990, 249 (4968), 505-10.

9. Robertson, D. L.; Joyce, G. F., Selection in vitro of an RNA enzyme that specifically cleaves singlestranded DNA. Nature 1990, 344 (6265), 467-8.

10.Breaker, R. R.; Joyce, G. F., A DNA enzyme that cleaves RNA. Chemistry \& biology 1994, 1 (4), 223-9. 11.Silverman, S. K., Deoxyribozymes: selection design and serendipity in the development of DNA catalysts. Accounts of chemical research 2009, 42 (10), 1521-31.

12.Santoro, S. W.; Joyce, G. F., A general purpose RNA-cleaving DNA enzyme. Proc Natl Acad Sci U S A 1997, 94 (9), 4262-6.

13.Wang, Y.; Silverman, S. K., Directing the outcome of deoxyribozyme selections to favor native $3^{\prime}-5^{\prime}$ RNA ligation. Biochemistry 2005, 44 (8), 3017-23.

14.Silverman, S. K., DNA as a versatile chemical component for catalysis, encoding, and stereocontrol. Angewandte Chemie (International ed. in English) 2010, 49 (40), 7180-201.

15.Schlosser, K.; Li, Y., Biologically inspired synthetic enzymes made from DNA. Chemistry \& biology 2009, $16(3), 311-22$.

16.Chandrasekar, J.; Silverman, S. K., Catalytic DNA with phosphatase activity. Proc Natl Acad Sci U S A 2013.

17.Wong, O. Y.; Pradeepkumar, P. I.; Silverman, S. K., DNA-catalyzed covalent modification of amino acid side chains in tethered and free peptide substrates. Biochemistry 2011, 50 (21), 4741-9.

18.Elbaz, J.; Lioubashevski, O.; Wang, F.; Remacle, F.; Levine, R. D.; Willner, I., DNA computing circuits using libraries of DNAzyme subunits. Nature nanotechnology 2010, 5 (6), 417-22.

19.Cairns, M. J.; King, A.; Sun, L. Q., Optimisation of the 10-23 DNAzyme-substrate pairing interactions enhanced RNA cleavage activity at purine-cytosine target sites. Nucleic acids research 2003, 31 (11), 2883-9.

20.Schlosser, K.; Gu, J.; Sule, L.; Li, Y., Sequence-function relationships provide new insight into the cleavage site selectivity of the 8-17 RNA-cleaving deoxyribozyme. Nucleic acids research 2008, 36 (5), 1472-81.

21.Cruz, R. P.; Withers, J. B.; Li, Y., Dinucleotide junction cleavage versatility of 8-17 deoxyribozyme. Chemistry \& biology 2004, 11 (1), 57-67.

22.Parker, D. J.; Xiao, Y.; Aguilar, J. M.; Silverman, S. K., DNA catalysis of a normally disfavored RNA hydrolysis reaction. J Am Chem Soc 2013, 135 (23), 8472-5. 
23.Coppins, R. L.; Silverman, S. K., A DNA enzyme that mimics the first step of RNA splicing. Nature structural \& molecular biology 2004, 11 (3), 270-4.

24.Wang, Y.; Silverman, S. K., Deoxyribozymes That Synthesize Branched and Lariat RNA. Journal of the American Chemical Society 2003, 125 (23), 6880-6881.

25.Wang, Y.; Silverman, S. K., Characterization of Deoxyribozymes That Synthesize Branched RNAt. Biochemistry 2003, 42 (51), 15252-15263.

26.Wang, Y.; Silverman, S. K., Efficient one-step synthesis of biologically related lariat RNAs by a deoxyribozyme. Angew. Chem. Int. Ed. 2005, 44 (36), 5863-6.

27.Pratico, E. D.; Wang, Y.; Silverman, S. K., A deoxyribozyme that synthesizes 2',5'-branched RNA with any branch-site nucleotide. Nucleic acids research 2005, 33 (11), 3503-12.

28.Zelin, E.; Wang, Y.; Silverman, S. K., Adenosine is inherently favored as the branch-site RNA nucleotide in a structural context that resembles natural RNA splicing. Biochemistry 2006, 45 (9), 276771.

29.Coppins, R. L.; Silverman, S. K., A Deoxyribozyme that Forms a Three-Helix-Junction Complex with its RNA Substrates and has General RNA Branch-Forming Activity. Journal of the American Chemical Society 2005, 127 (9), 2900-2907.

30.Coppins, R. L.; Silverman, S. K., Rational modification of a selection strategy leads to deoxyribozymes that create native 3'-5' RNA linkages. Journal of the American Chemical Society 2004, 126 (50), 16426-32.

31.Purtha, W. E.; Coppins, R. L.; Smalley, M. K.; Silverman, S. K., General deoxyribozyme-catalyzed synthesis of native 3'-5' RNA linkages. Journal of the American Chemical Society 2005, 127 (38), 131245.

32.Chandra, M.; Sachdeva, A.; Silverman, S. K., DNA-catalyzed sequence-specific hydrolysis of DNA. Nature chemical biology 2009, 5 (10), 718-20.

33.Xiao, Y.; Chandra, M.; Silverman, S. K., Functional compromises among pH tolerance, site specificity, and sequence tolerance for a DNA-hydrolyzing deoxyribozyme. Biochemistry 2010, 49 (44), 9630-7.

34.Xiao, Y.; Wehrmann, R. J.; Ibrahim, N. A.; Silverman, S. K., Establishing broad generality of DNA catalysts for site-specific hydrolysis of single-stranded DNA. Nucleic acids research 2012, 40 (4), 177886.

35.Gu, H.; Furukawa, K.; Weinberg, Z.; Berenson, D. F.; Breaker, R. R., Small, Highly Active DNAs That Hydrolyze DNA. Journal of the American Chemical Society 2013, 135 (24), 9121-9129.

36.Dokukin, V.; Silverman, S. K., Lanthanide ions as required cofactors for DNA catalysts. Chemical science (Royal Society of Chemistry : 2010) 2012, 3 (5), 1707-1714.

37.Cuenoud, B.; Szostak, J. W., A DNA metalloenzyme with DNA ligase activity. Nature 1995, 375 (6532), 611-614.

38.Li, Y.; Breaker, R. R., Phosphorylating DNA with DNA. Proceedings of the National Academy of Sciences 1999, 96 (6), 2746-2751.

39.Li, Y.; Liu, Y.; Breaker, R. R., Capping DNA with DNA†. Biochemistry 2000, 39 (11), 3106-3114.

40.Sreedhara, A.; Li, Y.; Breaker, R. R., Ligating DNA with DNA. Journal of the American Chemical Society 2004, 126 (11), 3454-60.

41.Silverman, S. K., Catalytic DNA (deoxyribozymes) for synthetic applications-current abilities and future prospects. Chemical Communications 2008, 0 (30), 3467-3485.

42.Lee, C. S.; Mui, T. P.; Silverman, S. K., Improved deoxyribozymes for synthesis of covalently branched DNA and RNA. Nucleic acids research 2011, 39 (1), 269-79.

43.Pradeepkumar, P. I.; Hobartner, C.; Baum, D. A.; Silverman, S. K., DNA-catalyzed formation of nucleopeptide linkages. Angewandte Chemie (International ed. in English) 2008, 47 (9), 1753-7.

44.Sachdeva, A.; Chandra, M.; Chandrasekar, J.; Silverman, S. K., Covalent tagging of phosphorylated peptides by phosphate-specific deoxyribozymes. Chembiochem : a European journal of chemical biology 2012, 13 (5), 654-7.

45.Ahrendt, K. A.; Borths, C. J.; MacMillan, D. W. C., New Strategies for Organic Catalysis: The First Highly Enantioselective Organocatalytic Diels-Alder Reaction. Journal of the American Chemical Society 2000, 122 (17), 4243-4244. 
46.Chandra, M.; Silverman, S. K., DNA and RNA can be equally efficient catalysts for carbon-carbon bond formation. Journal of the American Chemical Society 2008, 130 (10), 2936-7.

47.Mohan, U.; Burai, R.; McNaughton, B. R., In vitro evolution of a Friedel-Crafts deoxyribozyme. Organic \& biomolecular chemistry 2013, 11 (14), 2241-4.

48.Li, Y.; Sen, D., A catalytic DNA for porphyrin metallation. Nature structural biology 1996, 3 (9), 7437.

49.Xue, T.; Jiang, S.; Qu, Y.; Su, Q.; Cheng, R.; Dubin, S.; Chiu, C.-Y.; Kaner, R.; Huang, Y.; Duan, X., Graphene-Supported Hemin as a Highly Active Biomimetic Oxidation Catalyst. Angewandte Chemie International Edition 2012, 51 (16), 3822-3825.

50.Travascio, P.; Li, Y.; Sen, D., DNA-enhanced peroxidase activity of a DNA-aptamer-hemin complex. Chemistry \& biology 1998, 5 (9), 505-17.

51.Chinnapen, D. J.; Sen, D., A deoxyribozyme that harnesses light to repair thymine dimers in DNA. Proc Natl Acad Sci U S A 2004, 101 (1), 65-9.

52.Barlev, A.; Sen, D., Catalytic DNAs that harness violet light to repair thymine dimers in a DNA substrate. Journal of the American Chemical Society 2013, 135 (7), 2596-603.

53.Sachdeva, A.; Silverman, S. K., DNA-catalyzed reactivity of a phosphoramidate functional group and formation of an unusual pyrophosphoramidate linkage. Organic \& biomolecular chemistry 2012, 10 (1), $122-5$.

54.Wong, O. Y.; Mulcrone, A. E.; Silverman, S. K., DNA-catalyzed reductive amination. Angewandte Chemie (International ed. in English) 2011, 50 (49), 11679-84.

55.Sheppard, T. L.; Ordoukhanian, P.; Joyce, G. F., A DNA enzyme with N-glycosylase activity. Proceedings of the National Academy of Sciences 2000, 97 (14), 7802-7807.

56. Hobartner, C.; Pradeepkumar, P. I.; Silverman, S. K., Site-selective depurination by a periodatedependent deoxyribozyme. Chemical communications (Cambridge, England) 2007, (22), 2255-7.

57.Seelig, B.; Jaschke, A., A small catalytic RNA motif with Diels-Alderase activity. Chemistry \& biology 1999, 6 (3), 167-76.

58.(a) Stuhlmann, F.; Jaschke, A., Characterization of an RNA active site: interactions between a DielsAlderase ribozyme and its substrates and products. Journal of the American Chemical Society 2002, 124 (13), 3238-44; (b) Serganov, A.; Keiper, S.; Malinina, L.; Tereshko, V.; Skripkin, E.; Hobartner, C.; Polonskaia, A.; Phan, A. T.; Wombacher, R.; Micura, R.; Dauter, Z.; Jaschke, A.; Patel, D. J., Structural basis for Diels-Alder ribozyme-catalyzed carbon-carbon bond formation. Nature structural \& molecular biology 2005, 12 (3), 218-24.

59.Kim, J. E.; Yoon, S.; Mok, H.; Jung, W.; Kim, D. E., Site-specific cleavage of mutant ABL mRNA by DNAzyme is facilitated by peptide nucleic acid binding to RNA substrate. FEBS letters 2012, 586 (21), 3865-9.

60.Singh, N.; Ranjan, A.; Sur, S.; Chandra, R.; Tandon, V., Inhibition of HIV-1 Integrase gene expression by 10-23 DNAzyme. Journal of biosciences 2012, 37 (3), 493-502.

61.Dicke, T.; Pali-Scholl, I.; Kaufmann, A.; Bauer, S.; Renz, H.; Garn, H., Absence of unspecific innate immune cell activation by GATA-3-specific DNAzymes. Nucleic acid therapeutics 2012, 22 (2), 117-26. 62.Li, J.; Wang, N.; Luo, Q.; Wan, L., The 10-23 DNA enzyme generated by a novel expression vector mediate inhibition of taco expression in macrophage. Oligonucleotides 2010, 20 (2), 61-8.

63.Cheglakov, Z.; Weizmann, Y.; Basnar, B.; Willner, I., Diagnosing viruses by the rolling circle amplified synthesis of DNAzymes. Organic \& biomolecular chemistry 2007, 5 (2), 223-5.

64.Zhu, G.; Ye, M.; Donovan, M. J.; Song, E.; Zhao, Z.; Tan, W., Nucleic acid aptamers: an emerging frontier in cancer therapy. Chemical communications (Cambridge, England) 2012, 48 (85), 10472-80.

65.Liu, J.; Cao, Z.; Lu, Y., Functional nucleic acid sensors. Chemical reviews 2009, 109 (5), 1948-98.

66.Lee, J. F.; Hesselberth, J. R.; Meyers, L. A.; Ellington, A. D., Aptamer database. Nucleic acids research 2004, 32 (Database issue), D95-100.

67.Cruz-Toledo, J.; McKeague, M.; Zhang, X.; Giamberardino, A.; McConnell, E.; Francis, T.; DeRosa, M. C.; Dumontier, M., Aptamer base: a collaborative knowledge base to describe aptamers and SELEX experiments. Database 2012, 2012. 
68.Shlyahovsky, B.; Pavlov, V.; Kaganovsky, L.; Willner, I., Biocatalytic Evolution of a Biocatalyst Marker: Towards the Ultrasensitive Detection of Immunocomplexes and DNA Analysis. Angewandte Chemie International Edition 2006, 45 (29), 4815-4819.

69.Li, C. L.; Huang, C. C.; Chen, W. H.; Chiang, C. K.; Chang, H. T., Peroxidase mimicking DNA-gold nanoparticles for fluorescence detection of the lead ions in blood. The Analyst 2012, 137 (22), 5222-8. 70.Zhang, M.; Xu, S.; Minteer, S. D.; Baum, D. A., Investigation of a Deoxyribozyme As a Biofuel Cell Catalyst. Journal of the American Chemical Society 2011, 133 (40), 15890-15893.

71.Weizmann, Y.; Beissenhirtz, M. K.; Cheglakov, Z.; Nowarski, R.; Kotler, M.; Willner, I., A Virus Spotlighted by an Autonomous DNA Machine. Angewandte Chemie International Edition 2006, 45 (44), 7384-7388.

72.Yan, C.; Jiang, C.; Jiang, J.; Yu, R., Simple, Colorimetric Detection of MicroRNA Based on Target Amplification and DNAzyme. Analytical sciences : the international journal of the Japan Society for Analytical Chemistry 2013, 29 (6), 605-10.

73.Wang, F.; Orbach, R.; Willner, I., Detection of metal ions (Cu2+, $\mathrm{Hg} 2+$ ) and cocaine by using ligation DNAzyme machinery. Chemistry (Weinheim an der Bergstrasse, Germany) 2012, 18 (50), 16030-6.

74.Zhang, J.; Gao, Q.; Chen, P.; Chen, J.; Chen, G.; Fu, F., A novel Tb(3+)-promoted G-quadruplex-hemin DNAzyme for the development of label-free visual biosensors. Biosensors \& bioelectronics 2011, 26 (10), 4053-7.

75.Liu, J.; Lu, Y., A colorimetric lead biosensor using DNAzyme-directed assembly of gold nanoparticles. Journal of the American Chemical Society 2003, 125 (22), 6642-3.

76.Mazumdar, D.; Nagraj, N.; Kim, H. K.; Meng, X.; Brown, A. K.; Sun, Q.; Li, W.; Lu, Y., Activity, folding and Z-DNA formation of the 8-17 DNAzyme in the presence of monovalent ions. Journal of the American Chemical Society 2009, 131 (15), 5506-15.

77.Xiao, Y.; Rowe, A. A.; Plaxco, K. W., Electrochemical detection of parts-per-billion lead via an electrode-bound DNAzyme assembly. Journal of the American Chemical Society 2007, 129 (2), 262-3.

78.Baum, D. A.; Silverman, S. K., Deoxyribozyme-catalyzed labeling of RNA. Angewandte Chemie (International ed. in English) 2007, 46 (19), 3502-4.

79.Buttner, L.; Seikowski, J.; Wawrzyniak, K.; Ochmann, A.; Hobartner, C., Synthesis of spin-labeled riboswitch RNAs using convertible nucleosides and DNA-catalyzed RNA ligation. Bioorganic \& medicinal chemistry 2013.

80.Blahna, M. T.; Hata, A., Regulation of miRNA biogenesis as an integrated component of growth factor signaling. Current opinion in cell biology 2013, 25 (2), 233-40.

81.Siomi, H.; Siomi, M. C., On the road to reading the RNA-interference code. Nature 2009, 457 (7228), 396-404.

82.Marchfelder, A.; Fischer, S.; Brendel, J.; Stoll, B.; Maier, L. K.; Jager, D.; Prasse, D.; Plagens, A.; Schmitz, R. A.; Randau, L., Small RNAs for defence and regulation in archaea. Extremophiles : life under extreme conditions 2012, 16 (5), 685-96.

83.Le Rhun, A.; Charpentier, E., Small RNAs in streptococci. RNA biology 2012, 9 (4), 414-26.

84.Zhang, J.; Lau, M. W.; Ferre-D'Amare, A. R., Ribozymes and riboswitches: modulation of RNA function by small molecules. Biochemistry 2010, 49 (43), 9123-31.

85.Collins, J. A.; Irnov, I.; Baker, S.; Winkler, W. C., Mechanism of mRNA destabilization by the glmS ribozyme. Genes \& development 2007, 21 (24), 3356-68.

86.Li, S.; Breaker, R. R., Eukaryotic TPP riboswitch regulation of alternative splicing involving longdistance base pairing. Nucleic acids research 2013, 41 (5), 3022-31.

87.Wachter, A., Riboswitch-mediated control of gene expression in eukaryotes. RNA biology 2010, 7 (1), 67-76.

88.Wahl, M. C.; Will, C. L.; Luhrmann, R., The spliceosome: design principles of a dynamic RNP machine. Cell 2009, 136 (4), 701-18.

89.Michel, F.; Ferat, J. L., Structure and activities of group II introns. Annual review of biochemistry 1995, 64, 435-61.

90.Wang, Y.; Silverman, S. K., A general two-step strategy to synthesize lariat RNAs. RNA (New York, N.Y.) 2006, 12 (2), 313-21. 
91.Zuker, M.; Stiegler, P., Optimal computer folding of large RNA sequences using thermodynamics and auxiliary information. Nucleic acids research 1981, 9 (1), 133-148.

92.Bompfünewerer, A.; Backofen, R.; Bernhart, S.; Hertel, J.; Hofacker, I.; Stadler, P.; Will, S., Variations on RNA folding and alignment: lessons from Benasque. J. Math. Biol. 2008, 56 (1-2), 129-144.

93. Mathews, D. H., Using an RNA secondary structure partition function to determine confidence in base pairs predicted by free energy minimization. RNA (New York, N.Y.) 2004, 10 (8), 1178-90.

94.Nowakowski, J.; Shim, P. J.; Prasad, G. S.; Stout, C. D.; Joyce, G. F., Crystal structure of an 82nucleotide RNA-DNA complex formed by the 10-23 DNA enzyme. Nature structural biology 1999, 6 (2), 151-6.

95.Grigg, J. C.; Chen, Y.; Grundy, F. J.; Henkin, T. M.; Pollack, L.; Ke, A., T box RNA decodes both the information content and geometry of tRNA to affect gene expression. Proceedings of the National Academy of Sciences 2013.

96.Kim, H.-K.; Rasnik, I.; Liu, J.; Ha, T.; Lu, Y., Dissecting metal ion-dependent folding and catalysis of a single DNAzyme. Nat Chem Biol 2007, 3 (12), 763-768.

97.Steitz, T. A.; Steitz, J. A., A general two-metal-ion mechanism for catalytic RNA. Proc Natl Acad Sci U SA 1993, 90 (14), 6498-502.

98.Lu, Y., New Transition-Metal-Dependent DNAzymes as Efficient Endonucleases and as Selective Metal Biosensors. Chemistry - A European Journal 2002, 8 (20), 4588-4596.

99.Romani, A., Regulation of magnesium homeostasis and transport in mammalian cells. Archives of biochemistry and biophysics 2007, 458 (1), 90-102.

100. Grubbs, R. D., Intracellular magnesium and magnesium buffering. Biometals : an international journal on the role of metal ions in biology, biochemistry, and medicine 2002, 15 (3), 251-9.

101. Laing, L. G.; Gluick, T. C.; Draper, D. E., Stabilization of RNA structure by Mg ions. Specific and non-specific effects. Journal of molecular biology 1994, 237 (5), 577-87.

102. Soper, A. K.; Weckström, K., lon solvation and water structure in potassium halide aqueous solutions. Biophysical chemistry 2006, 124 (3), 180-191.

103. Anderson, C. F.; Record, M. T., Jr., Salt-nucleic acid interactions. Annual review of physical chemistry 1995, 46, 657-700.

104. Misra, V. K.; Draper, D. E., On the role of magnesium ions in RNA stability. Biopolymers 1998, 48 (2-3), 113-35.

105. Geyer, C. R.; Sen, D., Evidence for the metal-cofactor independence of an RNA phosphodiestercleaving DNA enzyme. Chemistry \& biology 1997, 4 (8), 579-93.

106. Sen, D.; Gilbert, W., A sodium-potassium switch in the formation of four-stranded G4-DNA. Nature 1990, 344 (6265), 410-414.

107. Wojtuszewski Poulin, K.; Smirnov, A. V.; Hawkins, M. E.; Balis, F. M.; Knutson, J. R., Conformational heterogeneity and quasi-static self-quenching in DNA containing a fluorescent guanine analogue, 3MI or 6MI. Biochemistry 2009, 48 (37), 8861-8.

108. Murray, J. B.; Seyhan, A. A.; Walter, N. G.; Burke, J. M.; Scott, W. G., The hammerhead, hairpin and VS ribozymes are catalytically proficient in monovalent cations alone. Chemistry \& biology 1998, 5 (10), 587-595.

109. Young, K. J.; Gill, F.; Grasby, J. A., Metal ions play a passive role in the hairpin ribozyme catalysed reaction. Nucleic acids research 1997, 25 (19), 3760-6.

110. Horton, T. E.; DeRose, V. J., Cobalt Hexammine Inhibition of the Hammerhead Ribozymet. Biochemistry 2000, 39 (37), 11408-11416.

111. Geyer, C. R.; Sen, D., Lanthanide probes for a phosphodiester-cleaving, lead-dependent, DNAzyme. Journal of molecular biology 1998, 275 (3), 483-9.

112. Shannon, R. D., Revised Effective Ionic Radii and Systematic Studies of Interatomic Distances in Halides and Chalcogenides. Acta Crystallographica. 1976, A32, 751-767.

113. Feig, A. L.; Panek, M.; Horrocks, W. D., Jr.; Uhlenbeck, O. C., Probing the binding of Tb(III) and $\mathrm{Eu}(\mathrm{III})$ to the hammerhead ribozyme using luminescence spectroscopy. Chemistry \& biology 1999, 6 (11), 801-10. 
114. Auffinger, P.; Grover, N.; Westhof, E., Metal ion binding to RNA. Metal ions in life sciences 2011, 9, 1-35.

115. Morrow, J. R.; Andolina, C. M., Spectroscopic investigations of lanthanide ion binding to nucleic acids. Metal ions in life sciences 2012, 10, 171-99.

116. Feng, X.; Ling, X. L.; Liu, L.; Song, H. L.; Wang, L. Y.; Ng, S. W.; Su, B. Y., A series of 3D lanthanide frameworks constructed from aromatic multi-carboxylate ligand: Structural diversity, luminescence and magnetic properties. Dalton transactions (Cambridge, England : 2003) 2013, 42 (28), 10292-303.

117. Petoud, S.; Cohen, S. M.; Bunzli, J. C.; Raymond, K. N., Stable lanthanide luminescence agents highly emissive in aqueous solution: multidentate 2-hydroxyisophthalamide complexes of $\mathrm{Sm}(3+)$, $\mathrm{Eu}(3+), \mathrm{Tb}(3+), \mathrm{Dy}(3+)$. Journal of the American Chemical Society 2003, 125 (44), 13324-5.

118. Boulon, M. E.; Cucinotta, G.; Luzon, J.; Degl'Innocenti, C.; Perfetti, M.; Bernot, K.; Calvez, G.; Caneschi, A.; Sessoli, R., Magnetic anisotropy and spin-parity effect along the series of lanthanide complexes with DOTA. Angewandte Chemie (International ed. in English) 2013, 52 (1), 350-4.

119. Penas, C.; Pazos, E.; Mascareñas, J. L.; Vázquez, M. E., A Folding-Based Approach for the Luminescent Detection of a Short RNA Hairpin. Journal of the American Chemical Society 2013, 135 (10), 3812-3814.

120. Yegorova, A. V.; Scripinets, Y. V.; Duerkop, A.; Karasyov, A. A.; Antonovich, V. P.; Wolfbeis, O. S., Sensitive luminescent determination of DNA using the terbium(III)-difloxacin complex. Analytica chimica acta 2007, 584 (2), 260-7.

121. Yuan, F.; Griffin, L.; Phelps, L.; Buschmann, V.; Weston, K.; Greenbaum, N. L., Use of a novel Forster resonance energy transfer method to identify locations of site-bound metal ions in the U2-U6 snRNA complex. Nucleic acids research 2007, 35 (9), 2833-45.

122. Nagesh, N.; Bhargava, P.; Chatterji, D., Terbium(III)-induced fluorescence of four-stranded G4DNA. Biopolymers 1992, 32 (10), 1421-4.

123. Worlinsky, J. L.; Basu, S., Detection of quadruplex DNA by luminescence enhancement of lanthanide ions and energy transfer from lanthanide chelates. The journal of physical chemistry. $B$ 2009, $113(4), 865-8$.

124. Galezowska, E.; Gluszynska, A.; Juskowiak, B., Luminescence study of G-quadruplex formation in the presence of Tb3+ ion. Journal of inorganic biochemistry 2007, 101 (4), 678-85.

125. Draper, D. E., On the coordination properties of Eu3+ bound to tRNA. Biophysical chemistry 1985, 21 (2), 91-101.

126. Walter, N. G.; Yang, N.; Burke, J. M., Probing non-selective cation binding in the hairpin ribozyme with $\mathrm{Tb}(\mathrm{III})$. Journal of molecular biology 2000, 298 (3), 539-55.

127. Heyduk, T., Measuring protein conformational changes by FRET/LRET. Current opinion in biotechnology 2002, 13 (4), 292-296.

128. Hargittai, M. R.; Musier-Forsyth, K., Use of terbium as a probe of tRNA tertiary structure and folding. RNA (New York, N.Y.) 2000, 6 (11), 1672-80.

129. Harris, D. A.; Tinsley, R. A.; Walter, N. G., Terbium-mediated footprinting probes a catalytic conformational switch in the antigenomic hepatitis delta virus ribozyme. Journal of molecular biology 2004, 341 (2), 389-403.

130. Camargo, M. A.; Neves, A.; Bortoluzzi, A. J.; Szpoganicz, B.; Fischer, F. L.; Terenzi, H.; Serra, O. A.; Santos, V. G.; Vaz, B. G.; Eberlin, M. N., Efficient phosphodiester hydrolysis by luminescent terbium(III) and europium(III) complexes. Inorganic chemistry 2010, 49 (13), 6013-25.

131. Hall, J.; Husken, D.; Haner, R., Towards artificial ribonucleases: the sequence-specific cleavage of RNA in a duplex. Nucleic acids research 1996, 24 (18), 3522-6.

132. Nakamatsu, Y.; Warashina, M.; Kuwabara, T.; Tanaka, Y.; Shibata, A.; Yoshinari, K.; Taira, K., Action of metal ions directly involved in the cleavage reaction of hammerhead ribozymes. Nucleic acids symposium series 1999, (42), 283-4.

133. Ohmichi, T.; Sugimoto, N., Role of $\mathrm{Nd} 3+$ and $\mathrm{Pb} 2+$ on the RNA cleavage reaction by a small ribozyme. Biochemistry 1997, 36 (12), 3514-21. 
134. Lott, W. B.; Pontius, B. W.; von Hippel, P. H., A two-metal ion mechanism operates in the hammerhead ribozyme-mediated cleavage of an RNA substrate. Proc Natl Acad Sci U S A 1998, 95 (2), 542-7.

135. Kim, H. K.; Li, J.; Nagraj, N.; Lu, Y., Probing metal binding in the 8-17 DNAzyme by TbllI luminescence spectroscopy. Chemistry (Weinheim an der Bergstrasse, Germany) 2008, 14 (28), 8696703.

136. Gaur, R. K.; Paliwal, S.; Sharma, P.; Gupta, K. C., A simple and sensitive spectrophotometric method for the quantitative determination of solid supported amino groups. Journal of biochemical and biophysical methods 1989, 18 (4), 323-9.

137. Pitsch, S.; Weiss, P. A., Preparation of 2'-O-[(Triisopropylsilyl)oxy]methyl-protected ribonucleosides. Current protocols in nucleic acid chemistry / edited by Serge L. Beaucage ... [et al.] 2002, Chapter 2, Unit 29.

138. Höbartner, C.; Kreutz, C.; Flecker, E.; Ottenschläger, E.; Pils, W.; Grubmayr, K.; Micura, R., The Synthesis of 2'-O-[(Triisopropylsilyl)oxy] methyl (TOM) Phosphoramidites of Methylated Ribonucleosides ( $\mathrm{m} 1 \mathrm{G}, \mathrm{m} 2 \mathrm{G}, \mathrm{m} 22 \mathrm{G}, \mathrm{m} 1 \mathrm{I}, \mathrm{m} 3 \mathrm{U}, \mathrm{m} 4 \mathrm{C}, \mathrm{m} 6 \mathrm{~A}, \mathrm{~m} 62 \mathrm{~A}$ ) for Use in Automated RNA Solid-Phase Synthesis. Monatshefte für Chemie 2003, 134 (6), 851-873.

139. Wachowius, F.; Javadi-Zarnaghi, F.; Höbartner, C., Combinatorial mutation interference analysis reveals functional nucleotides required for DNA catalysis. Angew. Chem. Int. Ed. 2010, 49 (45), 8504-8. 140. Wachowius, F.; Höbartner, C., Probing essential nucleobase functional groups in aptamers and deoxyribozymes by nucleotide analogue interference mapping of DNA. Journal of the American Chemical Society 2011, 133 (38), 14888-91.

141. Maxam, A. M.; Gilbert, W., A new method for sequencing DNA. Proc Natl Acad Sci U S A 1977, 74 (2), 560-4.

142. Waldsich, C.; Schroeder, R., In Vivo Determination of RNA Structure by Dimethylsulfate. In Handbook of RNA Biochemistry, Wiley-VCH Verlag GmbH: 2008; pp 229-237.

143. Gilbert, W., DNA sequencing and gene structure. Science (New York, N.Y.) 1981, 214 (4527), 1305-12.

144. Ziehler, W. A.; Engelke, D. R., Probing RNA Structure with Chemical Reagents and Enzymes. In Current Protocols in Nucleic Acid Chemistry, John Wiley \& Sons, Inc.: 2001.

145. Cardew, A. S.; Fox, K. R., DNase I footprinting. Methods in molecular biology (Clifton, N.J.) 2010, 613, 153-72.

146. Forconi, M.; Herschlag, D., Use of Phosphorothioates to Identify Sites of Metal-Ion Binding in RNA. In Methods in Enzymology, Daniel, H., Ed. Academic Press: 2009; Vol. Volume 468, pp 311-333.

147. Piccirilli, J. A.; Vyle, J. S.; Caruthers, M. H.; Cech, T. R., Metal ion catalysis in the Tetrahymena ribozyme reaction. Nature 1993, 361 (6407), 85-8.

148. Feig, A. L.; Scott, W. G.; Uhlenbeck, O. C., Inhibition of the hammerhead ribozyme cleavage reaction by site-specific binding of Tb. Science (New York, N.Y.) 1998, 279 (5347), 81-4.

149. Wang, Y.; Silverman, S. K., Characterization of deoxyribozymes that synthesize branched RNA. Biochemistry 2003, 42 (51), 15252-63.

150. (a) Piccirilli, J. A.; Vyle, J. S.; Caruthers, M. H.; Cech, T. R., Metal ion catalysis in the Tetrahymena ribozyme reaction. Nature 1993, 361 (6407), 85-88; (b) Forconi, M.; Lee, J.; Lee, J. K.; Piccirilli, J. A.; Herschlag, D., Functional Identification of Ligands for a Catalytic Metal Ion in Group I Intronst. Biochemistry 2008, 47 (26), 6883-6894.

151. Bardin, C.; Leroy, J. L., The formation pathway of tetramolecular G-quadruplexes. Nucleic acids research 2008, $36(2), 477-88$.

152. Xu, H.; Zhang, H.; Qu, X., Interactions of the human telomeric DNA with terbium-amino acid complexes. Journal of inorganic biochemistry 2006, 100 (10), 1646-1652.

153. Vorlickova, M.; Kejnovska, I.; Bednarova, K.; Renciuk, D.; Kypr, J., Circular dichroism spectroscopy of DNA: from duplexes to quadruplexes. Chirality 2012, 24 (9), 691-8.

154. Jangir, D. K.; Charak, S.; Mehrotra, R.; Kundu, S., FTIR and circular dichroism spectroscopic study of interaction of 5-fluorouracil with DNA. Journal of Photochemistry and Photobiology B: Biology 2011, 105 (2), 143-148. 
155. Charles, I.; Davis, E.; Arya, D. P., Efficient stabilization of phosphodiester (PO), phosphorothioate (PS), and 2'-O-methoxy (2'-OMe) DNA.RNA hybrid duplexes by amino sugars. Biochemistry 2012, 51 (27), 5496-505.

156. Blume, S. W.; Guarcello, V.; Zacharias, W.; Miller, D. M., Divalent transition metal cations counteract potassium-induced quadruplex assembly of oligo(dG) sequences. Nucleic acids research 1997, 25 (3), 617-25.

157. Pearson, A. M.; Rich, A.; Krieger, M., Polynucleotide binding to macrophage scavenger receptors depends on the formation of base-quartet-stabilized four-stranded helices. The Journal of biological chemistry 1993, 268 (5), 3546-54.

158. Yamashita, T.; Uno, T.; Ishikawa, Y., Stabilization of guanine quadruplex DNA by the binding of porphyrins with cationic side arms. Bioorganic \& medicinal chemistry 2005, 13 (7), 2423-2430.

159. Izbicka, E.; Wheelhouse, R. T.; Raymond, E.; Davidson, K. K.; Lawrence, R. A.; Sun, D.; Windle, B. E.; Hurley, L. H.; Von Hoff, D. D., Effects of cationic porphyrins as G-quadruplex interactive agents in human tumor cells. Cancer research 1999, 59 (3), 639-44.

160. Wei, C.; Jia, G.; Yuan, J.; Feng, Z.; Li, C., A spectroscopic study on the interactions of porphyrin with G-quadruplex DNAs. Biochemistry 2006, 45 (21), 6681-91.

161. Bochman, M. L.; Paeschke, K.; Zakian, V. A., DNA secondary structures: stability and function of G-quadruplex structures. Nature reviews. Genetics 2012, 13 (11), 770-80.

162. Kosman, J.; Juskowiak, B., Peroxidase-mimicking DNAzymes for biosensing applications: a review. Analytica chimica acta 2011, 707 (1-2), 7-17.

163. Arthanari, H.; Basu, S.; Kawano, T. L.; Bolton, P. H., Fluorescent dyes specific for quadruplex DNA. Nucleic acids research 1998, 26 (16), 3724-8.

164. Xu, H.; Zhang, H.; Qu, X., Interactions of the human telomeric DNA with terbium-amino acid complexes. Journal of inorganic biochemistry 2006, 100 (10), 1646-52.

165. Krafft, C.; Benevides, J. M.; Thomas, G. J., Jr., Secondary structure polymorphism in Oxytricha nova telomeric DNA. Nucleic acids research 2002, 30 (18), 3981-91.

166. Pagba, C. V.; Lane, S. M.; Wachsmann-Hogiu, S., Conformational changes in quadruplex oligonucleotide structures probed by Raman spectroscopy. Biomedical optics express 2010, 2 (2), 20717.

167. Miura, T.; Thomas, G. J., Jr., Structural polymorphism of telomere DNA: interquadruplex and duplex-quadruplex conversions probed by Raman spectroscopy. Biochemistry 1994, 33 (25), 7848-56.

168. Zhang, N.; Zhang, S.; Szostak, J. W., Activated ribonucleotides undergo a sugar pucker switch upon binding to a single-stranded RNA template. Journal of the American Chemical Society 2012, 134 (8), 3691-4.

169. Berg JM, T. J., Stryer L. , DNA Can Assume a Variety of Structural Forms. In Biochemistry, 5th edition ed.; New York: W H Freeman: 2002.

170. Soukup, J. K.; Minakawa, N.; Matsuda, A.; Strobel, S. A., Identification of A-minor tertiary interactions within a bacterial group I intron active site by 3-deazaadenosine interference mapping. Biochemistry 2002, 41 (33), 10426-38.

171. He, H.-Z.; Chan, D. S.-H.; Leung, C.-H.; Ma, D.-L., G-quadruplexes for luminescent sensing and logic gates. Nucleic acids research 2013.

172. Miyoshi, D.; Nakao, A.; Sugimoto, N., Structural transition from antiparallel to parallel Gquadruplex of d(G4T4G4) induced by Ca2+. Nucleic acids research 2003, 31 (4), 1156-1163.

173. Vuong, S.; Stefan, L.; Lejault, P.; Rousselin, Y.; Denat, F.; Monchaud, D., Identifying three-way DNA junction-specific small-molecules. Biochimie 2012, 94 (2), 442-450.

174. Malinovskii, V. L.; Nussbaumer, A. L.; Haner, R., Oligopyrenotides: chiral nanoscale templates for chromophore assembly. Angewandte Chemie (International ed. in English) 2012, 51 (20), 4905-8.

175. Vasudevan, S. S.; Sundaralingam, M., The occurence of the syn-C3' endo conformation and the distorted backbone conformations for $\mathrm{C}^{\prime} \mathrm{C}^{-} 5^{\prime}$ and $\mathrm{P}-\mathrm{O} 5^{\prime}$ in oligo and polynucleotides. Journal of biomolecular structure \& dynamics 2001, 18 (6), 824-31.

176. Carriero, S.; Damha, M. J., Solid-phase synthesis of branched oligonucleotides. Current protocols in nucleic acid chemistry / edited by Serge L. Beaucage ... [et al.] 2002, Chapter 4, Unit 414. 
177. Nakashima, Y.; Abe, H.; Abe, N.; Aikawa, K.; Ito, Y., Branched RNA nanostructures for RNA interference. Chemical communications (Cambridge, England) 2011, 47 (29), 8367-9.

178. Panja, S.; Woodson, S. A., Hfq proximity and orientation controls RNA annealing. Nucleic acids research 2012, 40 (17), 8690-7.

179. Höbartner, C.; Silverman, S. K., Engineering a selective small-molecule substrate binding site into a deoxyribozyme. Angew. Chem. Int. Ed. 2007, 46 (39), 7420-4.

180. Fuchs, R. T.; Grundy, F. J.; Henkin, T. M., The S(MK) box is a new SAM-binding RNA for translational regulation of SAM synthetase. Nature structural \& molecular biology 2006, 13 (3), 226-33. 181. Wilson, R. C.; Smith, A. M.; Fuchs, R. T.; Kleckner, I. R.; Henkin, T. M.; Foster, M. P., Tuning riboswitch regulation through conformational selection. Journal of molecular biology 2011, 405 (4), 926-38.

182. Lu, C.; Smith, A. M.; Fuchs, R. T.; Ding, F.; Rajashankar, K.; Henkin, T. M.; Ke, A., Crystal structures of the SAM-III/S(MK) riboswitch reveal the SAM-dependent translation inhibition mechanism. Nature structural \& molecular biology 2008, 15 (10), 1076-83.

183. Santoro, S. W.; Joyce, G. F., Mechanism and utility of an RNA-cleaving DNA enzyme. Biochemistry 1998, 37 (38), 13330-42.

184. Wahl, M. C.; Will, C. L.; Lührmann, R., The Spliceosome: Design Principles of a Dynamic RNP Machine. Cell 2009, 136 (4), 701-718.

185. Fourmann, J. B.; Schmitzova, J.; Christian, H.; Urlaub, H.; Ficner, R.; Boon, K. L.; Fabrizio, P.; Luhrmann, R., Dissection of the factor requirements for spliceosome disassembly and the elucidation of its dissociation products using a purified splicing system. Genes \& development 2013, 27 (4), 413-28. 186. Liu, Y. C.; Chen, H. C.; Wu, N. Y.; Cheng, S. C., A novel splicing factor, Yju2, is associated with NTC and acts after Prp2 in promoting the first catalytic reaction of pre-mRNA splicing. Molecular and cellular biology 2007, 27 (15), 5403-13.

187. Mozaffari-Jovin, S.; Wandersleben, T.; Santos, K. F.; Will, C. L.; Luhrmann, R.; Wahl, M. C., Inhibition of RNA Helicase Brr2 by the C-Terminal Tail of the Spliceosomal Protein Prp8. Science (New York, N.Y.) 2013.

188. Pena, V.; Rozov, A.; Fabrizio, P.; Luhrmann, R.; Wahl, M. C., Structure and function of an RNase H domain at the heart of the spliceosome. The EMBO journal 2008, 27 (21), 2929-40.

189. Chan, S. P.; Cheng, S. C., The Prp19-associated complex is required for specifying interactions of U5 and U6 with pre-mRNA during spliceosome activation. The Journal of biological chemistry 2005, 280 (35), 31190-9.

190. McGrail, J. C.; Krause, A.; O'Keefe, R. T., The RNA binding protein Cwc2 interacts directly with the U6 snRNA to link the nineteen complex to the spliceosome during pre-mRNA splicing. Nucleic acids research 2009, 37 (13), 4205-17.

191. Rasche, N.; Dybkov, O.; Schmitzova, J.; Akyildiz, B.; Fabrizio, P.; Luhrmann, R., Cwc2 and its human homologue RBM22 promote an active conformation of the spliceosome catalytic centre. The EMBO journal 2012, 31 (6), 1591-604.

192. Schmitzova, J.; Rasche, N.; Dybkov, O.; Kramer, K.; Fabrizio, P.; Urlaub, H.; Luhrmann, R.; Pena, V., Crystal structure of CwC2 reveals a novel architecture of a multipartite RNA-binding protein. The EMBO journal 2012, 31 (9), 2222-34.

193. Gao, P.; Ascano, M.; Wu, Y.; Barchet, W.; Gaffney, B. L.; Zillinger, T.; Serganov, A. A.; Liu, Y.; Jones, R. A.; Hartmann, G.; Tuschl, T.; Patel, D. J., Cyclic $\left[G\left(2^{\prime}, 5^{\prime}\right) p A\left(3^{\prime}, 5^{\prime}\right) p\right]$ is the metazoan second messenger produced by DNA-activated cyclic GMP-AMP synthase. Cell 2013, 153 (5), 1094-107.

194. Dey, B.; Thukral, S.; Krishnan, S.; Chakrobarty, M.; Gupta, S.; Manghani, C.; Rani, V., DNA-protein interactions: methods for detection and analysis. Molecular and cellular biochemistry 2012, 365 (1-2), 279-99.

195. Holden, N. S.; Tacon, C. E., Principles and problems of the electrophoretic mobility shift assay. Journal of Pharmacological and Toxicological Methods 2011, 63 (1), 7-14.

196. Anna, Z.; Michael, Y. P.; Annika, I. N.; Måns, E.; Dan, I. A., Error-prone initiation factor 2 mutations reduce the fitness cost of antibiotic resistance. Molecular Microbiology 2010, 75 (5), 12991313. 
197. Milon, P.; Carotti, M.; Konevega, A. L.; Wintermeyer, W.; Rodnina, M. V.; Gualerzi, C. O., The ribosome-bound initiation factor 2 recruits initiator tRNA to the $30 \mathrm{~S}$ initiation complex. EMBO reports 2010, 11 (4), 312-6.

198. Milon, P.; Maracci, C.; Filonava, L.; Gualerzi, C. O.; Rodnina, M. V., Real-time assembly landscape of bacterial $30 \mathrm{~S}$ translation initiation complex. Nature structural \& molecular biology 2012, 19 (6), 60915.

199. Rodnina, M. V., Long-range signalling in activation of the translational GTPase EF-Tu. The EMBO journal 2009, 28 (6), 619-20.

200. Tanner, D. R.; Hedrick, E. G.; Hill, W. E., Real-time evidence for EF-G-induced dynamics of helix 44 in $16 \mathrm{~S}$ rRNA. Journal of molecular biology 2012, 422 (1), 45-57.

201. Chen, C.; Zhang, H.; Broitman, S. L.; Reiche, M.; Farrell, I.; Cooperman, B. S.; Goldman, Y. E., Dynamics of translation by single ribosomes through mRNA secondary structures. Nature structural \& molecular biology 2013.

202. Thomas, A. S., A structural understanding of the dynamic ribosome machine. Nature Reviews Molecular Cell Biology 2008, 9 (3), 242-253.

203. Brierley, I.; Meredith, M. R.; Bloys, A. J.; Hagervall, T. G., Expression of a coronavirus ribosomal frameshift signal in Escherichia coli: influence of tRNA anticodon modification on frameshifting. Journal of molecular biology 1997, 270 (3), 360-73.

204. Herr, A. J.; Wills, N. M.; Nelson, C. C.; Gesteland, R. F.; Atkins, J. F., Drop-off during ribosome hopping. Journal of molecular biology 2001, 311 (3), 445-52.

205. Yang, Z.; Shipman, L.; Zhang, M.; Anton, B. P.; Roberts, R. J.; Cheng, X., Structural characterization and comparative phylogenetic analysis of Escherichia coli HemK, a protein (N5)glutamine methyltransferase. Journal of molecular biology 2004, 340 (4), 695-706.

206. Burakovsky, D. E.; Prokhorova, I. V.; Sergiev, P. V.; Milon, P.; Sergeeva, O. V.; Bogdanov, A. A.; Rodnina, M. V.; Dontsova, O. A., Impact of methylations of m2G966/m5C967 in 16S rRNA on bacterial fitness and translation initiation. Nucleic acids research 2012, 40 (16), 7885-95.

207. Doudna, J. A.; Cech, T. R., The chemical repertoire of natural ribozymes. Nature 2002, 418 (6894), 222-8.

208. Nagy, V.; Pirakitikulr, N.; Zhou, K. I.; Chillon, I.; Luo, J.; Pyle, A. M., Predicted group II intron lineages $\mathrm{E}$ and $\mathrm{F}$ comprise catalytically active ribozymes. RNA (New York, N.Y.) 2013, 19 (9), 1266-78.

209. Zimmerly, S.; Moran, J. V.; Perlman, P. S.; Lambowitz, A. M., Group II intron reverse transcriptase in yeast mitochondria. Stabilization and regulation of reverse transcriptase activity by the intron RNA. Journal of molecular biology 1999, 289 (3), 473-90.

210. Samanta, B.; Höbartner, C., Combinatorial Nucleoside-Deletion-Scanning Mutagenesis of Functional DNA. Angewandte Chemie International Edition 2013, 52 (10), 2995-2999.

211. Dalgarno, P. A.; Bordello, J.; Morris, R.; St-Pierre, P.; Dubé, A.; Samuel, I. D. W.; Lafontaine, D. A.; Penedo, J. C., Single-molecule chemical denaturation of riboswitches. Nucleic acids research 2013, 41 (7), 4253-4265.

212. Andrus, A.; Kuimelis, R. G., Polyacrylamide gel electrophoresis (PAGE) of synthetic nucleic acids. Current protocols in nucleic acid chemistry / edited by Serge L. Beaucage ... [et al.] 2001, Chapter 10, Unit 104. 